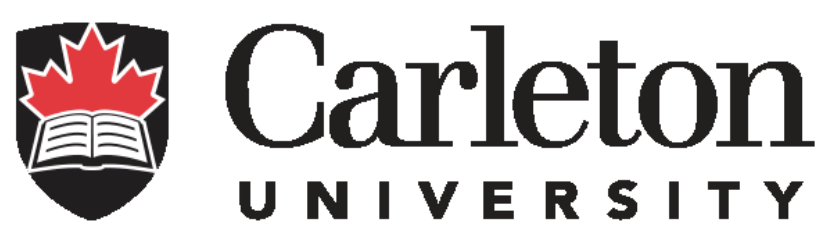

\title{
Functional Applications of Systems Biology Tools: Identification of Novel DNA Repair Factors and Peptide Design
}

\section{Daniel Burnside}

A thesis submitted to the Faculty of Graduate and Post-Doctoral Affairs in partial fulfillment of the requirements for the degree of

\section{Doctor of Philosophy}

in

\section{Biology}

\author{
Department of Biology \\ Ottawa-Carleton Institute of Biology \\ Carleton University \\ Ottawa, Ontario
}

(C) 2019, Daniel Burnside 


\section{Abstract}

The highly annotated budding yeast Saccharomyces cerevisiae has emerged as the primary model for systems biology, the study of how individual cellular components function within the context of a dynamic cellular system. Several genome/proteomescale tools developed using the $S$. cerevisiae model have produced extensive information on gene function and interaction networks that is stored in publicly accessible databases. Bioinformatic tools can exploit these databases to infer novel biological activity but these predictions must be tested in functioning cellular systems to assess the effectiveness of any method. The work herein uses systems-based computational tools to make predictions on novel protein/gene function that are tested using yeast functional genomic approaches. This thesis describes the development and validation of a new tool to design synthetic binding proteins that bind to and inhibit targeted yeast proteins Psk1 and Pin4 as well as the identification and functional analysis of three yeast DNA repair genes, PSK1, ARP6, and DEF1. The in-silico protein synthesizer, InSiPS successfully engineered two synthetic proteins known as anti-Psk1 and anti-Pin4. This demonstrated the ability of our approach to translate from computational prediction, to a specific biological interaction and importantly, a functionally significant phenotype. Chemical-genetic interaction analysis showed that cells expressing $\alpha$-Psk1 and $\alpha$-Pin4 phenocopy $\Delta p s k 1$ and $\Delta p i n 4$ mutants and yeast-twohybrid confirmed binary interactions in vivo while in vitro assays verify that binding is 
occurring at predicted loci. Further analysis of the anti-Psk1/Psk1 interaction motif showed strong, specific binding. Psk1 was inferred to participate in yeast nonhomologous end-joining (NHEJ) repair of double-strand breaks (DSB), an essential DNA repair pathway. Our functional genetic analysis showed that PSK1 is an important novel NHEJ gene that contributes to repair fidelity while appearing to function through RAD27 activity. We also report that ARP6, affects NHEJ through the RSC chromatin remodeling complex. Lastly, we identify new properties of the Def1 DNA repair protein in yeast NHEJ and a physical and genetic interaction between Yku80 and Def1. Together, these findings demonstrate the ability to predict novel gene/protein function using computational tools and expand our understanding of eukaryotic DSB repair. 


\section{Dedication}

This work is dedicated to my father Graham Burnside who never stopped

teaching me, driving me and supporting me. Your incredible selflessness and patience cannot be matched. Thank you 


\section{Acknowledgements}

I would like to genuinely thank my supervisor Dr. Ashkan Golshani. You first got me interested in research and have believed in me since I began in your lab. Thank you for all you have taught me and for demanding the best out of me. Thank you for giving me so many opportunities and responsibilities and for being there every step of the way. I would also like to thank Dr. Myron Smith and Dr. Nicholas Corradi for invaluable suggestions, mentoring and support throughout my PhD work.

To my incredible wife Samantha. Thank you for supporting my dreams and for allowing me to pursue this incredible opportunity. I appreciate all the long hard hours you have put in to support our family while I completed my education. Thank you for being my best friend and biggest supporter.

I have also greatly benefitted from the guidance offered by Dr. Bill Willmore, Dr. Alex Wong, Dr. Kyle Biggar, Dr. Shelley Hepworth, Dr. James Green, Dr. Frank Dehne, and Dr. Sylvain Pitre. Dr. Andrew Schoenrock has been a longtime collaborator and I thank you for the opportunity to work together. Thank you to Dr. Jeff Dawson and Dr. Ashkan Golshani for guiding my teaching philosophy. I have also benefitted from caring members of the department including Suzanne Paterson and Sarah-Anne Szabototh who have been incredibly helpful and supportive as well as Ed Bruggink, Laura Thomas and Ruth Hill-Lapense. 
I would like to thank my incredible parents. My family has always encouraged me in anything I chose to pursue. Thank you for supporting me, for challenging me, and most importantly, for putting up with me. Mom you have been a rock. You have never put yourself first and I hope that one day I can begin to make up for all the things you have done for me. Laura, thank you for always being there and for being supportive and interested in my work.

I have had many great colleagues and friends over the years here but I really do want to specifically acknowledge three people who have impacted me significantly. Dr. Mohsen Hooshyar, thank you for being such a good friend, for teaching me the ropes, for always looking out for me, and for being so brutally honest and always saying exactly what needed to be said. Dr. Bahram Samanfar, you have coached me all the way from my days as the new idiot in the lab until I became the old idiot in the lab. Thank you for finding every opportunity to teach me things, to offer help and support and to be a caring and generous friend. To Dr. Houman Moteshareie, thank for being there every step of the journey. We have been able to lean on each other and have each other's backs this whole time from the really bad days to the really good days. Who knew how excited one little yellow test tube can make a person?

Dr. Katayoun Omidi, Maryam Hajikarimloo, and Taylor Potter, you have been such good friends and colleagues. Thank you for your friendship and for all help you have given me in the lab these past years. To Eshan, Urvi, Anna, Brittney, Kaleigh, 
Narges, Sarah and all of the other members of our last past and present. Thank you for all of your hard work, for your friendship, and for your patience.

To my brother and sister, you are just OK. To my incredible family and network of friends, I am so thankful for all of you. My supportive Grandparents, siblings, aunts, uncles, cousins, extended family and fantastic friends have all helped to get me to this point and never doubted me....at least not to my face.

I would like to give thanks to God for bringing me to this point. This journey would have happened without the grace of God and I am forever thankful.

"I waited patiently for the Lord. He inclined me and heard my cry. He drew me up from the desolate pit, out of the miry clay, and set my feet upon a rock"

Psalms 40: 1-3 


\section{Statement of Contribution}

\section{Chapter 2: In silico Engineering of Synthetic Binding Proteins from Random Amino Acid Sequences}

I participated in this collaborative project from the earliest stages and throughout the design of the InSiPS tool. Principally, I designed, oversaw and performed the majority of wet-lab validation experiments and led manuscript preparation. A. Schoenrock was responsible for development of the code and computational analyses. K. Biggar and $\mathrm{H}$. Moteshareie contributed to wet-lab work.

\section{Chapter 3: Pas Kinase Psk1 Affects Non-homologous End Joining Fidelity}

I completed experiments indicating a role for Psk1 in non-homologous end joining, screened for and analyzed genetic interactions, performed fidelity experiments, and identified a functional relationship with Rad27. The PAS kinase complex was originally identified by M. Hooshyar as a potential NHEJ repair factor. This manuscript was written by me. Undergraduate students U. Bhjoo and E. Silva performed some laboratory experiments.

\section{Chapter 4: Actin-Related Protein 6 Influences Chromatin Remodeling Following Double-Strand Breaks}

This project was completed in conjunction with M. Hooshyar who initiated the project and designed the original premise. A. Jesso and I performed plasmid repair assays and SGA analysis. Interpretation of the resultant data and hypothesis development was performed by myself and M. Hooshyar. The manuscript was written jointly. 


\section{Chapter 5: RNAPII Degradation Factor Def1 influences NHEJ}

Yeast Def1 identification, project conceptualization and experimental design was

performed by me. cell cycle assays, JKM analysis, compensation analysis and cloning

were also performed by me. T. Potter completed the homologous recombination assay,

made JKM knockouts, and contributed to data analysis. Probing of SPOT arrays was

performed in conjunction with M. Hoekstra and K. Biggar. 


\section{Table of Contents}

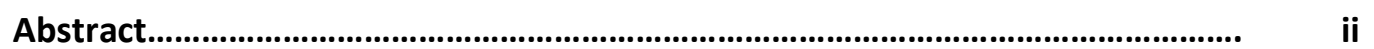

Dedication............................................................................................... iv

Acknowledgements............................................................................... v

Statement of Contribution....................................................................... viii

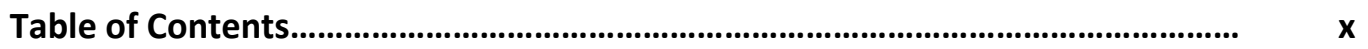

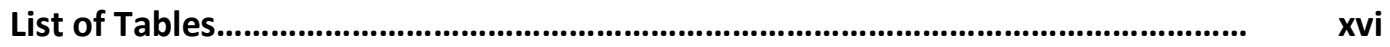

List of Figures......................................................................................... xvii

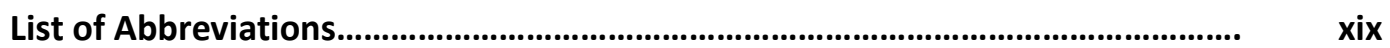

1. Introduction................................................................................... 1

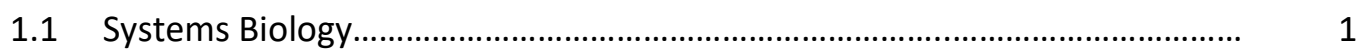

1.1.1 Functional Analysis in Systems Biology........................................... 1

1.1.2 Functional genomics................................................................ 3

1.1.3 Large scale interaction analyses in yeast....................................... 5

1.1.4 Genetic Interactions........................................................... 7

1.1.5 Protein-protein interactions................................................... 10

1.2 DNA Damage and Repair................................................................... 14 
1.2.1 Double-strand breaks...................................................................... 17

1.2.2 Damage recognition and chromatin remodeling................................ 18

1.2.3 Repair pathway choice ....................................................................... 20

1.2.4 Homologous recombination repair........................................................ 22

1.2.5 Non-homologous end joining............................................................. 24

1.3 Computational approaches to network analysis, interaction prediction and protein design

1.4 Focus......................................................................................................

2. In silico engineering of synthetic binding proteins from random amino acid sequences.

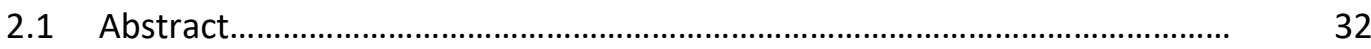

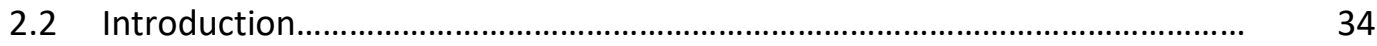

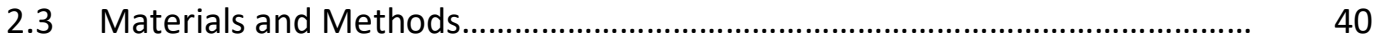

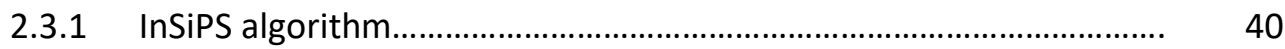

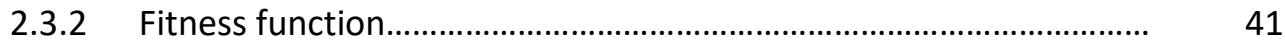

2.3.3 Target protein selection.................................................................. 42

2.3.4 Strains, vectors and media................................................................ 
2.3.5 Chemical sensitivity analysis............................................................ 44

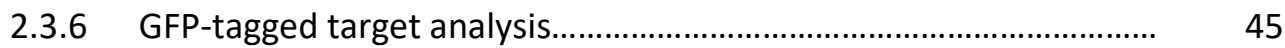

2.3.7 Yeast-2-hybrid construct preparation................................................... 45

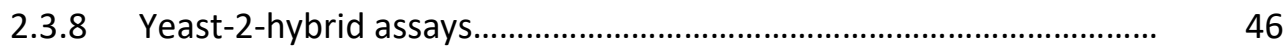

2.3.9 Protein purification and spot array analysis......................................... 47

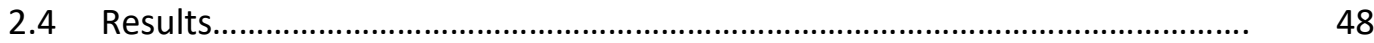

2.4.1 Synthetic protein design..................................................................... 48

2.4.2 In vivo analysis of functional activity................................................. 52

2.4.3 Yeast-two-hybrid binding analysis..................................................... 56

2.4.4 Peptide SPOT array analyses show that binding on targets occurs at predicted loci.......

2.4.5 Investigation of Psk1 binding motif.................................................... 61

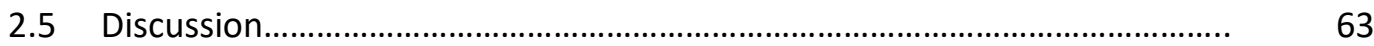

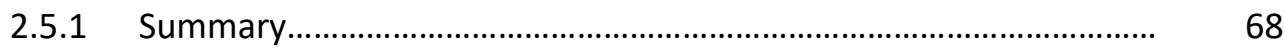

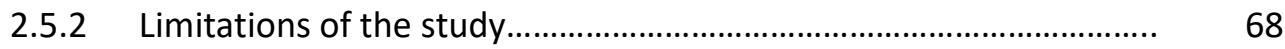

3. PAS kinase Psk1 influences non homologous end-joining fidelity................... $\quad 70$

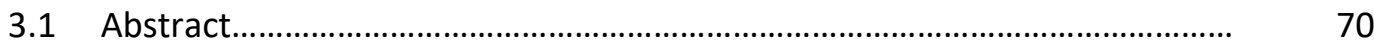


3.2 Introduction.

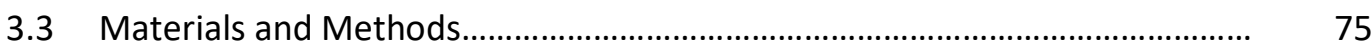

3.3.1 Yeast strains and plasmids................................................................

3.3.2 Spot test analysis of sensitivity to DNA damaging agents.................... 75

3.3.3 Plasmid repair assay............................................................................ 76

3.3.4 Chromosomal break assay.............................................................. 76

3.3.5 Genetic interaction analysis.................................................................. 77

3.3.6 Repair fidelity assay ....................................................................... 77

3.3.7 Growth curve analysis.................................................................

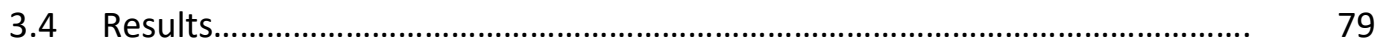

3.4.1 Identification of Psk1 as a novel repair factor..................................... 79

3.4.2 Analyzing the conditional genetic interaction profile of PSk1 under

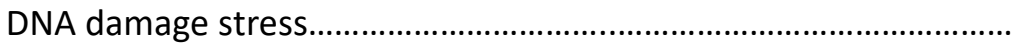

3.4.4 Repair fidelity............................................................................ 86

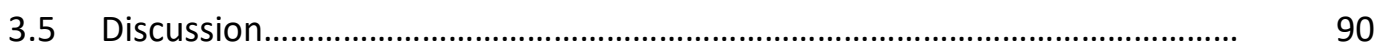

3.5.1 Summary................................................................................... 93 
4. Actin-related protein 6 (ARP6) influences double-strand break repair induced chromatin remodeling in yeast.....................................................................

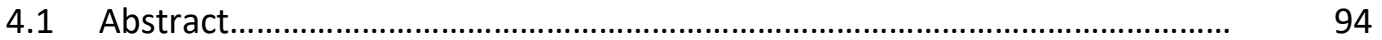

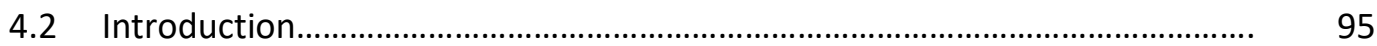

4.3 Materials and Methods................................................................................. 98

4.3.1 Yeast strains and plasmids................................................................ 98

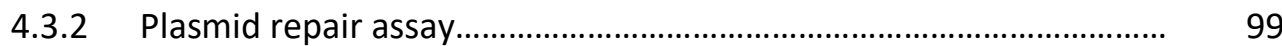

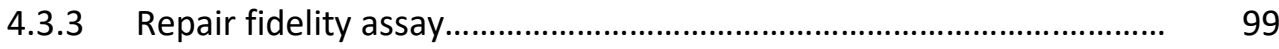

4.3.4 DNA damaging drug sensitivity analysis............................................... 100

4.3.5 Genetic interaction analysis............................................................. 100

4.3.6 Chromosomal repair assay ................................................................ 101

4.3.7 Homologous recombination assay...................................................... 101

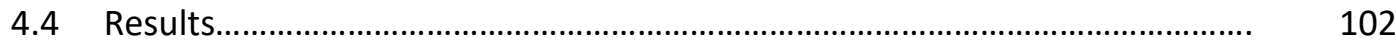

4.4.1 Assessment of ARP6 function in DNA repair...................................... 102

4.4.2 ARP6 effects repair fidelity.............................................................. 105

4.4.3 Interaction analysis suggests a role with DNA repair and chromatin remodeling 
4.4.4 Overexpression of ARP6 rescued four deletion mutants that were sensitive to DNA damaging drugs

4.4.5 ARP6 function appears related to the RSC-C chromatin remodeling complex.

4.4.6 Loss of ARP6 severely reduces the efficiency of homologous recombination

4.5 Discussion

4.5.1 Summary and future directions.

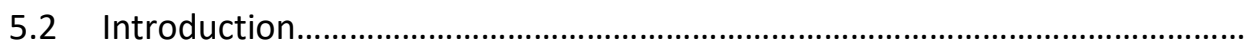

5.3.1 Homologous recombination assay. 
5.3.1 Homologous recombination assay..................................................... 126

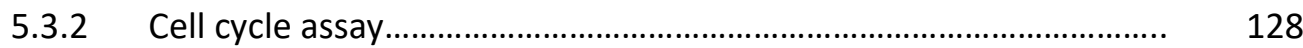

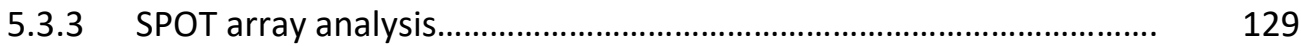

5.3.4 Genetic compensation.................................................................... 131

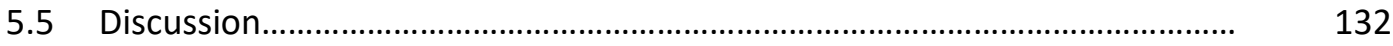

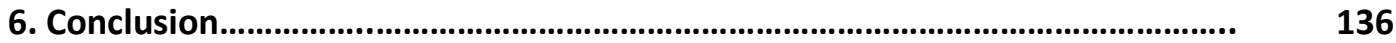

6.1 Concluding remarks........................................................................... 136

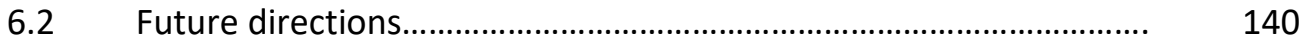

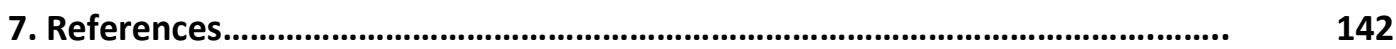

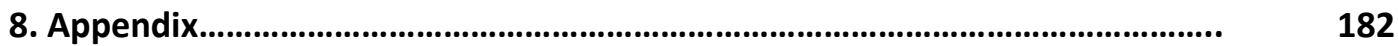

8.1 Additional data for chapter 2.......................................................... 182

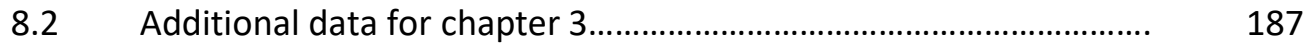

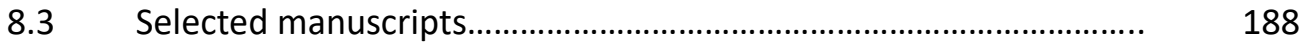




\section{List of Tables}

Table 2.1 Results of InSiPS against yeast target proteins............................ 50

Table $3.1 \quad$ Functional summary of PSK1 genetic interactors........................ 84

Table $3.2 \quad$ Reverse complementarity analysis.......................................... 87

Table 5.1 Predicting Def1 binding motifs.................................................... 127 


\section{List of Figures}

Figure 1.1 Traditional approaches to systems biology ................................. 4

Figure $1.2 \quad$ Genetic interactions............................................................... 8

Figure $1.3 \quad$ Model of NHEJ....................................................................... 27

Figure 2.1 Summary of methodology underlying chapter $2 \ldots \ldots \ldots \ldots \ldots \ldots \ldots \ldots \ldots . . . . . . . . . . . .33$

Figure 2.2 Overview of the InSiPS algorithm............................................... 36

Figure $2.3 \quad$ Tuning of InSiPS fitness function............................................... 49

Figure 2.4 Phenotypic and GFP assays testing anti-target protein function. 54

Figure 2.5 Yeast-2-hybrid analysis of target/anti-target binding.................. 57

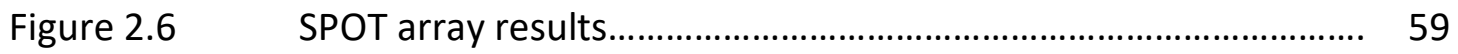

Figure 2.7 Characterization of Psk1 interaction motif and binding affinity. 62

Figure $3.1 \quad$ Analyzing the influence of PSK1 on DNA repair........................... 80

Figure 3.2 Genetic interaction network from SGA analysis......................... 82

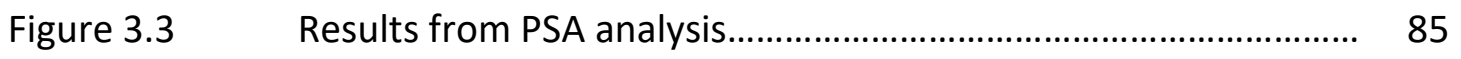

Figure 3.4 Examination of the functional relationship between RAD27 and

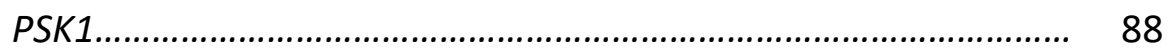

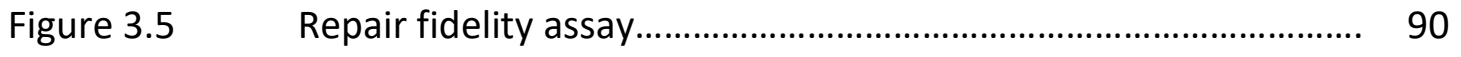

Figure $3.6 \quad$ Proposed model for Psk1 in NHEJ........................................... 92

Figure 4.1 Multiple assays indicate a role for ARP6 in NHEJ......................... 104

Figure 4.2 Loss of ARP6 increases repair fidelity........................................ 106 
Figure 4.3 Genetic interaction profile for ARP6......................................... 108

Figure 4.4 Analysis of ARP6 interactions with chromatin remodeling complexes involved in DNA repair......................................... 112

Figure 4.5 Arp6 protein-protein interaction network............................. 119

Figure $5.1 \quad$ Homologous recombination assay....................................... 125

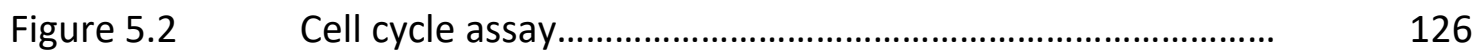

Figure 5.3 SPOT array indicates binding between Yku80 and Def1...... 127

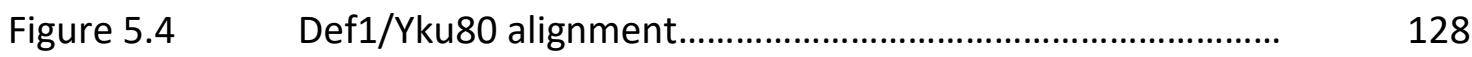

Figure $5.5 \quad$ Genetic compensation analysis........................................... 130 


\section{Abbreviations}

\begin{tabular}{|c|c|}
\hline$A m p^{r}$ & Ampicillin resistance \\
\hline AP & Apurinic \\
\hline BER & Base excision repair \\
\hline CPD & Computational protein design \\
\hline DDA & DNA damage array \\
\hline DDR & DNA damage response \\
\hline D-loops & Displacement loops \\
\hline DNA & Deoxyribonucleic acid \\
\hline dNTP & Deoxyribonucleotide triphosphate \\
\hline DSB & Double strand break \\
\hline GAL & Galactose \\
\hline GDA & Gene deletion array \\
\hline GI & Genetic interaction \\
\hline INO80-C & INO80 complex \\
\hline InSiPS & In Silico Protein Synthesizer \\
\hline IR & Infrared \\
\hline $\operatorname{Kan}^{r}$ & Kanamycin resistance \\
\hline Leu & Leucine \\
\hline MMEJ & Microhomology-mediated end-joining \\
\hline MMR & Mismatch repair \\
\hline MMS & Methyl methane-sulfonate \\
\hline MRX & Mre11-Rad50-Xrs2 - Homolog of mammalian MRN \\
\hline Nat $^{r}$ & Nourseothricin resistance \\
\hline NHEJ & Non-homologous end-joining \\
\hline
\end{tabular}




\begin{tabular}{|c|c|}
\hline ORF & Open reading frame \\
\hline PAS domain & Per-Arnt-Sim domain \\
\hline PCNA & Proliferating cell nuclear antigen \\
\hline PHLM & Phleomycin \\
\hline PIPE & Protein-protein interaction prediction engine \\
\hline PPI & Protein-protein interaction \\
\hline PSA & Phenotypic suppression analysis \\
\hline ROS & Reactive oxygen species \\
\hline RPA & Replication protein A \\
\hline SCID & Severe combined immunodeficiency \\
\hline CRISPR & Clustered regularly interspersed short palindromic repeats \\
\hline $\mathrm{RSC}-\mathrm{C}$ & Remodels the structure of chromatin complex \\
\hline SBP & Synthetic binding protein \\
\hline SCINEX-P & Screening for interactions between extracellular proteins \\
\hline SDL & Synthetic dosage lethality \\
\hline SGA & Synthetic genetic array \\
\hline SRYTH & Ste11p/Ste $50 p$ related yeast two-hybrid \\
\hline SSB & Single-strand break \\
\hline ssDNA & Single-stranded DNA \\
\hline SWR1-C & SWR complex \\
\hline TAP-MS & Tandem affinity purification + mass spectrometry \\
\hline Trp & Tryptophan \\
\hline Ura & Uracil \\
\hline UV & Ultraviolet \\
\hline WT & Wildtype \\
\hline $\mathrm{Y} 2 \mathrm{H}$ & Yeast-2-hybrid \\
\hline
\end{tabular}




\section{Introduction}

\subsection{Functional Analysis in Systems Biology}

When cellular components are isolated and subjected to targeted molecular biology studies, interesting mechanisms can be identified and novel insights into cell functionality can be produced. However, other important functions that these components perform only when operating within a cellular system may be overlooked. A systems biology approach, one that examines the function of cellular components within the context of a dynamic living system, can produce new insights into the activities of individual cellular components and the identification of potentially unexpected interaction networks. Systems biology, the study of the complex biological functions in relation to the individual molecular constituents involved ${ }^{1}$, utilizes the integration of data from multiple approaches to develop models and yield novel insights into gene function while striving toward an ultimate goal of developing mathematical models of living cells ${ }^{2}$.

A recent dramatic increase in large-scale biological experiments has resulted in an excess of genomic, proteomic, transcriptomic, and metabolomic data which is driving a growing need for i. organized and accessible databases, ii. novel analysis techniques and, importantly, iii. mechanistic studies to test and validate hypothesis generated from the data ${ }^{3-9}$.Together, the growing discrepancy between the exponentially increasing rate of data production and the ability of molecular biology to sufficiently interpret and translate the data into useful biological findings has driven an expansion of the field of systems biology ${ }^{10,11}$. 
One example of this excess data production is how the diminished cost and increased accessibility of next-generation sequencing platforms has resulted in an abundance of genetic sequencing data which includes genes that cannot be functionally annotated, limiting the ability to identify genes involved in a particular process or pathway of interest ${ }^{12,13}$. When it comes to infectious species, we now possess the ability to quickly and economically sequence the genome of a microbe of interest but the ability to translate this sequence information into meaningful insights into the understanding of gene function and ultimately a prediction of pathogenicity remains significantly more elusive ${ }^{14}$. Systems biology addresses problems such as these and aims to model how individual components within a cellular system interact together to affect the system as a whole. This already lofty goal is further complicated by the fact that cellular systems are dynamic and the interactions occurring within can shift dramatically when environmental conditions change.

Systems biology allows one to compare how cellular components function under normal cellular conditions to the functionality of said components when exposed to a specific threat or environmental shift. For example, when eukaryotic cells are faced with significant DNA damage, a highly-coordinated DNA damage response (DDR) is incited which activates a number of downstream pathways resulting in substantial alterations to interaction networks that persist until the threat has been resolved ${ }^{15}$. In this example, systems biology can be used to understand how the functional components of a cell adjust when the integrity of genetic information is jeopardized. The ability to 
model such situations and effectively interpret the data can be used to identify novel functions of genes/proteins that only occur under certain conditions. However, computational restraints and erroneous modeling due to incomplete or incorrect input data, particularly when using data from large-scale studies, can lead to inaccurate outcomes.

\subsubsection{Functional genomics}

The ability to predict novel gene function has become increasingly more feasible due to the methodical development of high-throughput platforms to analyze interactions or phenotypes on a genome-wide scale ${ }^{16,17}$. This field, collectively referred to as functional genomics, aims to define the functions of genes and proteins by intelligently interpreting the data produced by genome-wide analyses ${ }^{18}$. These global functional genomic and functional proteomic approaches have resulted in comprehensive databases for a variety of model organisms containing information on gene or protein functions, expression levels, protein localization, phenotypic data, interaction profiles, and more. A traditional functional genomic study follows a general "top-down" followed by a "bottom-up" pipeline to elucidating novel functions.

This generic functional genomic pipeline begins by analyzing large-scale datasets using network analysis to predict novel gene functions that can be tested in the lab (topdown). If a prediction turns out to be accurate and a gene appears to be involved in a specific pathway or process of interest, a subsequent "bottom-up" approach can be 
used to further characterize the specific role the gene plays in the context of the larger cellular system. Focused molecular experiments provide new insights into potential functions, which can be interpreted in the context of the known interaction network of the gene of interest (potentially under specific conditions) ultimately resulting in rewiring of known interaction networks (Figure 1.1).

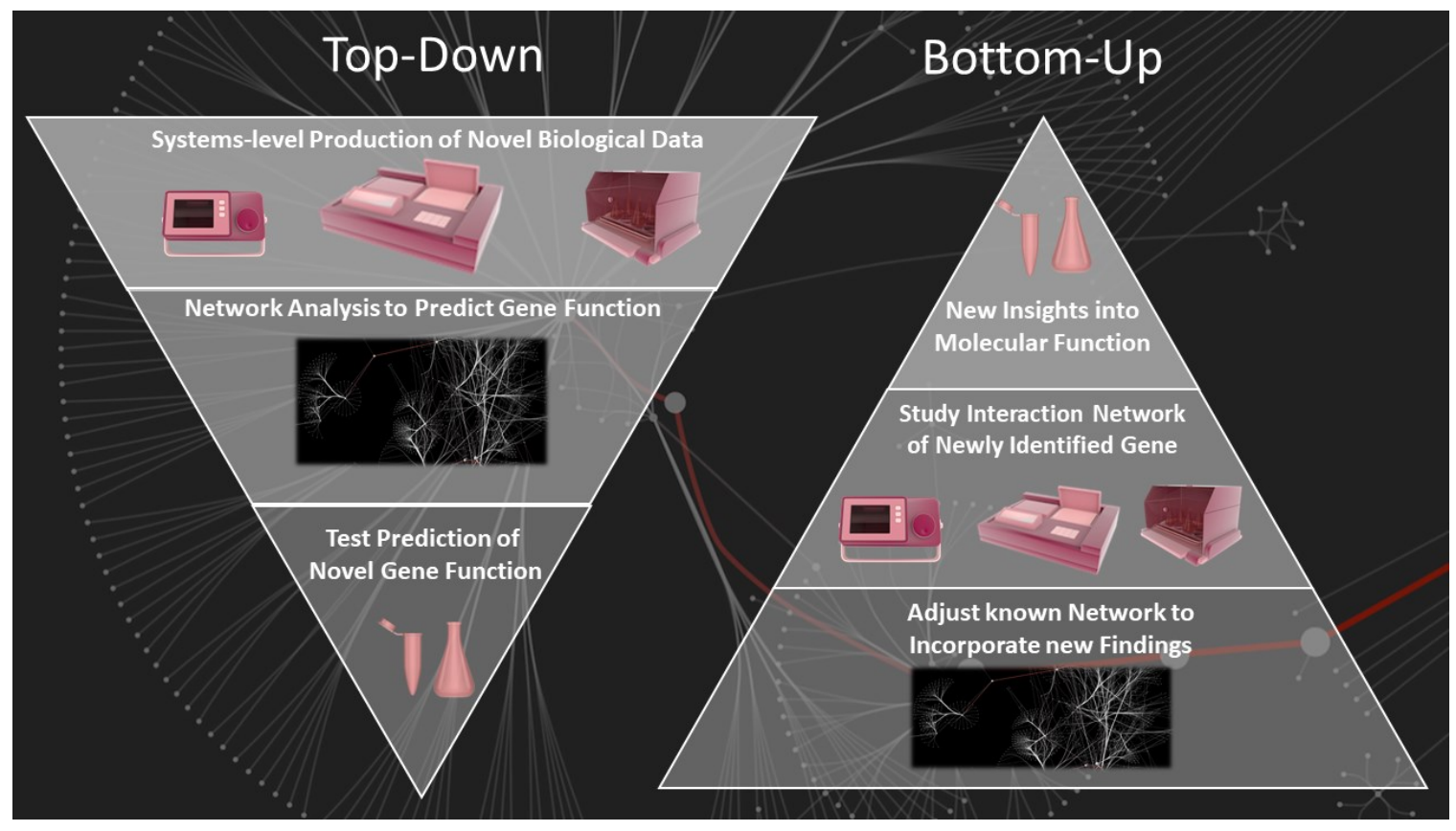

Figure 1.1: A model of two alternative approaches to systems biology. A "top-down" approach uses large data sets to infer functions that are tested in the lab. A "bottomup" approach interprets a novel finding in the context of known functional networks to incorporate new findings into our current knowledge of a system.

Several model systems are suitable for systematic genome-wide functional genomic studies but the budding yeast Saccharomyces cerevisiae in particular has excelled as a leading model for systems biology. Amenable to a wide variety of highthroughput techniques and readily cultured and modified in the lab, budding yeast is now arguably the best-characterized eukaryotic organism ${ }^{19}$. S. cerevisiae's place as a 
dominant model for eukaryotic systems is due in large part to several foundational projects that mapped the species including the Saccharomyces Genome Deletion Project, and multiple global protein-protein interaction (PPI), transcriptomic, and subcellular protein localization ventures.

\subsubsection{Large scale interaction analysis in yeast}

S. cerevisiae possesses many practical characteristics that make it amenable to large-scale analyses. It is cost-effective to maintain in either haploid or diploid form, undergoes fast and quantifiable growth in a variety of manipulatable environments either as individual colonies or batch cultures, and is relatively simple to modify genetically. Additionally, many fundamental biological pathways are well-conserved across eukaryotic taxa and yeast has proven to be an effective model for studying many of these by allowing for large scale analyses that are significantly more feasible when compared to using multicellular or more complex model systems ${ }^{19,20}$. In particular, the study of eukaryotic DNA repair has benefitted significantly from the advantages provided by the yeast model ${ }^{21}$. DNA repair pathways ${ }^{22}$ and related processes such as cell cycle progression ${ }^{23}$ and apoptosis ${ }^{24}$ are highly-conserved in eukaryotes.

Pioneering the field of systems-level analysis in yeast was the Saccharomyces Genome Project (1996) which comprehensively mapped the yeast genome and identified $\sim 6000$ open reading frames (ORFs) ${ }^{25}$. Following this genetic mapping, the Saccharomyces Genome Deletion Project successfully deleted $>96 \%$ of yeast ORFs and 
produced the first and only comprehensive eukaryotic gene deletion arrays (GDA) ${ }^{26,27}$. These collections, containing $\sim 6000$ heterozygous diploid and $\sim 5000$ viable haploid mutants are now available for a variety of genetic analyses and functional genomic studies. Commercially available genome-wide collections of; i. mutants ${ }^{27}$, ii. heterozygous mutant constructs, iii. temperature-sensitive essential gene mutants ${ }^{28}$ and iv. ORF over-expression vectors ${ }^{29}$ can be employed to study genetic interactions (GIs) under a variety of cellular conditions.

Over the past 15-20 years, yeast GDAs have been used for a range of genomewide phenotypic assays aimed towards increasing the overall understanding of eukaryotic biology and novel gene function. Additionally, the technology, has been used to study global cellular responses to various stressors and determine the mechanism(s) of action of biologically-active compounds ${ }^{30-32}$. Though these studies have varied widely in scope, there has been an over-representation of studies into DNA metabolism and repair ${ }^{26}$. Such biases are understandable when you consider the amount of data that can be produced from a single study. One endeavor to explore the changes that occur in genetic networks when faced with a panel of DNA-damaging agents resulted in $\sim 1.8$ million differential measurements for analysis ${ }^{33}$.

While yeast GDA and overexpression collections were being utilized to map genetic networks, several other "omics-level" platforms were concurrently being developed and employed to study protein expression ${ }^{34}$, determine subcellular localization ${ }^{35}$, investigate protein-interaction networks ${ }^{36}$, and develop a yeast 
metabolomics database ${ }^{37}$. The field of functional genomics benefitted significantly from the data produced from all of these studies, most of which is available in various publicly-accessible databases such as the Saccharomyces Genome Database (SGD) ${ }^{38}$, the Yeast Proteome Database (YPD) ${ }^{39}$, and the Yeast GFP-fusion localization database (YeastGFP) ${ }^{35}$. Data from large-scale genetic and protein interaction screens have proven to be beneficial in the search for novel gene functions both in the identification of new participants in a pathway and the characterization of gene function and as such will be focussed on in this introduction.

\subsubsection{Genetic interactions}

$\mathrm{Gl}$ analysis is a method to indicate functional redundancy across a genome by identifying genes involved in the same or similar biological process through an alternative pathway or mechanism. When the products of two independent genes or pathways have similar functions or produce the same outcome, the absence of one gene may be compensated by the presence of the other in a bidirectional manner. This relationship is referred to as epistasis, which, in this context, refers to the masking of a genetic phenotype by the phenotypic effects of other genetic alteration(s) to create phenotypes that do not resemble either of the single genetic manipulations ${ }^{40,41}$.

A GI is defined as an unexpected phenotype that occurs when a combination of genetic alterations produces more profound/unexpected changes compared to the phenotype of each individual alteration (Figure 1.2) ${ }^{42}$. In this way, Gls can indicate 
functional relationships between genes and often demonstrate a higher degree of complex interactions between different pathways ${ }^{43}$. Genetic interactions can be broadly classified as either positive (alleviating) or negative (aggravating).

a.

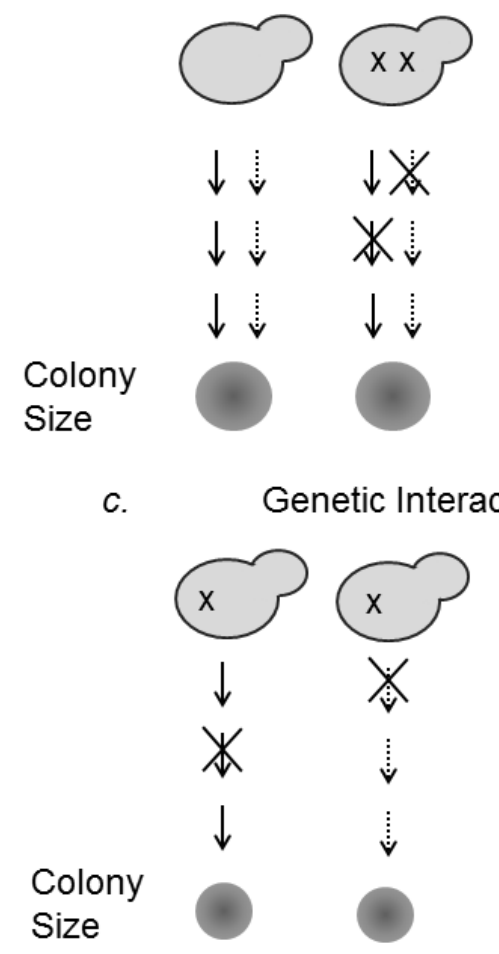

b. $\quad$ Non-interacting pathways
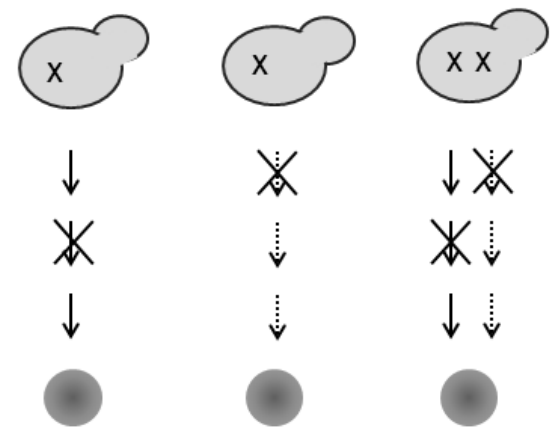

d. Chemical genetic interaction
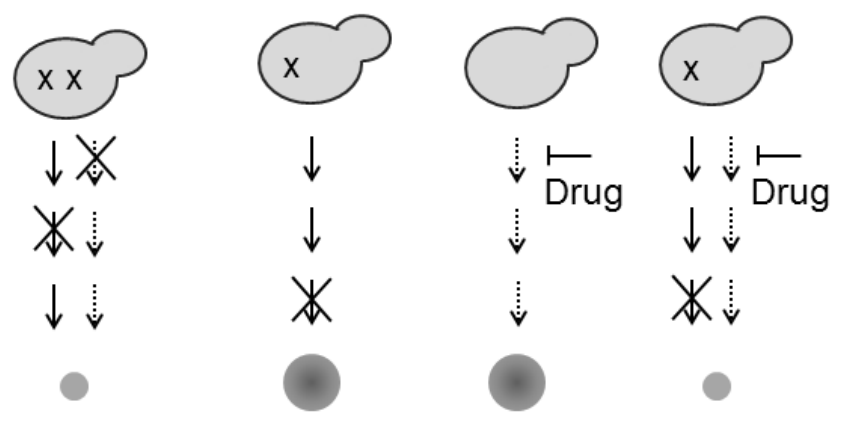

Figure 1.2: A schematic representing genetic interactions and chemical genetic interactions using colony size (fitness) as a measurable phenotype. Taken from Burnside et al, $2015^{44}$.

A negative genetic interaction (as seen in Figure 1.2) occurs when a combination of genetic manipulations decreases the fitness of a cell beyond the predicted multiplicative fitness of individual manipulations through a synergistic overlapping process. Alternatively, a positive Gl occurs when multiple genetic changes synergistically act to increase fitness beyond the predicted combined effects of each individual 
manipulation. One important instance of positive Gls is phenotypic suppression where the overexpression of one gene negates or "suppresses" the phenotype.

Screening for functional relationships on a genome-wide scale is a powerful tool for understanding genetic interconnectivity and providing clues as to how a cell functions as a system. The results of such studies demonstrate the extensive plasticity and redundancy apparent in a range of eukaryotes, and conditional-genetic studies accentuates biological complexity to reveal how compensatory genes act as buffers to perturbation. A project examining global genetic interactions in $S$. cerevisiae under antibiotic stress identified 170,000 functional relationships involving $75 \%$ of the genome ( 40 interaction partners/gene) ${ }^{45}$. The ability to study how genetic relationships shift under stress conditions is referred to as conditional-genetic analysis. The importance of a gene is relative to the current cellular environment such that certain non-essential genes may become conditionally essential when faced with a unique cellular stress.

Effective comparisons of genetic interaction networks between normal conditions and specific stress conditions can reveal significant alterations to networks that indicate conditionally important genes for a pathway of interest. Interaction hubs, genes with many interactions, undergo significant disruptions when perturbed by specific stressors such as the introduction of DNA damage stress. Such hubs will primarily contain known participants in the DNA damage response/repair pathways but can also used to identify novel participants in DNA repair. Isolating genetic interaction hubs that experience significant differential changes to interaction networks following DNA damage stress can 
identify novel DNA repair genes such as SLT2 ${ }^{15}$. SLT2 codes for a mitogen activated protein kinase (MAPK) known to contribute to the maintenance of cell wall integrity. Differential network analysis indicated that it undergoes significant changes to its interaction network following DNA damage stress via methyl methanesulfonate (MMS) exposure. This identified the $S L T 2$ gene product as a novel DNA repair factor that accumulates in the nucleus and regulates ribonucleotide reductase genes upon DNA damage stress. Generally, genes with similar interaction profiles often function within the same pathway or complex ${ }^{46}$. Information on how proteins interact physically can provide insights into novel gene functions in much the same way as genetic interactions do.

\subsubsection{Protein-protein interactions}

Proteins may function independently, but most often carry out their physiological functions through interactions with other proteins. A protein-protein interaction (PPI), the physical binding of two or more proteins due to electrostatic attraction and compatible biochemical properties encompasses a wide range of interaction types. Such interactions include everything from brief transient interactions such as receptor binding or signal transduction to highly stable or permanent interactions such as those seen in large macromolecular complexes ${ }^{47}$. Additionally, a PPI may involve complex 3-dimensional domain structures or only short motifs comprised of only a few amino acids ${ }^{48,49}$. Because PPIs vary significantly, attempts to map interaction networks often produce different results depending on the technique used or the cellular conditions ${ }^{50}$. Proteomic network analysis, the mapping of all known 
or predicted interactions within a cell, negates any classification of interaction type and instead and allows for the identification of functional associations on a global scale ${ }^{51}$.

The yeast model has arguably the best studied PPI network and has undergone multiple proteome-wide analyses both experimentally and computationally ${ }^{52}$. There are two primary experimental techniques that have been employed, yeast-two-hybrid $(\mathrm{Y} 2 \mathrm{H})$ analysis and tandem affinity purification followed by mass-spectrometry (TAP-MS), each with its own inherent advantages and disadvantages. Generally, TAP-MS co-purifies stable interaction partners of a protein of interest under native conditions which can help facilitate the identification of protein complexes while $\mathrm{Y} 2 \mathrm{H}$ is used to screen for binary PPIs in vivo under artificial conditions. The overlap between the results produced from each method is very low which is attributed to high rates of false positives and the fact that some methods may have difficultly detecting certain types of interactions ${ }^{53}$. Therefore, these two approaches are best thought of as complementary techniques rather than confirmatory.

The first attempts to explore large-scale PPI networks in yeast used a systematic $\mathrm{Y} 2 \mathrm{H}$ approach. The $\mathrm{Y} 2 \mathrm{H}$ technique is a genetic platform that can screen for binary interactions through the production of bait and prey fusion proteins such that if the proteins interact, they reconstitute a eukaryotic transcription factor and activate the expression of reporter genes ${ }^{54}$. In classical large-scale S. cerevisiae $\mathrm{Y} 2 \mathrm{H}$ screens, a "bait" protein is fused to the Gal4 DNA binding domain while the "prey" protein is fused to the Gal4 transcriptional activation domain. Both are cloned into expression plasmids and 
expressed in different yeast mating types ${ }^{55}$. Scaling up of the technology allows for a single bait to be screened against a pool of preys but this has not been sufficient to allow reliable all-to-all screens ${ }^{56}$. A systematic matrix approach crossed cells containing the bait and prey plasmids and selected for cells containing both plasmids. Three foundational large-scale yeast two hybrid screens reported between 2000 and 2001 successfully cloned over 6000 yeast ORFs and collectively reported over 5600 interactions ${ }^{55,57-59}$.

Many variations of the $\mathrm{Y} 2 \mathrm{H}$ approach have been developed such as the repressed transactivator system wherein an interaction results in the repression of Gal4 regulated genes ${ }^{60}$, the SCINEX-P (screening for interactions between extracellular proteins) system for screening extracellular proteins ${ }^{61}$, and the recently developed SRYTH method which relies on the Ste11/Ste50 interaction to activate the HOG pathway 62. Additionally, the $\mathrm{Y} 2 \mathrm{H}$ platform has been expanded to incorporate next-generation sequencing which has permitted the screening of large interaction networks. However, $\mathrm{Y} 2 \mathrm{H}$ is limited to detecting pairwise interactions and different tools are required for the detection of larger binding complex/networks.

TAP-MS is one such approach. It involves the tagging of individual protein "baits" at the genetic level with dual affinity tags (Staphylococcus protein A and calmodulin binding peptide) and a two-step purification process to isolate the tagged-protein along with its interaction partners ${ }^{63}$. The purified fusion protein and interaction partners are then separated (either using liquid chromatography or gel electrophoresis) into their 
individual components and identified through mass spectrometry. Despite limitations such as the tag interfering with protein function or complex formation and the purification process eluting weakly bound members, TAP-MS has proven to be a highly effective method for detecting yeast protein complexes under normal physiological conditions. Particularly, large scale affinity purification screens have been beneficial in providing new insights into DNA repair pathways ${ }^{64}$.

To translate findings from PPI screens to novel gene function predictions, the "guilt by association principle" is applied which asserts that a protein that generally interacts with other proteins involved in the same biological process or pathway ${ }^{65}$. PPI is a fundamental method of intracellular communication and therefore acts as an underlying tenet for studying system biology.

Identification of interacting protein partners with known functions, provides insights into the role of a protein within a pathway. In this study, we use data from a computational tool known as the protein-protein interaction prediction engine (PIPE) to infer protein function. PIPE is employed in multiple ways to predict PPIs in silico and scan for novel interactions not currently reported. In this way we have predicted new roles for genes by identifying novel PPIs involving known DNA repair proteins ${ }^{66}$. DNA repair is an essential process and identifying novel factors involved in important repair pathways is a popular area of focus for systems biologists. 


\subsection{DNA Damage and Repair}

The genetic code contains all the information needed to sustain life in a living cell. This heritable information must be protected to ensure coding genes can be transcribed, regulatory sequences remain functional and the structural integrity of DNA molecules is preserved so the entire genetic code can be passed on to future generations. However, cellular DNA is relentlessly assaulted and would not persist if it were not for a remarkable network of repair pathways that respond to DNA-damage.

There are two primary categories of DNA damage, spontaneous DNA damage that results from regular metabolic activities and environmentally induced damage that results from exposure to exogenous agents. The combined extent of daily DNA damage from these two sources is staggering ${ }^{67}$. For example, mammalian cells may experience up to $10^{5}$ spontaneous DNA lesions and $10^{5}$ environmentally-induced lesions per day ${ }^{68}$. The damage caused by either source can range from very minor damage that does not require immediate repair to severe forms of damage that threaten cell survival if not quickly resolved.

Spontaneous DNA damage may result from metabolic events such as the misincorporation of dNTPs, alkylation of DNA bases, deamination leading to baseconversions, loss of bases following depurination and collapsed replication forks ${ }^{68-70}$. Additionally, cellular production of reactive oxygen species (ROS) and oxidizing agents is 
a major source of spontaneous damage. Such species can produce up to 100 different types of modifications including both base lesions and damage to the DNA backbone ${ }^{71}$.

Some spontaneous lesions are less severe than others and the severity of a lesion can change dramatically if repair pathways are compromised or additional damage occurs during the repair process. For instance, when bases are incorrectly paired (mismatched), the mismatch repair (MMR) pathway degrades a section of the strand containing the error and allows the polymerase another attempt to accurately copy the template strand. However, dysfunctional MMR results in uncorrected bases which produce a "mutator" phenotype that can lead to mutations, microsatellite instability and eventually cancer in animals ${ }^{72}$. Depurination, the loss of a guanine or cytosine base to produce an apurinic (AP) site, is by far the most common spontaneous lesion at up to 10,000 lost purines per cell per day but it constitutes a relatively less severe form of damage ${ }^{73,74}$. AP sites can result from spontaneous processes such as alkylation and deamination or oxidation, as an intermediate step in the base excision repair (BER) pathway, or from damage by exogenous agents ${ }^{75}$. There are a variety of potential fates for AP sites. They can be accurately resolved via the BER pathway, they can lead to single-strand breaks (SSB) that compromise the phosphodiester backbone or, in severe cases, stall replication forks and lead to DSBs ${ }^{76,77}$.

In addition to the spontaneous damage previously discussed, several sources of environmentally induced damage also threaten the integrity of DNA. Environmental sources of DNA damage can be classified as either physical or chemical agents. Physical 
sources include ionizing radiation (IR) and ultraviolet (UV) radiation. IR causes a variety of lesions including direct damage to the backbone which can result is SSBs, DSBs or indirect damage to bases through the radiolysis of water to produce reactive species that can then damage bases ${ }^{78}$. UV radiation, primarily UV-B light with wavelengths from 280-315 nm, is a leading source of physical DNA damage whose influence has decreased in recent years following rebuilding of the ozone layer ${ }^{79}$. There are three primary lesions caused by UV radiation to two adjacent pyrimidine bases: i) cyclo-butane pyrimidine dimers which make up the majority of UV-induced lesions, ii) highlymutagenic 6-4 phytoproducts and iii) Dewar isomers ${ }^{80}$. These lesions can lead to mutations or cell death and are the primary reason for preventing unnecessary levels of exposure to UV waves from sunlight. In addition to physical damage from IR or UV radiation, many chemical compounds can produce a range of DNA lesions.

Exogenous chemical agents that damage DNA include crosslinking agents, alkylating agents, and intercalating agents among others. Cross-linking agents include a variety of chemotherapeutic compounds such as mitomycin which causes covalent links between bases on the same strands (intrastrand), across different stands (interstrand) or between proteins and DNA which can prevent progression of polymerases ${ }^{81}$. Alkylating agents such as MMS attach alkyl groups to various sites on DNA bases ${ }^{68}$. Intercalating agents insert themselves between bases which can challenge replication or produce additional secondary effects that result in damage. For example, phleomycin (PHLM) can intercalate DNA and also promote the production of free radicals that react 
to form a variety of DNA lesions, including DSBs ${ }^{82}$. There are other compounds that indirectly produce DNA damage. Hydroxyurea $(\mathrm{HU})$ depletes the dNTP pool and will eventually result in the collapse of replication forks and the induction of DSBs ${ }^{83}$. Events that lead to DSBs are very important to understand as these lesions are a major threat to DNA integrity and cell survival.

\subsubsection{Double-strand breaks}

A DSB, the cleavage of both strands of the phosphodiester backbone, results in a complete structural severing of the DNA molecule. DSBs can be extremely complex lesions as each broken strand can possess unique biochemical characteristics such as the presence of adducts and may require significant alteration before attempting to repair the break. Additionally, structural severing of a chromosome can result in translocations or the rearrangement of large segments of chromosomal DNA and even a single DSB can lead to cell death if misrepaired ${ }^{84}$. For these reasons and more, DSBs are considered the most serious form of DNA damage.

DSBs represent only a small fraction of DNA lesions. Mammalian cells experience approximately 10 DSBs per day compared to the roughly 100,000 adducts caused by sunlight alone ${ }^{68,85}$. However, the high mutagenic potential of DSBs ${ }^{84}$ is a significant cause for concern, particularly in human health. Defects in the pathways responsible for DSB repair can lead to cancer onset ${ }^{86}$, premature aging ${ }^{87}$, neurological disorders ${ }^{88}$, and immunodeficiencies ${ }^{89}$. Although DSB repair is best understood in humans, model 
organisms, specifically S. cerevisiae, have been invaluable in providing novel insights through these highly-conserved pathways ${ }^{90}$.

Two categories of repair pathways are able to resolve DSBs. Homologous recombination (HR) repair, also referred to as homology-dependent repair or recombination repair, involves the exchange of DNA sequences that are homologous to the break site ${ }^{91}$. Alternatively, the non-homologous end-joining pathway (NHEJ) directly ligates broken ends without utilizing a homologous template following various levels of processing ${ }^{92}$. DSB repair requires a highly coordinated response that involves efficient damage recognition, appropriate repair pathway choice, and effective communication between repair factors. These topics will be discussed in the following sections.

\subsubsection{Damage recognition and chromatin remodeling}

Sensors of DSBs must continually scan the genome performing the sensitive task of detecting broken DNA strands. They can both identify DSBs quickly and initiate a damage signal that can be amplified along a transduction cascade resulting in a global cellular response. Mre11 functions as the primary DNA damage sensor but the Remodels the Structure of Chromatin complex (RSC-C) also contributes to initial damage sensing ${ }^{93}$. DNA checkpoint proteins Mec1 and Tel1, functional homologs of human ATR and ATM, are activated following the detection of damage and initiate the DNA damage response (DDR). Mec1 is an essential protein and several damage sensors, including the PCNA-like complex (Rad17-Ddc1-Mec3), Dbp1, Lcd1, and RPA can contribute to Mec1 activity ${ }^{94,95}$. 
Ddc2, an ATRIP ortholog is capable of Mec1 activation independent of Ddc1 and Dbp196. The MRX complex (Mre11-Rad50-Xrs2) plays several roles in the early stages of DSB recognition. The FHA domain of the Xrs2 subunit is responsible for activating Tel1 and recruiting it to broken DNA strands ${ }^{97}$.

Following detection, Mec1 and Tel1, the key DNA damage response kinases, accumulate at break sites and begin phosphorylating multiple targets including the Rad53 and Chk1 checkpoint kinases and core histone protein $\mathrm{H}_{2 \mathrm{~A}}{ }^{94}$. Both Mec1 and Tel1 phosphorylate $\mathrm{H} 2 \mathrm{~A}$ on $\mathrm{S} 129$ to form the $\mathrm{\gamma}-\mathrm{H} 2 \mathrm{~A}$ DNA damage marker ${ }^{98}$. This phosphorylated $\mathrm{H} 2 \mathrm{~A}$ acts as a docking site for histone modifiers such as Arp4 and Esa1 and recruits various chromatin remodeling complexes involved in DSB repair. In preparation for repair, DNA attached to adjacent nucleosomes is unwound to allow repair proteins access. HR repair requires significantly more chromatin modification than NHEJ as longer tracts of DNA are involved in the repair process. Three complexes are primarily responsible for chromatin remodeling following a DSB event: INO80-C, SWR1-C and RSCC $98-100$.

Each of the three repair-related chromatin remodeling complexes promote unique repair dynamics. The ATP-dependent INO80-C plays a predominant role in the repair of DSBs at replication forks and promotes end-resection to induce HR-dependent repair ${ }^{101}$. SWR1-C facilitates recruitment and binding of the YKU heterodimer to the break site in preparation for NHEJ ${ }^{102}$. Both INO80-C and SWR1-C facilitate the damage-induced exchange of $\mathrm{H} 2 \mathrm{~A}$ with its variant $\mathrm{H} 2 \mathrm{~A} . \mathrm{Z}$ which relaxes the chromatin structure to provide 
access for repair proteins ${ }^{100,103}$. In addition to SWR1-C and INO-80, the RSC-C chromatin remodeling complex plays various roles in both $\mathrm{NHEJ}$ and $\mathrm{HR}$ repair including detecting breaks, signaling damage checkpoints, and facilitating nucleosome restructuring ${ }^{93,104}$. Chromatin remodeling activities following DSB detection have some influence on repair pathway choice. For example, Rad9 (homolog of human 53BP1) interacts with dimethyl histone $\mathrm{H} 3$ adjacent to DSB break sites in $\mathrm{G} 1$ to prevent end resection and push repair towards homology-independent repair ${ }^{105}$. However, several other more influential factors must be considered, primarily the current stage of the cell cycle and the availability of a homologous template sequence. Multiple regulatory mechanisms work to ensure that DSBs are repaired by the most appropriate repair pathway given the current cellular environment to prevent mutagenic events or complications that lead to cell death ${ }^{106}$.

\subsubsection{Repair pathway choice}

A more complete understanding of how cells determine which DSB repair pathway to employ following a DSB is now beginning to emerge. Generally, eukaryotes prefer the less error prone HR pathway, but a homologous template is not always available. In yeast, NHEJ is possible at all stages of the cell cycle but is generally restricted to $\mathrm{G} 1$ while $H R$, the dominant mechanism, is preferred from S-G2. HR is almost exclusively responsible for repair of collapsed replication forks, the leading endogenous cause of DSBs ${ }^{107}$. The mechanisms that underly this decision-making 
process remain unresolved. The accepted model is that the two pathways "compete" for the break site and that certain activities destine the break for a specific mechanism.

The level of end-processing appears to be the major determinant for dedicating a DSB to either HR or NHEJ repair. The YKU complex (Yku70/Yku80) has extremely high affinity for broken DNA ends and when bound, prevents resection and promotes direct ligation of the broken ends ${ }^{92}$. HR requires significant resection of the $5^{\prime}$ end resulting in a single-stranded $3^{\prime}$ tail that can initiate a search for homology. HR is the dominant repair mechanism in yeast and this resection activity is downregulated during $G 1$ and generally restricted to the $S$ and $G 2$ phases when a homologous template is available ${ }^{108}$.

The MRX complex (Mre11/Rad50/Xrs2) is primarily responsible for the initial 5' resection activity generating single-strand filaments ${ }^{107}$. Since the binding of YKU prevents this resection, it must be removed should the cell determine to repair via HR. This step is one of the most important in repair pathway choice and appears to be influenced by the activity of the Tel1 damage transducer kinase. After Tel1 is recruited to the site of the break, the Xrs2 subunit of the MRX complex activates Tel1 which in turn phosphorylates the Sae2 endonuclease ${ }^{109}$. Together, Sae2 and Mre11 promote an additional endonuclease, Ex01, which is required for the longer resection needed to complete HR. It is proposed that Exo1 directly completes with YKU and is responsible for its removal to allow HR to proceed ${ }^{97}$. Cdk1 and other cyclin-dependent kinases play a role in communicating between repair factors and cell cycle processes namely DNA replication and chromosome segregation ${ }^{110}$. Cdk1 accumulates at DSB break sites and 
phosphorylates multiple targets including Fun30, Sae2 and Dna2 playing an important role in promoting the DNA damage response and regulating end resection ${ }^{111}$. Cdk1 controls $5^{\prime}$ resection which is primarily performed by MRX and Sae2. Phosphorylation of Sae2 works to limit resection in G1 by removing MRX from DNA ends ${ }^{110,112}$.

To summarize, DSBs generally default to NHEJ due to the high affinity of YKU for broken ends which when bound protects the DNA from degradation. However, in yeast, HR generally outcompetes this initial designation and instead destines the break for resection and subsequent repair using a homologous template. In higher eukaryotes such as humans, NHEJ is the dominant mechanism due in part to the sheer size and structural complexity of the nuclear components which make the search for a homologous template increasingly more difficult. There are advantages and disadvantages to each pathway and ultimately, correct pathway choice is essential to minimize the rate of mutation and prevent aberrant cell death.

\subsubsection{Homologous recombination repair}

The use of a homologous template sequence during repair greatly decreases the rate of mutation at DSB sites. HR repair is often referred to as an error-free mechanism whereas NHEJ is referred to as error-prone. Recombination-based repair in S. cerevisiae is performed primarily by a set of proteins referred to as the "RAD52-epistasis group" which includes Rad50, Rad51, Rad52, Rad54, Mre11, Sgs1, Top3, Exo1 and others ${ }^{91}$. As mentioned previously, HR is initiated when 5' $-3^{\prime}$ resection of broken ends occurs. Initial 
resection is performed by the MRX complex (MRN in humans) in coordination with Sae2. However, two other functionally overlapping complexes also work to complete resection, namely the STR-Dna2 complex (Sgs1/Top2/Rmi-Dna2) and Exonuclease 1 (Exo1) ${ }^{113}$. In chemically complex breaks, MRX-Sae2 initially resects a few hundred nucleotides in each direction. The production of long tracts of single-stranded DNA (ssDNA) leaves genetic information vulnerable and thus, the long strands are coated to form active filaments.

The generated 3' single-strand overhangs are immediately coated by the singlestranded binding protein complex replication protein A (RPA) which protects the DNA from further degradation. RPA is then displaced and a Rad51 filament is formed in its place. The formation of the Rad51 filament is regulated primarily by key HR protein Rad52 and two Rad51 paralogs, Rad55 and Rad57 ${ }^{113,114}$. The Rad51 filament is exceptionally mobile and begins a search for homology within the yeast nucleus ${ }^{115}$. This homology search and subsequent strand-invasion by the Rad51 filament is performed in conjunction with Rad54 while Rad52 promotes the annealing of complexed ssDNA ${ }^{91}$. Following strand invasion there are four possible outcomes that can arise.

In certain cases, the invading strand can be synthesized beyond the break site and re-annealed to the other side of the break without a crossover product (synthesisdependent strand annealing) or the strand invasion event can induce the production of a new replication fork that completes synthesis to the end of a chromosome (break induced replication) ${ }^{113}$. However, the dominant mechanism of resolving duplexed DNA 
formed following strand invasion is the formation of a double Holliday junction which occurs when both broken ends are captured. These structures can be resolved by nucleases to produce crossover or non-crossover products.

Many of the molecular functions underlying HR were originally discovered in the yeast model and findings were later translated to other organisms. Similarly, we have learned a significant amount about the alternative pathway, NHEJ, using the yeast model. The next section will summarize the mechanism of NHEJ repair in yeast.

\subsubsection{Non-homologous end joining}

NHEJ is a robust pathway with extensive plasticity. Because the structure of DSBs can vary so widely and repair factors can arrive at different rates or in different patterns, NHEJ is best understood as an iterative process whereby multiple rounds of processing may occur before a break is finally resolved. For these reasons, the same break can be resolved in multiple different ways and potentially generate different resultant sequences each time it is repaired. Here we discuss the core NHEJ machinery that is essential for completing the repairing while acknowledging that many other additional factors, both known and unknown, are needed to achieve a functional repair mechanism.

NHEJ is focused around the YKU complex (Ku in humans) which functions as a central coordination center that translocates along broken strand acting as a docking station for the nucleases, polymerases, ligases and other factors that perform repair. 
The YKU complex, a heterodimer comprised of Yku70 and Yku80, is a ring-shaped structure found in high abundance within the nucleus. YKU has high affinity for doublestranded DNA ends and functions in telomere maintenance. There is also interplay between RPA and YKU wherein RPA binding can restrict YKU access to partially-resected ends but YKU can alternatively prevent further resection by Exo1 depending on the length and structure of the break overhang ${ }^{116}$. YKU and the MRX complex are amongst the first repair proteins to respond to a break and their antagonistic activities influence repair dynamics and pathway choice ${ }^{117}$. If YKU is not removed, other core NHEJ proteins will be recruited to the break site including DNA ligase IV/Lif1 (DnI4/Lif1). Binding of DnI4-Lif1 to YKU stabilizes the protein-DNA interaction and initiates the formation of the NHEJ complex that recruits additional end-processing factors ${ }^{118,119}$.

The NHEJ complex assembling around YKU functions to protect broken ends and recruit repair factors but does not keep ends in proximity to one another. The MRX complex largely performs this function by bridging the gap between and tethering ends through a long coiled coil structure in Rad50 ${ }^{120,121}$. MRX performs multiple roles in both major pathways of DSB repair including in the resection of ends for HR but in the context of NHEJ, it is believed to function through the Yku-Dnl4 complex. Mre11 is capable of both endo and exonuclease activity but this function is limited in NHEJ. All three components of MRX have DNA binding affinity however Xrs2, particularly the FHA domain of Xrs2, plays multiple roles in promoting repair activity. If resection is not 
required, Xrs2 promotes NHEJ through its interaction with Dnl4 and also functions to remove Ku at late stages to ensure repair continues to completion ${ }^{97}$.

Another core NHEJ repair factor is Nej1. In haploid cells, the loss of Nej1 is shown to decrease NHEJ efficiency 100 -fold and significantly impair the accumulation of Lif1 in the nucleus ${ }^{122}$. Binding of Nej1 to Ku, like Dnl4-Lif1, stabilizes the nucleoprotein structure ${ }^{123}$. Once ends are stabilized in the correct orientation, a variety of end processing pathways, many of which are redundant, work to generate compatible ends that can be ligated by Dnl4-Lif1.

The processing of broken DNA ends in preparation for NHEJ is accomplished by nucleases, polymerases and accessory factors. Several nucleases have been implicated in NHEJ including Exo1, Pol2, Mre11 and Rad27 ${ }^{97,124-126}$. Exo1 is believed to expose short sections of microhomology that can help to align strands prior to gap filling ${ }^{124}$. DNA polymerase II (Pol2) functions as 3' flap endonuclease that can remove aberrant 3' SSDNA strands that are produced during iterative processing ${ }^{126}$. The role that the Rad27 flap endonuclease plays in NHEJ remains unclear. Rad27 interacts physically with Dnl4, Pol4 and Nej1 and has been shown to be recruited to break sites through Nej1 activity but there may be functionally redundant nucleases that can perform this role ${ }^{118,125,127}$. During processing, gaps occur as a result of nuclease activity, the loss of nucleotides or from the annealing of short homologous regions. Highly adaptable polymerases are required to fill these unique gaps using unusual template structures. Members of the PolX family of polymerases generally fill this role. Mammalian cells have four members 
of this family (Pol $\lambda$, Pol $\mu$, Pol $\beta$, and TdT) while yeast has only one, the flexible Pol4 which possesses most of the properties of its mammalian counterparts Pol $\lambda$ and Pol $\mu$ that allow it to perform gap filling in NHEJ ${ }^{128}$. Pol4 is highly inaccurate but is able to function with limited homology beyond primer ends which may explain why it can promote chromosomal translocations when multiple breaks are present ${ }^{129}$. Once gaps are filled and strands sufficiently aligned, a final ligation step is required to complete the repair process.

The ATP driven DNA ligase complex interacts with all members of the core machinery. Dnl4 is inactive without its Lif1 component which functions as a mediator between the ligase and Xrs2/Nej1 ${ }^{121,130}$. In addition to these interactions, binding between the C-terminus of Yku80 and Dnl4 is important for efficient ligation activity. Nej1 also plays an important role in promoting ligation and when present allows for multiple ligations by a single Dnl4 molecule and reactivation following repair ${ }^{123}$. 


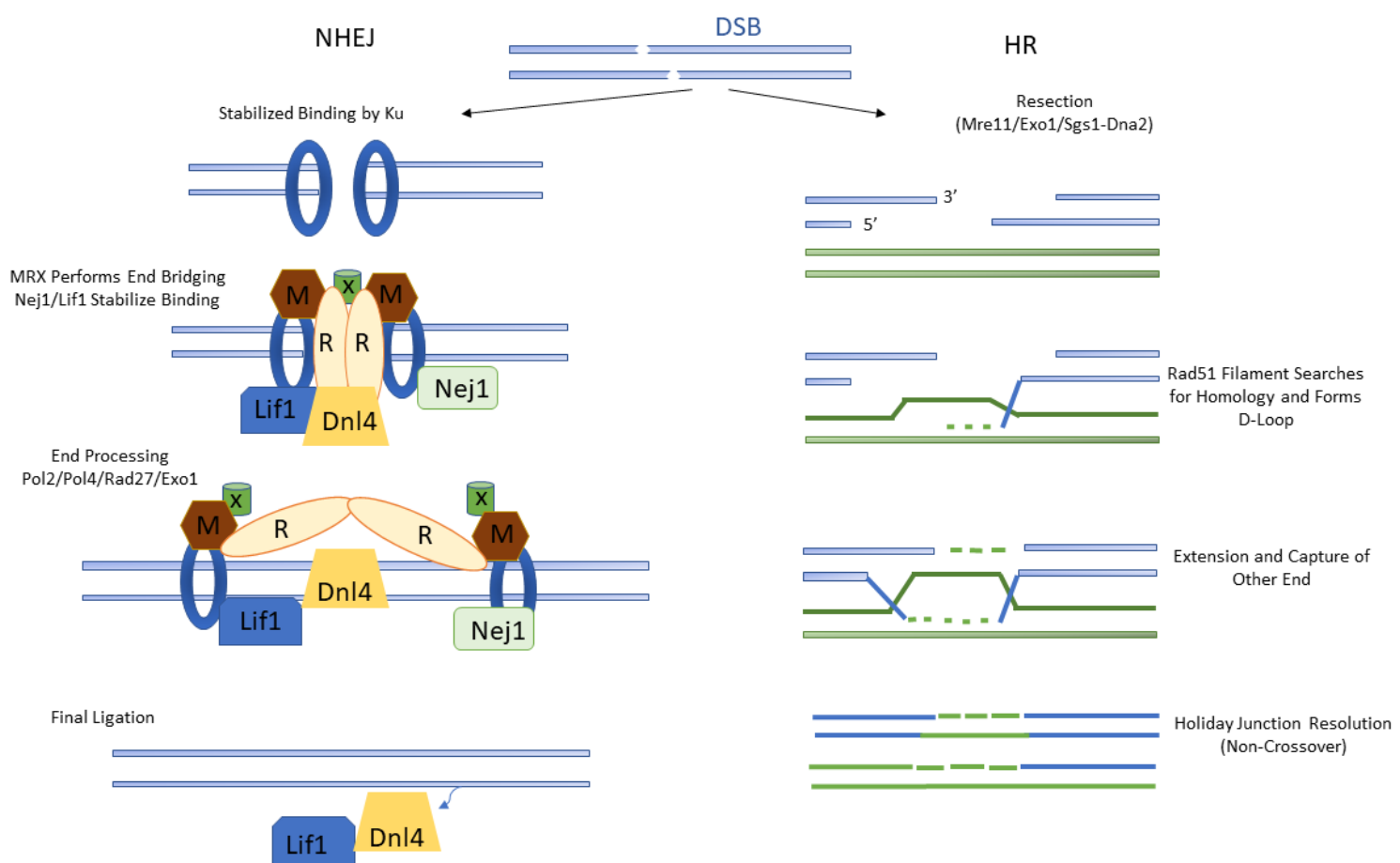

Figure 1.3: A simplified comparative model of NHEJ compared to HR. Ku binding (blue ring) recruits repair factors. MRX end-bridging keeps ends in close proximity while other repair factors process the ends prior to ligation by Dnl-Lif1. On the right, homologous recombination is performed beginning with end resection, strand invasion and D-loop formation followed by resolution.

The core NHEJ machinery (YKU, MRX, Nej1, Dnl4-Lif1) and various associated end processing factors are able to efficiently resolve a wide variety of breaks, but additional regulatory factors continue to be identified that contribute to the coordination and control of these processes. For example, spindle checkpoint kinases Bub1 and Bub2 were recently identified as novel repair factors that are recruited to break sites to phosphorylate effector kinase Tel and promote NHEJ and activation of the mitotic exit checkpoint ${ }^{131}$. Sub1 was also recently identified as an important factor that contributes to NHEJ efficiency in yeast and later observed to have a protective role in preventing damage caused by oxidative stress ${ }^{132,133}$. Importantly, here we see recent examples of 
how newly identified NHEJ repair factors can play significant roles in repair efficiency and fidelity despite not always functioning in coordination with the core machinery. Additionally, in both examples, the novel findings in a yeast model were translated into similar findings in mammalian systems with potential implications in the medical field.

This trend, coupled with the robustness of the NHEJ pathway and functional applications in medicine, immunology and gene editing through new technologies such as CRISPR-cas9 mean it is important to identify and study any additional factors that contribute to the function of NHEJ. The effective use of computational tools and available databases can allow for the identification of new NHEJ factors/mechanisms as we have done here.

\subsection{Computational approaches to network analysis, interaction prediction and protein design}

Several computational tools were used in this thesis project to predict novel gene functions, analyze findings, and design inhibitory proteins. Network analysis involves biological network integration, overlaying data from multiple databases to identify regions of high interconnectivity. GeneMANIA (www.genemania.org) is one such tool that allows for the imputation of gene lists. It searches co-expression data, physical and genetic interaction data, colocalization data, predicted protein-protein interaction data, and pathway and molecular interaction data to integrate the genes in your list with available data. GeneMANIA clusters your gene list and creates a network where genes on the list interact with each other as much as possible. Similarly, g:Profiler 
(https://biit.cs.ut.ee/gprofiler), another publicly available web server, characterizes gene lists and produces informative, visually appealing results. Benefits of g:Profiler include the ability to support ranked gene lists, recognition of multiple gene identifiers, and the ability to find orthologs and search for co-expressed genes from microarray databases. In addition to network integration tools, we also relied upon homology searches using BlastP, gene ontology analysis tools, the String ${ }^{\circledR}$ database and others to understand gene function. The PIPE protein interaction prediction tool, discussed elsewhere, was used to identify novel repair genes and interpret findings ${ }^{50}$. The second chapter of this thesis describes an endeavor to re-engineer PIPE to design novel binding proteins.

\subsection{Focus}

\section{Objective - 1: Contribute to the development of new tools to study functional genomics and systems biology}

This thesis relied on numerous bioinformatic tools developed by others as well as tools developed within our research group. It is important that new tools are continually developed to address deficits in a research field. One deficit identified over the course of this work was the lack of tools capable of designing novel proteins. As discussed in Chapter 2, most computational protein design (CPD) tools available modify known proteins and only a few can design new proteins de novo. We successfully developed a systems-based bioinformatics approach to engineer proteins that bind to a target of interest and avoid interacting with other proteins within the cellular 
environment. InSiPS was also able to engineer a protein that binds and inhibits novel NHEJ repair factor Psk1 identified herein.

\section{Objective - 2: Use systems biology approaches to study the process of DNA double strand break repair}

Understanding the process of DSB repair is important for fundamental biology, medicine, immunology, and genetic engineering. DSBs are extremely severe lesions that elicit a vast, interconnected global cellular response ${ }^{92}$. To study such a pathway, systems-based tools provide the best chance of understanding mechanisms that function within the repair networks. Using such tools, we were able to identify and study three DSB repair factors - PSK1, ARP6 and DEF1. These findings will help contribute to the continually evolving model of NHEJ and may prove beneficial to applications in the future. 


\section{Chapter 2: In silico engineering of synthetic binding proteins from random amino acid sequences}

\subsection{Abstract}

Synthetic proteins with high affinity and selectivity for a protein target can be used as research tools, biomarkers and pharmacological agents, but few methods exist to design such proteins de novo. To this end, the In-Silico Protein Synthesizer (InSiPS) was developed to design Synthetic Binding Proteins (SBPs) that bind pre-determined targets while minimizing off-target interactions. InSiPS is a genetic algorithm that refines a pool of random sequences over hundreds of generations of mutation and selection to produce SBPs with pre-specified binding characteristics. As a proof of concept, we design SBPs against three yeast proteins and demonstrate binding and functional inhibition of $2 / 3$ targets in vivo. Peptide spot arrays confirm binding sites and a permutation array demonstrates target specificity. Our foundational approach will support the field of de novo design of small binding polypeptide motifs and has robust applicability while offering potential advantages over the limited number of techniques currently available. 
Target proteins selected

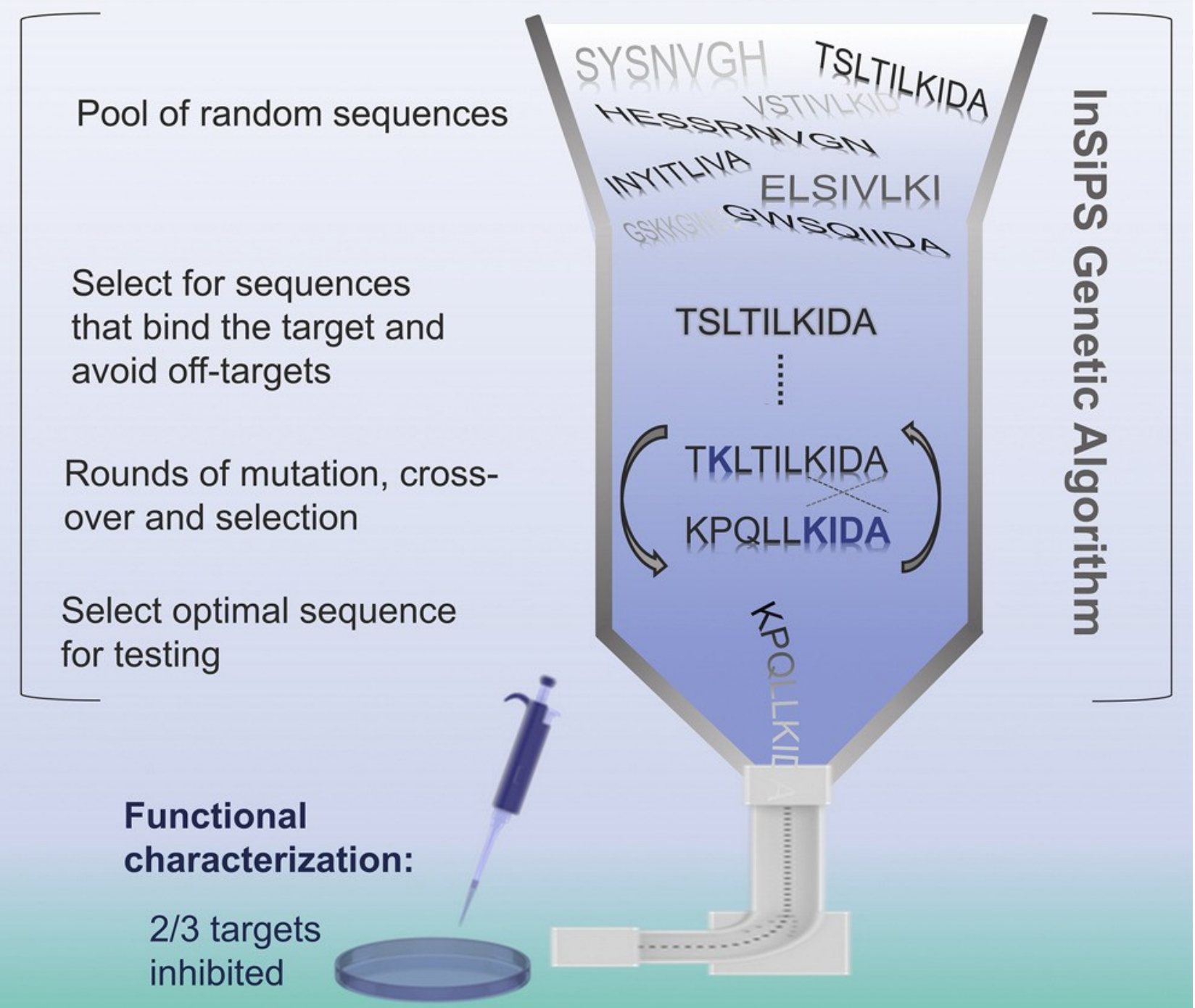

Figure 2.1: Graphical summary of the methodology underlying Chapter 2. 


\subsection{Introduction}

Proteins are diverse macromolecules that form intricate and complex proteinprotein interaction (PPI) networks through selective affinity binding. These properties have driven an expansion in the field of protein and peptide design over the past decade 134-137. Specifically, the ability to design synthetic proteins that can bind, label, and/or inhibit a specified target with high affinity are of primary importance and have the potential to replace antibodies and chemical compounds in a wide range of applications. Current methods to develop engineered binding proteins include peptide aptamer selection ${ }^{138}$, directed evolution of display systems ${ }^{139}$, and computational methods, the majority of which modify naturally occurring protein folds rather than designing novel structures ab initio ${ }^{140-143}$.

Computational protein design (CPD) can allow for the in silico evaluation of amino acid sequences on a scale that goes beyond the constraints of many laboratory approaches ${ }^{144}$. Natural proteins represent only an infinitesimal portion of potential functional sequences, limiting the scope of most current CPD techniques ${ }^{145}$. Many protein targets lie beyond the reach of natural protein folds or current approaches to developing binding peptides and searching randomized sequences space has been shown to successfully yield novel functional binding proteins ${ }^{146,147}$. It is thought that true large-scale de novo protein design can expand beyond the confines of biologically derived molecules into the vast space of "never-born proteins" 148,149 . This unexplored 
sequence potential coupled with the fact that many protein-based therapeutics have been shown to be effective and well-tolerated in clinical trials ${ }^{150,151}$ have made peptides a quickly expanding category of FDA approved drugs over the past twenty years ${ }^{152,153}$.

In addition to peptide-therapeutics, CPD has been used in recent years for developing ligand binding proteins ${ }^{154}$, nanobiotechnology ${ }^{155}$, enzyme design, and the development of antibody mimetics ${ }^{156}$. Much of the recent focus has been on developing proteins to replace targeted antibody therapies ${ }^{157,158}$. Rationally designed synthetic proteins with high affinity/specificity for a chosen target may become an important alternative to antibody-based biological drugs which experience numerous limitations including ineffective pharmacokinetics, a relatively large size, immunological complications, ethical questions and high production costs. Computational tools that can effectively design novel proteins specifically architected to interact with a wide range of targets are now beginning to emerge ${ }^{159,160}$.

We present a powerful massively-parallel computational tool that designs highaffinity binding proteins for a given target. This tool is the first of its kind as it employs a unique genetic algorithm, actively minimizes off-target interactions during the design process and does not employ docking models or require information on the 3D structure of the target. The InSiPS algorithm begins with a pool of random amino acid sequences and, over many generations of fitness-based selection followed by mutation and crossover events, converges on sequences that are predicted to interact with a specified target and minimize interactions with non-targets (other proteins in the 
environment) (Figure 2.2). InSiPS uses the co-occurrence of small interacting-motif pairs 50,66 to predict PPIs and intelligently design proteins with desired interaction profiles. In this way, previously "undruggable" proteins ${ }^{161}$, and those that lack a well-recognized binding pocket, may be targeted by this method.

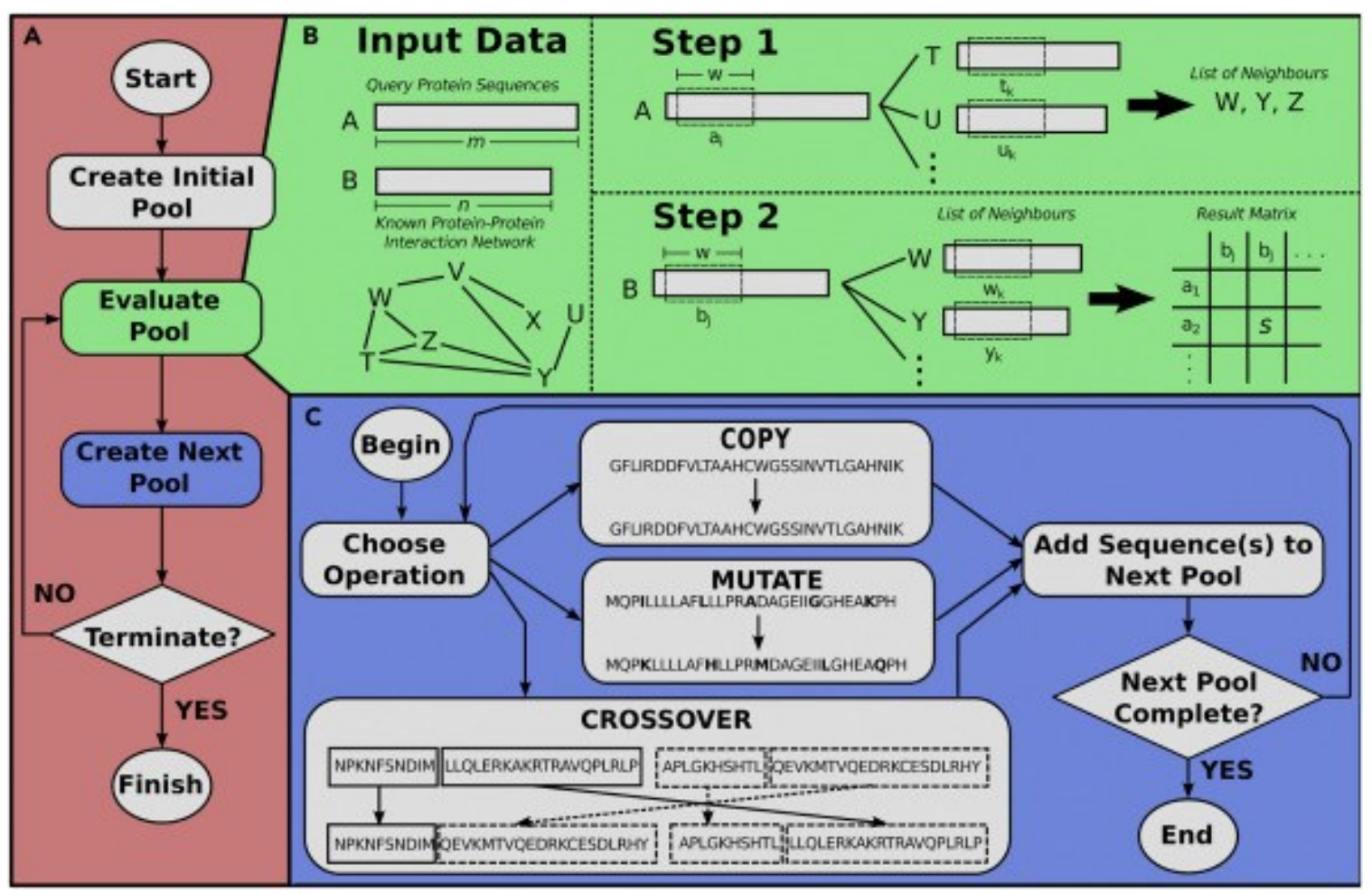

Figure 2.2: (A) An initial pool of random protein sequences 150 aa in length is created. Next, the primary loop is entered: sequences in the pool are evaluated, and subsequent generations are created. This process repeats for a minimum of 250 generations until a high-fitness peptide is produced. (B) PPI prediction. Sequences generated by InSiPS are evaluated using the Protein-Protein Interaction Prediction Engine (PIPE). PIPE requires a validated global PPI network as input. Step 1: protein $A$ is compared with all proteins in the known PPI network. A sliding window is used both on $A$ and the proteins in the PPI network until some segment of $A$, starting at position $i$, matches a segment of some protein $T$ in the network. All known interactors of $T$ (neighbors) are put into a list to be used in the next step. Step 2: protein $B$ is compared with the proteins in the neighbor's list in the same manner. When a segment of $B$, starting at position $j$, is found to match a segment of a protein from this list, the result matrix is incremented at position $(i, j)$. This matrix represents all the segments in proteins $A$ and $B$ that co-occur in experimentally 
validated PPIs and is used to predict if $A$ and $B$ interact. The interaction algorithm assigns a predicted interaction score between 0 and 1 . Any pair scoring over 0.51 is predicted to interact with a specificity of $99.5 \%$. The fitness of a protein sequence is calculated based on predicted interactions with targets-non-targets. (C) Generating the next generation of candidate sequences. First, a copy, mutate, or crossover operation is randomly chosen with a preset probability proportional to the fitness of a sequence as calculated in (B). This process is repeated until the next generation is complete. The algorithm is terminated after a minimum of 250 generations when the fitness score does not improve over 50 consecutive generations.

The InSiPS algorithm evaluates the predicted affinity and specificity (fitness) of hundreds-of-thousands of sequences over hundreds of generations, meaning upwards of a billion predictions are made in a single run. This scale is difficult to achieve when using competing methods that rely on detailed 3D protein configuration data ${ }^{162}$ and are thus limited by the computational restraints of working with docking models ${ }^{163}$. Other CPD methods have employed sequence-based approaches to design proteins. For example, Fisher et al, 2011 utilized binary patterning of alternating polar and non-polar residues to yield biologically functional proteins in Escherichia coli ${ }^{164}$. Keating and others have developed CLEVER and CLASSY, a method of cluster expansion which maps a complex function of atomic 3D coordinates from structure-based models of protein energetics to more simple linear functions of sequence. This change dramatically speeds up scoring and has been used to successfully design highly specific synthetic protein ligands against multiple basic region leucine zipper (bZIP) transcription factor families 165,166. However, InSiPS differs from these methods and other sequence-based approaches as it uses sequential optimization of binding via a genetic algorithm without 
any predetermined pattern or use of structural considerations, instead relying on conserved short, linear binding motifs.

Conserved short linear motifs are known to mediate PPIs in a manner that is unique from the more classically accepted interactions between large, rigid domain structures ${ }^{48,167}$ The more flexible linear motifs are ubiquitous across higher eukaryotes and are proposed to be capable of re-wiring PPI networks through the loss/gain of these functional modules ${ }^{168}$. Our algorithm uses primary protein sequences and experimentally validated interaction networks to screen for the co-occurrence of such motifs common to known protein pairs. This technique has been used to accurately predict global PPI networks in a variety of organisms including S. cerevisiae, Caenorhabditis elegans, Mus musculus and humans (with a precision of $82.1 \%$ ) ${ }^{50,66}$. As a proof of concept, we aimed to design SBPs that could functionally inhibit non-essential endogenous proteins in the yeast S. cerevisiae.

For ease of experimentation, 18 initial targets were chosen that fit our desired criteria of localizing to the cytoplasm, moderately size (200-1500aa) and stable abundance (500-5000 molecules per cell) in addition to possessing readily observable phenotypes when the encoding gene is deleted or the protein product is functionally inhibited. 3/18 targets with varying fitness scores were selected for wet-lab experimentation: (1) Psk1, a serine/threonine kinase which plays a role in regulating sugar metabolism, (2) Pin4, a protein involved in G2-M phase progression following DNA damage, and (3) Rmd1, a protein involved in meiotic nuclear division. The engineered 
SBPs were specifically designed to avoid interactions with $\sim 1700$ yeast cytoplasmic proteins (designated as non-targets). A length of 150aa was chosen for this project to ensure that the majority of predicted interaction motifs are included within the SBP sequence. Only a subsection of this 150aa polypeptide, which also contains a 6xHIS tag for affinity purification, is likely required for binding. We evaluated the ability of antiPsk1, anti-Pin4, and anti-Rmd1 to bind/inhibit their respective targets using a series of phenotypic assays and binding experiments. See Table S2-S3 for a complete list of InSiPS results for all target proteins considered in this study. Our results validate the ability of this approach to computationally engineer unique SBPs.

Because InSiPS does not begin with a template but rather a pool of random sequences, the algorithm has the potential to search sequence space beyond biological barriers and is not constrained to naturally occurring sequences. Moreover, because of efficient parallelization of the algorithm, hundreds of thousands of predictions can be made during each "generation" of the genetic algorithm, allowing strong selective pressure to be applied to maximize binding affinity and specificity. Ultimately, InSiPSengineered proteins may be useful for research, biotechnology, or as pharmacological agents. 


\subsection{Materials and methods}

\subsubsection{InSiPS algorithm}

The InSiPS algorithm aims to design protein sequences that are predicted to interact specifically with a given target. Formally, a problem is presented to InSiPS in the form of a target protein sequence and a set of non-target protein sequences (offtargets). For each protein design problem addressed in this manuscript, InSiPS was provided the sequences of the given target protein and the sequences of 1701 off-target yeast proteins known to localize to the cytoplasm. InSiPS then uses a genetic algorithm to design functional peptides. It starts with an initial population of 1000 randomly generated sequences.

Next, the fitness of each sequence is calculated based on the predicted interaction scores of the given sequence and the target/non-targets. Once all of the sequences in the current population have been evaluated, the next generation is created. Three operations are used to build the next generation: copy, mutate, crossover. The copy operation simply selects a sequence from the current generation and places it in the next generation. The mutate operation selects a sequence from the current generation and randomly mutates amino acids in said sequence with a fixed probability and transfers the resulting sequence in the next generation. The cross-over operation selects two sequences from the current population, chooses a random point at which to split the two sequences, exchanges the ends of the sequences and places 
the resulting new sequences in the next generation. Each operation needs to select at least one sequence from the current population, which is done randomly with each sequence's probability of being selected being proportional to its fitness. The probability of selecting the copy, mutation or cross-over operation as well as the rate of mutation can all be manually set. These parameters were set to $0.1,0.4,0.5$ and 0.05 , respectively, for all runs described here. This process of evaluation and creation was repeated for a minimum of 250 generations at which point the algorithm did not terminated until the fitness score of the best individual sequence observed did not improve for a further 50 generations. The three SBPs with the highest fitness score were chosen and re-run three times using random seeds and the highest scoring SBP was chosen for follow-up investigations.

\subsubsection{Fitness function}

InSiPS employs a well-established protein-protein interaction prediction tool known as PIPE (protein-protein interaction prediction engine) ${ }^{66,169}$ to predict the likelihood of sequences physically interacting with targets and non-targets. PIPE has successfully predicted proteome wide interaction maps in several species including $S$. cerevisiae, Schizosaccharomyces pombe, Caenorhabditis elegans, and humans at an extremely high specificity $(99.95 \%)^{66}$. The method is purely sequence based and has been independently shown to outperform competing PPI prediction methods in recall precision ${ }^{170}$ with a nearly negligible false positive rate of $0.05 \%$. 
To predict if a given pair of proteins interact, PIPE looks for motif pairs which co-occur in the query protein pairs and in the database of known interactions. If PIPE can build enough evidence of interaction via these co-occurrences, the protein pair will be predicted to interact. For full details on the PIPE algorithm, see ${ }^{50}$. To assign a fitness to a given protein sequence produced by InSiPS, PIPE is used to evaluate the predicted interaction profile of each sequence generated (likelihood of interacting with the target vs non-targets). The resulting prediction scores are combined into a single score using the InSiPS fitness function. This function rewards target binding and penalizes predicted off-target interactions. The fitness of a given sequence calculated as follows:

Fitness (seq) $=[1-\operatorname{MAX}(\operatorname{PIPE}($ seq, non-targets $)) \times$ PIPE (seq, target $)]$.

For each target, InSiPS generates an ordered list of sequences which maximize this fitness function. Importantly, this list generally includes several unique peptides that have limited homology to known proteins.

\subsubsection{Target protein selection}

18 endogenous yeast proteins with the following characteristics were chosen as experimental candidate targets;

i. localized to cytoplasm

ii. between $500-1500 a a$ in size

iii. moderate abundance $(3,000-10,000$ copies per cell)

iv. mutants demonstrate readily observable phenotypes (drug sensitivies/resistance) 
InSiPS generated an optimized anti-target protein for each of the 18 yeast cytoplasmic protein targets. The proteins with the top three highest anti-target scores (Rmd1, Pin4 and Psk1) were each re-run three more times using different random seeds. The best results from these runs were forwarded for experimental validation.

\subsubsection{Strains, vectors and media}

Output sequences from InSiPS for anti-Rmd1, anti-Pin4, and anti-Psk1 (Table 1) were synthetized by GeneArt ${ }^{\circledR}$, a division of Invitrogen/ThermoFisher and cloned into the pYES2 expression cassette. The open reading frame (ORF) of the synthetic proteins is under the control of a GAL1 promoter and a $6 \mathrm{X}$ His tag was added to the C-terminus. The cassette was also sub-cloned into pBI881 for use in yeast-two hybrid and affinity purification assays.

Drug sensitivity assays were performed using variations of S. cerevisiae S288C (MAT a orf $\Delta:$ :kanMX4 his3 $\Delta 1$ leu2 $\Delta 0$ met15 $\Delta 0$ ura3 $\Delta 0$ ), described in (Winzeler et al 1999). GFP experiments were performed in an EY0986 (MATa his3 $\Delta 1$ leu2 $\Delta 0$ met15 $\Delta 0$ ura3 $\Delta 0$ ) background (Huh 2013). Yeast-2-hybrid experiments were performed in MAV203 (MaV203 (MATanti leu2-3, 112, trp 1-901, his34200, ade2-101, gal44, gal804, SPAL10::URA3, GAL1::IacZ, HIS3UAS GAL1::HIS3@LYS2, can1R, cyh2R).

Strains were maintained in complete yeast medium YPD (yeast extract, peptone, $2 \%$ dextrose) or autotrophic medium (yeast nitrogen base, yeast extract, and an amino acid supplement lacking the appropriate amino acid (-leu lacking leucine, -ura lacking 
uracil, -trp lacking tryptophan, or -leu/-trp lacking leucine and tryptophan). Expression of synthetic proteins in yeast was achieved using medium supplemented with $2 \%$ galactose. Synthetic complete (SC) medium (yeast nitrogen base, yeast extract, and a complete amino acid supplement) was used as a complete medium when comparing to strains grown in autotrophic media. E. coli strains were grown in Lysogeny Broth medium with $50 \mu \mathrm{g} / \mathrm{mL}$ ampicillin for selection.

\subsubsection{Chemical sensitivity analysis}

Colony count analysis used S288C strains containing pYES2 plasmids expressing either anti-Psk1 or anti-Pin4 were grown to saturation in $5 \mathrm{~mL}$ of -ura liquid medium. A strain using an empty plasmid was used as a WT control. Cultures were then serially diluted in sterile distilled water from $10^{-1}$ to $10^{-4} .100 \mu \mathrm{L}$ of the $10^{-4}$ dilution was spread on YP-galactose agar plates for the control condition and plates were grown at $30^{\circ} \mathrm{C}$ for 48 hours. For the experimental condition, $100 \mu \mathrm{L}$ of the $10^{-4}$ dilution was spread on YPgalactose plates containing $65 \mathrm{ng} / \mathrm{mL}$ cycloheximide for the Pin4 experiment and for anti-Psk1, the plates were exposed to $30 \mathrm{sec}$ of UV radiation. Colony counts are represented as the ratio of colony forming units (CFUs) counted on the experimental condition plates over the number of CFUs on control plates normalized to the ratio of the WT + background plasmid strain in the same conditions.

For growth curve analyses, S288C strains containing pYES2 plasmids expressing either anti-Psk1 or anti-Pin4 were grown overnight to saturation in $5 \mathrm{~mL}$ of - 
ura+2\%dextrose. A strain using an empty plasmid was used as a WT and single deletion mutants as a positive control. Overnight cultures were diluted so that starting densities were consistent at $\mathrm{OD}_{600}=\sim 0.4$. Cultures were grown in appropriate medium over 8 hours with $\mathrm{OD}_{600}$ readings taken every 60 minutes. Drug conditions for anti-Psk1 were $0.02 \% \mathrm{v} / \mathrm{v}$ for methyl methanesulfonate (from Sigma Aldrich) and $1.0 \mathrm{mM} \mathrm{H}_{2} \mathrm{O}_{2}$. Drug conditions for anti-Pin4 were hygromycin $10 \mu \mathrm{g} / \mathrm{mL}$ and $0.1 \mathrm{mM}$ arsenite. $\mathrm{OD}_{600}$ values were normalized to control conditions.

\subsubsection{GFP-tagged target analysis}

S228C-derived strains with GFP-tagged targets were obtained from the yeastGFP collection analyzed to identify any alteration to the fluorescent signal when antitarget proteins were expressed ${ }^{35}$. Cultures were grown to saturation in $5 \mathrm{ml}$ of $\mathrm{YP}+2 \%$ glucose or -ura $+2 \%$ glucose. $10 \mu \mathrm{L}$ of each saturated culture was used to inoculate wells containing $190 \mu \mathrm{L}$ of either YPD or YP-galactose in microplates. Fluorescent determinations were made every 15 minutes using a BioTek FL600 microplate reader at $30^{\circ} \mathrm{C}$ shaking orbitally prior to readings and plotted over 6 hours.

\subsubsection{Yeast-2-hybrid construct preparation}

The ORFs of anti-Pin4 and anti-Psk1 were amplified from the synthesized pYES2 expression plasmids using primers with unique recognition sites to facilitate cloning into the prey (GAL4-TA) plasmid pBI-881 ${ }^{171}$. The ORF of Pin4 and Psk1 were amplified from commercially available BG1805 yeast overexpression plasmids obtained from GE- 
Dharmacon ${ }^{172}$ using unique restriction sequences and cloned into the bait (GAL4-DB) plasmid pB880. The anti-Pin4, anti-Psk1 inserts were cloned into pBI881 and Pin4 and Psk1 inserts were introduced into pBI880 using Sall-Notl recognition sequences. Primer sequences are found in the supplementary material (Supplementary Material 3).

Transformants containing pBI880 were selected on -leu plates and plated on -ura plates to ensure plasmids did not self-initiate. Double-transformants containing both the appropriate $\mathrm{pB} \mid 881$ and $\mathrm{B} \mid 880$ plasmids were selected on -leu/-tryp minimal medium plates.

\subsubsection{Yeast-2-hybrid assays}

Analysis of $\mathrm{Y} 2 \mathrm{H}$ interactions was performed using three reporter assays which measure expression of designated ORFs under the control of unique promoter sites influenced by the presence of a reconstituted Gal4 transcription factor. In MAV203, Gal4 activates transcription of URA3, HIS3 (competitively inhibited by 3-aminotriazole), and Beta-galactosidase. Firstly, to measure URA3 expression, a growth curve was constructed using liquid minimal medium lacking uracil and containing $2 \%$ galactose (ura/GAL). $5 \mathrm{~mL}$ cultures were grown to saturation in -leucine/-tryptophan and used to inoculate $40 \mathrm{~mL}$ of $-\mathrm{ura} / \mathrm{GAL}$ medium. Cultures were grown at $30^{\circ} \mathrm{C}$ shaking at $160 \mathrm{RPM}$ over 8 hours. Samples were taken every 60 minutes and OD 600 was read. Secondly, CFU counts on medium containing $25 \mathrm{mM} 3-\mathrm{AT}$ were performed. Again, $5 \mathrm{~mL}$ cultures were grown to saturation in -leu/-trp. $10^{-4}$ dilutions were plated on complete medium and 
10-3 dilutions were plated on SC+3AT. Results are expressed as the number of colonies on SC+3AT plates $(x 10)$ over the number of colonies on SC. Thirdly, $\beta$-galactosidase activity was measured using the liquid culture ONPG substrate assay with 30 minutes selected as an endpoint as previously described ${ }^{173}$.

\subsubsection{Protein purification and SPOT array analysis}

For purification, proteins were expressed in E. coli with a 6X-HIS tag were affinity purified using Ni-NTA agarose resin using a Bio-Rad gravity flow column according to manufacturer protocols. Individual peptides were synthesized at 0.1-mmol scale on a Multipep RSi peptide synthesizer (Intavis Inc.) using standard Fmoc ( $N$-(9-fluorenyl) methoxycarbonyl) chemistry. For fluorescein labeling, an appropriate amount of 5-(and6)-carboxyfluorescein succinimidyl ester was added to a peptide resin, and the coupling reaction was allowed to proceed for $1 \mathrm{~h}$ at room temperature. Upon cleavage of a peptide from the resin using trifluoroacetic acid, the fluorescein-labeled peptide was separated from the unlabeled peptide by HPLC on a $\mathrm{C}_{18}$ column. Identities of the peptides were confirmed by mass spectrometry.

Peptide SPOT arrays were synthesized as described previously ${ }^{174}$. All peptide SPOT arrays were blocked with 5\% BSA in TBST (0.1 M Tris- $\mathrm{HCl}, \mathrm{pH} 7.4,150 \mathrm{mM} \mathrm{NaCl}$, and $0.1 \%$ Tween 20 ) for $1 \mathrm{~h}$. Purified protein was added directly in the blocking buffer to a final concentration of $1 \mathrm{uM}$ and incubated with the SPOT array at room temperature for $1 \mathrm{~h}$. The array was then washed $3 \times 5$ min with TBST before a rabbit anti-His antibody 
(1:4000 dilution in TBST; Cat\# ab3553, Abcam) was added. The membrane was allowed to incubate at room temperature for $30 \mathrm{~min}$ prior to $3 \times 5$-min washes with TBST. After final $3 \times 5$-min washes in TBST, the SPOT arrays were visualized by enhanced HRP-based chemiluminescence.

Fluorescent polarization measurements were performed as described previously 174. A varied amount of a purified 6xHis-anti-Psk1 was titrated to a fluorescent peptide solution in $20 \mathrm{mM}$ PBS, pH 7.0, $100 \mathrm{mM} \mathrm{NaCl}$. The mixtures were allowed to incubate in a dark environment for 30 min prior to fluorescent anisotropy measurements at $20^{\circ} \mathrm{C}$. Binding curves were generated by fitting the isothermal binding data to a hyperbola nonlinear regression model using Prism 3.0 (GraphPad Software, Inc., San Diego, CA), which also produced the corresponding dissociation constants $(K d)$.

\subsection{Results}

\subsubsection{Synthetic protein design}

A preliminary run of InSiPS was used to evaluate initial SBP designs against 18 yeast targets. Our genetic algorithm assigns each candidate protein sequence a fitness (interaction) score between 0 and 1, balancing affinity for the target with specificity (Figure 2.1.A). For all cases, InSiPS was able to design SBPs with substantially stronger predicted affinity for the designated target than the highest likely "non-target" protein. Of the three target proteins chosen for wet lab experimentation, InSiPS successfully created proteins that had significantly higher affinity for the target than the highest non- 
target predicted score ${ }^{175}$. Additionally, all SBPs produced very low average non-target scores (Figure 2.1.B .and Table 2.1), highlighting the selectivity of SBPs, and showed limited sequence homology to known yeast proteins (Appendix 8.1). 
A

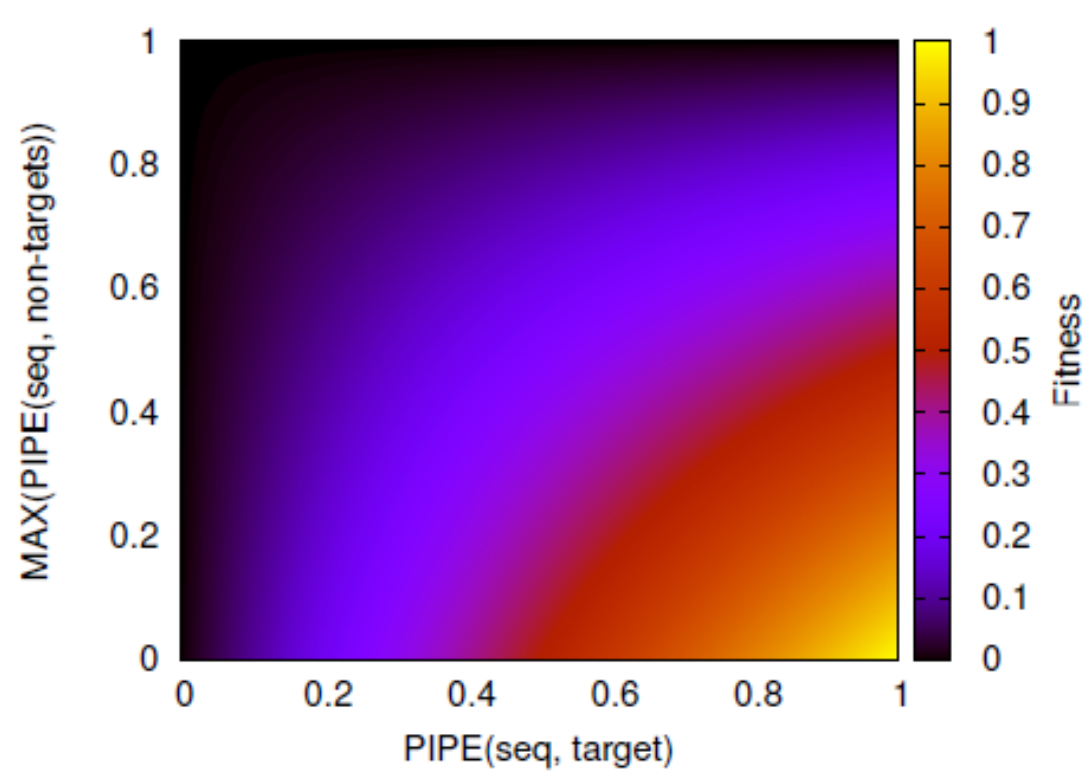

B

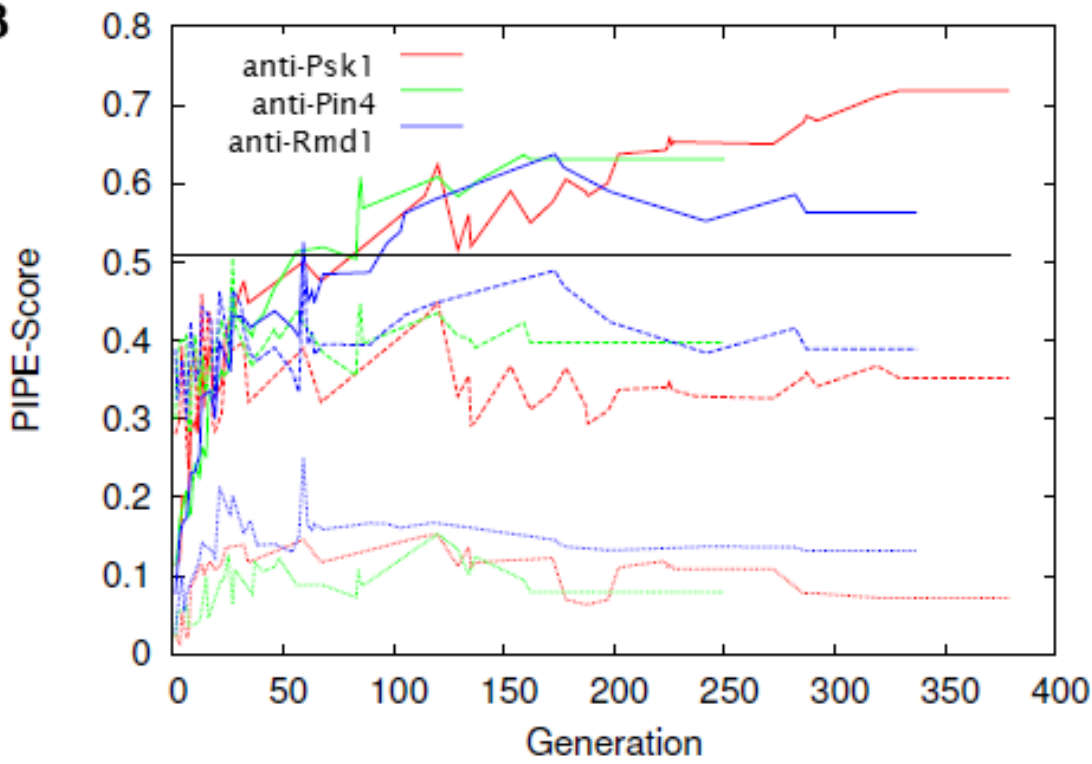

Figure 2.3: A) A heat map of the InSiPS fitness functions showing fitness as a function of affinity for the target PIPE(seq,target) and non-targets MAX(PIPE(seq,non-targets). B) The PIPE-predicted interaction score against respective targets (solid line), the highestscoring non-target (dashed-line) and the average non-target score (dotted line). The solid black line indicates the PIPE interaction threshold above which, an interaction is predicted to occur with a false positive rate of $<0.5 \%$. 
Of the three designed SBPs selected for wet-lab experimentation, anti-Psk1 demonstrated the highest fitness $(0.465)$ followed by anti-Pin4 (0.380) and anti-Rmd1 (0.344). All three anti-target proteins showed relatively strong interaction scores against their respective targets (Psk1 -0.718, Pin4 - 0.630, Rmd1-0.563). In all cases, the scores of all the non-target proteins are below the threshold of 0.51 at which the algorithm would predict an interaction, meaning no off-target interactions are predicted. InSiPS appears to work better for some targets than others.

Table 2.1: InSiPS Predictions Suggest High Affinity of SBPs for Target Proteins and No Predicted Off-target Interactions

\begin{tabular}{|l|l|l|l|l|l|}
\hline $\begin{array}{l}\text { Synthetic Binding } \\
\text { Protein }\end{array}$ & Fitness & $\begin{array}{l}\text { Target } \\
\text { Score }\end{array}$ & $\begin{array}{l}\text { Max Non-Target } \\
\text { Score }\end{array}$ & $\begin{array}{l}\text { Max } \\
\text { Non-Target }\end{array}$ & $\begin{array}{l}\text { Average } \\
\text { Non-Target Score }\end{array}$ \\
\hline Anti-Psk1 & 0.465 & 0.718 & 0.352 & Ubi4p & 0.072 \\
\hline Anti-Pin4 & 0.380 & 0.630 & 0.398 & Cdc39p & 0.0797 \\
\hline Anti-Rmd1 & 0.344 & 0.563 & 0.389 & Sec14p & 0.132 \\
\hline
\end{tabular}

Anti-Psk1 exceeds the other two anti-target proteins in terms of maximizing target score and minimizing of the max non-target score. InSiPS was able to effectively design binding proteins for all targets considered (Appendix 8.1) but only three targets were selected for wet-lab experimentation. To evaluate the novelty of the anti-target proteins, the sequences were compared against the yeast proteome using BlastP. The results show limited sequence similarity to yeast proteins (Appendix 8.1). Anti-Psk1 most closely resembles the known Psk1 interactor Mmp1 and aligns with 34\% coverage and a maximum 52\% identity over 38aa. Anti-Pin4 has sequence homology to a single yeast protein, Esl1, a known interactor of Pin4 with 29\% sequence identity over a region 
representing only $3 \%$ of the total protein sequence. Anti-Rmd1 was found to have significant sequence homology with two proteins, Sec14 with a maximum $52 \%$ sequence identity over 29aa and Pmt5 with a maximum 28\% sequence identity. However, neither of these proteins are known to interact with Rmd1.

\subsubsection{In vivo analysis of functional activity}

We hypothesized that the expression of our anti-target proteins may inhibit the function of the target proteins if biologically significant binding occurs in vivo. To this end, we expressed the SBPs in S. cerevisiae and performed three assays that examined conditional viability/growth rate, and effects on protein distribution (Figure 2.4). To test if the anti-Psk1 SBP can functionally inhibit Psk1, we induced oxidative stress and compared viability and growth rate to WT and $\triangle p s k 1$ strains as the loss of $P S K 1$ is known to increase sensitivity to UV light and oxidizing agents ${ }^{176}$. Anti-Psk1 expression phenocopies the $\Delta p s k 1$ mutant, consistent with inhibition of protein function (Figure 2.3). As seen in Figure 2.4.A,C, $\Delta p s k 1$ is sensitive to UV irradiation and exposure to $\mathrm{H}_{2} \mathrm{O}_{2}$, phenotypes that were also seen when anti-Psk1 is expressed. UV exposure decreased viability in $\Delta p s k 1$ by $85 \%$ and by $83 \%$ when anti-Psk1 is expressed. To determine if the sensitivity observed was the result of the anti-Psk1 protein and not other factors, we expressed anti-Psk1 in the $\Delta p s k 1$ strain and observed no significant alteration to viability. Growth curve analysis showed that exposure to $\mathrm{H}_{2} \mathrm{O}_{2}$ decreased the growth rate of cells expressing anti-Psk1 relative to WT cells which strongly resembles the $\Delta p s k 1$ 
phenotype (Figure 2.4.C). Additionally, expression of anti-Psk1 resulted in a significant change (net decrease) in the fluorescent signal of GFP-tagged-Psk1 protein suggesting possible aggregation or degradation of the target (Figure 2.4.E). 
A

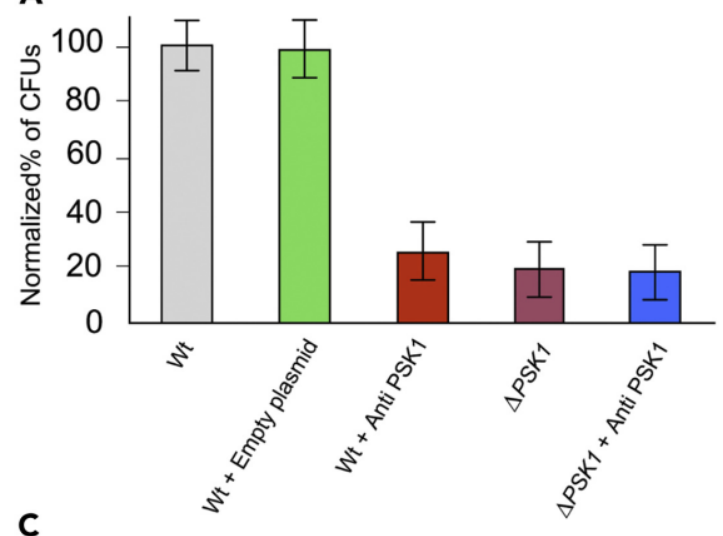

C

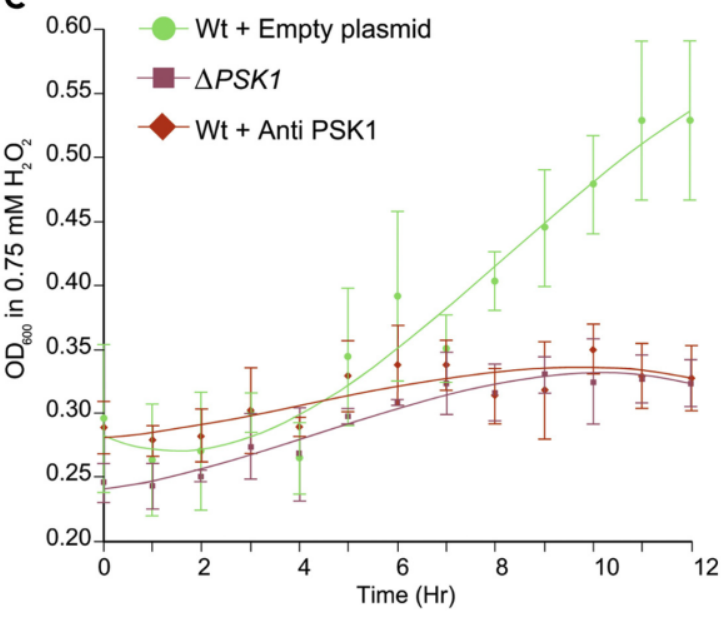

$\mathbf{E}$

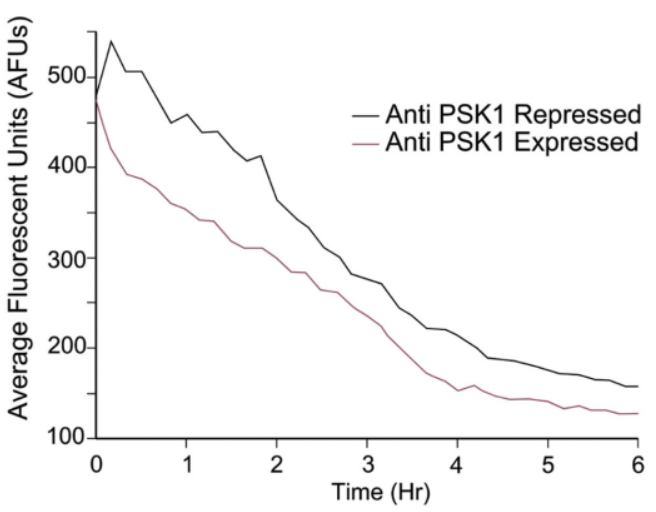

B
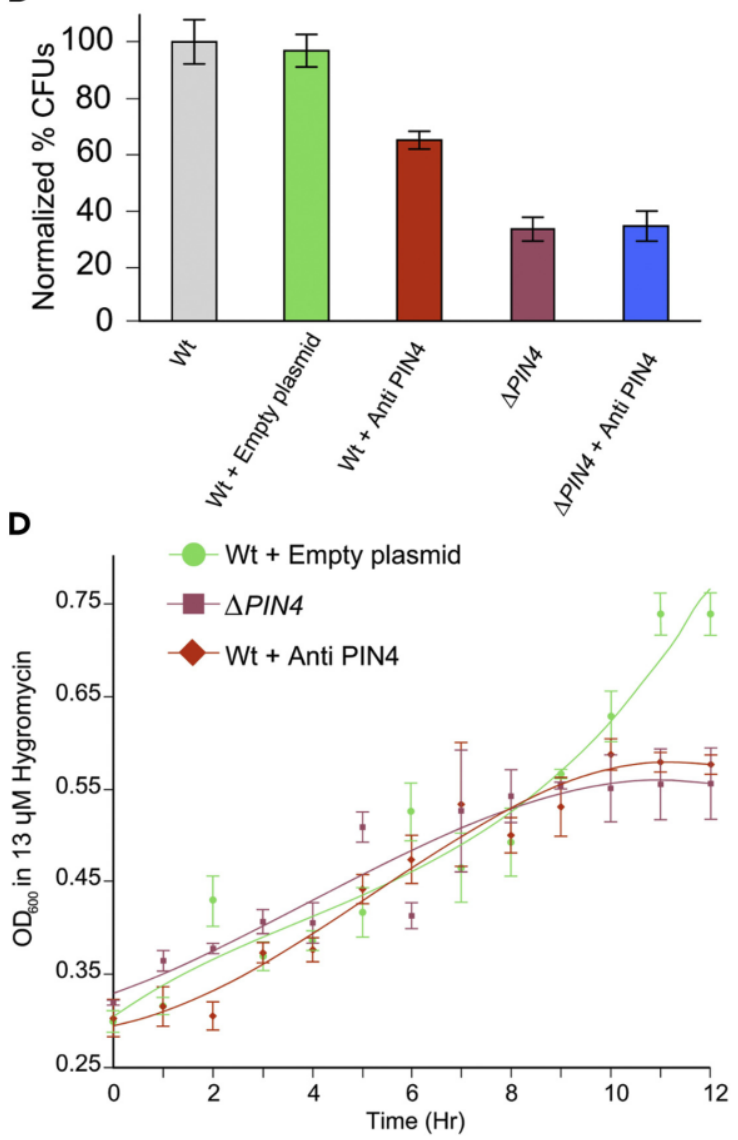

$\mathbf{F}$

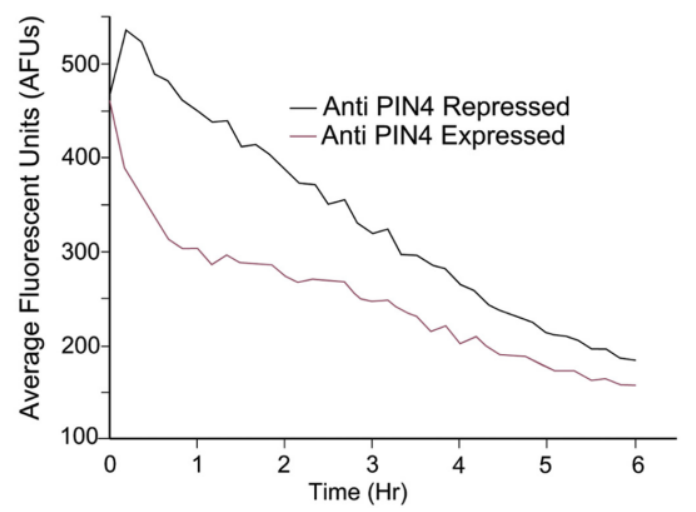

Figure 2.4: Strains expressing anti-Psk1 and anti-Pin4 can phenocopy deletion mutants of the target proteins and alter target protein expression/assembly. ( $A$ and B) Viability of cells under strain-specific stress condition shows that anti-Psk1 and anti-Pin4 expression can produce phenotypes that resemble loss of function mutants of the target proteins. ( $A$ and $B$ ) Average normalized colony-forming unit (CFU) counts from four trials are displayed as mean \pm SD. Stress conditions in trial were $(A)$ exposure to UV light for $30 \mathrm{~s}$ 
for the anti-Psk1 trial and (B) exposure to cycloheximide $(65 \mathrm{ng} / \mathrm{mL}$ ) for the Pin4 trial. (C and D) Expression of anti-target SBPs produces growth defects under strain-specific stress conditions that resemble deletion of the target. Three replicates of each culture or condition were grown for $12 \mathrm{~h}$ in liquid YPG (Yeast extract/Peptone/Galactose) + drug media and OD600 was measured hourly. This experiment was repeated three times. Error bars represent SD among replicates, and a polynomial line of best fit is presented. (C) $\Delta p s k 1$ sensitivity to $\mathrm{H}_{2} \mathrm{O}_{2}$ resembles the phenotype of strains expressing anti-Psk1. Cells were grown in medium containing $0.75 \mathrm{mM} \mathrm{H}_{2} \mathrm{O}_{2}$. (D) $\Delta$ pin4 sensitivity to $13 \mu \mathrm{M}$ hygromycin resembles the phenotype of strains expressing anti-Pin4. ( $E$ and $F$ ) Observed alteration of fluorescence profile of GFP-tagged targets when anti-target proteins are expressed. Aliquots of WT + anti-target protein cells from the same culture were used to inoculate complete medium with either $4 \%$ galactose (where anti-target SBP is expressed) or $4 \%$ glucose (where anti-target SBP is repressed), and overall fluorescent signal from three independent cultures for each condition were measured over time and normalized to the growth rate.

We performed the same assays as above to test for any functional inhibition of Pin4 by the anti-Pin4 protein. The deletion of PIN4 is known to cause yeast cells to become sensitive to inhibitors of protein synthesis such as cycloheximide and hygromycin $B{ }^{177}$. Culturing in the presence of $65 \mathrm{ng} / \mathrm{mL}$ cycloheximide decreased viability in $\Delta$ pin4 by $67 \%, \mathrm{WT}+$ anti-Pin4 by $34 \%$ and $\Delta p$ in $4+$ anti-Pin4 by $35 \%$ (Figure 2.3.B). Growth curves show similar sensitivity of WT+anti-Pin4 and $\Delta p i n 4$ to hygromycin B (Figure 2.3.D). These results show that anti-Pin4 sensitizes cells to translational inhibitors, suggesting functional inhibition of Pin4. Additionally, cells expressing antiPin4 become sensitive to arsenite in a manner similar to $\Delta p i n 4$ (Appendix 8.1). The expression of anti-Pin4 decreased the net fluorescent signal of GFP-tagged Pin4. Together these results suggest partial functional inhibition of target protein function. The third target selected for experimentation, Rmd1, was analyzed to detect if phenotypic changes can be produced through expression of the anti-Rmd1 protein. 
However, no significant alteration to conditional viability/sensitivity to $\beta$ -

mercaptoethanol or L-1,4-dithiothreitol exposure similar to $\Delta r m d 1$ was observed and no significant change to the fluorescent profile of GFP-tagged Rmd1 was detected when anti-Rmd1 was expressed. For these reasons, we chose not to experiment further using the anti-Rmd1 peptide as the aim of this project was to demonstrate functional inhibition through binding. This observation suggests that not all designed anti-target peptides are functionally effective.

\subsubsection{Yeast-2-hybrid binding analysis}

Together, the results displayed in Figure 2.4 suggest that anti-Psk1 and anti-Pin4 possibly bind to and alter the endogenous functionality of their respective targets. To confirm that binary PPIs between Psk1/anti-Psk1 and Pin4/anti-Pin4 occur in vivo, we performed a series of $\mathrm{Y} 2 \mathrm{H}$ assays. Three reporter genes were present in our $\mathrm{Y} 2 \mathrm{H}$ strain which are all induced by Gal4 reconstitution and three independent reporter assays were employed to test for interactions (see 2.3 Materials and Methods). In this way, the reconstitution of GAL4 by a physical interaction between the bait (target) and prey (antitarget) will induce growth on minimal medium lacking uracil, activate lacZ activity, and provide resistance to 3-AT. All three reporter assays showed binding signals indicating physical interactions between the two target/anti-target combinations in vivo (Figure 24). Figure 2.5.C indicates that binding affinity between anti-Pin4/Pin4 may be lower than 
the anti-Psk1/Psk1 affinity as this $\beta$-galactosidase assay is the most quantifiable of the three $\mathrm{Y} 2 \mathrm{H}$ assays employed.

A

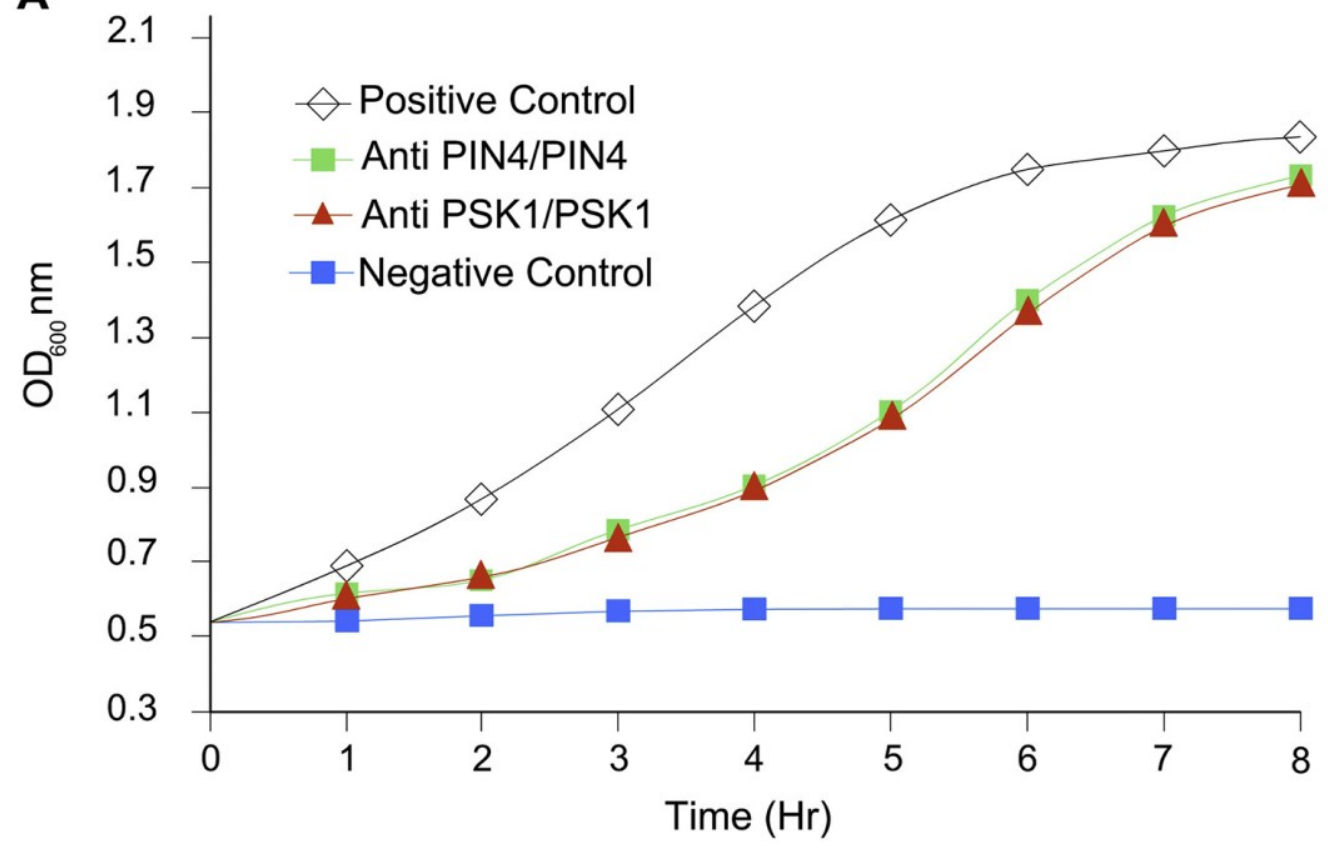

B

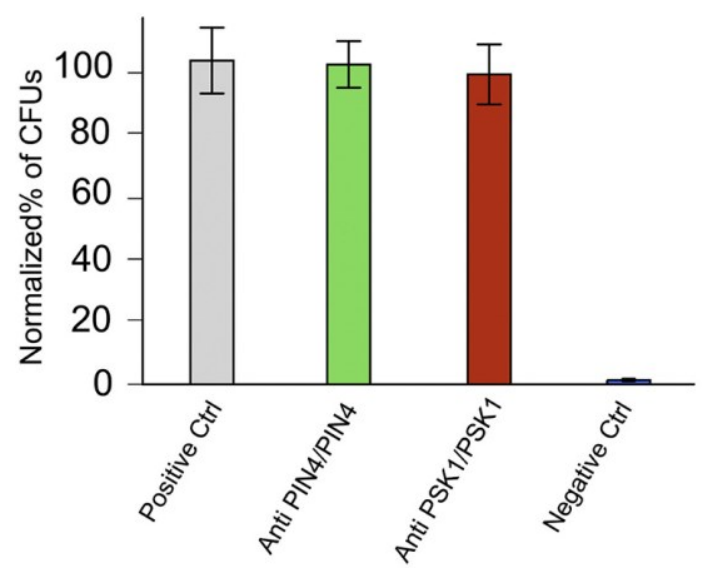

C

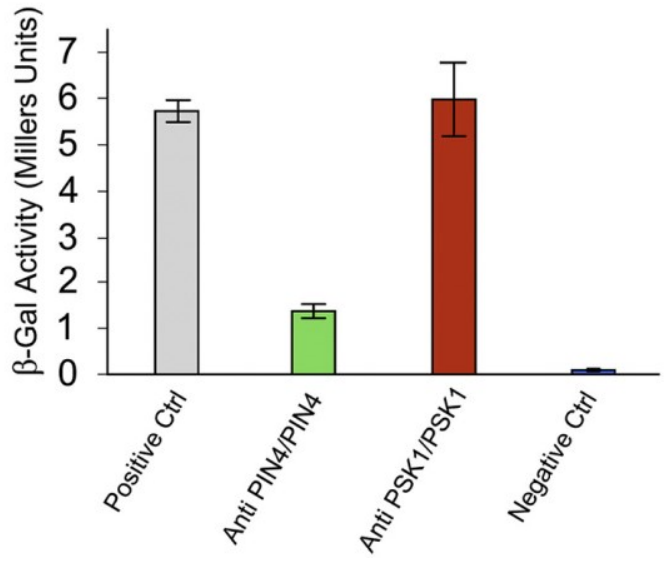

Figure 2.5: Yeast-2-hybrid analysis indicates direct physical interactions between target and anti-target proteins. ( $\mathrm{A}$ and $\mathrm{B}$ ) (A) Positive $\mathrm{Y} 2 \mathrm{H}$ results using uracil reporter assay. $\mathrm{A}$ growth curve in minimal medium lacking uracil shows that Pin4/anti-Pin4 and Psk1/antiPsk1 bait-prey combinations grow better than the negative control strain, indicating a PPI between bait and prey proteins through expression of the URA3 reporter. Triplicate trials produced similar positive results, but the results from a single trial are shown. (B) 
Positive $\mathrm{Y} 2 \mathrm{H}$ result for Pin4/anti-Pin4 and Psk1/anti-Psk1 bait-prey combinations based on resistance to 3-aminotriazole (3-AT). Normalized colony-forming unit (CFU) counts for triplicate trials on minimal medium lacking histidine $+25 \mathrm{mM}$ 3-AT resistance in test bait-prey combinations and in the positive control are presented as mean \pm SD. (C) Positive $\mathrm{Y} 2 \mathrm{H}$ result for Pin4/anti-Pin4 and Psk1/anti-Psk1 bait-prey combinations using a $\beta$-galactosidase reporter. Miller units are used to quantify $\beta$-galactosidase activity by measuring the hydrolysis of ortho-Nitrophenyl- $\beta$-galactoside (ONPG) spectrophotometrically. Relative $\beta$-gal activity (fold change) from triplicate trials is shown relative to negative control \pm SD. The Psk1/anti-Psk1 interaction produced a stronger signal than Pin4/anti-Pin4.

\subsubsection{Peptide spot-array analyses show that binding on targets occurs at}

\section{predicated loci}

Positive results in our $\mathrm{Y} 2 \mathrm{H}$ assays further support binding between target and anti-target proteins in a biological system. To probe these interactions in vitro and to further evaluate target interaction sites, a walking peptide spot array was used (Figure 2.6) ${ }^{174}$. Because InSiPS predicts regions on both the target and anti-target proteins responsible for binding, it provides a starting point to probe the binding regions. Using InSiPS-predicted interaction sites, we designed walking peptide arrays that probed the predicted interaction site of the target and flanking regions using 18aa motifs shifting at single amino acid intervals. As seen in Figure 2.6.A,B, very specific residues on both interacting partners are proposed to facilitate binding (dark green regions). Interactions between both anti-Psk1/Psk1 and anti-Pin4/Pin4 were shown to occur within or directly adjacent to PIPE-predicted interaction regions (Figure 2.6.C,D). 

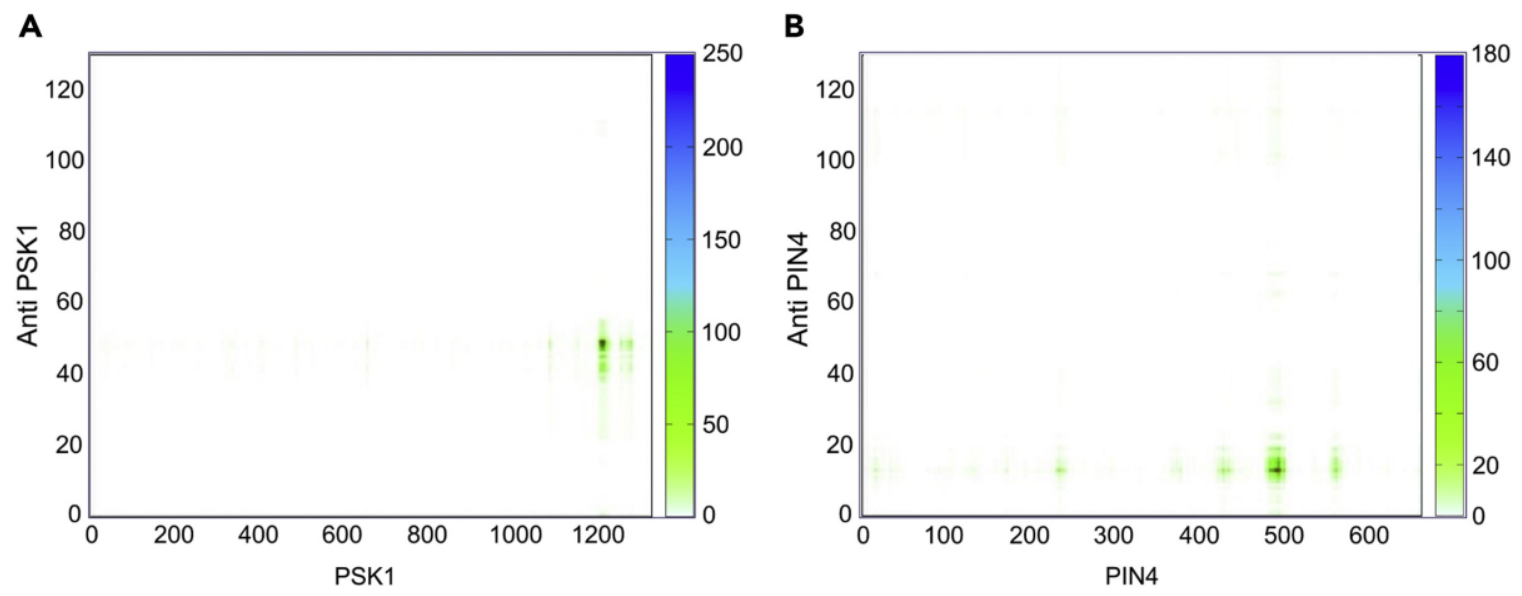

C

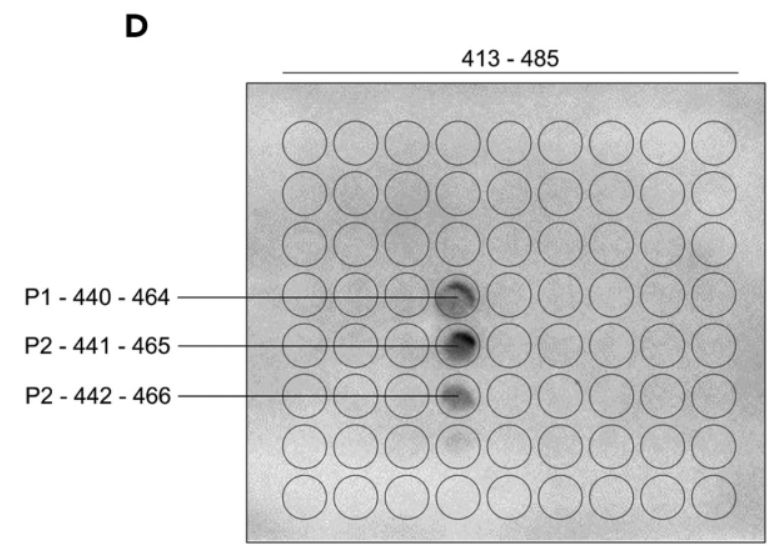

E
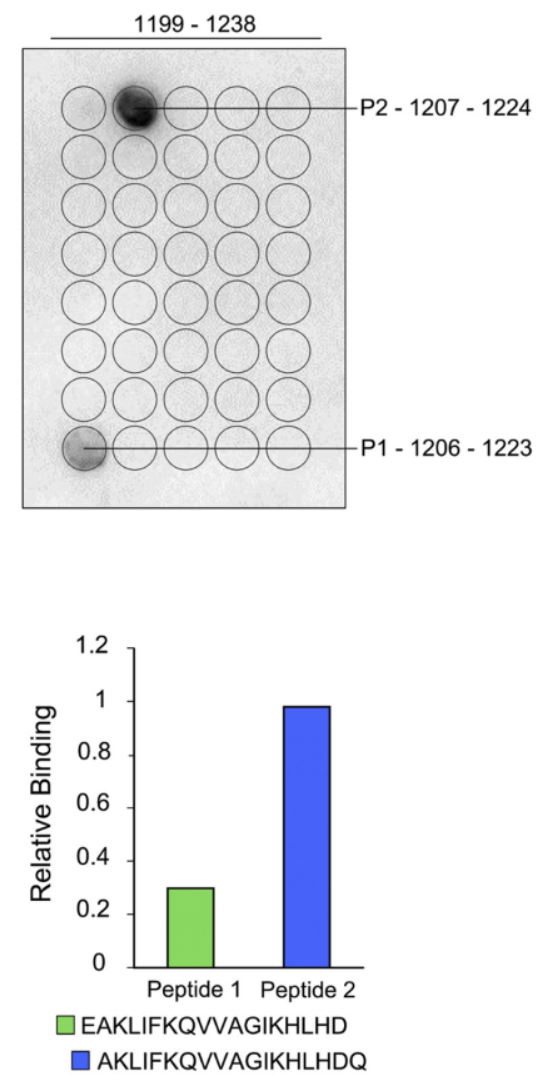

F

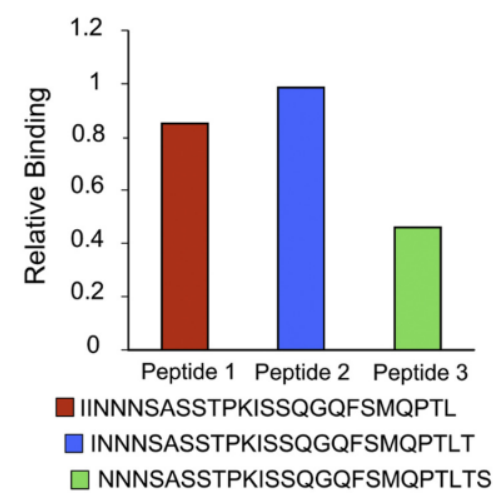

Figure 2.6: Walking peptide SPOT arrays indicate specific binding regions. SPOT arrays containing 18-aa-long printed peptides corresponding to subsequences from within the predicted interaction regions of target protein at single amino acids intervals. ( $A$ and $B$ ) Predicted interaction matrices highlight the predicted interaction regions between target ( $\mathrm{x}$ axis) and anti-target ( $\mathrm{y}$ axis). (A) The anti-Psk1/Psk1 interaction site was 
predicted to occur between residues 1209-1246 of the PSK1 protein. (B) The Pin4/antiPin4 interaction site was predicted to occur between residues 472-506 on Pin4. ( $C$ and D) SPOT arrays of predicted target binding sites and flanking regions probed with 6 xHistagged anti-target proteins followed by detection using an anti-His antibody. (C) Specific binding of the anti-Psk1 protein to the target was detected between amino acids 12041228 (D) Specific binding of the anti-Pin4 protein to the target was detected between amino acids 436-458. (E-F) Relative binding of SPOT array peptides indicates highly specific binding regions with highest relative binding.

This further supports the biological validity of our CPD method for engineering binding proteins and demonstrates the specificity of the engineered proteins. A very specific site was predicted to mediate the Psk1/anti-Psk1 interaction. This interaction region spans residues 1209-1247 in Psk1 and residues 47-69 (22 amino acids long) on the anti-Psk1 designed protein. Peptides spanning the Psk1 target region and flanking residues were probed with purified 6XHis-tagged anti-Psk1. As expected, our observations using a walking array indicate an overlap between the predicted interaction site and the actual binding sites indicated by the peptide array. Interestingly, the predicted interacting region on anti-Psk1 residues are in an area that does not have significant sequence-homology to Mmp1 (Appendix 8.1), the protein in the yeast proteome with the greatest sequence homology to anti-Psk1. Here we see how InSiPS demonstrates its ability to focus in on specific small interacting motifs that facilitate direct protein binding. Walking SPOT array analysis of the predicted Pin4 interaction region indicated a single motif which facilitates the observed binding between Pin4 and anti-Pin4 which again corresponded closely to the region predicted by InSiPS. A single region spanning residues 440-466 demonstrated binding affinity for the anti-target 
protein in the walking array. This further supports the premise that InSiPS can successfully engineer proteins that bind through short interaction motifs.

Although specific binding of both anti-Psk1 and anti-Pin4 synthetic proteins to short subsequences within the target protein was observed in the walking peptide SPOT array (Figure 2.6.C,D). We further studied the interaction between Psk1/anti-Psk1 as this peptide demonstrated the highest fitness score (Table 2.1), strongest $\mathrm{Y} 2 \mathrm{H}$ signal (Figure 2.5.), and greatest functional inhibition of the target (Figure 2.4.A,B). The walking array results showed that the highest binding intensity occurred between anti-Psk1 and a truncated version of Psk1 spanning residues 1207-1224. We probed this interaction region using a permutation array to determine which residues are most essential for binding by making single amino acid substitutions at all positions and monitoring the effect on binding affinity.

\subsubsection{Investigation of the Psk1 binding motif}

Permutation array analysis showed that amino acids 1218-1224 on Psk1 appear most essential to the interaction (Figure 2.7). This region that has 3 identical residues with the highest-scoring predicted off-target interactor Ubi4, 1219K, 1221L, and 1223D. However, the residues which have the greatest influence on the affinity between Psk1/anti-Psk1 binding are two histidines at $1220 \mathrm{H}$ and $1222 \mathrm{H}$ such that any substitution at these loci abolishes the interaction. Notably, on Ubi4, the off-target protein predicted to be the most-likely to interact with anti-Psk1, the corresponding 
residues are glutamine $(\mathrm{Q})$ and glutamate $(\mathrm{E})$. Importantly, both single amino acid substitutions, $\mathrm{H} \rightarrow \mathrm{Q}$ and a $\mathrm{H} \rightarrow \mathrm{E}$ demonstrated significantly decreased affinity for antiPsk1 protein and suggesting relatively lower affinity for the Ubi4 (Figure 2.7.D).

A

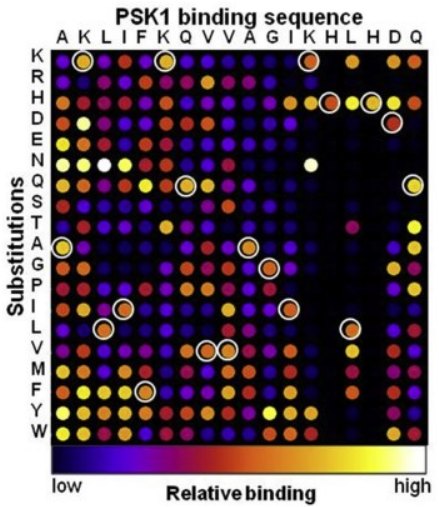

C

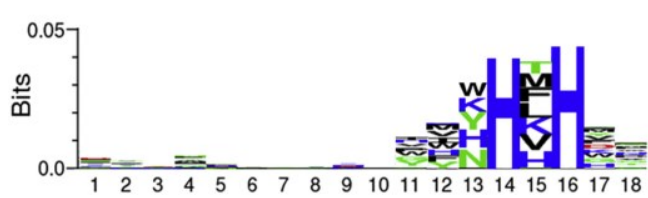

D

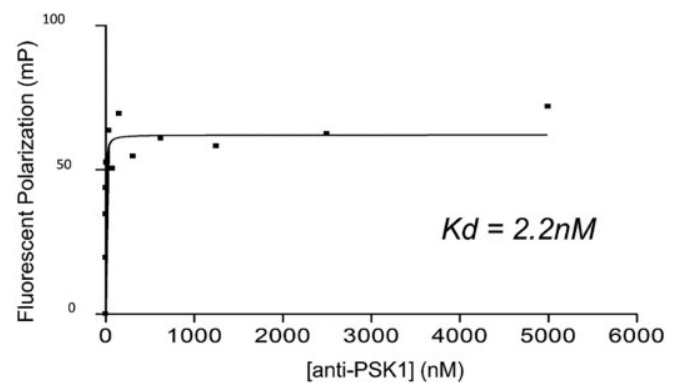

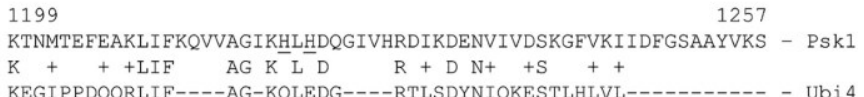

Figure 2.7: Characterization of Psk1 interaction motif and binding affinity. A) A permutation array demonstrates the relative binding affinity of anti-Psk1 to the Psk1 interaction motif (1207-1224). Peptides that correspond to the natural Psk1 protein sequence are encircled in white. Spot coloring illustrates the relative binding intensity of single amino acid substitutions at each position. B) Binding curve of the Psk1 interaction motif and anti-Psk1 produced by fluorescent polarization shows saturable binding in the nanomolar range. C) An anti-Psk1 recognition motif based on a position-scoring matrix representing the results from the permutation array in (A). D)

The specificity of our peptides is shown by the limited number of acceptable substitutions at these two key loci. Despite the sequence homology to this region on Ubi4, key residues $1222 \mathrm{H}$ and $1224 \mathrm{H}$ are expected to limit binding by anti-Psk1. 
To gauge the affinity of the anti-Psk1 protein against the predicted Psk1 binding site, fluorescent peptides corresponding to the Psk1 1207-1224 amino acid sequence were synthesized and purified for binding studies in solution. As shown in Figure 2.7.B, binding of the Psk1 (1207-1224) peptide to anti-Psk1 assumed saturable patterns with a $K d$ value of $2.2 \mathrm{nM}$. Together these results demonstrate the ability of InSiPS to engineer a small synthetic binding peptide with limited sequence homology to natural proteins that can bind with high affinity to their designated targets.

\subsection{Discussion}

We have designed and validated a unique computational tool, InSiPS, for engineering proteins that bind specified protein targets with high affinity. InSiPS offers multiple strengths over current CPD tools because of its unique methodology. By functioning as a genetic algorithm and analyzing thousands of candidate sequences over hundreds of generations, InSiPS can engineer high confidence binding proteins which limits the need for costly and time-intensive speculative laboratory trials using classical enrichment techniques. Because the algorithm analyses PPIs using patterns of primary amino acid sequences, it is not limited to working on proteins with known highresolution 3D structures/domains ${ }^{178}$ and is free of the requirement for known 3D pockets. In this way it expands our ability to tackle diverse protein targets. InSiPS also has the unique advantage of actively avoiding interactions with thousands of off-target proteins. This robust algorithm has broad applicability and could theoretically be used to 
target various proteins. These features, coupled with the wide variability of potential future applications (for example, in the areas of biomarkers and pharmacological agent development), make InSiPS a powerful CPD tool.

The efficacy of the InSiPS algorithm is demonstrated here through the successful engineering, production, and biological analysis of two SBPs, anti-Psk1 and anti-Pin4, which successfully bind to respective targets Psk1 and Pin4. Each binding protein was the manifestation of hundreds of generations of the genetic algorithm and upwards of a billion PPI predictions. Both SBPs had limited sequence-homology to endogenous yeast proteins and demonstrated relative high fitness scores $(0.465,0.380)$, predicting strong affinity for their target proteins and low affinity for all other proteins localized to the yeast cytoplasm. Consecutive biological assays verified the ability of these proteins to bind their targets both in vivo and in vitro and inhibit their natural biological functions.

Preliminary validation experiments indicated that the expression of anti-target proteins anti-Psk1 and anti-Pin-4 could functionally inhibit target proteins and produce phenotypes that resembled $\Delta p s k 1$ and $\Delta p i n 4$, respectively (Figure 2.3.A-D). $\mathrm{Y} 2 \mathrm{H}$ analysis indicated binary interactions between target and anti-target proteins in vivo (Figure 2.4) and a peptide spot array was probed in vitro to identify specific binding motifs on Psk1 and Pin4 responsible for mediating interactions. These results suggest binary interactions are occurring between anti-Psk1/Psk1 and anti-Pin4/Pin4 within predicted target regions, but further study is needed to understand binding dynamics. The antiPsk1/Psk1 interaction was further probed as it exhibited the highest fitness score, 
binding affinity, $\mathrm{Y} 2 \mathrm{H}$ signal, and functional inhibition of the target. Permutation analysis showed that a single amino acid changes to the target binding site can prevent binding of anti-target proteins. This demonstrates the ability of the genetic algorithm to "evolve" proteins that possess significant binding specificity by searching beyond natural sequence configurations. Fluorescence polarization of the Psk1 binding motif and antiPsk1 protein demonstrated a saturable pattern with a $K d$ value of $2.2 \mathrm{nM}$. The strong affinity of the anti-Psk1 protein for its target coupled with the specificity observed in the permutation analysis lends further support to the ability of the InSiPS algorithm to efficiently design proteins sequences with desired interaction properties.

Our method has furthered the field of de novo computational design of short binding polypeptides and offers promising preliminary findings, but more work is required to expand the reach and efficiency of the method. The current framework restricts InSiPS to working on annotated proteins within known PPI networks. The technology may be applicable in other systems, but this has not yet been shown. The algorithm also does not directly consider protein stability but combines aspects of known functional motifs to confer bioactivity. Because InSiPS functions in the realm of flexible linear motifs, limited structural considerations are required. Future work will examine incorporating additional structural prediction tools to predict stability and folding patterns. Another limitation may be the applicability of our approach to tackle different targets. In the current study, we started with 3 potential targets. In our first attempt we generated high scoring anti-peptides with limited off-target interactions for 
$2 / 3$ of the targets. Since each round of computation begins with a random pool of sequences, further iterations of InSiPS may identify suitable anti-peptides for Rmd1. However, with low sample numbers it remains difficult to speculate about the broad applicability of our approach to different proteins. InSiPS may also be constrained if the desired target is a member of a protein family with highly similar sequences or shares significant sequence similarity to other proteins in the cell. Lastly, the specificity of these proteins remains unclear despite being engineered to avoid off-target interactions. Only indirect and predicted evidence that our anti-target proteins avoid off-target interactions is provided in this manuscript. We did not observe significant changes in observable phenotypes when the target protein is deleted and the anti-target protein is expressed.

The current study furthers the emerging field of de novo binding protein/peptide design which strives to explore beyond natural protein sequence space and create functional high-specificity proteins that often are not found in nature. The majority of previous approaches to protein engineering have involved intelligent manipulation of naturally occurring proteins but CPD is now quickly entering a new era of de novo design. Most major advances in de novo CPD over the past few years have focused on mastering protein folding/structure prediction and using these principles to design simple novel protein structures ${ }^{179}$. For the most part, these newly designed proteins are structural with limited functionality and have simply served to lay the groundwork for future endeavors. Fundamental protein structures such as barrels ${ }^{180}$, helical bundles ${ }^{181}$, 
protein-nanomaterials ${ }^{182}$, and oligomers ${ }^{183}$, have been developed and may eventually be used for variety of future applications as we delve further into sequence space and take advantage of the scalability and specificity of polypeptides. However, at this point, very few novel functional polypeptides have been developed computationally de novo.

Proteins/peptides with short highly specific binding motifs, like the ones developed in this study could be developed to function as aptamers, research tools, biomarkers, pharmacological agents or more. Peptide aptamers continue to be developed for a variety of industrial ${ }^{138}$ and medicinal ${ }^{184}$ applications but the field of using proteins as pharmacological agents has expanded significantly over the past 5-7 years. Peptide therapeutics can be tailored to maximize compatibility, stability, and potency with relative ease and there are currently over 60 available FDA-approved peptide drugs with another $500+$ progressing through the development stage ${ }^{153,185}$. Other potential applications of this methodology include the development of biosensors for previously unreachable biomarkers or the intelligent design of peptide aptamers. Certain small protein targets such as the medically relevant angiotensinogenase renin have proven difficult to probe with traditional approaches but can be detected using peptide-based aptamers developed using cDNA display techniques ${ }^{186}$. Peptides designed to function as therapeutics can outperform relatively larger ( 150kDa) antibodies in terms of bioavailability, tumor penetration and production efficiency and have been used to develop binding assays, cancer therapy, drug delivery and in vivo imaging ${ }^{187}$. Peptides, which were previously thought of as ineffective pharmacologically 
due to poor delivery mechanisms and rapid degradation/clearance ${ }^{151}$, are changing preconceptions as synthetic peptide production decreases in cost, new delivery systems are developed, and biological production using vectors such as viruses or genetic manipulation via CRISPR-Cas9 become more realistic possibilities.

\subsubsection{Summary}

To summarize, we have presented a unique CPD tool that employs a massivelyparallel genetic algorithm and PPI-prediction tool to engineer binding peptides against protein targets. We demonstrated that two synthetic proteins engineered by InSiPS can bind endogenous yeast proteins at predicted motifs and inhibit functionality. Further work will examine if the technique can work in other species and explore the range of potential targets. We invite those interested in using InSiPS to contact the corresponding author. By combining constructive techniques such as InSiPS with modeling technologies, future techniques will aim to develop highly stable, specific binding proteins for a range of applications.

\subsubsection{Limitations of the Study}

Limitations of the Study Our method has furthered the field of de novo computational design of short binding polypeptides and offers promising preliminary findings, but more work is required to expand the reach and efficiency of the method. The current framework restricts InSiPS to working on annotated proteins within known PPI networks. The technology may be applicable in other systems, but this has not yet 
been shown. The algorithm also does not directly consider protein stability but combines aspects of known functional motifs to confer bioactivity. Because InSiPS functions in the realm of flexible linear motifs, limited structural considerations are required. Future work will examine incorporating additional structural prediction tools to predict stability and folding patterns. Another limitation may be the applicability of our approach to tackle different targets. In the current study, we started with three potential targets. In our first attempt, we generated high-scoring anti-peptides with limited off-target interactions for two of three targets. As each round of computation begins with a random pool of sequences, further iterations of InSiPS may identify suitable anti-peptides for Rmd1. However, with low sample numbers it remains difficult to speculate about the broad applicability of our approach to different proteins. InSiPS may also be constrained if the desired target is a member of a protein family with highly similar sequences or shares significant sequence similarity with other proteins in the cell. Last, the specificity of these proteins remains unclear despite being engineered to avoid off-target interactions. Only indirect and predicted evidence that our anti-target proteins avoid off-target interactions is provided in this article. We did not observe significant changes in observable phenotypes when the target protein is deleted and the anti-target protein is expressed (Figures 2.4.A-B) and show specificity through the binding observed in the SPOT arrays (Figures 2.6 and 2.7). 


\section{Chapter 3: PAS kinase Psk1 influences non homologous end- joining fidelity}

\subsection{Abstract}

Non-homologous end-joining (NHEJ) is a highly conserved pathway responsible for repairing double-strand breaks (DSBs). Despite extensive study, novel repair factors continue to be identified. Here we report on novel NHEJ factor PSK1 which when deleted sensitizes cells to numerous DNA damaging agents and greatly decreases the efficiency of NHEJ repair on both plasmid and chromosomal DSBs. Functional genetic analysis identified a relationship between PSK1 and NHEJ repair factor RAD27. The overexpression of RAD27 in a $\triangle p s k 1$ mutant background suppresses deficits caused by the loss of PSK1 function. This non-reciprocal relationship (PSK1 does not rescue rad27A phenotype) suggests that PSK1 may function upstream of RAD27 and work to promote RAD27 activity in NHEJ. This identification of a novel mechanism expands our current understanding of the NHEJ pathway and introduce new players and novel functional relationships.

\subsection{Introduction}

Ineffective repair of DNA double strand breaks (DSBs) can result in severe mutations, senescence or damage-induced cell death. To avoid such outcomes, eukaryotic cells initiate a global cellular response to DSBs encompassing a highly 
interconnected network responsible for detection, cell cycle arrest, activation of the appropriate repair pathway, and eventually, return to the cell cycle ${ }^{107}$. DSBs arise regularly during all cell cycle stages for a variety of reasons. Sources of these breaks may be endogenous including DNA-damaging metabolic byproducts, collapsed replication forks, and nuclease activity or exogenous including IR or UV radiation and DNA damaging chemicals ${ }^{188}$. Given the unpredictable nature of DBSs, the cellular response must be highly adaptable and possess sufficient plasticity to function regardless of the current cellular environment, cell cycle stage, level of chromatin packaging, template availability and biochemical variation in the break structure. The inherent plasticity required to mount an effective response to DSBs helps to explain why novel repair factors continue to be discovered despite a general understanding of the core repair pathways.

Two archetypal pathways function to process DSBs: i) homologousrecombination $(\mathrm{HR})$ repair which functions when a homologous sequence is available to facilitate accurate template-based repair and ii) the non-homologous end joining (NHEJ) repair pathway which processes damaged DNA strands prior to directly-ligating broken ends through a relatively more error-prone mechanism. HR is the primary repair pathway employed by yeast while NHEJ dominates in mammalian systems ${ }^{189}$. Yeast has long served as an effective model for both pathways and provided numerous insights into the molecular mechanisms underlying DSB repair ${ }^{124,131,190,191}$. In haploid yeast, HR dominates from S-phase until division when a homologous chromosome is present 
while NHEJ is the preferred mechanism during $\mathrm{G} 1$ when a template sequence is generally not available ${ }^{192}$. Each pathway employs a unique set of proteins to facilitate repair and the highly amenable yeast model facilitates studies of both.

HR begins when a significant 5'to $3^{\prime}$ end-resection occurs at the broken ends. Rad51, the strand exchange protein, is loaded on to single-stranded DNA to produce $3^{\prime}$ nucleoprotein filaments that facilitate a homology search then invades homologous regions and utilize Rad52 and Rad54 to form a heteroduplex displacement loop (D-loop) 193. DNA is then synthesized using the $3^{\prime}$-end as a primer and the homologous regions as a template and the structure is resolved by one of three resolution mechanisms ${ }^{194}$. In yeast, NHEJ acts as an alternative pathway which directly ligates broken ends following minimal resection when a homologous template is unavailable, essential HR repair factors are absent, or when HR has failed.

NHEJ can occur at all stages of the cell cycle and is initiated when the YKU heterodimer complex (Yku70/Yku80) attaches to broken ends which prevents further resection and destines the break for end-joining ${ }^{107}$. Binding of YKU recruits' core NHEJ machinery including the MRX (Mre11-Rad50-Xrs2) complex which aligns broken ends and facilitates preliminary end processing, the multifunction Nej1 protein, and the DNA ligase IV complex (Dnl4/Lif4). In addition to this "core" machinery, many other factors are important for effective NHEJ. For example, Pol4, an error-prone member of the Polymerase X family, functions to fill small gaps and can function when only limited sequence homology is present ${ }^{128}$. Another NHEJ repair factor is the Rad27 nuclease. 
There is some ambiguity in relation to Rad27s role and importance in NHEJ as it has been shown to be important for break structures that produce $5^{\prime}$ flaps during repair while others report that it is unnecessary when repairing complex break structures $118,195,196$. Knowledge gaps remain in understanding the entire complement of factors that contribute to yeast NHEJ, particularly in the area of signaling pathways.

Communication during NHEJ relies on a multitude of kinases to amplify the damage signal and facilitate checkpoint activation and an appropriate repair response. Mec1 and Tel1 kinases are initially recruited to DSBs and respond by phosphorylating multiple targets including the Rad53 and Chk1 checkpoint kinases and histone $\mathrm{H} 2 \mathrm{~A}^{94}$. Mec1, the human ATR homolog and its partner Ddc2 function together in association with Tel1, the ATM homolog in facilitating downstream DSB checkpoint activation ${ }^{96,197}$.

PAS kinases, originally recognized as a group of nutrient-sensing kinases, attracted interest as the only metazoan protein to possess both a regulatory PAS domain as well a functional threonine/serine kinase domain ${ }^{198}$. PAS domains, present in species ranging from simple bacteria to complex eukaryotes, function as nutrientsensing modules that respond to a broad range of nutritional conditions/stimuli and can activate signaling pathways to adjust metabolism as needed through regulation of arange of downstream processes ${ }^{198,199}$. PAS kinase functions are similar to other medically relevant nutrient sensing protein kinases such as AMPK and mTOR. In S. cerevisiae, PAS kinases Psk1 and Psk2 respond to nutrient or cell-integrity stress by increasing carbohydrate metabolism at the expense of storage through phosphorylation 
of Ugp1 ${ }^{200}$. Psk1 and Psk2 are partially-redundant PAS kinase homologs that likely arose through whole-genome duplication in a yeast ancestor and both respond to cellular stress but demonstrate unique functional roles ranging from glucose partitioning, cellular homeostasis, response to oxidative stress and translation ${ }^{201-203}$. Of note, PSK1 has been shown to be important for protecting cells against oxidative stress, particularly through the induction of superoxide-dismutase 1 (SOD1) expression ${ }^{202}$.

The accumulation of reactive oxygen species (ROS) can lead to DNA damage and inversely, DNA damage can lead to the accumulation of ROS ${ }^{204}$. Pathways responsible for DNA damage repair (DDR) are associated with those that respond to oxidative stress. PSK1, a dynamic kinase that is activated in responses to oxidative stress, shares significant homology with two human proteins, PIM1 and PASK. Protein-protein interaction (PPI) prediction analysis using PIPE suggested functional interactions with known NHEJ proteins. These predictions coupled with a the previously reported role of Psk1 in the cellular response to oxidative stress helped identify the PSK1 gene as a potential participant factor in the non-homologous end joining pathway (NHEJ). Given the considerable interest in identifying novel participants in NHEJ, we investigated a potential role for PSK1 within the pathway and concluded that Psk1 does influence both the efficiency and fidelity of homology-free double-strand break (DSB) repair in yeast. Functional genetic analysis performed herein indicates that the influence of PSK1 on NHEJ is related at least in part to the role of endonuclease RAD27. The loss of PSK1 sensitizes cells to DNA damaging agents but the overexpression of RAD27 can suppress 
this phenotype. Together our results identify the PSK1 PAS kinase gene as a potential DSB repair factor and suggest a functional association with RAD27.

\subsection{Materials and Methods}

\subsubsection{Yeast strains and plasmids}

Strains used in initial plasmid repair and genetic interaction analysis are derived from a BY4741 background (MATa orf $\Delta:: K a n M X 4$ his3 $\Delta$ leu2 $\Delta$ met15 $\Delta$ ura3 $\Delta)^{205}$. Loss of function mutants for various single and double mutants were created in Y7092 (MAT $\alpha$

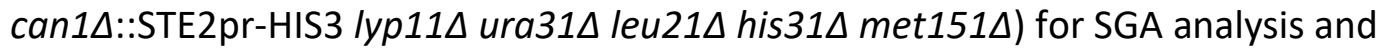
JKM139 (MATa $h m r \Delta:: A D E 1 ~ h m l \Delta:: A D E 1$ ade1-100 leu2-3,112 lys5 trp1::hisG ura3-52 ade3::GAL-HO) for chromosomal break and repair fidelity analysis, through a PCR-based transformation and replacement of targeted genes by either nourseothricin $\left(\mathrm{Nat}^{\mathrm{R}}\right)$ or hygromycin $\left(\mathrm{Hyg}^{\mathrm{R}}\right)$ resistance casettes. Plasmid repair assays were carried out using p416. pMV1328 carrying LEU2 and $\operatorname{Kan}^{R}$ markers was used for the repair fidelity assay ${ }^{124}$. Derivatives of pGEH expression plasmids were used for various genetic analyses ${ }^{206}$. Plasmids were propagated in $\mathrm{DH} 5 \alpha$ and isolated via ThermoFisher plasmid mini-prep kits.

\subsubsection{Spot test analysis of sensitivity to DNA damaging agents}

Various strains were grown to saturation in $5 \mathrm{~mL}$ of YPD or $5 \mathrm{~mL}$ of -ura medium for 2 days. Saturated cultures were serially diluted $10^{-1}-10^{-4}$ and $15 \mu \mathrm{l}$ of each dilution 
was spotted on solid media. For general conditional sensitivity analysis, cells were plated on YPD, YPD+0.05\% methylmethane sulfonate (MMS), YPD+0.2M hydroxyurea, or YPD exposed to ultraviolet radiation for $30 \mathrm{sec}$. Sensitivity analysis for assays involving overexpression plasmids were plated on $\mathrm{YP}+2 \%$ galactose. The viability of each strain was assessed after 2-3 days of growth at $30^{\circ} \mathrm{C}$. Results are presented as photographs or the results of multiple visually scored trials in which the growth rate is scored 1-4 based on comparative growth rates $(4=$ comparable to WT growth rate, $1=$ very minimal growth) and average results from three trials +/- standard deviation are presented.

\subsubsection{Plasmid repair assay}

p416 was digested at a single Xbal restriction site which lacks homology to yeast chromosomal DNA and required NHEJ repair for proliferation. Parallel transformation of digested and intact plasmid was carried out in WT and mutant strain. After three days of growth, colonies were counted and the ratio of linear to circular colonies, normalized to WT ratio, was used to assess the repair efficiency 207,208 .

\subsubsection{Chromosomal break assay}

Single and double mutants were created in the JKM139 background for analysis. In this background, growth in the presence of galactose induces expression of a GAL1 regulated $\mathrm{HO}$ endonuclease (expression suppressed by glucose). HO repeatedly induces DSBs at the MAT locus. Homologous regions related to mate-type switching are removed in this background and the severed chromosome must be repaired via NHEJ. 
WT, mutants and mutants carrying overexpression plasmids were grown in synthetic complete or selective medium to saturation and were then serially diluted to concentrations of $10^{-1}$ to $10^{-4} .100 \mu \mathrm{L}$ of the $10^{-2}$ dilution were plated on YP-Galactose as experimental plates and $100 \mu \mathrm{L}$ of the $10^{-4}$ dilution was plated on YPD control plates. Colonies were counted after 2 days of incubation at $30^{\circ} \mathrm{C}$. Results are presented as the ratio of experimental/control counts normalized to the JKM139 (WT) control ratio. Results are presented as the average result of four trials +/- standard deviation.

\subsubsection{Genetic interaction analysis}

Synthetic genetic array (SGA) analysis was performed as in Tong et al, $2006{ }^{209}$, and phenotypic suppression analysis (PSA) was performed as in Sopko et al, $2006{ }^{206}$. Strains carrying deletion or overexpression of PSK1 in the Y7092 background were crossed to a DNA Damage Array (DDA) containing deletion strains of 384 genes associated with DNA repair, DNA damage response, cell cycle, and chromatin remodeling. The resultant strains were then grown on medium containing $30 \mathrm{mM} \mathrm{HU}$ and $1.6 \mu \mathrm{g} / \mathrm{mL}$ PHLM to test for conditional genetic interactions. Results were visually scored. Each experiment was repeated three times.

\subsubsection{Repair fidelity assay}

Plasmid pMV1328 was digested by Pstl within its KanMX6 marker. Digested and circular plasmids were transformed into mutant and WT strains in JKM139 background. Cells were grown on synthetic medium lacking leucine for 2-3 days. To test the accuracy 
of repair, 50-100 transformants (colonies) were grown on YPD medium containing G418 $(200 \mu \mathrm{g} / \mathrm{mL})$. Growth on $\mathrm{G} 418$ represented accurate repair ${ }^{132}$. Repair fidelity is represented as the ratio of viable cells over total transformants transferred and normalized to the WT ratio.

\subsubsection{Growth curve analysis}

The WT, single mutant strains, and mutant strains with overexpression plasmids, were grown to saturation in $5 \mathrm{~mL}$ of SC medium (-ura for plasmid strains) for approximately 48 hours at $30^{\circ} \mathrm{C}$ with shaking at $200 \mathrm{rpm}$. The cells were washed twice and were resuspended in $5 \mathrm{~mL}$ of distilled water. The cell culture density was measured by determining the $\mathrm{OD}_{600}$ of 1:10 dilutions of the resuspended culture and the cell density was extrapolated using a standard curve of yeast spectrophotometric data. The suspensions were standardized by diluting each culture to the lowest density observed, so that all suspensions had the same cell density. $20 \mu \mathrm{L}$ of the diluted culture was transferred to a microplate containing $180 \mu \mathrm{L}$ of YP-Gal and YP-Gal + MMS (0.005\%) and grown for 18 hours at $30^{\circ} \mathrm{C}$. The $\mathrm{OD}_{600}$ was taken every 30 minutes, with orbital shaking for 30 seconds prior to every reading, using a BioTek FL600 microplate reader at

$30^{\circ} \mathrm{C}$. All samples were analyzed in triplicate and presented as the mean $+/-\mathrm{SD}$ of $O D_{600}$ readings. Three independent trials were performed for all analyses. 


\subsection{Results}

\subsubsection{Identification of Psk1 as a novel NHEJ repair factor}

The S.cerevisiae PSK1 gene codes for a kinase with multiple roles in signaling to promote cell survival in response to changing environments. Its highly-conserved PAS threonine/serine kinase domain has strong sequence similarity to the human PIM1 proto-oncogene kinase domain as demonstrated in Figure 3.1.A. PIM1 was identified as one of several possible novel DSB repair factors in a screen for proteins that were predicted to physically interact with important NHEJ repair proteins ${ }^{66}$. PIM1 interactors were enriched in processes such as: i) Cellular response to DNA damage stimulus GO:0006974 $-p=5.3 \mathrm{E}^{-10}$, ii) Signal transduction involved in DNA damage checkpoint GO:0072422 $p=3.5 E^{-9}$, and iii) Regulation of DNA repair $p=2.7 E^{-6}$. Given the enriched predicted interconnectivity between PIM1 and multiple NHEJ repair factors, we hypothesized that the homologous yeast Psk1 protein may also play a role in DSB repair. If the conserved PAS kinase domain does in fact play a role in yeast DNA repair, it may have potential implications for human health. To determine if yeast Psk1 influences DSB repair, particularly via the medically important NHEJ pathway, we subjected the deletion mutant to a complement of preliminary DNA repair assays.

Three classic assays were employed initially to assess if the loss of PSK1 affects DSB repair efficiency. These included a plasmid repair efficiency assay and chromosomal break assay engineered to restrict repair to the NHEJ pathway, and conditional 
sensitivity analysis using a variety of DNA-damaging agents (Figure 3.1). The conditional sensitivity assay is used to indicate if the loss of PSK1 results in a change in ability to respond to various forms of widespread DNA damage stress where sensitivity may indicate a role in DNA repair pathways or the DNA damage response. The plasmid repair and chromosomal break assays, however, are used to screen for any influence of the gene on the functioning of the NHEJ pathway.

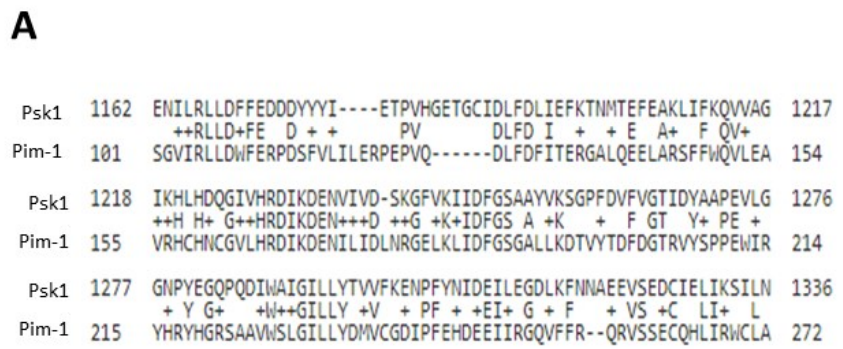

B

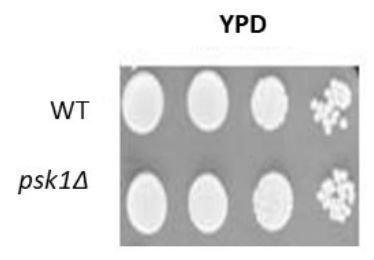

UV

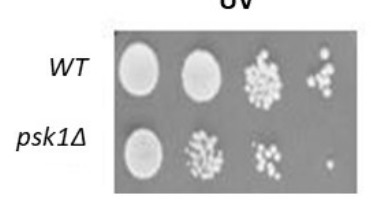

MMS

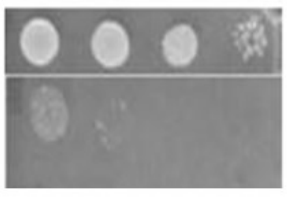

Hydroxyurea

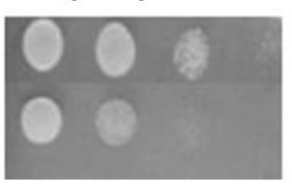

C

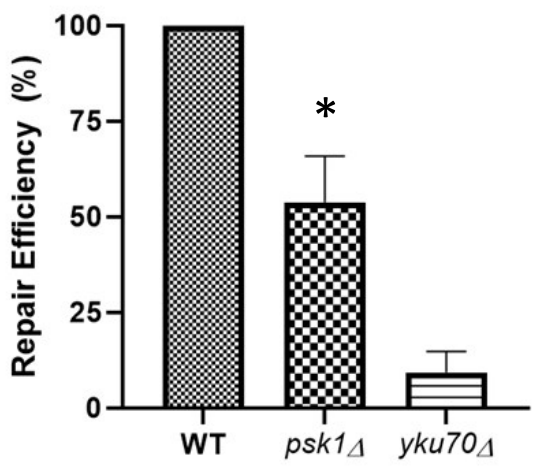

D

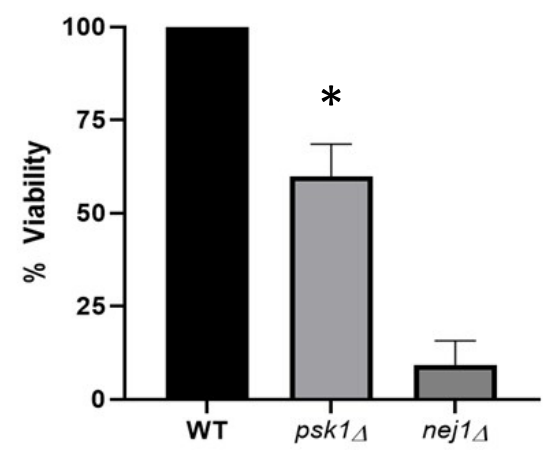

Figure 3.1: Analyzing the influence of PSK1 on DNA repair. (A) Yeast Psk1 has significant protein sequence homology with a region of human PIM1 (BlastP alignment: Expect $=4 \mathrm{e}^{-}$ $37,34 \%$ identity, $54 \%$ similarity). (B) Conditional-sensitivity analysis indicates that PSK1 mutants are sensitive to multiple DNA-damaging agents. Cultures grown to saturation were serially diluted from $10^{-1}-10^{-4}$ and spotted on YPD, YPD+0.05\% methylmethane sulfonate (MMS), YPD+0.2M hydroxyurea, or YPD exposed to ultraviolet radiation for 30 sec. (C) Plasmid repair assay using Xbal-linearized p416 shows that psk1 $\Delta$ mutants are $46 \%$ less efficient at NHEJ repair. (D) The chromosomal break assay indicates that the 
loss of PSK1 causes a significant reduction in the ability to repair severed chromosomes (60\% compared to WT). Statistical analysis in C-D performed with a standard two-tailed T-test, significance is indicated by $p$-values $<0.05$ using a minimum of three replicates.

Together, the findings displayed in Figure 3.1 suggest a role for Psk1 in the response to DNA damage, specifically through the NHEJ pathway. Psk1 mutants showed moderate sensitivity to UV light stress and hydroxyurea but were highly sensitive to MMS (Figure 3.1.B). Combined, these sensitivities support a role for PSK1 in the ability of cells to respond to multiple forms of DNA damage stress but do not indicate a specific pathway. The reduction of repair efficiency of the psk1 $1 \Delta$ mutants in both the plasmidbased ( $46 \%$ reduction) and chromosomal break assay ( $40 \%$ reduction) on the other hand provide evidence that PSK1 influences the NHEJ pathway. Because of this initial evidence that PSK1 is in fact a novel NHEJ repair factor, a series of genetic interaction (GI) screens and functional genetic analyses were subsequently employed to narrow-in on the specific method(s) by which PSK1 may influence this multifaceted repair pathway.

\subsubsection{Analyzing the conditional genetic interaction profile of PSK1 under}

\section{DNA damage stress}

Two genetic screening approaches were used to study PSK1 function in relation to DNA repair, synthetic genetic array (SGA) and phenotypic suppression analysis (PSA). Both approaches were conducted using a DNA damage array (DDA), an array of 380 single mutants that have functions related to DNA repair, the DNA damage response 
and chromatin remodeling and other related processes. SGA analysis involves the systematic production of double mutants via replica pinning events that eventually select for cells lacking your query gene and a gene from the array ${ }^{209}$. By mating PSK1 with the DDA and performing colony-size analysis, we screened for Gls that may link PSK1 to specific processes in DNA repair.

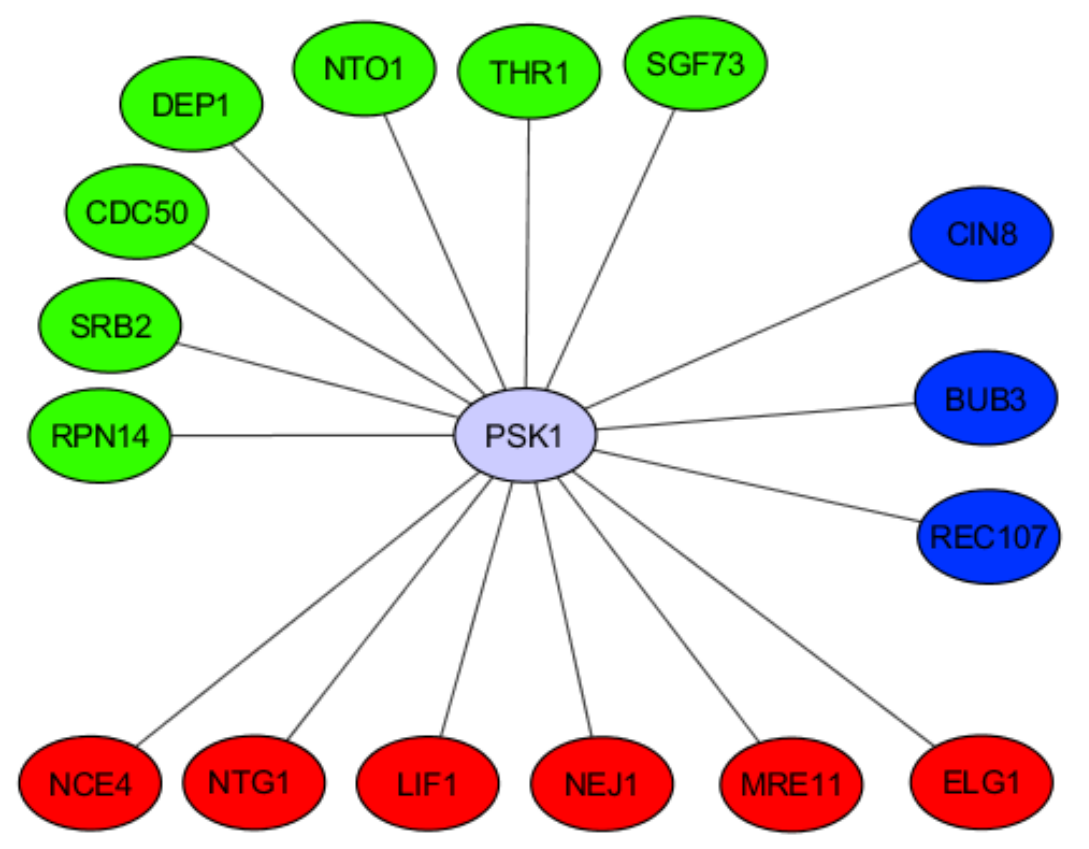

Figure 3.2: A genetic interaction map produced by SGA analysis of PSK1. Genes displayed here had a "sick" phenotype when crossed with PSK1 indicating a negative genetic interaction. Interacting analysis indicated functional associations with genes related mitotic cell cycle progression (blue), DNA repair (red) and various other processes (green). 
Table 3.1: Summary of PSK1 genetic interactors from SGA analysis and shown in Figure 3.2. Interaction analysis indicated functional associations with genes related to DNA repair (red), mitotic cell cycle progression (blue), and various other processes (green).

\begin{tabular}{|c|c|}
\hline Gene & Overview of function \\
\hline NCE4 & $\begin{array}{l}\text { Subunit of the RecQ (Sgs1p) - Topo III (Top3p) complex; stimulates } \\
\text { superhelical relaxing, DNA catenation/decatenation and ssDNA binding } \\
\text { activities of Top3p; involved in response to DNA damage; }\end{array}$ \\
\hline NTG1 & $\begin{array}{l}\text { DNA N-glycosylase and apurinic/apyrimidinic (AP) lyase; involved in base } \\
\text { excision repair; acts in both nucleus and mitochondrion; }\end{array}$ \\
\hline LIF1 & $\begin{array}{l}\text { Component of the DNA ligase IV complex; this complex mediates } \\
\text { nonhomologous end joining in DNA double-strand break repair; physically } \\
\text { interacts with DnI4p and Nej1p; homologous to mammalian XRCC4 protein }\end{array}$ \\
\hline NEJ1 & $\begin{array}{l}\text { Protein involved in regulation of nonhomologous end joining; interacts with } \\
\text { DNA ligase IV components DnI4p and Lif1p; repressed by MAT } \\
\text { heterozygosity; regulates cellular distribution of Lif1p }\end{array}$ \\
\hline MRE11 & $\begin{array}{l}\text { Nuclease subunit of the MRX complex with Rad50p and Xrs2p; complex } \\
\text { functions in repair of DNA double-strand breaks and in telomere stability; }\end{array}$ \\
\hline ELG1 & $\begin{array}{l}\text { Subunit of an alternative replication factor C complex; important for DNA } \\
\text { replication and genome integrity; suppresses spontaneous DNA damage; } \\
\text { involved in homologous recombination-mediated repair }\end{array}$ \\
\hline BUB3 & $\begin{array}{l}\text { Kinetochore checkpoint WD40 repeat protein; localizes to kinetochores } \\
\text { during prophase and metaphase, delays anaphase in the presence of } \\
\text { unattached kinetochores; forms complexes with Mad1p-Bub1p }\end{array}$ \\
\hline REC107 & $\begin{array}{l}\text { Protein involved in early stages of meiotic recombination; involved in } \\
\text { coordination between the initiation of recombination and the first division } \\
\text { of meiosis; part of a complex (Rec107p-Mei } 4 p-\operatorname{Rec} 114 p \text { ) }\end{array}$ \\
\hline CIN8 & $\begin{array}{l}\text { Kinesin motor protein; involved in mitotic spindle assembly and } \\
\text { chromosome segregation }\end{array}$ \\
\hline DEP1 & Component of the Rpd3L histone deacetylase complex; \\
\hline CDC50 & $\begin{array}{l}\text { Endosomal protein that interacts with phospholipid flippase Drs2p; } \\
\text { interaction with Cdc50p is essential for Drs2p catalytic activity; }\end{array}$ \\
\hline RPN14 & Evolutionarily conserved 19S regulatory particle assembly-chaperone; \\
\hline SGF73 & $\begin{array}{l}\text { Subunit of DUBm module of SAGA and SLIK; has roles in anchoring } \\
\text { deubiquitination module (DUBm) into SAGA and SLIK complexes, }\end{array}$ \\
\hline SRB2 & Subunit of the RNA polymerase II mediator complex; \\
\hline THR1 & Homoserine kinase; conserved protein required for threonine biosynthesis; \\
\hline NTO1 & $\begin{array}{l}\text { Subunit of the NuA3 histone acetyltransferase complex; this complex } \\
\text { acetylates histone H3; }\end{array}$ \\
\hline
\end{tabular}


SGA analysis is a powerful functional genetic approach that generally identifies genes involved in distinct functionally overlapping pathways. In other words, for a negative $\mathrm{GI}$ to be detected in a double-mutant, each of the genes involved likely plays a unique role in redundant pathways that produce similar outcomes ${ }^{210}$. Psk1 was observed to interact with several key NHEJ genes including LIF1, MRE11, and NEJ1 (Figure 3.2 and Table 3.1). Additionally, interactions with genes known to function in other repair pathways such as base excision repair (BER) factor NTG1, an apurinic/apyrimidinic lyase, and HR repair factor ELG1 were seen 211,212 .

To further probe the role of PSK1 within NHEJ, we employed a complementary genetic screening technique to determine if the overexpression of PSK1 can phenotypically compensate for deletions that sensitize cells to DNA damaging agents. PSA analysis of PSK1 showed phenotypic suppression of genes associated with cell cycle progression, chromatin remodeling and DSB repair among others (Figure 3.3). 


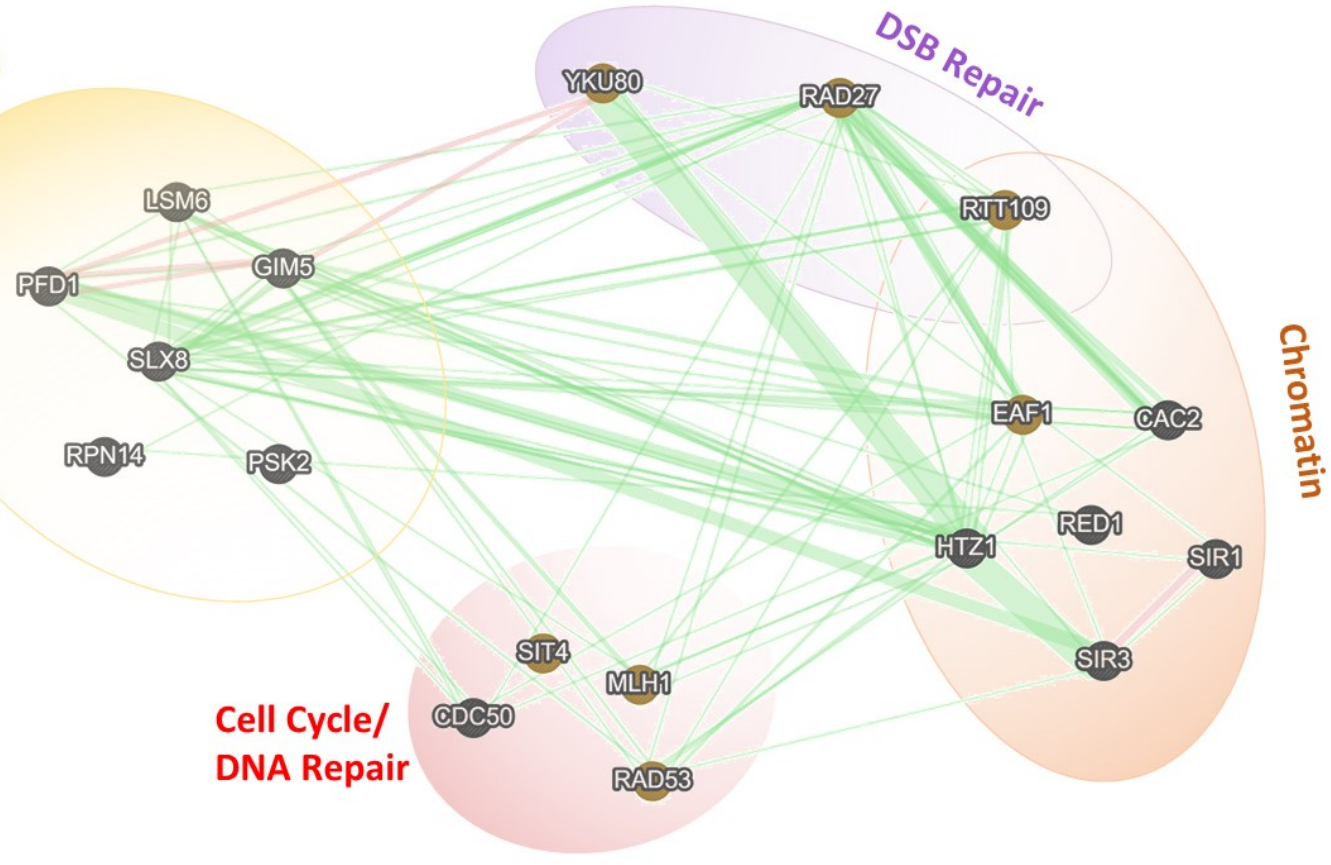

Figure 3.3: Results from PSA analysis indicate interactions between PSK1 and genes related to cell cycle progression, chromatin remodeling, DSB repair, and other processes. Only suppression interactions capable of complete/near-complete recovery are presented in this figure. Edges indicated previously reported GIs (green) and PPIs (pink) as reported in GeneMania. Nodes with yellow background are annotated with the GO Term: DNA Repair.

Phenotypic (genetic) suppression is observed when a functional defect caused by a loss of gene is rescued by the overexpression of another gene. The epistatic interactions identified by PSA often indicate genes that function within the same complex or pathway, potentially indicating directionality within said pathway. However, in certain cases, they may also indicate alternative or compensating pathways ${ }^{213}$. Amongst the interactors identified in the PSK1 PSA analysis were genes that function in cell cycle regulation and/or DNA repair including SIT4, a serine-threonine phosphatase that functions in repair and G1-S transition, mismatch repair factor $M L H 1$, and $C D C 50$, a 
gene also detected in SGA analysis which primarily functions as a phospholipid flippase but also has a putative role in promoting G1-S transition. Notably, a functional relationship with key DNA damage checkpoint RAD53 was detected. PSK1 was also seen to compensate for its paralog PSK2 as well as histone variant HTZ1 (H2A.Z) and histone acetyltransferase $R T T 109$ which plays a role in DSB repair during S-phase ${ }^{208,214}$.

Importantly, the overexpression of PSK1 rescued multiple mutant strains directly involved in NHEJ repair. Given the evidence to suggest a role for PSK1 in NHEJ and the importance of the NHEJ repair pathway, these interactions were of significant interest. Specifically, genetic compensation was shown for NHEJ repair genes RTT109, YKU80, and $R A D 27$. RAD27 was identified as being of particular interest as it was the only NHEJ repair gene whose absence was fully compensated for by PSK1 overexpression when challenged by both hydroxyurea and phleomycin. To further probe the genetic interactions of PSK1, a reverse PSA analysis was performed on several genes of interest related to those identified in the previous genetic screens and the predicted interaction network of human PIM1. In this assay, selected candidate genes were overexpressed in a psk1 $\Delta$ background to identify cases of functional compensation (Figure 3-4) under three damage stress conditions, UV light, MMS, and hydroxyurea. 
Table 3.2: Genetic compensation analysis of the overexpression of various genes of interest in a psk1 $1 \Delta$ background. Constructs were exposed to conditions of UV light exposure (30 sec), $0.04 \% \mathrm{v} / \mathrm{v} \mathrm{MMS}$, or $70 \mathrm{mM} \mathrm{HU}$. Cultures were grown to saturation and then serially diluted and spot-tested on YPGal or YPGal + Drug (or UV). Visual analysis of three repeats assessed growth relative to WT. Constructs that had fitness above the PSK1 mutant are denoted as "+" while lower fitness is denoted as "-".

\begin{tabular}{llccc}
$\begin{array}{l}\text { Overexpressed } \\
\text { gene }\end{array}$ & Function & UV & MMS & HU \\
\hline RAD50 & MRX complex & + & - & - \\
MRE11 & MRX complex -3'-5' nuclease & + & + & + \\
SWI6 & G1/S transition & + & - & + \\
RAD53 & Damage response kinase, activates Dun1 & - & - & - \\
DUN1 & Checkpoint kinase required for G2/M arrest & - & + & + \\
MRC1 & S-phase checkpoint protein & - & - & + \\
RAD27 & 5'-3' exonuclease, disrupted role in NHEJ $^{\prime}$ & + & + & + \\
\hline
\end{tabular}

The reverse complementarity assay demonstrated that the overexpression of several candidate genes functionally compensated for the loss of PSK1 and suggesting a robust role for PSK1 in various aspects of DNA repair (Table 3.2 and Appendix 8.2). Particularly, the overexpression of either MRE11 or RAD27 nucleases suppressed the PSK1 mutant phenotype in all three conditions tested. The results of these functional genetic analyses indicate that PSK1 may work to influence nuclease activity. The functional relationship with $R A D 27$ was uniquely interesting as $R A D 27$ has an ambiguous role in NHEJ but repeatedly demonstrated a strong functional relationship with PSK1 activity. The originality of this finding coupled with the connection between these two novel repair factors warranted further study into the influence of PSK1 and RAD27 on NHEJ repair dynamics. 


\subsubsection{Studying the functional relationship between RAD27 and PSK}

To study the relationship between these two genes, additional directed

functional genetic tests were performed. The results in Figure 3.4 indicate that overexpression of RAD27 compensates for the loss of PSK1 but inversely, excess Psk1 protein does not compensate for deletion of the RAD27 exonuclease (Figure 3.4.A). Additionally, double mutant phenotypic analyses indicate that PSK1 and RAD27 appear to function in the same pathway. Double deletion mutants do not further reduce the efficiency of DSB repair beyond the deficits seen in either single mutant in both the plasmid repair assay and the chromosomal break assay (Figure 3.4.C,D).
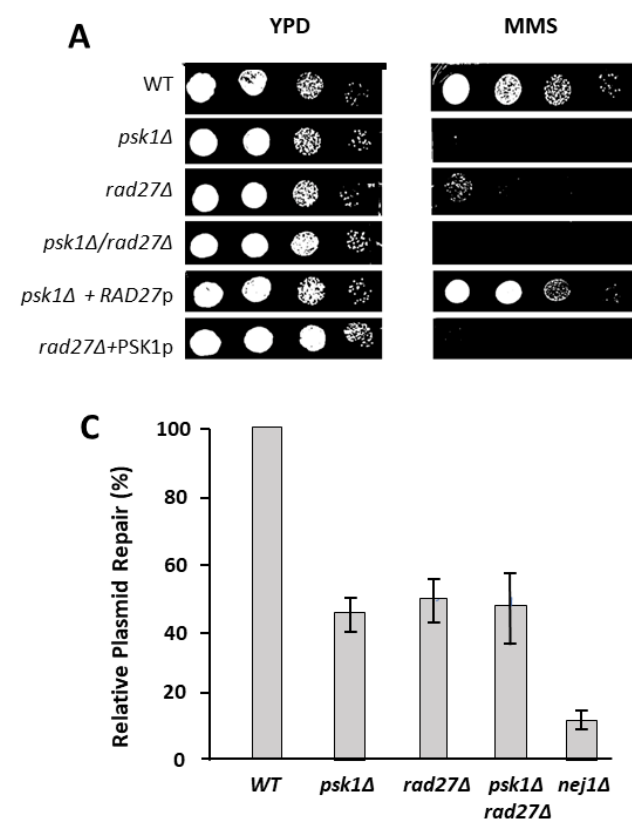

B

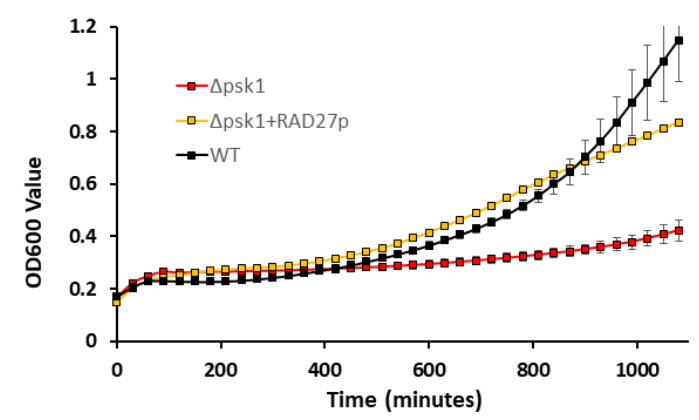

D

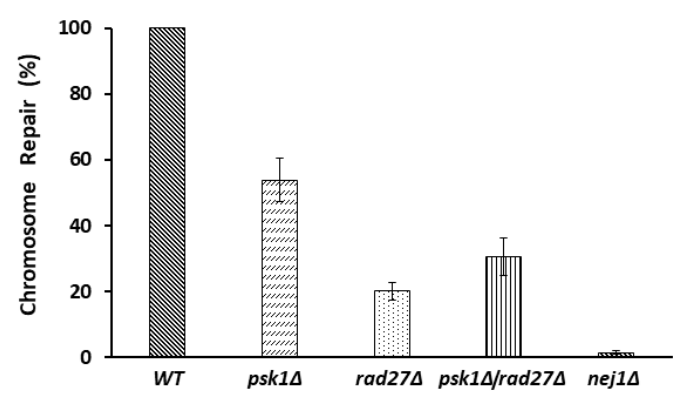

Figure 3.4: Examination of the functional relationship between RAD27 and PSK1 in DNA repair. (A-B) Spot test and growth curve analyses indicate that the overexpression of 
$R A D 27$ in a psk1 $\triangle$ background rescues sensitivity to MMS stress. (C-D) Double mutant analysis shows that the psk1 $\Delta / m r e 11 \Delta$ double mutant does not further impair plasmid or chromosomal repair efficiency beyond the rate of either single mutant. Genetic interactions were tested for using one-way ANOVA analysis (Dunnett's Multiple Comparison Test) between experimental samples $p<0.05$.

In Figure 3.4, a dosage suppression relationship is observed where RAD27 expression is capable of suppressing the damage-sensitive phenotype seen following the loss of the PSK1 gene while the double mutant does not show a significant deviation from the phenotype of either single deletion mutant.

\subsubsection{Repair fidelity}

The ability to repair breaks via NHEJ with relative accuracy is important considering the pathway is inherently error prone. To assess if the loss of PSK1 alters the rate of accurate repair, a plasmid-based fidelity assay was employed. In this assay, a dual selection plasmid is digested in the start codon of the KanMX6 cassette such that transferring of transformant colonies will grow on medium containing G418 if the break was accurately repaired. PSK1 mutants accurately repaired this plasmid $59 \%$ of the time compared to WT. This reduction in repair accuracy was greater than the rate seen in RAD27 mutants (81\%) but when both query genes were deleted, there was no significant additional reduction in fidelity (Figure 3.5). This supports previous findings in suggesting that PSK1 and RAD27 are affecting NHEJ through the same repair pathway. 
A

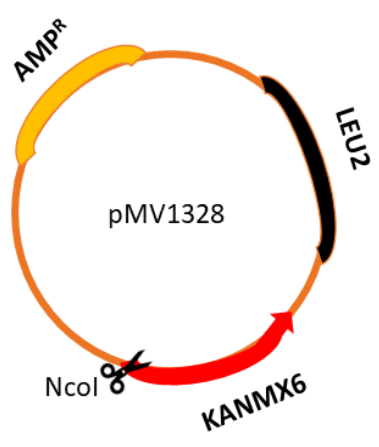

B

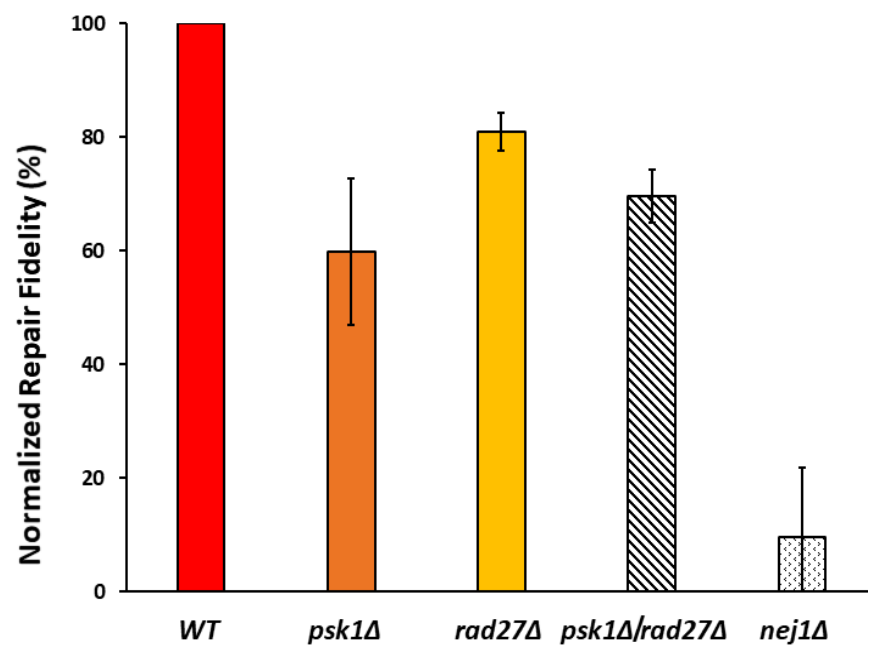

Figure 3.5: A dual selection plasmid-based fidelity assay (A) shows that $p s k 1 \Delta$ reduces repair fidelity but the $p s k 1 \Delta / \mathrm{rad} 27 \Delta$ double mutant does not show and additive reduction in repair accuracy. A Dunnett's multiple comparison test did not indicate a significant difference in the double mutant.

\subsection{Discussion}

The work herein has identified yeast PAS kinase as a new factor that influences NHEJ efficiency and fidelity. There has not previously been a role reported role for PSK1 in the NHEJ pathway but here we show that the loss of PSK1 function results in an impaired ability to resolve DSBs in both plasmid and chromosomal DNA (Figure 3.1). The use of global PPI prediction screening with PIPE suggested that Psk1 homolog Pim1 interacts with several proteins that influence DSB repair and cell cycle progression including CHK1, CHK2, HMGB1, ATK1 and BRCA2. This led to the inference that Psk1 may function in NHEJ ${ }^{66}$. After we found evidence to support a role for Psk1 in the NHEJ pathway, a series of genetic interaction analyses identified a possible functional 
association with $R A D 27$. We saw that the overexpression of $R A D 27$ rescues psk1 sensitivity to multiple DNA damaging agents. This novel relationship was further probed and showed that concurrent disruption of PSK1 and RAD27 do not produce additive phenotypes in the context of NHEJ repair (both chromosomal repair efficiency and repair fidelity) indicating that these two gene are likely functioning in the same genetic pathway.

Functional genetic analysis via SGA, like many other large-scale screening techniques, is subject to reasonably high rates of false positives and results are best interpreted by analyzing combined findings rather than individual genes ${ }^{215}$. Because of this, we compared our results to the DRYGIN database of previously reported yeast GIs and determined that $M R C 1$ had the most similar interaction profile ${ }^{216}$ suggesting that MRC1 and PSK1 may also function within the same pathway ${ }^{217}$. However, the overexpression of $M R C 1$ in a psk1 $\triangle$ background did not rescue the phenotype in all stress conditions (Table 3.2). Mrc1 is targeted by multiple kinases depending of cellular conditions. Psk1 phosphorylates Mrc1 while under oxidative stress, and Snf5 activates it under when under nutrient stress ${ }^{218}$. Mrc1 protects uncapped telomeres from excessive Exo1 exonuclease activity preventing an accumulation of ssDNA ${ }^{219}$.

Coupling these reported claims with the findings in this report, we propose a model wherein following DNA damage/oxidative stress, Psk1 is activated and phosphorylates Mrc1 which in turn limits exonuclease activity at break sites (Figure 3.6). This model is supported by our finding that $R A D 27$ rescues $P S K 1$ but the reverse is not 
true (Figure 3.4) and also that the overexpression of $M R C 1$ does not compensate for the loss of PSK1 (Table 3.2). Additionally, given the complex network responsible for Mrc1 activation, simply overproducing the protein would not necessarily result in the same downstream activity.

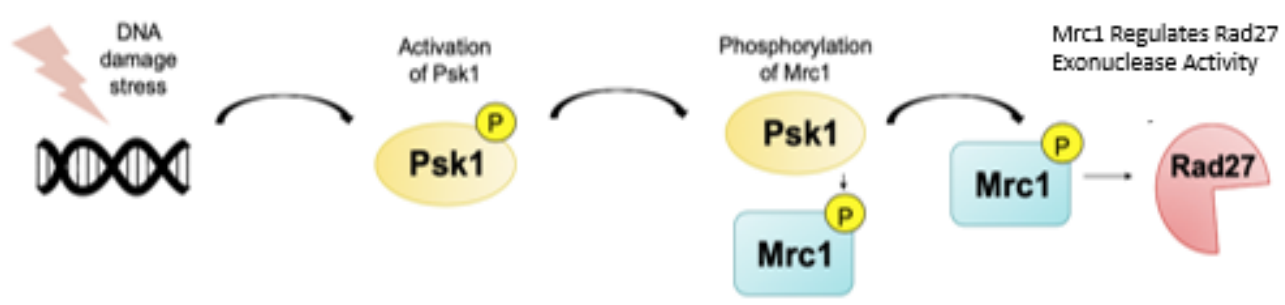

Figure 3.6. A potential mechanism for Psk1 and Rad27 in NHEJ where DNA damage stress activates Psk1 which in turn phosphorylates Mrc leading to regulation of Rad27 exonuclease activity.

An alternative explanation to the one provided in Figure 3.6 could be that in the absence of PSK1, ssDNA accumulates at uncapped telomeres. Functional Mrc1 protects uncapped telomeres from Exo-1 dependent nuclease activity ${ }^{219}$. Theoretically, if the loss of PSK1 results in SSDNA accumulation, it may titrate Yku and/or other NHEJ repair factors away from general NHEJ repair. This competitive inhibition model may also explain why RAD27 overexpression can compensate for the loss of PSK1 as multiple nucleases are proposed to function at DSB sites.

The inhibition of Psk1 by the synthetic peptides designed by InSiPS results in sensitivity to oxidative stress and UV light (Chapter 2 -Figure 2.3). Given the high sequence similarity to Pim1, this suggests that anti-Psk1 could potentially function as a 
Pim1 inhibitor as the proposed Psk1 binding site is directly adjacent to the Pim1 kinase domain.

\subsubsection{Summary}

Here we report evidence of a role for Psk1 in NHEJ repair. This role appears to be associated at least in part with the endonuclease activity of Rad27. Both RAD27 and PSK1 influence NHEJ repair fidelity but the loss of both genes does not further reduce repair efficiency or fidelity. Additionally, overexpression of RAD27 exonuclease rescues the reduces ability to respond to DNA damage stress seen in $p s k 1 \Delta$. We propose that the Psk1 PAS kinase is activated following certain types of DNA damage stress and functions through Mrc1 to regulate nuclease activity at break sites. However, the direct biochemical relationship between Psk1-Mrc1-Rad27 will require additional analysis. It would also be of interest to study the potential competitive inhibition model also discussed to determine if the availability of NHEJ repair factors at conventional DSB sites is affected by the accumulation at telomeres. In conclusion, Psk1 is an emerging participant in NHEJ and it is of interest to perform further studies aimed at understanding the specific role Psk1/Rad27 plays in the repair process. 


\section{Chapter 4: Actin-Related Protein 6 (ARP6) influences double-strand break repair induced chromatin remodeling in yeast}

\subsection{Abstract}

DNA double strand breaks (DSBs) threaten genetic integrity more than any other form of DNA damage. In eukaryotes, these structural lesions, produced by the severing of both phosphodiester backbones on a single DNA molecule, are either directly ligated through non-homologous end-joining (NHEJ) or resolved via homologous recombination (HR) using template DNA. For DSB repair to proceed, several histone-modifying and chromatin-remodeling proteins must activate to allow repair machinery access to the break site and facilitate communication with multiple signalling kinases. In S. cerevisiae, there are three primary complexes responsible for the initiation and regulation of chromatin remodeling following a DSB: i) INO80-C, which is primarily associated with HR, ii) SWR1-C, which promotes NHEJ, and iii) RSC-C, which is involved in both pathways as well as the general DNA damage response. Here we identify ARP6 as a factor involved in DSB repair through an RSC-C related pathway. The loss of $A R P 6$ significantly reduces NHEJ repair efficiency of linearized plasmids with cohesive ends, impairs repair of chromosomal breaks and sensitizes cells to DNA-damaging agents. Additionally, ARP6 mutants are also shown to improve the accuracy of NHEJ despite the associated reduction in repair efficiency. Genetic interaction analysis indicates that ARP6, MRE11 and RSC-C genes function within the same pathway and the overexpression of ARP6 
rescues $r s c 2 \Delta$ and $m r e 11 \Delta$ sensitivity to DNA damaging agents. Double mutants of $A R P 6$ and members of the INO80 and SWR1 complexes cause significant reduction in repair efficiency beyond expected rates, suggesting that $A R P 6$ functions independently of SWR1-C and INO80-C. These findings support a role for ARP6 in DSB repair that is independent of the SWR1 chromatin remodelling complex through an apparent RSC-C and MRE11 associated DNA repair pathway.

\subsection{Introduction}

DNA double-strand breaks (DSBs), structural shearing of a DNA molecule, can lead to genomic instability, chromosomal rearrangement, or cell lethality if ineffectively repaired ${ }^{220}$. Much research has focused on achieving a comprehensive understanding of DSB repair pathways because of their complex nature, biological importance and links to human diseases such as cancer ${ }^{221,222}$. In particular, several groups have used a yeast model to identify novel DSB repair genes ${ }^{124,131,132}$. Eukaryotic DSB repair proceeds primarily through one of two distinct pathways; homologous recombination (HR) which uses a homologous template and is considered error-free; or non-homologous endjoining (NHEJ), which directly re-joins the broken ends through an error-prone process.

S. cerevisiae has long served as an effective model for studying the molecular mechanisms underlying eukaryotic DSB repair because of highly conserved nature of the core repair machinery, relative ease of experimentation, and the ability to translate findings to the human system ${ }^{118,133,223}$. Three protein complexes comprise the core 
yeast NHEJ repair machinery required for initiation, end-bridging/processing, and ligation steps. The YKU complex (Yku70/Yku80) initiates NHEJ by forming a ring over each broken strand, which stabilizes the structure and protects the DNA from degradation. Next, the MRX complex (Mre11/Rad50/Xrs2) brings the DNA ends in close proximity and processes the strands in preparation for rejoining. Finally, the DNA Ligase IV complex (Dnl4/Lif1/Nej1) performs ligation by filling-in missing nucleotides and joining the broken ends ${ }^{224}$. Before any of these processes can occur, the DSB must be detected, repair factors must be recruited, and adjacent chromatin must be significantly modified to provide access to the break site while maintaining communication with the DNA damage response.

There is considerable cross-talk that occurs between DNA damage response proteins, cell cycle regulators, chromatin remodeling complexes and histone-related proteins throughout the repair process ${ }^{225}$. DSBs are first detected by sensors including the RSC (remodels structure of chromatin) complex and Mre11 ${ }^{93}$. Following detection, key DNA checkpoint proteins Mec1 and Tel1, orthologs of human ATR and ATM, are recruited to the break site and phosphorylate core histone protein $\mathrm{H} 2 \mathrm{~A}$ to form $\mathrm{\gamma}-\mathrm{H} 2 \mathrm{~A}$ 98. This phosphorylated $\mathrm{H} 2 \mathrm{~A}$ acts as a docking site for histone modifiers such as Arp4 and Esa1 and recruits various chromatin remodeling complexes involved in DSB repair. Three complexes are primarily responsible for chromatin remodeling following a DSB event: INO80-C, SWR1-C and RSC-C ${ }^{98-100 .}$ 
Following a DSB, INO80-C and SWR1-C are recruited to the site of damage in an Arp4-dependent manner ${ }^{99}$. Each complex promotes unique repair dynamics through its various subunits. INO80-C plays a predominant role in the repair of DSBs at replication forks and promotes end-resection to induce HR-dependent repair ${ }^{98}$. SWR1-C facilitates recruitment and binding of the YKU heterodimer to the break site in preparation for NHEJ repair ${ }^{102}$. Both INO80-C and SWR1-C facilitate the damage-induced exchange of $\mathrm{H} 2 \mathrm{~A}$ with its variant $\mathrm{H} 2 \mathrm{~A} . \mathrm{Z}$ which relaxes the chromatin structure to provide access for repair proteins 100,103 . In addition to these two key chromatin modifiers, the $\sim 17$ member RSC complex plays various roles in DSB repair including detecting breaks, signalling damage checkpoints, and facilitating nucleosome restructuring ${ }^{93,104}$.

As a chromatin remodeling complex, RSC-C mobilizes nucleosomes in response to DSBs and its activity is correlated with the level of H2A phosphorylation in a dosedependent manner ${ }^{226}$. RSC-C binds directly to nucleosomes and translocates along DNA ends, relaxing and releasing histones up and downstream of the break site ${ }^{226,227}$. Members of the RSC complex include Rsc1 and Rsc2 which interact with Yku80 and Mre11 and function in the process of NHEJ repair. The complex also contains two members of the highly conserved actin related protein (ARP) family, Arp7 and Arp9. Members of the ARP family are common to all three chromatin remodeling complexes. INO80-C contains Arp8, Arp5 and Arp4, SWR1-C contains Arp4 and Arp6, and RSC-C is reported to contain Arp7 and Arp9. 104,228,229. 
Arp6 is a lesser-studied member of the nuclear ARP family that has been reported to function both within, and independently of the SWR1 complex. It has been shown to regulate gene expression, often of ribosomal proteins, by exchanging of $\mathrm{H} 2 \mathrm{~A}$ for $\mathrm{H} 2 \mathrm{~A} . \mathrm{Z}^{230}$. Additionally, it has been indirectly implicated as a potential participant in NHEJ because of reported interactions with Swc2, Swc6 and Swc3, evidence that it may form a sub-complex within SWR1 as well as indications from large-scale analyses of DNA repair proteins; a direct role in DSB repair, however, has not been reported ${ }^{131,231}$. In this report, we investigated Arp6 as a potential participant in DSB repair. Once preliminary testing confirmed an important role for ARP6 in this pathway, we aimed to develop a general understanding of the role it may play in this process. Here we report a novel function for Arp6 in DSB repair through NHEJ that appears to be independent of its activities within the SWR1-C.

Our findings suggest that Arp6 plays an important role in promoting NHEJ by functioning with RSC-C and Mre11.

\subsection{Materials and Methods}

\subsubsection{Yeast strains and plasmids}

Mutant strains used are in a BY4741 background (MATa orf $\triangle:$ KanMX4 his3

leu2 $\Delta$ met15 $\Delta$ ura3 $\Delta)^{205}$. ARP6 was deleted in a Y7092 background (MATa

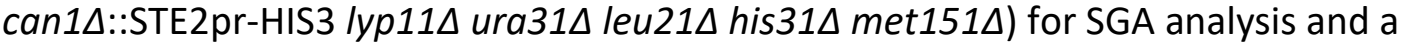
JKM139 background (MATa $h m r \Delta:: A D E 1 ~ h m l \Delta:: A D E 1$ ade1-100 leu2-3,112 lys5 
trp1::hisG ura3-52 ade3::GAL-HO) for chromosomal break and repair fidelity analysis, through a PCR-based transformation in which ARP6 was replaced by Nat ${ }^{R 209}$. Plasmid repair assays were carried out using $\mathrm{p} 416$ containing a URA3 marker using Xbal digestion. A blunt end repair assay was carried out using YCplac111 with LEU2 marker using Smal digestion. Plasmid pMV1328 carrying a LEU2 marker and Amp ${ }^{\mathrm{R}}$ was used for the repair fidelity assay ${ }^{124}$. Derivatives of pGEH expression plasmids were used in suppression analysis via galactose induction ${ }^{206}$. Plasmid pGV-255/LIVE and its derivative pGV-256/DEAD were used for the homologous recombination assay ${ }^{232}$

\subsubsection{Plasmid repair assay}

Ycplac111 and p416 were single-digested at Smal and Xbal sites, respectively. These sites have flanking regions that lack homology to yeast chromosomal DNA and require NHEJ repair for proliferation. Parallel transformation of digested and intact plasmid was carried out in WT and mutant strains. After three days of growth on synthetic minimal medium lacking uracil or leucine, colonies were counted. The ratio of colonies formed by digested transformants to circular transformants in mutant strains, normalized to WT, was used to assess the repair efficiency 207,208 .

\subsubsection{Repair fidelity assay}

Plasmid pMV1328 was digested by Pstl within its KanMX6 marker. Digested and circular plasmids were transformed into mutant and WT strains in JKM139 background. Cells were grown on synthetic medium lacking leucine for 2-3 days. To test the accuracy 
of repair, 50-100 transformants (colonies) were grown on YPD medium containing G418 $(200 \mu \mathrm{g} / \mathrm{mL})$. Growth on $\mathrm{G} 418$ represented accurate repair ${ }^{132}$. Repair fidelity is represented as the ratio of viable cells over total transformants transferred and normalized to the WT ratio.

\subsubsection{DNA damaging drug-sensitivity analysis}

Mutant and WT strains from a BY4741 background were grown to saturation in $5 \mathrm{~mL}$ of YPD medium for 2 days. Saturated cultures were serially diluted $10^{-1}-10^{-4}$ and $15 \mu \mathrm{l}$ of each dilution was spotted on YPD medium containing $3 \mu \mathrm{g} / \mathrm{ml}$ phleomycin (PHLM) or $70 \mathrm{mM}$ hydroxyurea (HU). Viability of each strain was visually examined after 2-3 days of growth at $30^{\circ} \mathrm{C}$.

\subsubsection{Genetic interaction analysis}

Synthetic genetic array (SGA) analysis was performed as in Tong et al, 2006209 and phenotypic suppression analysis (PSA) was performed as in Sopko et al, $2006{ }^{206}$. Strains carrying deletion or overexpression of ARP6 in Y7092 background were crossed to a DNA Damage containing deletion strains of 384 genes associated with DNA repair, DNA damage response, cell cycle, and chromatin remodeling. The resultant strains were then grown on medium containing $30 \mathrm{mM} \mathrm{HU}$ and $1.6 \mu \mathrm{g} / \mathrm{mL}$ PHLM to test for conditional genetic interactions. Results were visually scored. Each experiment was repeated three times and the average results are presented. 


\subsubsection{Chromosomal repair assay}

A JKM139 background was used to examine efficiency of repair of chromosomally induced breaks. In this strain, a HO site-specific endonuclease under a GAL promoter induces DSBs at the MAT locus. HML and HMR, homologous regions of MAT are deleted to prevent the possibility of HR repair, which allows the evaluation of NHEJ repair efficiency of chromosomal breaks ${ }^{208}$. WT, mutants and mutants carrying overexpression plasmids were grown in YPD or selective medium to saturation and were then serially diluted to concentrations of $10^{-1}$ to $10^{-4} .15 \mu$ l of each dilution was spotted on YP-Galactose and YPD. Fitness was visually assessed after 3 days of incubation at $30^{\circ} \mathrm{C}$.

\subsubsection{Homologous recombination assay}

In this assay, a pGV-256/DEAD plasmid was digested at its non-functional lacZ gene with BgIII ${ }^{232}$. Separately, a PCR-product containing functional lacZ was amplified using the pGV-256/LIVE plasmid to provide a potential template for HR repair. 10ng of 'dead' plasmid and 200ng of PCR product containing the functional lacZ cassette were co-transformed into mutant and WT strains and grown for 2 to 3 days on selective media. A minimum of 50 colonies were transferred to a new plate and grown for 1 day. To measure the frequency of HR repair, a colony-lift assay was performed by transferring colonies onto a nitrocellulose membrane and lysing with liquid nitrogen, prior to incubation in Z-buffer containing X-gal (5-bromo-4-chloro-3-indolyl- $\beta$ - $d$ - 
galactopyranoside) at $30^{\circ} \mathrm{C}$ for $\sim 4$ hours ${ }^{208}$. The frequency of blue colonies was normalized to the WT ratio to assess HR event frequency.

\subsection{Results}

\subsubsection{Assessment of ARP6 function in DNA repair}

Because Arp6 has been loosely linked to NHEJ, we first investigated if ARP6 influences the NHEJ pathway using a series of DNA repair assays. A classic assay for investigating the role of a gene in NHEJ repair is to investigate the effect of a gene deletion on the efficiency of rejoining linearized plasmids ${ }^{233}$. A deficiency in the ability to ligate a digested plasmid that lacks homology to intracellular DNA by a gene mutant is an indication of involvement of the deleted gene in NHEJ repair. This plasmid repair assay can also assess the repair of multiple break structures, including blunt and cohesive ends, depending on the restriction enzyme used ${ }^{124}$. When ARP6 mutants were presented with breaks possessing cohesive overhangs (Xbal digestion), repair efficiency was reduced to $41 \%$ compared to WT (Figure 4.1.A), supporting previous findings and indicating that ARP6 may contribute to efficient NHEJ repair.

We also examined the ability of arp6 6 to repair blunt-end breaks. The deletion of ARP6 enhanced repair of blunt-end breaks compared to WT resulting in an efficiency of $180 \%$ (Figure $4.1 . B$ ). In yeast, blunt-end breaks are repaired through an alternative YKUindependent mechanism ${ }^{124,132}$. This observation suggests a role for ARP6 in classic NHEJ repair of breaks with cohesive ends, and not those with blunt ends. The fact that 
deletion of ARP 6 increased the efficiency of blunt end repair merits further investigation of a potential suppressing function for ARP6 that may regulate the repair of breaks with no overhangs. If $A R P 6$ functions to promote NHEJ this activity is likely independent of YKU binding. It is also noteworthy that deletion of YKU70 enhances blunt-end repair efficiency, a trend that is not seen with other key NHEJ genes $124,133,234$.

Plasmid DNA and chromosomal DNA molecules differ in their topology which may lead to variances in repair dynamics ${ }^{235}$. To assess if $A R P 6$ also influences the repair of severed chromosomes, a chromosomal break assay was performed. This assay utilizes a JKM139 background which possesses a single-locus specific HO-endonuclease regulated by a GAL promoter, which continuously induces DSBs at the MAT locus when in the presence of galactose ${ }^{236}$. These breaks must be repaired via NHEJ as the target sequence is not homologous to other chromosomal regions. A severe reduction in the survivability of arp6 $\Delta$ cells, was observed following consistent chromosomal DSB induction (Figure 4.1.C) suggesting that ARP6 is important in repair of chromosomal breaks and further supporting its involvement in NHEJ.

To further analyze if $A R P 6$ is an important participant in DNA repair activities, we subjected it to drug sensitivity analysis using DNA-damaging agents. The sensitivity of gene mutants can be used as an indicator for involvement in DNA repair ${ }^{177}$. The loss of important DNA repair genes generally, but not necessarily, increases the vulnerability of cells to DNA damaging drugs ${ }^{234,237}$. Most drug sensitivity assays involve the induction of persistent DNA damage stress which invokes a comprehensive damage response 
involving a range of repair pathways. The damage induced by the agents employed in this study do not exclusively result in DSBs but rather induce stress events that eventually lead to DSBs, in addition to other forms of DNA lesions. HU depletes the dNTP pool, which eventually results in the collapse of replication machinery and the induction of double strand breaks at replication forks ${ }^{83}$. PHLM is believed to intercalate DNA directly and promote the production of free radicals that react to form a variety of DNA lesions, including DSBs ${ }^{82}$. Consistent with an involvement for ARP6 in DNA repair, cells with deletions of ARP6 were sensitive to PHLM ( $3 \mu \mathrm{g} / \mathrm{ml})$ and HU (70 mM) (Figure 1D).
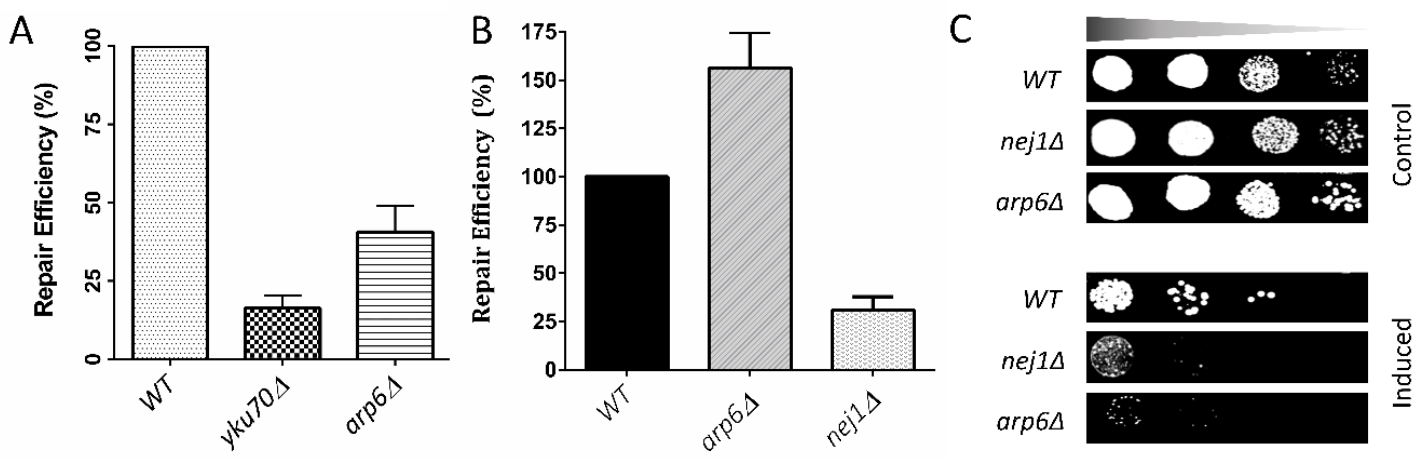

D

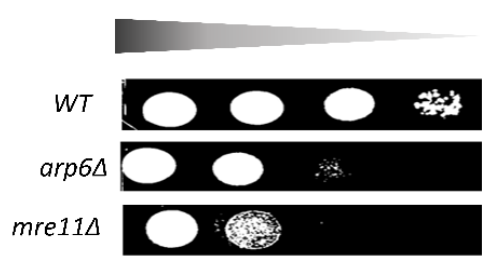

70mM Hydroxyurea

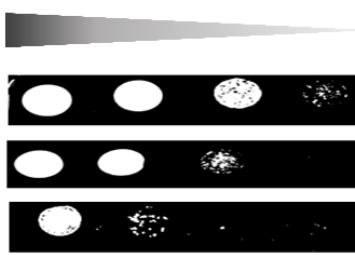

$3 \mu \mathrm{g} / \mathrm{mL}$ Phleomycin

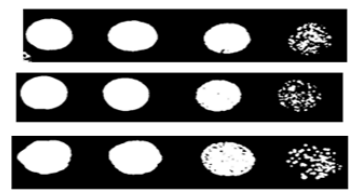

Control

Figure 4.1: Plasmid repair, chromosomal break, and drug sensitivity assays support a role for ARP6 in NHEJ repair. (A) Efficiency of repair of a linearized (Xbal digestion) p416 plasmid with cohesive ends via NHEJ is reduced to $40 \%$ when ARP6 is deleted. $y k u 70 \triangle$ is used as a control (B) Contrarily, when YcPlaclll is digested to produce blunt ends (Smal digestion), deletion of ARP6 increases repair efficiency (>60\% more efficient than WT). 
Plasmid repair assays were repeated 5 times and error bars indicate standard deviation amongst trials. (C) Chromosmal break assay indicates that deletion of ARP6 sensitizes cells to repeated chromosomal breaks. Error bars indicated standard deviation. (D) Drug sensitivity analysis. Deletion mutants for ARP6 are sensitive to DNA damaging agents phleomycin $(3 \mu \mathrm{g} / \mathrm{ml})$ and hydroxyurea $(70 \mathrm{mM})$. Cells were grown to saturation and serially diluted before spotting on medium with and without drug. NEJ1 mutants are used for (B) as the YKU complex is not believed to be involved in the repair of blunt-end breaks.

\subsubsection{ARP6 effects repair fidelity}

The loss of ARP6 increases NHEJ accuracy. NHEJ is considered to be an errorprone pathway that can introduce mutations within the repaired region of the DNA ${ }^{238}$. However, the genetic composition of repair factors can significantly influence the errorrate of NHEJ repair ${ }^{239}$. We examined the role of $A R P 6$ in facilitating accurate NHEJ repair by subjecting $\operatorname{arp} 6 \Delta$ to a repair fidelity assay.

In this dual-selection assay, a plasmid digested within its KanMX6 cassette is used to indicate the rate of accurate NHEJ repair based on the ability to grow on G418.

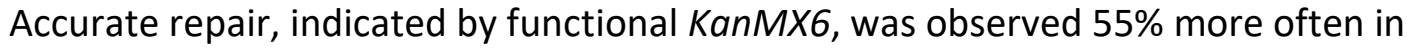
arp6 $\Delta$ mutants compared to WT (Figure 4.2). The efficiency of repair of the same plasmid was reduced by $82 \%$ in arp $6 \Delta$ cells, which is consistent with our previous finding that ARP6 impairs repair efficiency of cohesive ends. This suggests that even though deletion of ARP6 causes defects in the efficiency of the repair process, NHEJ proceeds through an alternative mechanism with very high accuracy. Furthermore, these results indicate that ARP6 may affect the balance between efficiency and accuracy of repair, suggesting its function may be in a regulatory capacity independent of the core repair machinery. Several proteins can influence NHEJ fidelity but most important factor in 
fidelity appears to be the MRX complex. MRX influences multiple pathways that promote the timely dissociation of YKU, an event shown to lead to accurate repair ${ }^{97}$.

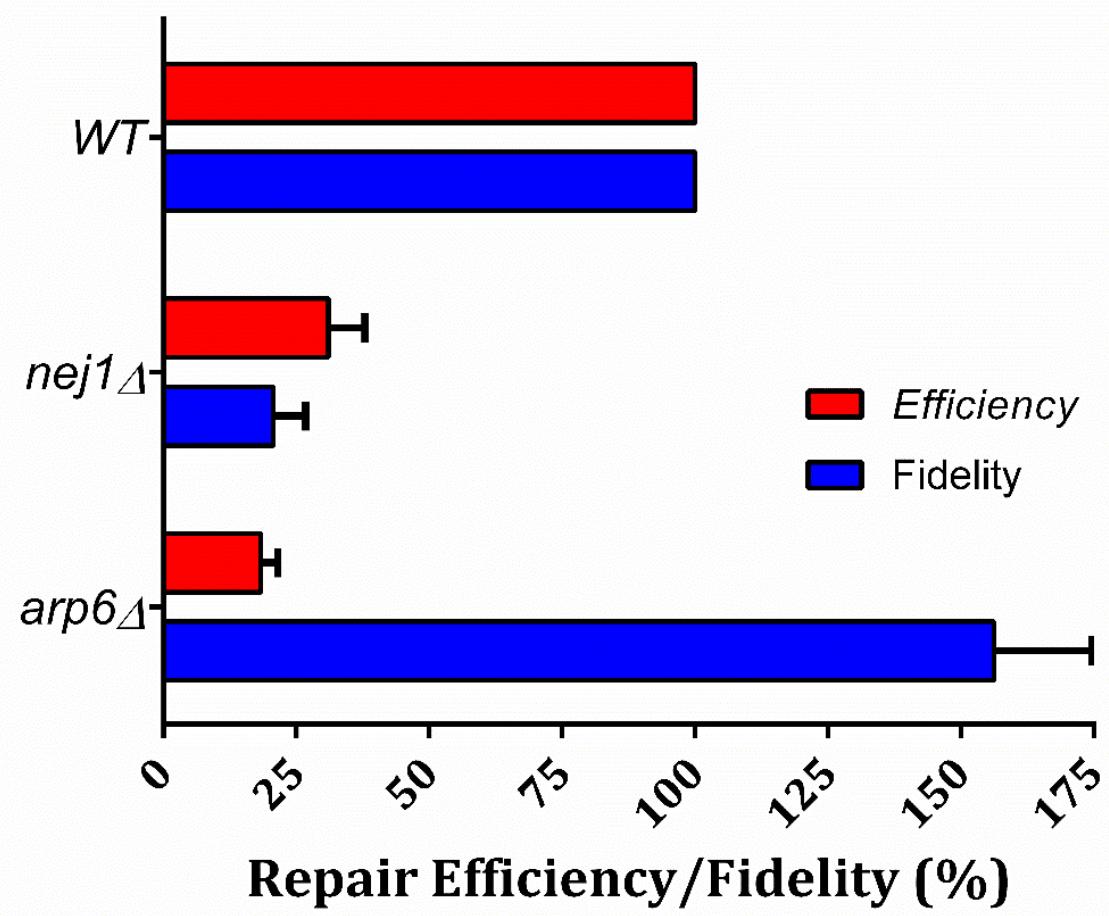

Figure 4.2: Loss of ARP6 results in less efficient but more accurate repair. arp6 $\Delta$ decreases the efficiency of NHEJ repair of linearized plasmid pMV1328 (18\%) and drastically increases the accuracy of repair by $55 \%$. A dual-selection plasmid repair assay was repeated five times and error bars indicate standard deviation amongst trials.

The observation of increased repair fidelity may help explain the severe sensitivity of arp6 $\Delta$ found in our chromosomal break analysis, where cells were unable to recover from the repeated induction of chromosomal DSBs (Figure 4.1.C). Fidelity of repair plays an important role in this assay, as mutations in the recognition site cause the cells to become resistant to further assault by HO-endonucleases. Consequently, the 
extreme phenotype observed for ARP6 deletion mutants could be explained by a decrease in repair efficiency combined with an increase in repair fidelity, which would leave break sites prone to increased rounds of continual assault.

\subsubsection{Interaction analysis suggests a role with DNA repair and chromatin remodeling}

To better understand how ARP6 functions within DNA repair, we examined how it interacts with other known DNA repair genes using a variety of genetic interaction (GI) and conditional GI analyses. GI network analysis is used in functional genomics to investigate novel gene functions and/or to elucidate the role of genes in various cellular pathways ${ }^{43}$. Furthermore, conditional GI analysis can also be employed to identify interactions that only occur under controlled stress conditions, such as interactions that only occur under DNA damage stress ${ }^{33}$. Specifically, negative interactions resulting from the deletion of two genes can be identified as a synthetic sick or lethal phenotype and may suggest that the target genes function in compensating, and often parallel pathways, that perform similar functions ${ }^{43,206}$.

We performed synthetic genetic array (SGA) analysis and phenotypic suppression analysis (PSA) on ARP6 using a DNA Damage Array containing 384 genes implicated in DNA repair, DNA damage response and other related pathways. SGA analysis involved the systematic crossing of an $\operatorname{arp} 6 \Delta$ single deletion strain with an array of single mutants, to produce double mutants ${ }^{209}$. Our analysis showed that ARP6 has negative genetic interactions with several NHEJ genes, namely RAD50, RTT109 and 
RAD27 (Figure 4.4.A). In addition to these NHEJ genes, ARP6 also interacts with key genes involved in the DNA damage response $A R P 8, R A D 53$ and $D D C 1$, mismatch repair MLH1 and $M L H 2$, and post-replication repair RAD18. Interestingly, under DNA damaging conditions, negative genetic interactions were observed between ARP6 and key HR genes RAD55 and RAD52 suggesting a DNA damage induced functional relationship with HR genes.

A

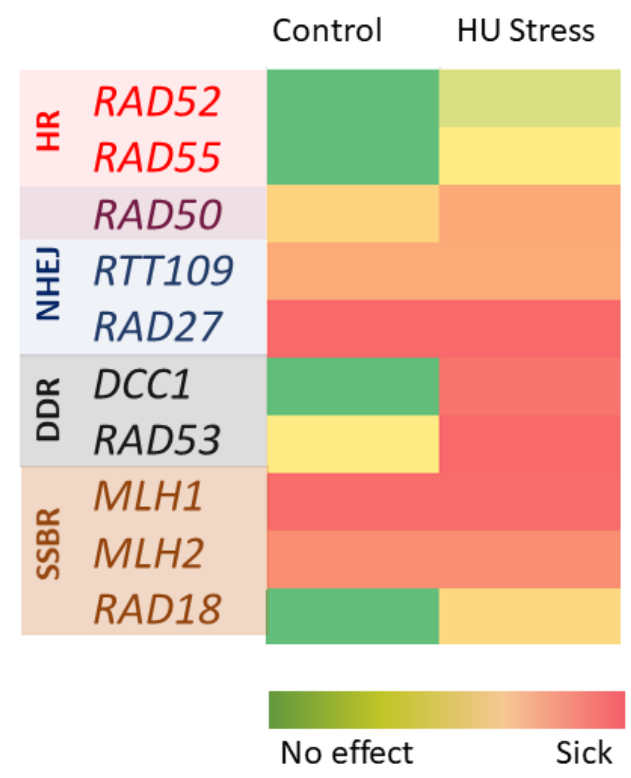

Fitness
B

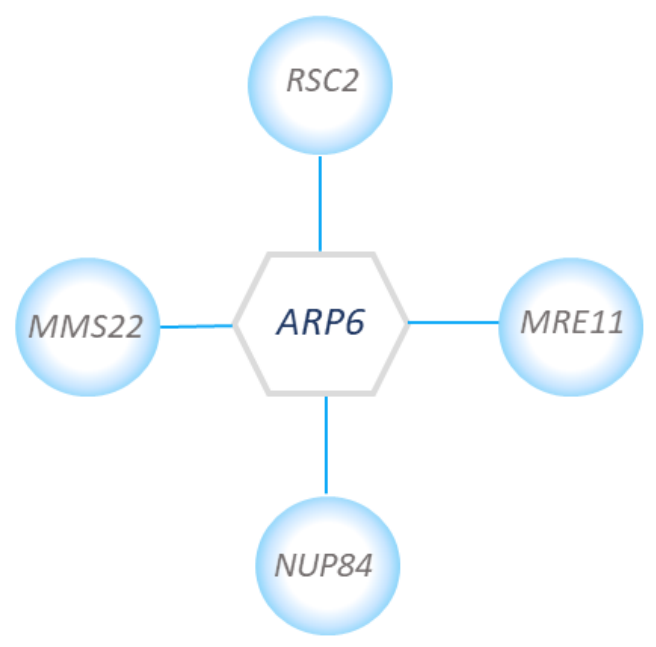

Figure 4.3: Genetic interaction profile for ARP6. (A) Genetic interactions of ARP6 include genes from various DNA repair pathways. SGA analysis of double mutants of ARP6 and various genes involved in the DNA damage response, DNA repair pathways, and chromatin remodelling were analyzed under drug-free conditions and under $30 \mathrm{mM}$ hydroxyurea. (B) Overexpression of ARP6 compensates (rescues) sensitivity of MMS22, MRE11, RSC-C2, and NUP84 mutants. 
The GI network of ARP6 which included genes involved in HR, NHEJ, and the DNA damage response, hints to a potential role for ARP6 upstream of NHEJ repair machinery. SGA analysis suggested a role for ARP6 in various DNA repair pathways, a result that is not unexpected if Arp6 is involved in repair-related chromatin remodeling of DNA damage signalling. Additionally, analysis of known Arp6 protein-protein interactions indicated a significant enrichment of proteins associated with both SWR1-C and INO80 (Figure 4.5). To further hone-in on the specific function of ARP6, PSA was performed. PSA involves overexpressing one gene in a gene deletion background under stress conditions to examine if the overexpressed gene can "rescue" the sensitive phenotype of the deletion mutant ${ }^{240}$. Phenotypic rescue is often observed in genes which perform similar functions within a pathway ${ }^{210}$. For example the overexpression of $P P H 3$ or PSY2 was previously shown to compensate the sensitivity of CHK1 deletion to DNA damaging conditions ${ }^{207}$. To this end, we introduced conditionally-expressed ARP6 overexpression plasmids into the 384 single gene deletion mutants in the DNA Damage Array. Under DNA damage induction using PHLM and HU, ARP6 overexpression compensated for sick phenotypes of four interesting gene deletion strains which helped suggest a possible function in NHEJ.

\subsubsection{Overexpression of $A R P 6$ rescued four deletion mutants that were sensitive to DNA damaging drugs}

The overexpression of $A R P 6$ compensated for MMS22, a stabilizer of replication forks, MRE11, a key NHEJ and DNA damage response gene, RSC2, a key component of 
the RSC-C chromatin remodeling complex, and NUP84, a member of the nuclear pore complex involved in translocating chromosomes to the nuclear periphery for DSB repair. Both NUP84 and MMS22 are associated with key DSB repair-related chromatin remodelling proteins. For example, NUP84 is known to genetically interact with both $A R P 6$ and $A R P 8^{46}$ while MMS22 has known physical interactions with three members of the RSC complex, Rsc8, Rsc9 and Arp9 ${ }^{241}$. These results further support the implication of $A R P 6$ in the repair pathway upstream of DSB repair and suggest a possible connection between ARP6, MRE11 and the RSC-C chromatin complex.

\subsubsection{ARP6 function appears related to the RSC-C chromatin remodeling}

\section{complex}

Following a DSB, regulation of repair is largely controlled by H2A.Z (coded by HTZ1), a variant of H2A which is incorporated at the break site ${ }^{102}$. Recruitment of various chromatin remodelling complexes following these events can direct repair dynamics. SWR1-C promotes NHEJ and is required for response to DNA damage and efficient binding of YKU. Additionally, INO80-C, which promotes HR, and RSC-C, which is involved in both HR and NHEJ, are also involved in chromatin remodelling following a DSB. To study the role of ARP6 in NHEJ, we examined its functional relationship with members of SWR1-C, INO80-C and RSC-C chromatin remodeling complexes, in addition to Htz1. This was done by analyzing double deletion mutants of ARP6 and key members of each of the three relevant complexes. 
Since Arp6 has been reported to be a part of SWR1-C, we first investigated the functional relation between the ARP6 gene and genes encoding members of SWR1-C, SWC2 and SWR1, in re-joining of linearized plasmids (Figure 4.4.B). Double deletion mutants $\operatorname{arp} 6 \Delta /$ swr1 $\Delta$ and $\operatorname{arp} 6 \Delta /$ swc2 $\Delta$ were subjected to the plasmid repair assay and compared to single mutants of the relevant strains to determine if genetic interactions were occurring. The premise of this experiment is that if two genes participate in the same pathway, their double deletion should not produce a phenotype significantly different from the two single gene deletions. However, if the genes affect the same process via different pathways, a combinatory effect should be observed, where the double deletion causes a more severe effect than the individual gene deletions.

Deletion of SWC2 reduced the repair efficiency to 50\%, roughly comparable to the $41 \%$ efficiency observed in the arp $6 \Delta$ background. However, the double deletion of ARP6 and SWC2 (arp6 $4 / s W c 2 \Delta)$ significantly impairs the repair process and produced a synergistic effect that resulted in only $8 \%$ repair efficiency compared to $W T$, suggesting their involvement in additive pathways (Figure 4.B). In the case of SWR1 single deletion, repair efficiency was calculated at 56\%, while double deletion of ARP6 and SWR1 $(\operatorname{arp} 6 \Delta / \mathrm{swr} 1 \Delta)$ further reduced the repair efficiency to $20 \%$. These data showed the additive influence of $A R P 6$ with SWR1 and SWC2, suggesting that during NHEJ repair, ARP6 likely functions in a parallel pathway to SWR1-C. This functional relationship suggests that $A R P 6^{\prime} s$ cumulative influence on NHEJ appears independent of the SWR1 complex. 
A

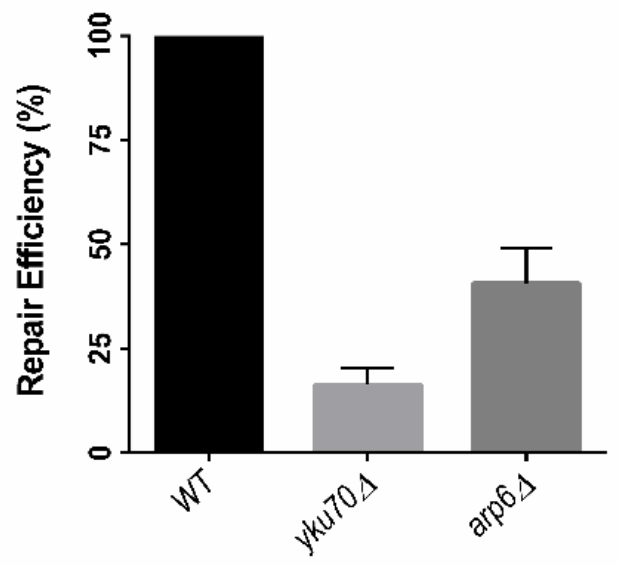

C

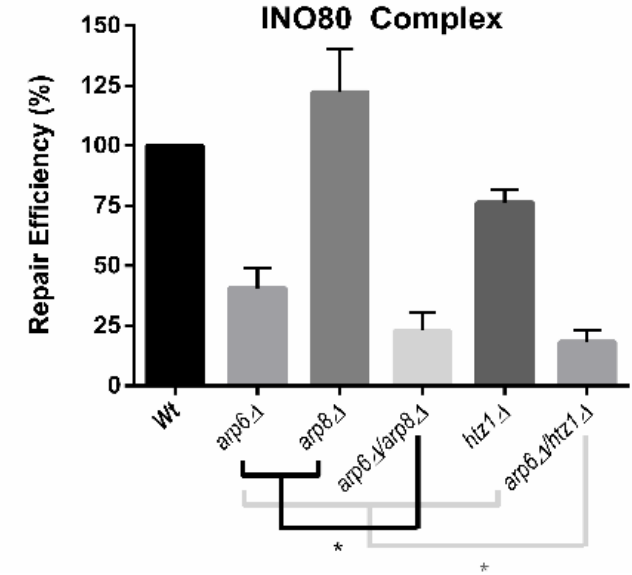

B

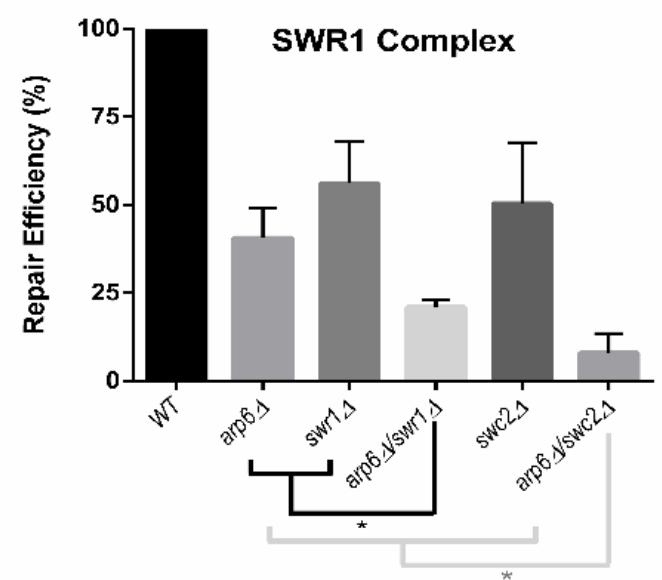

D

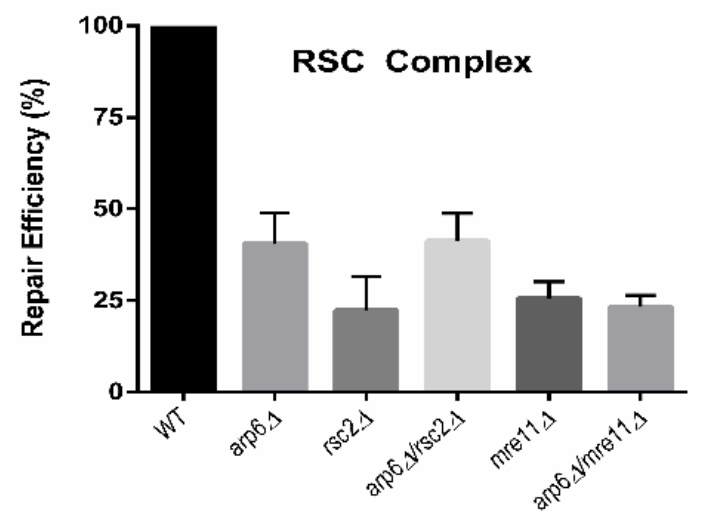

Figure 4.4: Genetic interaction analysis suggests $A R P 6$ is functionally related to RSC-2 and MRE11 in the context of NHEJ repair. (A-D) Plasmid repair analysis of linearized p416 with overhangs. Single and double mutant repair efficiencies is used to analyzes functional genetic relationships. Comparison of repair efficiencies of ARP6 single mutants to the repair efficiencies of double mutants of genes involved in the SWR1 (B), INO80 (C) and RSC (D) complexes was performed using one-way ANOVA analysis (Dunnett's Multiple Comparison Test) post hoc. There is no significant reduction ( $p>0.05$ ) in double mutants of genes in the RSC-C complex (MRE11 and RSC2) indicating that ARP6 function in NHEJ is related to the role of RSC-C (D).

Next, we examined whether the role that ARP6 plays in NHEJ is related to functioning of the INO80 complex or generalized chromatin remodelling following DNA damage via HTZ1 (Figure 4.4.C). ARP8 is an important component of INO80-C and arp8D 
mutants have been used to represent INO80 disruption for phenotypic analysis ${ }^{98}$. We investigated the repair efficiency of double mutants of $A R P 6$ with ARP8 and HTZ1. Our results show that although deletion of ARP8 did not reduce the efficiency of NHEJ repair compared to WT, the double deletion of $A R P 8$ and $A R P 6$ lowered the repair efficiency to 23\%, whereas the ARP6 single deletion displayed 41\% efficiency (Figure 4.4.C).

Furthermore, HTZ1 single mutants show moderate reduction in repair (76\%) while the double deletion of ARP6 and HTZ1 significantly lowered the repair efficiency to $18 \%$. Together these observations suggest that $A R P 6^{\prime}$ s role in NHEJ is independent of the INO80-C and HTZ1 pathways.

We then investigated the role of ARP6 in association with RSC-C. In addition to its traditional role in transcription regulation, RSC-C functions as an early sensor of DSBs, an activator of the DNA damage response, and an ATP-dependent chromatin remodeler in both NHEJ and HR ${ }^{93}$. RSC-C responds rapidly to DSBs and recruits key DNA damage response proteins Tel1, Mec1 and Rad9. It also interacts physically with Mre11, the earliest sensor of DSBs. RSC-C's function in DSB appears to primarily be involved with the earliest stages of repair.

Normalized repair efficiency of $r s c 2 \Delta$, a key component of the RSC-C complex was $22 \%$, which is not significantly different than the repair efficiencies of arp6 $\Delta$ and $\operatorname{arp} 6 \Delta / r s c 2 \Delta$ which are both $41 \%$ (Figure 4.4.D). This type of non-additive phenotype is often indicative of genes which encode members of the same non-essential pathways ${ }^{43}$. The genetic relationship between ARP6 and RSC2/MRE11 suggests that the role of ARP6 
in NHEJ may be related to RSC-C activities. Furthermore, mre $11 \Delta$ and $\operatorname{arp} 6 \Delta / m r e 11 \Delta$ showed comparable repair efficiencies at $26 \%$ and $23 \%$ respectively (Figure $4.4 . \mathrm{D}$ ) suggesting that ARP6 and MRE11 function within the same pathway ${ }^{43}$. The deletion of ARP6 did not have an additive effect on the reduced efficiency of NHEJ caused by the deletion of RSC2 or MRE11. Together, these results suggest possible functions at the early stages of damage sensing, repair factor recruitment or early chromatin remodelling.

To further study the functional association between ARP6 and the RSC-C pathway, we investigated the drug sensitivity profiles of single and double mutants. Double mutants $\operatorname{arp} 6 \Delta / r s c 2 \Delta$ and $\operatorname{arp} 6 \Delta / m r e 11 \Delta$ rescue this sensitivity and increase fitness through an apparent phenotypic suppression that results in cells that phenocopy the WT. In this case, the deletion of a second gene (RSC2 or MRE11) relieve the cell requirement for the presence of another compensatory gene resulting in a positive genetic interaction (suppression) ${ }^{242}$. This asymmetric positive interaction provides indirect evidence that $A R P 6$ and $M R E 11 / R S C-2$ may perform related functions in response to severe DNA damage that are not limited to NHEJ ${ }^{242}$.

\subsubsection{Loss of ARP6 severely reduces the efficiency of homologous}

\section{recombination}

RSC-C and MRE11 participate in the earliest stages of DSB repair including break detection and damage signalling that can induce either NHEJ or HR repair. Because RSC- 
C and MRE11 participate in both pathways, it is important to understand how ARP6

effects HR efficiency. To asses this, ARP6 mutants were subjected to a homologous recombination assay. This assay is a variation of the previously described plasmid repair assay, but the vector used is digested within a non-functional lacZ gene. Linearized plasmids are co-transformed with excess amount of a PCR-amplified linear cassette encoding a functional lacZ ${ }^{232}$. Cells that have integrated the cassette via HR will produce a blue colour in the presence of X-gal solution. Repair through NHEJ is indicated by a colony remaining white in colour. ${ }^{208}$. The relative frequency of HR or NHEJ is reflected by the ratio of blue and white colonies/total colonies, normalized to the WT frequency.

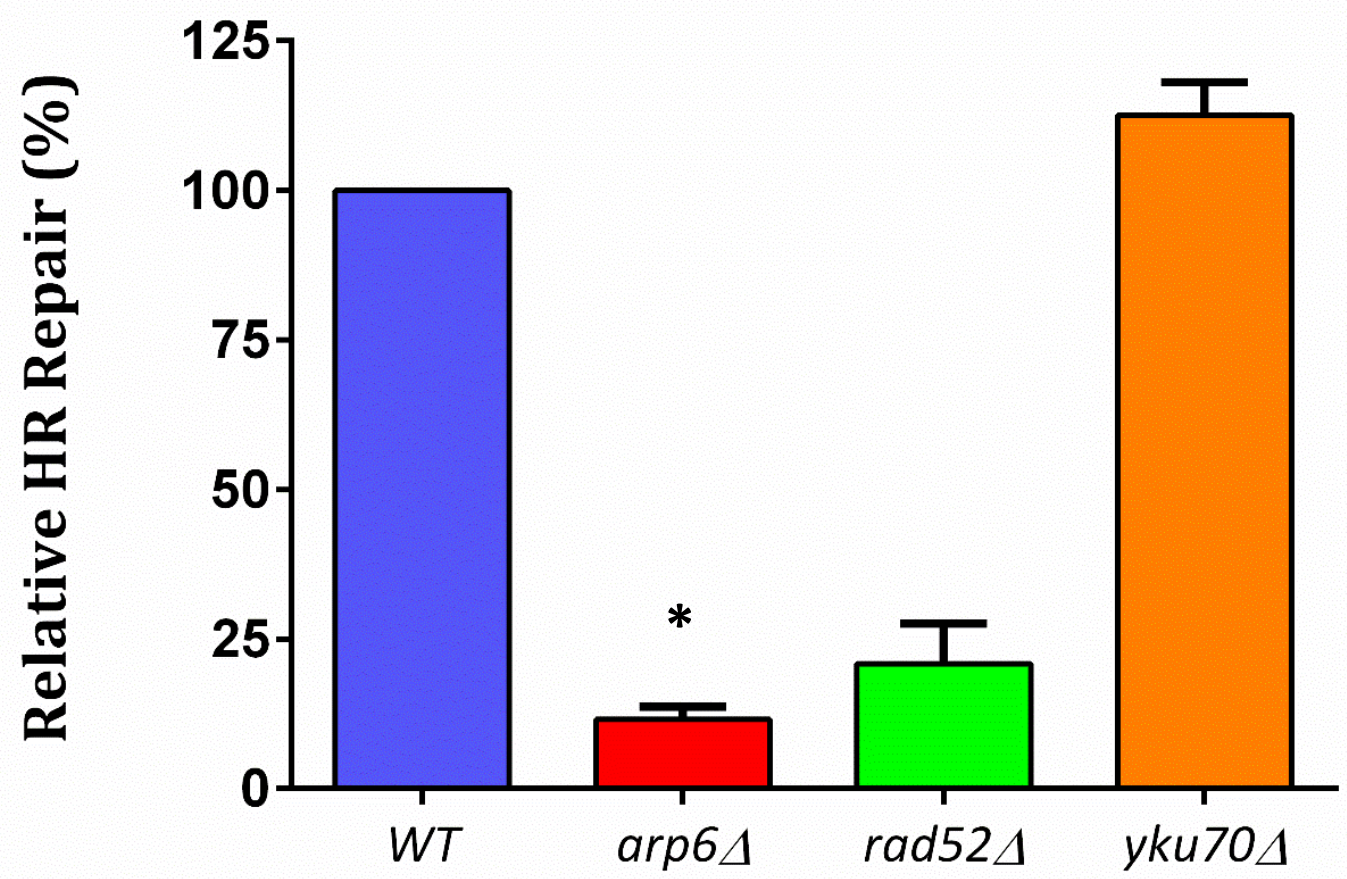

Figure 4.5: The deletion of ARP6 pushes DSB repair towards the NHEJ pathway. A plasmid-based assay evaluates repair pathway choice for single mutants compared to 
WT. HR efficiency is presented as the ratio of blue/total colonies normalized to the blue/total ratio of the WT +/- SD. Significant change in relative HR repair efficiency determined by T-test, $p<0.05$.

Deletion of ARP6 severely depressed the recombination repair rate, with only $12 \%$ of repair events occurring via HR compared to the WT ratio (Figure 4.5). Efficiency of $H R$ in arp6 6 is even lower than positive control rad52 in which HR accounts for $24 \%$ of repair events. If $A R P 6$ function in repair is strictly related to its role within SWR1-C, one might expect that the loss of ARP6 would lead to increased rates of HR. SWR1-C promotes the binding of YKU thereby facilitating NHEJ, so if arp6 4 causes no other effects other than decreased SWR1-C functionality, NHEJ should be impaired ${ }^{98}$. However, the results in Figure 4.5 indicate that $\operatorname{arp} 6 \Delta$ mutants promote NHEJ and/or are severely deficient in HR repair which again supports our claim that $A R P 6$ functions in DSB repair independently of SWR1-C or in addition to its role within that complex.

\subsection{Discussion}

The evidence presented herein indicates a novel function for ARP6 in DSB repair through the RSC-C complex and MRE11. Members of the ARP family have general sequence homology but vastly different functions within the cell. In yeast, the ARP family is comprised of 10 members including cytoplasmic proteins Arp1, Arp2, Arp3, and Arp10 and nuclear proteins Arp4, Arp5, Arp6, Arp7, Arp8, and Arp9 ${ }^{243}$. Some ARP proteins such as Arp8 and Arp5 were previously shown to be involved in the DNA repair 
process through their role in the INO80 complex ${ }^{101}$. Arp4 is an essential protein required for chromatin remodeling at DSB sites ${ }^{244}$. Arp6 is a lesser-studied member of the ARP family and a specific role for Arp6 in DNA repair has not been reported previously. However, certain evidence has indirectly implicated it as a potential participant in DSB repair such as sensitivity to methyl methanesulfonate (MMS) and a reported increase in recombination frequency when ARP6 is deleted ${ }^{243}$.

Kawishma et al, 2007 reported that the deletion of ARP6 inhibits the function of the SWR1 complex and has no effect on HR efficiency but causes minor increases in the frequency of spontaneous and MMS-induced unequal sister chromatid recombination events. This increase in recombination frequency was attributed to a dysregulation of $\mathrm{H} 2 \mathrm{~A} \leftrightarrow \mathrm{H} 2 \mathrm{~A} . \mathrm{Z}$ exchange which was proposed to lead to an increased frequency of recombination events. However, an alternative explanation could be that the loss of ARP6 promotes NHEJ which may be mutagenic and lead to an increased rate of secondary HR events and our study provides some preliminary evidence to support this hypothesis.

Here we report a potential role for ARP6 in NHEJ linked to RSC-C activities through its genetic association with $R S C 2$. This result is not surprising since ARP6 has been reported to function independently of SWR1-C in other processes such as transcription regulation ${ }^{230}$. Arp6 may function alone, with SWR1-C, or as reported here, in a role related to $\mathrm{RSC}-\mathrm{C}$ function. It is important to note that may of the genes involved play roles in chromatin remodelling related to endogenous gene expression. Chromatin 
remodelers such as Arp6 could affect fundamental processes such as transcription and translation and this should be further examined.

We also report that the role of ARP6 in NHEJ may be independent of INO80-C, despite physical interconnectivity with the complex (Figure 4.6), whose role in DSB repair is primarily in HR through recruitment of RPA and Rad51 in an MRX-dependent manner ${ }^{245}$. Instead we observe an ARP6 function related to the RSC-C complex and MRE11, important DSB first responders required for progression of the DNA damage response. Rsc2 is important for the recruitment of Tel1 and Mec1 to the site of damage and activation of Rad53 in the DNA damage checkpoint pathway ${ }^{93}$. The observed increase in repair fidelity (Figure 4.2) in ARP6 mutants may be related to the activity of MRX, potentially via MRX endonuclease activity, and the activation of Tel1, which are important for facilitating accurate repair ${ }^{97}$. Deletion of $R S C 2$ reduces the efficiency of NHEJ repair, possibly due to its physical interaction with Mre11 and Yku70 93,226,246, and this activity may also be influences by the presence/absence of Arp6. Additionally, loss of ARP6 leads to impaired HR (Figure 4.5) again suggesting that the ARP6's role in resolving breaks is likely not involved in the core NHEJ repair machinery but instead may be functioning in the early stages on DSB repair, particular in relaxation of chromatin structure around break sites. 


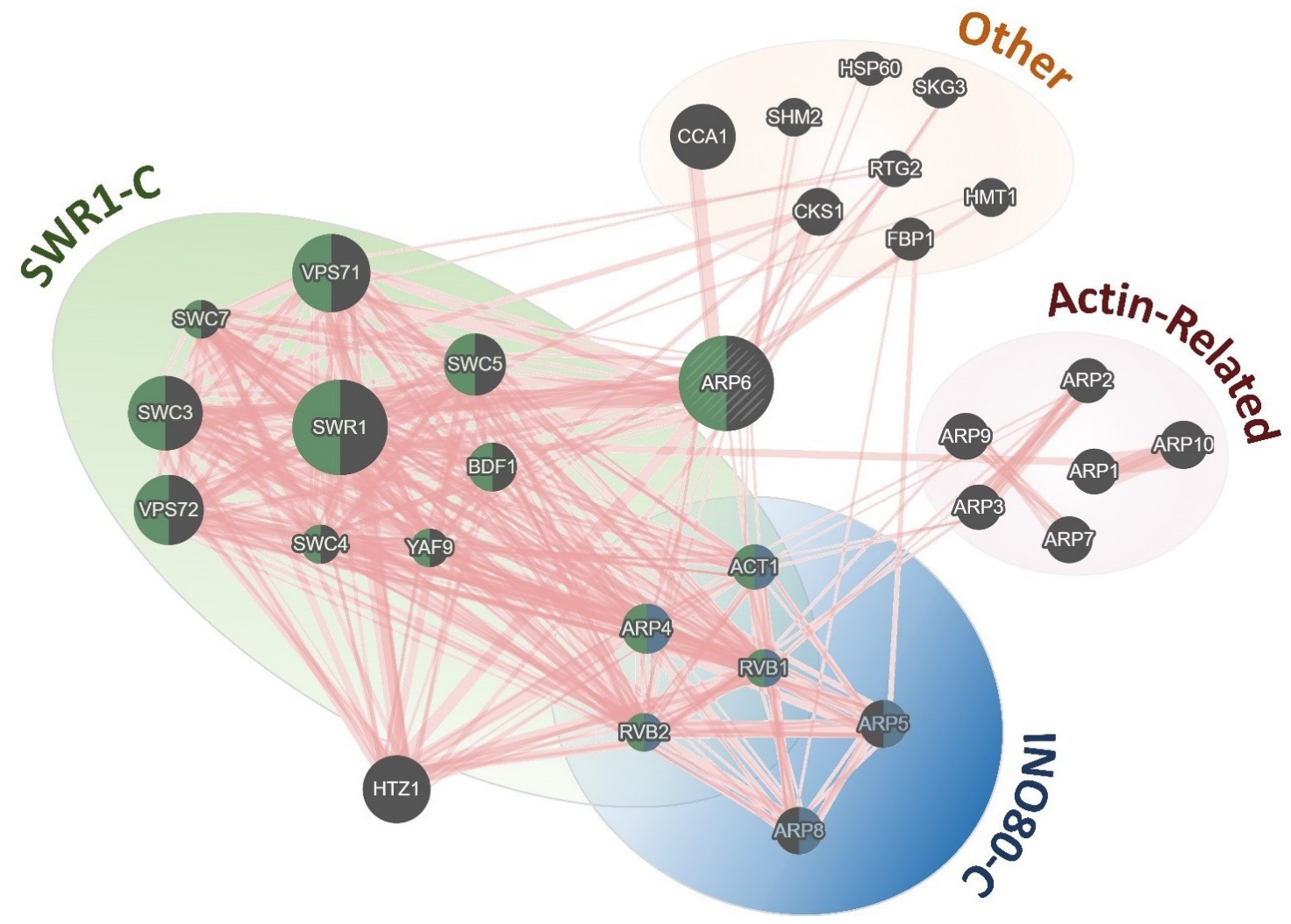

Figure 4.6: Protein-protein interaction network of Arp6 and associated proteins suggests functional associations with members of the SWR1-C and INO80-related chromatin remodeling complexes. Network produced using GeneMania (www.genemania.org). Nodes labelled with green are functionally-associated with the SWR1 complex and blue nodes indicate association with INO80-type complex (blue/green nodes are associated with both). Other genes are grouped into actin-related proteins (light red background) and other (light orange background).

\subsubsection{Summary and future directions}

In conclusion, NHEJ is an expansive and multifaceted process and it is important to identify all genes involved, from damage sensing, to repair and return to the cell cycle. This was the premise for our project which aimed to identify novel NHEJ repair 
genes. Molecular mechanisms underlying NHEJ in yeast continue to be used to understand how the pathway functions in human cells ${ }^{131,247}$. We report that $A R P 6$ is in fact a participant in NHEJ through MRE11 and the RSC-C complex activity. Additionally, this novel repair function is independent of SWR1-C activity in response to DNA damage. Additional biochemical investigations are needed to fully elucidate the association of Arp6 and Rsc2/Mre11 in the context of DNA repair. It is also interesting to examine whether the role of Arp6 in DNA repair is conserved in other organisms such as humans. 


\section{Chapter 5: RNAPII degradation factor Def1 influences NHEJ}

\subsection{Abstract}

Double strand breaks (DSBs) pose a serious risk to genetic integrity and must be repaired either by direct ligation through non-homologous end-joining (NHEJ) or through homologous recombination (HR). The Ku complex (Yku70/Yku80) plays a significant role in determining which pathway will be employed for repair. We tested a hypothesis that RNAPII degradation factor Def1 may influence pathway choice. Our results suggest that Def1 has limited influence on pathway choice but plays a significant role in responding to chromosomal double strand breaks despite binding Yku80 within a proposed regulatory domain. Interestingly, the overexpression of DEF1 rescues yku80 mutants from an impaired NHEJ phenotype.

\subsection{Introduction}

No other DNA lesion is as severe as a DSB. When a breaks occur, cells halt cell cycle progression until this structural lesion is resolved ${ }^{192}$. The causes of DSBs are numerous and include IR and UV radiation, chemical agents and endogenous sources including the production of ROS and collapsed replication forks ${ }^{92}$. DSBs can lead to mutations, chromosomal rearrangements, or apoptosis and must be repaired quickly and accurately to avoid these outcomes ${ }^{117}$. Eukaryotic cells have two competing pathways responsible for the repair of DSBs, homologous recombination (HR) utilizes sections of homologous DNA as a template in the facilitation of accurate repair and non- 
homologous end-joining which processes and directly ligates broken ends without requiring a template in an error-prone manner.

The choice of which repair pathway to employ considers a variety of factors including the availability of a homologous sequence and the current stage of the cell cycle. HR is the dominant mechanism in yeast but is most active during late $S$ phase and G2 while NHEJ is more likely to be employed during G1 but can be utilized at any stage of the cell cycle ${ }^{248}$. NHEJ is initiated following the binding of a YKU heterodimer (Yku70/Yku80) to both sides of the break. If YKU stays bound it will recruit additional repair factors such as Nej1, MRX and Dnl4-Lif1 ${ }^{123}$. The MRX complex works to bridge the gap between broken ends and ensure they remain in close proximity to one another. The ligation complex (Dn|4-Lif1) interacts with multiple factors including Nej1 and YKU while nucleases and polymerases process broken ends in preparation for ligation ${ }^{127}$. A break is only resolved via NHEJ if YKU remains bound and end resection is inhibited at the early stages following break detection. Interplay between YKU and end-resecting proteins including Exo1, Sae2 and Mre11 have a significant impact on the decision but regulatory mechanisms and chromatin remodeling activities also influence the choice $246,249,250$. A comprehensive understanding of the mechanism of pathway choice is still emerging and it is likely that additional factors/processes will be identified. Recently a new master regulator named CYREN was identified in human cells that inhibits NHEJ during S and G2 through binding and inhibition of the Ku80. Considering NHEJ is largely 
downregulated/inhibited in budding yeast, we posited that a similar mechanism may be at play in the yeast system.

The yeast RNA polymerase degradation factor Def1 plays a role in the degradation of RNA polymerase II which functions as a method of transcription regulation as well as a system for dissociating the polymerase from DNA should it encounter a DNA adduct ${ }^{251}$. Def1 is considered a DNA repair protein as also it promotes the ubiquitylation and degradation of the polymerase $\delta$ catalytic subunit Pol3 at stalled replication forks ${ }^{252}$. A systematic proteome-wide affinity purification/mass spectroscopy screen identified a physical interaction between Def1 and Yku80 ${ }^{64}$. We identified a region on Def1 that had high sequence similarity to the region on CYREN responsible for Ku binding. This work examines if Def1 influences repair pathway choice and if it is also able to bind Yku80 in a similar manner as CYREN interacts with Ku.

\subsection{Materials and Methods}

\subsubsection{Homologous recombination assay}

pGV-256/DEAD plasmid was digested at its non-functional lacZ gene with BgllI

232. Separately, a PCR-product containing functional lacZ was amplified using the pGV256/LIVE plasmid to provide a potential template for HR repair. 10-20 ng of 'dead' plasmid and $200 \mathrm{ng}$ of PCR product containing the functional lacZ cassette were cotransformed into mutant and WT strains and grown for 2 to 3 days on selective medium. Colonies were transferred to a new plate and grown for 1 day. A colony-lift assay was 
performed by transferring colonies onto a nitrocellulose membrane and lysing with liquid nitrogen, prior to incubation in Z-buffer containing X-gal (5-bromo-4-chloro-3indolyl- $\beta$-d-galactopyranoside) at $30^{\circ} \mathrm{C}$ for $\sim 4$ hours ${ }^{208}$. The frequency of blue colonies was normalized to the WT ratio to assess HR event frequency.

\subsubsection{Cell cycle assay}

WT and single mutants in a JKM139 background are incubated for 48 hours in YPD. These cells are then used to inoculate fresh cultures to and $\mathrm{OD}_{600}$ of $\sim 0.3-0.4$ in either YPD or YPD $+10 \mu \mathrm{g} / \mathrm{mL}$ of alpha factor and grown for 120 minutes. Cells are then washed three times with $\mathrm{dH}_{2} \mathrm{O}$ then serially diluted in $\mathrm{dH}_{2} \mathrm{O}$ and aliquots were plated on both YPD and YP + galactose solid medium then grown for 48 hours at $37^{\circ} \mathrm{C}$. The number of colony-forming units (CFUs) on galactose (damage induced) plates was divided by the number of CFUs on the YPD control plates then normalized to the WT JKM ratio.

\subsubsection{Protein purification and SPOT array analysis}

The yeast DEF1 gene was amplified using primers with flanking EcoRI and HindIII restriction sites off the Def1 BG1805 overexpression plasmid and sub-cloned into a pET28 overexpression vector. For purification, proteins were expressed in BL21 E.coli and were affinity purified using Ni-NTA agarose resin using a Bio-Rad gravity flow column according to manufacturer protocols. 
A SPOT array was constructed containing 18 amino acid long peptides spanning Def1 regions that are homologous to CYREN. Individual peptides were synthesized at 0.1-mmol scale on a Multipep RSi peptide synthesizer (Intavis Inc.) using standard Fmoc (N-(9-fluorenyl) methoxycarbonyl) chemistry. For fluorescein labeling, an appropriate amount of 5-(and-6)-carboxyfluorescein succinimidyl ester was added to a peptide resin, and the coupling reaction was allowed to proceed for $1 \mathrm{~h}$ at room temperature. Upon cleavage of a peptide from the resin using trifluoroacetic acid, the fluorescein-labeled peptide was separated from the unlabeled peptide by HPLC on a $\mathrm{C}_{18}$ column. Identities of the peptides were confirmed by mass spectrometry.

Peptide SPOT arrays were utilized as described previously ${ }^{174}$. All peptide SPOT arrays were blocked with 5\% BSA in TBST (0.1 M Tris- $\mathrm{HCl}, \mathrm{pH} 7.4,150 \mathrm{mM} \mathrm{NaCl}$, and $0.1 \%$ Tween 20 ) for $1 \mathrm{~h}$. Purified protein was added directly in the blocking buffer to a final concentration of $1 \mu \mathrm{M}$ and incubated with the SPOT array at room temperature for $1 \mathrm{~h}$. The array was then washed $3 \times 5$ min with TBST before a rabbit anti-His antibody (1:4000 dilution in TBST; Cat\# ab3553, abcam) was added. The membrane was allowed to incubate at room temperature for $30 \mathrm{~min}$ prior to $3 \times 5$-min washes with TBST. After final $3 \times 5$-min washes in TBST, the SPOT arrays were visualized by enhanced HRP-based chemiluminescence. 


\subsubsection{Compensation analysis}

JKM knockouts were constructed using homologous recombination. Def1 overexpression plasmid was transformed into various strains. Strains were grown to saturation in liquid YPD then washed and serially diluted in distilled water. Aliquots from the $10^{-1}-10^{-4}$ dilutions were spotted on solid YPD and YP + galactose and grown for 48 hours at $37^{\circ} \mathrm{C}$. Spots were visually analyzed and assigned a score (1-5) relative to the growth of the JKM WT. Four independent trials were completed and averaged. Results are presented as average score $+/$ - standard deviation.

\subsection{Results}

\subsubsection{Homologous recombination assay}

We assayed if the loss of DEF1 results in changes to the proportion of DSBs that are repaired via HR. In this assay, digested plasmid pGV256-DEAD which contains a nonfunctional beta-galactosidase gene is transformed with PCR product containing functional LacZ. Cells that repair the plasmid are selected for then screened for LacZ activity which would indicate that repair was performed via HR using the PCR product as a template. The loss of DEF1 did not result in a statistically significant change in the rate of HR (Figure 5.1). 


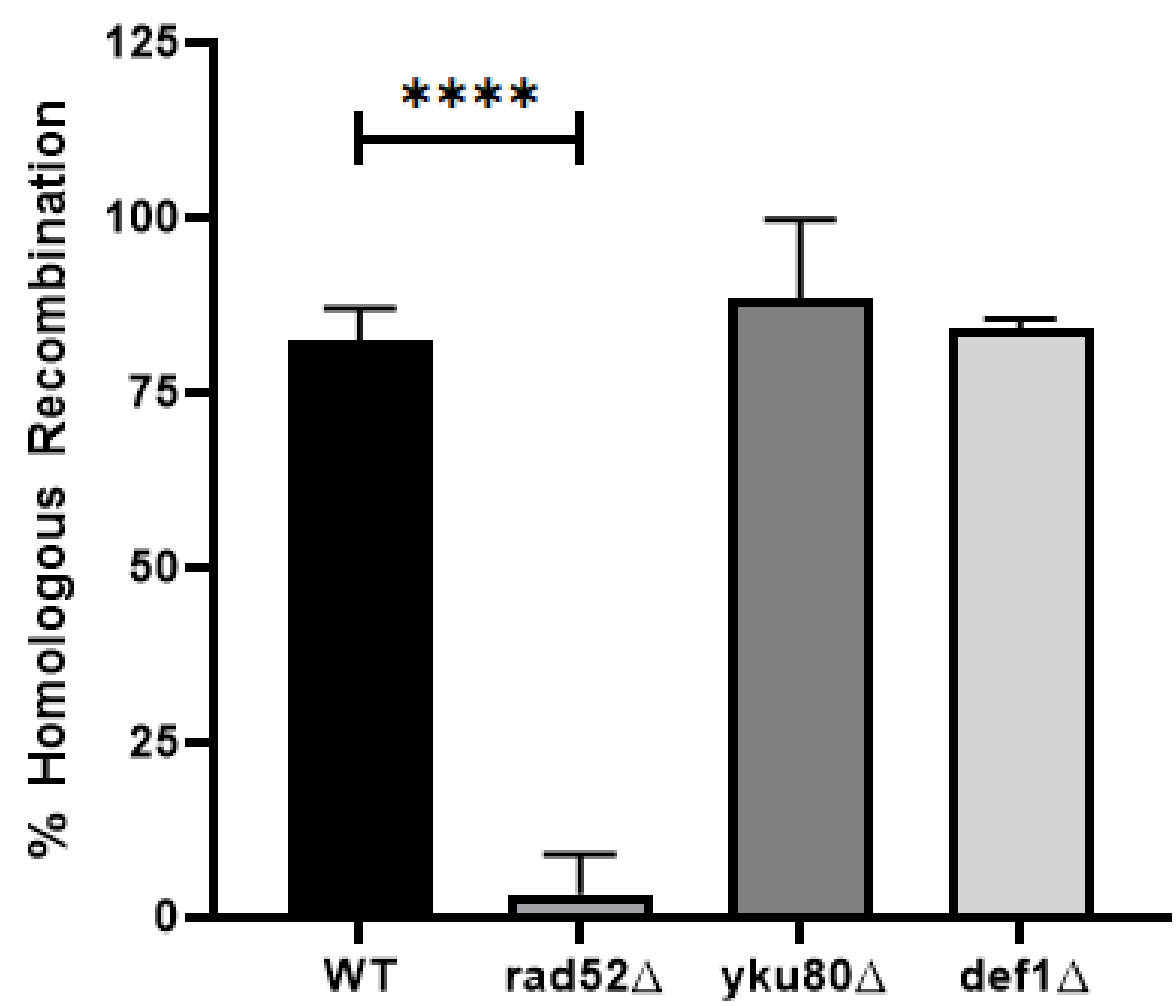

Figure 5.1: A plasmid based HR assay indicates that the loss of DEF1 does not significantly alter the rate of HR repair. Results indicate normalized rate of HR repair and were analyzed statistically using ANOVA (Dunnett's multiple comparison test).

DEF1 did not appear to significantly influence HR efficiency so we next examined if it influences NHEJ. We employed a JKM-based assay that continually induces chromosomal DSBs using an endogenous endonuclease that is expressed in the presence of galactose. Additionally, as NHEJ is known to be most active during G1, we added a second experimental set of synchronized cells. In the $\alpha$-factor condition, cultures were treated in the presence of $\alpha$-factor yeast pheromone which arrests cells in G1 and prevents transition into S phase. When treated cells are plated, they enter S phase as a synchronized culture as damage is induced. Figure 5.2 demonstrates that def1 $\Delta$ mutants dramatically reduce survivability under sustained DNA damage stress. 


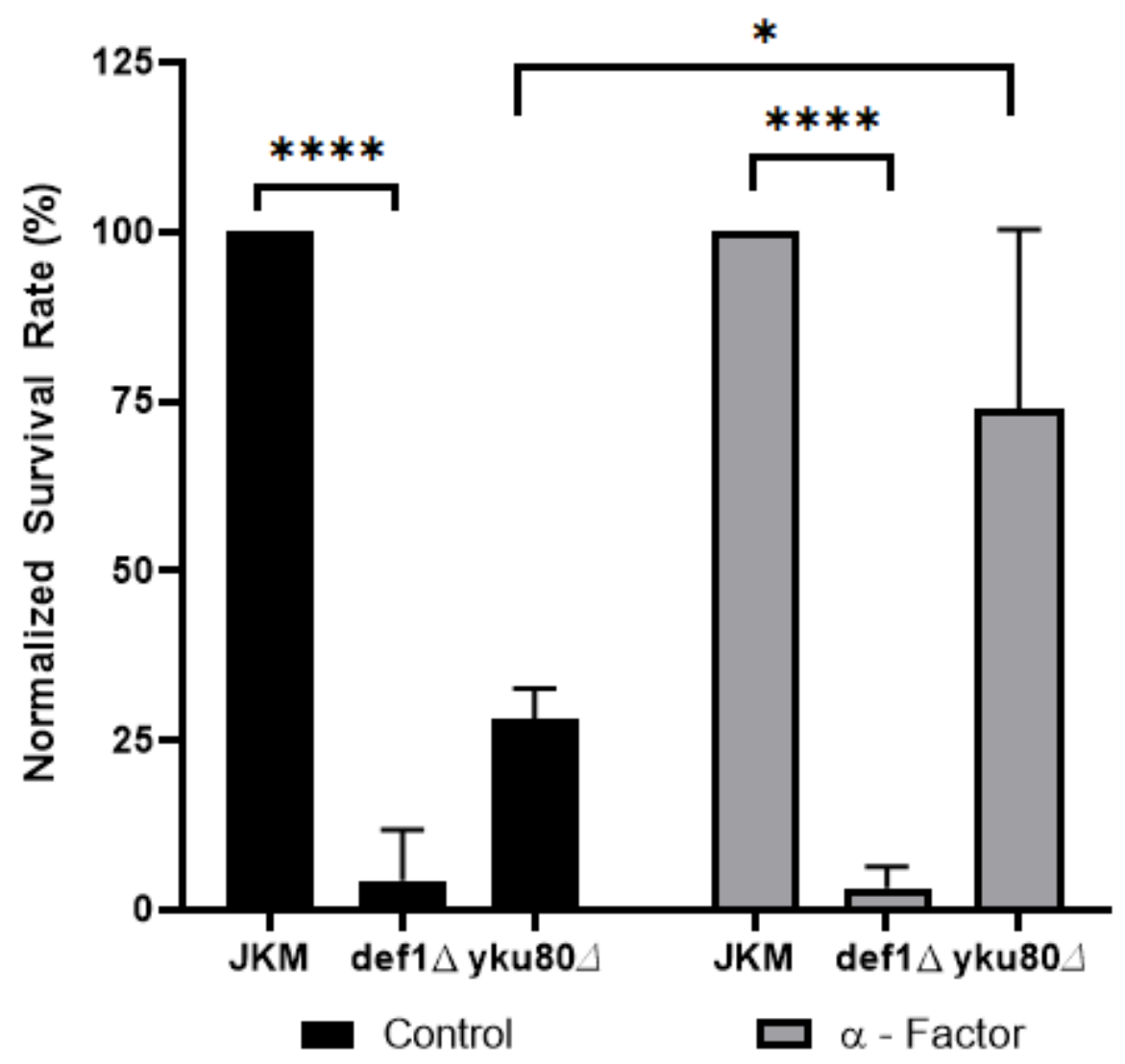

Figure 5.2 A chromosomal break assay in normal conditions and in synchronized cultures indicate that DEF1 has a significant role in DSB repair via NHEJ. Results show normalized survivability based on colony counts. Average of 3 trials is presented. Statistical analysis within a group was performed using one-way ANOVA and comparisons across groups was done by two-way ANOVA. If a $p$-value is less than 0.05 , it is flagged with one star $(*)$, if less than 0.01 it is flagged with two stars $\left({ }^{* *}\right)$ and if less than 0.001 , it is flagged with three stars $(* * *)$.

\subsection{Cell cycle assay}

The loss of DEF1 resulted in normalized repair rates of $4.3 \%$ and $3.3 \%$ compared to WT in the normal and synchronized conditions respectively. In many cases, def1 $\Delta$ experimental plates did not produce a single viable colony despite increasing the 
number of cells plated. Microcolonies, difficult to detect with the human eye formed but did not proliferate beyond this early stage. The Yku80 control has a significant increase in repair efficiency in the synchronized culture. This result indicates that DEF1 is a very important NHEJ repair factor. To understand if this influence on DSB repair is related to an interaction with Yku80, we performed a peptide binding assay that will determine if Def1 interacts with YKU in a region with high similarity to CYREN (Figure 5.3).

\subsection{Spot array indicates binding between Def1 and Yku80}

A

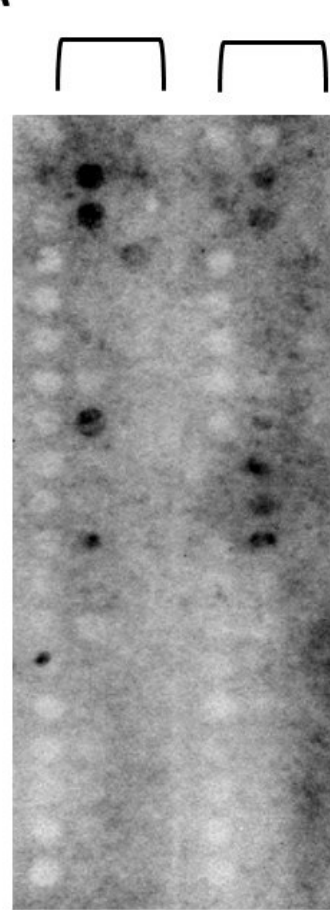

$\mathrm{N}=2$
B

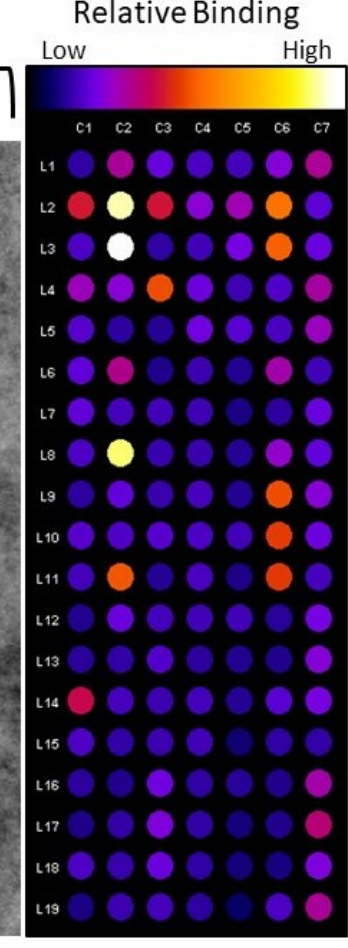
High

C

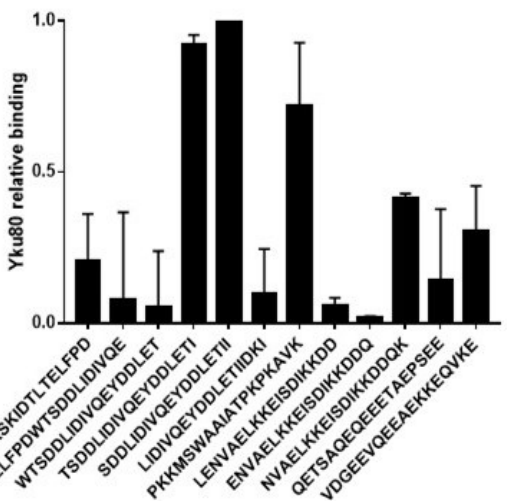

\begin{tabular}{|c|c|c|c|}
\hline Peptide Sequence & Loci & Region & Relative Binding \\
\hline SDDLIDIVQEYDDLEIII & $37-55$ & Predicted Interaction & 1 \\
\hline TSDDLIDIVQEYDDLETI & $36-54$ & Predicted Interaction & 0.923 \\
\hline PKKMSWAAIATPKPKAVK & $239-256$ & Predicted Interaction & 0.72 \\
\hline NVAELKKEISDIKKDDQK & $264-281$ & Predicted Interaction & 0.411 \\
\hline LNPALKSKIDTLTELFPD & $19-36$ & Predicted Interaction & 0.204 \\
\hline LIDIVQEYDDLEIIIDKI & $42-60$ & Predicted Interaction & 0.097 \\
\hline TELFPDWTSDDLIDIVQE & $31-48$ & Predicted Interaction & 0.078 \\
\hline LENVAELKKEISDIKKDD & $262-279$ & Predicted Interaction & 0.06 \\
\hline WTSDDLIDIVQEYDDLET & $35-53$ & Predicted Interaction & 0.051 \\
\hline ENVAELKKEISDIKKDDQ & $263-280$ & Predicted Interaction & 0.018 \\
\hline QETSAQEQEEETAEPSEE & $274-291$ & Homologous Region & 0.143 \\
\hline VDGEEVQEEAEKKEQVKE & $316-333$ & Homologous Region & 0.306 \\
\hline
\end{tabular}

Figure 5.3: A peptide binding array indicates that Def1 interacts with Yku80 in a region with high sequence similarity to CYREN. A) Demonstrate results from $2 X$ SPOT binding 
probes with relative binding shown. B-C) Shows relative binding scores and sequences of 14 peptides representing Def1 motifs that bound Yku80.

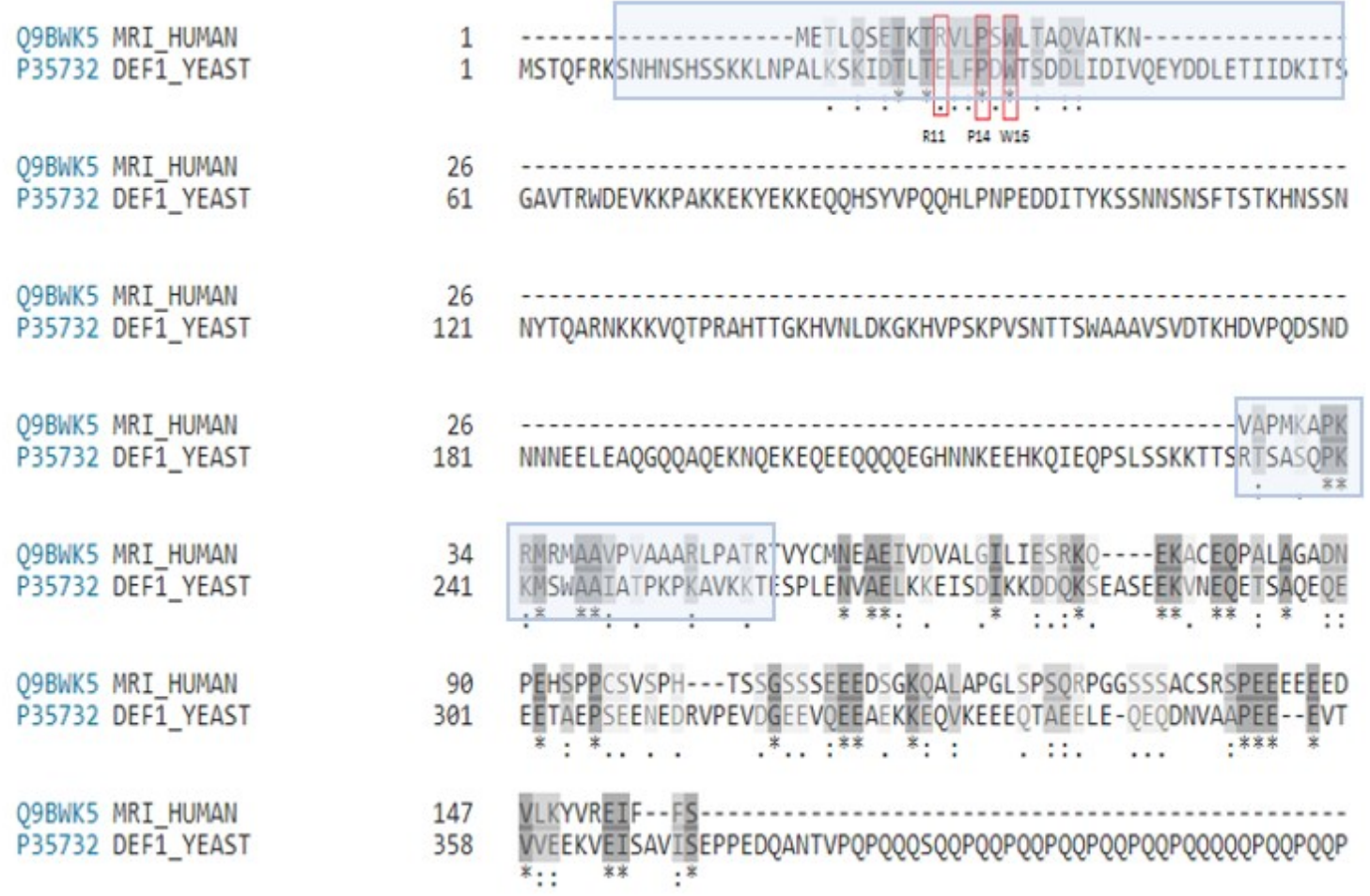

Figure 5.4: Alignment of Def1 and CYREN using BlastP. Predicted interaction sites based on the reported CYREN interaction motifs are shown in the blue boxes. Highly conserved residues across the animal kingdom are shown in the red boxes.

Arnoult et al, 2017 performed a very similar SPOT binding assay that indicated three regions in close proximity that demonstrated binding to YKU80 ${ }^{253}$. Our findings indicated that binding between yeast Def1 and Yku 80 significantly overlaps two out of three homologous regions (Table 5.1) 
Table 5.1: Summary of Def1 binding. Three interacting regions were previously identified on CYREN. We probed the corresponding regions on Def1. 2/3 interaction sites overlapped with the reported CYREN binding sites.

\begin{tabular}{lllll}
\hline $\begin{array}{l}\text { Interaction } \\
\text { Site }\end{array}$ & Def1 Region & $\begin{array}{l}\text { Corresponding } \\
\text { Region } \\
\text { CYREN }\end{array}$ & $\begin{array}{l}\text { Reported Cyren } \\
\text { Interaction Sites }\end{array}$ & \% Overlap \\
\hline $\mathbf{1}$ & $19-36$ & $-3-14$ & $8-28$ & $30 \%$ \\
& $35-53$ & $16-25$ & $8-28$ & $100 \%$ \\
\hline $\mathbf{2}$ & $239-256$ & $32-48$ & $31-50$ & $100 \%$ \\
\hline $\mathbf{3}$ & $262-280$ & $55-73$ & N/A & $0 \%$ \\
\hline
\end{tabular}

\subsubsection{Genetic compensation}

A spot test was used to study if DEF1 overexpression is able to reduce NHEJ repair

efficiency (Figure 5.5). Our original hypothesis that Def1 may be functioning as a inhibitor of Yku80 was not supported in that if this mechanism was present, overexpression of DEF1 should theoretically decrease NHEJ efficiency. However, we identified a very interesting relationship in that the overexpression of DEF1 in a $y k u 80 \triangle$ background fully recovered the mutant's reduced ability to repair chromosomal breaks. 


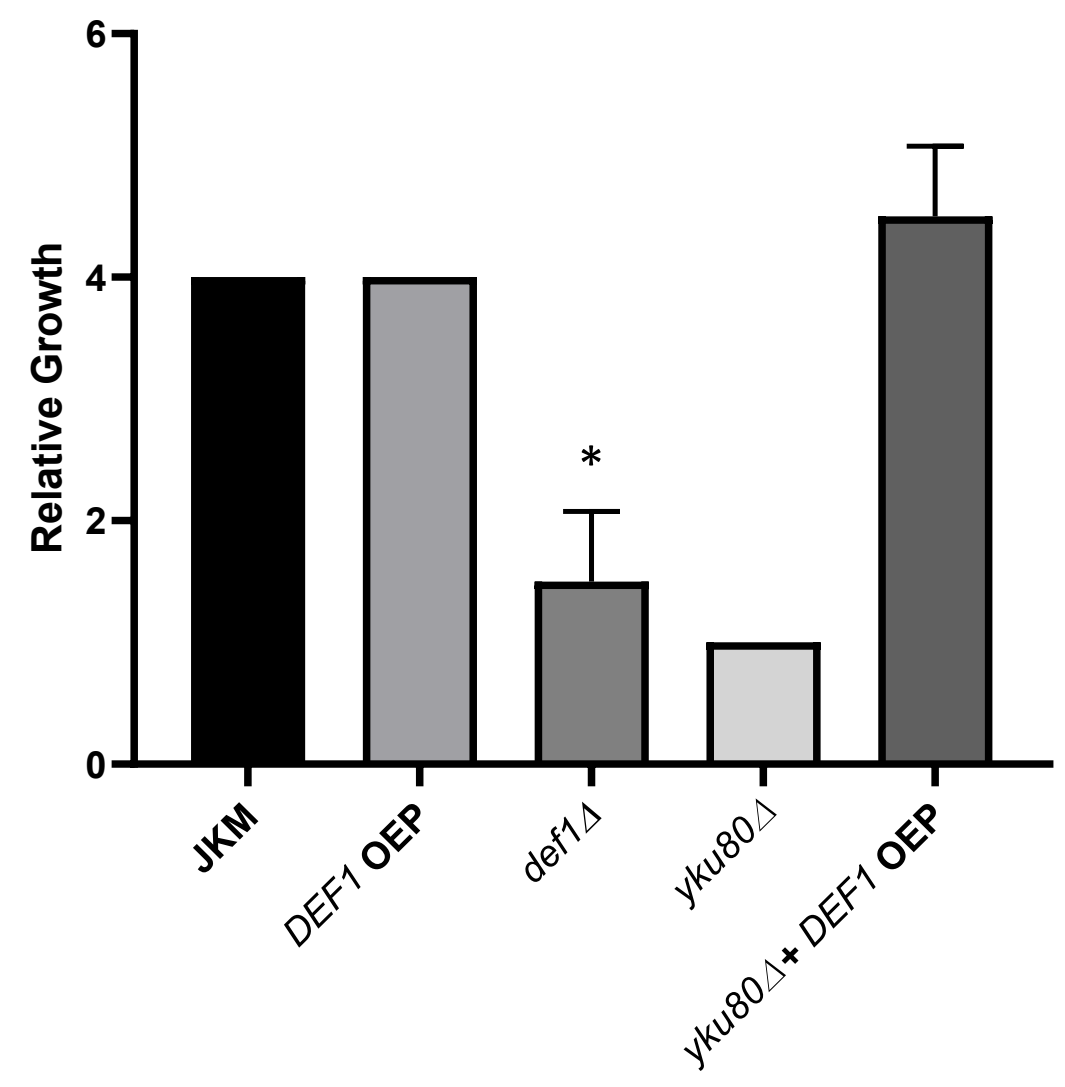

Figure 5.5: Overexpression of DEF1 does not alter the ability to repair chromosomal breaks. Results from this chromosomal break assay indicate that overexpression of DEF1 rescues the $y k u 80 \Delta$. This figure shows the combined results of four independent spot tests that were visually scored (1: minimal growth -5 : growth beyond WT growth rate). Average scores +/- standard deviation are presented.

Together these results suggest that although Def1 does not appear to be inhibiting Yku80 function, it is an important factor in NHEJ repair.

\subsection{Discussion}

This project probed a possible role for Def1 in yeast NHEJ. Particularly, we first studied if Def1 influences DSB repair pathway choice. Because of the strong homology to the YKU80 interacting region on CYREN, it was possible that a similar mechanism of 
inhibition could be seen in yeast. However, the results in Figure 5.1 indicate that the loss of DEF1 does not decrease the rate of HR. Additionally, the overexpression of DEF1 did not result in a decrease in the ability to repair severed chromosomes (Figure 5.4). Of note, the loss of $Y K U 80$, one of the most important NHEJ repair proteins, did also not significantly change the rate of HR repair (Figure 5.1). This may indicate that the assay primarily detects only genes that significantly influence the HR pathway such as Rad52.

Based on these findings we can conclude that although Def1 binds Yku80 within the same region as CYREN (Figure 5.3), it does not have the same inhibitory capacity as reported for CYREN. Despite strong sequence similarity in the proposed binding region (Figure 5.4), there are notable differences between the two proteins. Particularly, CYREN is a small protein (145aa) while Def1 is considerably larger at 745aa. Additionally, the proposed interaction site(s) are discontinuous compared to CYREN - with a 200aa gap in between the homologous regions (Figure 5.4). This proposed Yku regulatory site may theoretically be bound by a variety of activating or inhibitory proteins or proteins result in no change. The cell cycle assay indicated that in both the control and synchronized population, def1 $\Delta$ mutants were unable to recover from persistent chromosomal breaks (Figure 5.2). This severe phenotype prevented a more thorough analysis of $D E F 1$ 's role in cell cycle dynamics.

We did however provide support for the role of Def1 as a NHEJ repair factor and suggested that it may have additional functions in the repair pathway to those that are already known. Def1 is known to be important for degrading Pol3 at stalled replication 
forks, a mechanism that prevents the development of DSBs that would likely be resolved by $H R{ }^{107,252}$. The assays that were employed here should theoretically bypass this mechanism as they are designed to induce a double strand break that must be directly ligated by NHEJ because homologous regions have been removed. Def1 could potentially be influencing NHEJ through Pol4, the polymerase responsible for gap filling during NHEJ end processing ${ }^{127}$. If Def1 targets Pol4 for degradation, Def1 could potentially be important for removing Pol4 from break sites once gap filling is complete.

Another very important finding is that the overexpression of DEF1 is able to suppress yku80 $\Delta^{\prime}$ s severe impaired ability to repair broken chromosomes. This is unique as there are limited explanations as to how breaks are directly ligated without YKU. Yeast can repair DSBs through a YKU independent pathway but this type of repair results in high rates of mutations ${ }^{254}$. Often deemed alternative NHEJ or microhomology mediated end-joining, this backup pathway can function when conventional repair mechanisms are unavailable to rejoin ends by using short 5-25 NT regions of homology 255,256 . It is possible that the overexpression of DEF1 is potentially driving alternativeNHEJ.

The results here provide evidence that Def1 may play multiple roles in NHEJ. The Def1/Yku80 interaction was only previously reported in a single large-scale study but we support this finding here. A potential model suggested is that Def1 binds YKU at DSB sites and functions to promote degradation of Pol4 following the completion of gap- 
filling. Further biochemical assays are required to provide support for this model but support provided herein is intriguing. 


\section{Chapter 6: Conclusions}

\subsection{Concluding Remarks}

The field of systems biology is increasingly becoming synonymous with the term "big data" and molecular biologists now must be adept at navigating exhaustive datasets in the search for novel gene functions or meaningful protein interactions. While researchers become increasingly more capable of handling expanding datasets and computation power increases, some fields in systems biology such as de novo protein design still remain in their infancy while other more established fields such as functional genomics are highly dynamic due to the overwhelming complexity of attempting to study life on a systems-level scale.

Bioinformatics uses computational tools to interpret existing data to infer novel insights, and to train on available data to predict biologically relevant functions or engineering new biological molecules. Here we have successfully employed all these different categories of tools to predict novel gene functions, infer functional relationships, and engineer novel synthetic biological molecules. The explosive growth of omics data has generated new challenges that require researchers to regularly innovate to keep pace with the scale of available data ${ }^{257}$.

In the face of comprehensive omics-level research projects reporting hundreds or thousands of novel claims in a single manuscript, it can be daunting searching for new insights in an era where data production significantly outpaces the ability to adequately 
interpret new findings. However, finding a needle in haystack remains possible and applying unique approaches can allow one to identify novel protein functions even in remarkably well-characterized species such a S. cerevisiae. Despite nearly twenty years of large scale screens aimed at identifying new repair genes/gene functions ${ }^{131,237,258-260 \text {, }}$ novel discoveries into eukaryotic DNA repair mechanisms continue to occur using the yeast model system.

The NHEJ pathway model in particular continues to evolve regularly as new components/mechanisms are identified ${ }^{132,233,261}$. In yeast, NHEJ is a relatively inconsequential pathway considering HR dominates and YKU7O mutants are viable ${ }^{27}$. The reasons for the continued interest in studying the pathway stem primarily from the potential applications of the findings more so than a fundamental interest in studying the underlying biology. NHEJ is implicated in genetic diseases, cancer, aging, immunology and new genomic editing techniques. New findings in NHEJ mechanisms have the potential to impact applications in any of these diverse fields.

Impaired NHEJ can result in genetic diseases such ataxia telangiectasia and primordial dwarfism ${ }^{262}$ but NHEJ is more actively studied for its relationship to cancer. Defects in NHEJ can lead to chromosomal instability that is a hallmark of cancer ${ }^{263}$ but NHEJ repair factors are also an attractive target for cancer therapeutics and the identification of new factors may also translate into the identification of a novel targets ${ }^{264}$. For example, DNA ligase IV is an attractive therapeutic target and the recently developed inhibitor Na-SCR7-P, a more water soluble version of a previously identified 
ligase inhibitor, is capable of preventing ligation during end joining resulting in increased rates of apoptosis and decreased tumor growth in the later stages of treatment ${ }^{265}$. Mutations that impair NHEJ have a general detrimental effect in cell survivability which is a major driver of aging ${ }^{266}$. NHEJ also underlies the process by which B-cells and T-cells generate antigen receptors through VDJ recombination which utilizes the variable, diversity, and joining genes. Mutations in the NHEJ1 gene, homolog of Nej1, result can cause an immunodeficiency (Cernunnos/XLF deficiency) disease and defects in additional repair factors such as ATM, 53PBP1, or H2AX can cause severe disease complications. Mutations in genes encoding for core human NHEJ factors Lig4, Cernunnos, DNA PKCs, Artemis and XRCC4 have all been associated with severe combined immunodeficiency (SCID) ${ }^{89}$. In addition to all these reasons for studying NHEJ, the most pertinent application for the pathway currently its involvement in genetic editing using the CRISPR-Cas9 system.

The CRISPR-Cas9 system is an excited new methodology for genetic engineering that may allow for highly efficient editing of multiple cells simultaneously in vivo. However, despite the initial hype behind the approach, the technology has suffered from complications that arise when Cas-9 induces a DSB during the editing process. The inherent inaccuracy and variability in NHEJ is not always seen in repair following cleavage by Cas 9 which can be surprisingly accurate when ligatable ends are produced 267. This property can be exploited to improve genome editing by CRISPR by engineering breaks that are repaired by accurate NHEJ ${ }^{267}$. In other CRISPR applications, mutations 
introduced through NHEJ impair functionality of the system. For example, Cas9 in association with T-cells can be guided to cleave proviral HIV DNA and profoundly inhibit viral replication short term ${ }^{268}$. Mutations caused by NHEJ repair of the Cas9 cleavage site including insertions, deletions and substitutions allowed the virus to quickly evolve resistance to this therapy. A more comprehensive understanding of the NHEJ mechanism and new methods of modulating NHEJ could greatly assist in efficient design of effective CRISPR systems.

In this thesis, three components of the NHEJ pathway have been studied. These results could have potential implications in these systems where the pathway is associated with important applications. Our group has identified a variety of novel NHEJ components in the past including histone acetyl transferase RTT109 and interacting partner Vps75, spindle checkpoint factors Bub1 and Bub2 that promote NHEJ and mitotic exit, and repair regulators Pph3/Psy2 among others 131,207,208. Each time a new component is added to the evolving model, it opens opportunities for discovering even more interacting factors that may not have been implicated if the other factors were not first identified.

PSK1 and its interaction with RAD27 may help to solidify Rad27 as a core NHEJ factor and help to explain unresolved regulatory mechanisms responsible for modulating DSB repair. Psk1 is a kinase that becomes more active during stress conditions and may well have additional yet to be identified targets within the repair process. Chromatin remodeling was heavily studied in the past for its role in DSB repair, 
but this field has been relatively dormant lately. Introducing a proposed model for ARP6 function in relation to central chromatin remodeling complexes may help to clarify existing models and identify important relationships. Def1, although previously associated with DNA repair, must be further examined to understand the effect its binding has on Ku activity. The finding reported here that overexpression of DEF1 can functionally compensate for YKU8O in NHEJ is significant as current models do not propose redundancy in the essential Ku toolbelt protein and instead suggest driving alternative-NHEJ.

\subsection{Future Directions}

Here we both developed and employed systems biology approaches to produce valuable novel findings and pioneering synthetic biology tools. The development of InSiPS as a new tool for designing binding proteins that is truly unique from anything else currently available. Given our preliminary demonstration of the effectiveness of InSiPS and the great potential of the tool, there exists boundless opportunity to target proteins of interest in different species. Future iterations of InSiPS should include additional considerations for predicted protein stability and 3D structure to avoid designing proteins that are unstable or have an unavailable binding motif. I suspect this novel tool will drive new technologies that improve our abilities to design novel proteins. 
We used systems biology tools to identify three new participants in the NHEJ pathway. However, each of the proteins studied in this thesis will need to be subjected to further biochemical analysis to understand specific molecular functions and modulate the current NHEJ model accordingly. These proteins were identified by analyzing data from proteome-wide screens for PPIs, both from the benchtop and from computation models. Once identified, I used other systems-biology tools to generate hypotheses and infer mechanisms. This skill is highly applicable to many different fields of research and research questions. Science is constantly evolving and trends change quickly but I suspect that an ability to infer novel insights by applying the top-down/bottom-up approach will prove highly adaptable no matter the direction taken for some time to come. The ability to identify interesting molecular mechanisms by probing massive genomic/proteomic/transcriptomic datasets, design and perform experiments to test hypotheses, and interpret findings within a larger systems context is a skill that should serve any scientist well moving forward. I hope that the findings here make a meaningful contribution to the quickly evolving field of systems biology. 


\section{References}

1. Kirschner MW. The meaning of systems biology. Cell. 2005;121(4):503-504. doi:10.1016/j.cell.2005.05.005

2. Nielsen J. Systems Biology of Metabolism: A Driver for Developing Personalized and Precision Medicine. Cell Metab. 2017;25(3):572-579.

doi:10.1016/j.cmet.2017.02.002

3. Gu Z, Eils R, Schlesner M. Complex heatmaps reveal patterns and correlations in multidimensional genomic data. Bioinformatics. 2016;32(18):2847-2849. doi:10.1093/bioinformatics/btw313

4. Vizcaíno JA, Deutsch EW, Wang R, et al. ProteomeXchange provides globally coordinated proteomics data submission and dissemination. Nat Biotechnol. 2014;32(3):223-226. doi:10.1038/nbt.2839

5. Hashemifar S, Ma J, Naveed H, Canzar S, Xu J. ModuleAlign: module-based global alignment of protein-protein interaction networks. Bioinformatics. 2016;32(17):i658-i664. doi:10.1093/bioinformatics/btw447

6. Tyanova S, Temu T, Sinitcyn P, et al. The Perseus Computational Platform for 
Comprehensive Analysis of (Prote)Omics Data. Vol 13. Nature Publishing Group; 2016:731-740. doi:10.1038/nmeth.3901

7. Siuzdak G, Patti GJ, Yanes O. Innovation: Metabolomics: the apogee of the omics trilogy. Nat Rev Mol Cell Biol. 2012;13(4):263-269.

8. Gusev A, Ko A, Shi H, et al. Integrative approaches for large-scale transcriptomewide association studies. Nat Genet. 2016;48(3):245-252. doi:10.1038/ng.3506

9. Ritchie MD, Holzinger ER, Li R, Pendergrass SA, Kim D. Methods of integrating data to uncover genotype-phenotype interactions. Nat Rev Genet. 2015;16(2):8597. doi:10.1038/nrg3868

10. Zhang Z, Zhao W, Xiao J, et al. The BIG Data Center: From deposition to integration to translation. Nucleic Acids Res. 2017;45(D1):D18-D24. doi:10.1093/nar/gkw1060

11. Marx V. The big challenges of big data. Nature. 2013;498(7453):255-260. doi:10.1038/498255a

12. Park CY, Wong AK, Greene CS, et al. Functional Knowledge Transfer for Highaccuracy Prediction of Under-studied Biological Processes. Tucker-Kellogg G, ed. PLoS Comput Biol. 2013;9(3):e1002957. doi:10.1371/journal.pcbi.1002957

13. Korneliussen TS, Albrechtsen A, Nielsen R. ANGSD: Analysis of Next Generation Sequencing Data. BMC Bioinformatics. 2014;15(1):356. doi:10.1186/s12859-014- 
0356-4

14. Priest NK, Rudkin JK, Feil EJ, et al. From genotype to phenotype: can systems biology be used to predict Staphylococcus aureus virulence? Nat Rev Microbiol. 2012;10(11):791-797. doi:10.1038/nrmicro2880

15. Bandyopadhyay S, Mehta M, Kuo D, et al. Rewiring of genetic networks in response to DNA damage. Science (80- ). 2010;330(6009):1385-1389. doi:10.1126/science.1195618

16. Costanzo M, VanderSluis B, Koch EN, et al. A global genetic interaction network maps a wiring diagram of cellular function. Science. 2016;353(6306):aaf1420. doi:10.1126/science.aaf1420

17. Gavin A-C, Bösche M, Krause R, et al. Functional organization of the yeast proteome by systematic analysis of protein complexes. Nature. 2002;415(6868):141-147. doi:10.1038/415141a

18. Bunnik EM, Roch KG Le. An Introduction to Functional Genomics and Systems Biology. Adv Wound Care. 2013;2(9):490. doi:10.1089/WOUND.2012.0379

19. Mattiazzi M, Petrovič U, Križaj I. Yeast as a model eukaryote in toxinology: A functional genomics approach to studying the molecular basis of action of pharmacologically active molecules. Toxicon. 2012;60(4):558-571. doi:10.1016/J.TOXICON.2012.03.014 
20. Lopes H, Rocha I. Genome-scale modeling of yeast: chronology, applications and critical perspectives. FEMS Yeast Res. 2017. doi:10.1093/femsyr/fox050

21. Kim C, Yang J, Jeong SH, et al. Yeast-based assays for characterization of the functional effects of single nucleotide polymorphisms in human DNA repair genes. PLoS One. 2018. doi:10.1371/journal.pone.0193823

22. Taylor EM, Lehmann AR. Conservation of eukaryotic DNA repair mechanisms. Int J Radiat Biol. 1998;74(3):277-286. http://www.ncbi.nlm.nih.gov/pubmed/9737531. Accessed July 17, 2019.

23. Foltman M, Molist I, Sanchez-Diaz A. Synchronization of the Budding Yeast Saccharomyces cerevisiae. In: ; 2016:279-291. doi:10.1007/978-1-4939-31453_19

24. Rego A, Trindade D, Chaves SR, et al. The yeast model system as a tool towards the understanding of apoptosis regulation by sphingolipids. FEMS Yeast Res. 2014;14(6):995-995. doi:10.1111/1567-1364.12191

25. Goffeau AA, Barrell BG, Bussey H, et al. Life with 6000 genes. Science (80- ). 1996;274(5287):546-567. doi:10.1126/science.274.5287.546

26. Giaever G, Nislow C. The yeast deletion collection: A decade of functional genomics. Genetics. 2014. doi:10.1534/genetics.114.161620

27. Giaever G, Chu AM, Ni L, et al. Functional profiling of the Saccharomyces 
cerevisiae genome. Nature. 2002. doi:10.1038/nature00935

28. Z. L, F.J. V, S. B, et al. Systematic exploration of essential yeast gene function with temperature-sensitive mutants. Nat Biotechnol. 2011;29(4):361-367. https://www.nature.com/nbt/journal/v29/n4/abs/nbt.1832.html. Accessed January 30, 2019.

29. Jones GM, Stalker J, Humphray S, et al. A systematic library for comprehensive overexpression screens in Saccharomyces cerevisiae. Nat Methods. 2008;5(3):239-241. doi:10.1038/nmeth.1181

30. Galván Márquez I, Akuaku J, Cruz I, Cheetham J, Golshani A, Smith ML. Disruption of protein synthesis as antifungal mode of action by chitosan. Int J Food Microbiol. 2013;164(1):108-112. doi:10.1016/J.IJFOODMICRO.2013.03.025

31. Samanfar B, Shostak K, Moteshareie H, et al. The sensitivity of the yeast, Saccharomyces cerevisiae, to acetic acid is influenced by DOM34 and RPL36A. PeerJ. 2017;5:e4037. doi:10.7717/peerj.4037

32. Hoepfner D, Helliwell SB, Sadlish H, et al. High-resolution chemical dissection of a model eukaryote reveals targets, pathways and gene functions. Microbiol Res. 2014. doi:10.1016/j.micres.2013.11.004

33. Guénolé A, Srivas R, Vreeken K, et al. Dissection of DNA Damage Responses Using Multiconditional Genetic Interaction Maps. Mol Cell. 2013;49(2):346-358. doi:10.1016/j.molcel.2012.11.023 
34. Lu P, Vogel C, Wang R, Yao X, Marcotte EM. Absolute protein expression profiling estimates the relative contributions of transcriptional and translational regulation. Nat Biotechnol. 2007;25(1):117-124. doi:10.1038/nbt1270

35. Huh W-K, Falvo J V., Gerke LC, et al. Global analysis of protein localization in budding yeast. Nature. 2003;425(6959):686-691. doi:10.1038/nature02026

36. Krogan NJ, Cagney G, Yu H, et al. Global landscape of protein complexes in the yeast Saccharomyces cerevisiae. Nature. 2006;440(7084):637-643. doi:10.1038/nature04670

37. Jewison T, Knox C, Neveu V, et al. YMDB: the Yeast Metabolome Database. Nucleic Acids Res. 2012;40(D1):D815-D820. doi:10.1093/nar/gkr916

38. Engel S, Balakrishnan R, ... GB-N acids, 2009 U. Saccharomyces Genome Database provides mutant phenotype data. academic.oup.com. https://academic.oup.com/nar/article-abstract/38/suppl_1/D433/3112267.

39. Hodges PE, McKee AHZ, Davis BP, Payne WE, Garrels Jl. The Yeast Proteome Database (YPD): A model for the organization and presentation of genome-wide functional data. Nucleic Acids Res. 1999;27(1):69-73. doi:10.1093/nar/27.1.69

40. Roth FP, Lipshitz HD, Andrews BJ. Q\&amp;A: Epistasis. J Biol. 2009;8(4):35. doi:10.1186/jbiol144

41. Zhang J. Epistasis Analysis Goes Genome-Wide. Gojobori T, ed. PLOS Genet. 
2017;13(2):e1006558. doi:10.1371/journal.pgen.1006558

42. Mani R, St.Onge RP, Hartman JL, Giaever G, Roth FP. Defining genetic interaction. Proc Natl Acad Sci. 2008;105(9):3461-3466. doi:10.1073/pnas.0712255105

43. Costanzo M, Baryshnikova A, Myers CL, Andrews B, Boone C. Charting the genetic interaction map of a cell. Curr Opin Biotechnol. 2011;22(1):66-74. doi:10.1016/J.COPBIO.2010.11.001

44. Burnside D, Moteshareie H, Marquez IG, et al. Use of chemical genomics to investigate the mechanism of action for inhibitory bioactive natural compounds. In: Bioactive Natural Products: Chemistry and Biology. ; 2015. doi:10.1002/9783527684403.ch2

45. Falconer SB, Czarny TL, Brown ED. Antibiotics as probes of biological complexity. Nat Chem Biol. 2011. doi:10.1038/nchembio.590

46. Collins SR, Miller KM, Maas NL, et al. Functional dissection of protein complexes involved in yeast chromosome biology using a genetic interaction map. Nature. 2007;446(7137):806-810. doi:10.1038/nature05649

47. Acuner Ozbabacan SE, Engin HB, Gursoy A, Keskin O. Transient protein-protein interactions. Protein Eng Des Sel. 2011;24(9):635-648. doi:10.1093/protein/gzr025

48. Chica C, Diella F, Gibson TJ. Evidence for the Concerted Evolution between Short 
Linear Protein Motifs and Their Flanking Regions. PLoS One. 2009;4(7).

doi:10.1371/journal.pone.0006052

49. Davey NE, Van Roey K, Weatheritt RJ, et al. Attributes of short linear motifs. Mol Biosyst. 2012;8(1):268-281. doi:10.1039/c1mb05231d

50. Schoenrock A, Samanfar B, Pitre S, et al. Efficient prediction of human proteinprotein interactions at a global scale. BMC Bioinformatics. 2014;15(1):1-22. doi:10.1186/s12859-014-0383-1

51. Szklarczyk D, Franceschini A, Wyder S, et al. STRING v10: protein-protein interaction networks, integrated over the tree of life. Nucleic Acids Res. 2015;43(D1):D447-D452. doi:10.1093/nar/gku1003

52. Feiglin A, Moult J, Lee B, Ofran Y, Unger R. Neighbor Overlap Is Enriched in the Yeast Interaction Network: Analysis and Implications. Tramontano A, ed. PLoS One. 2012;7(6):e39662. doi:10.1371/journal.pone.0039662

53. von Mering C, Krause R, Snel B, et al. Comparative assessment of large-scale data sets of protein-protein interactions. 2002;417(6887):399-403. doi:10.1038/nature750

54. Fields S, Song O. A novel genetic system to detect protein-protein interactions. Nature. 1989;340(6230):245-246. doi:10.1038/340245a0

55. Brückner A, Polge C, Lentze N, Auerbach D, Schlattner U. Yeast Two-Hybrid, a 
Powerful Tool for Systems Biology. Int J Mol Sci. 2009;10:2763-2788.

doi:10.3390/ijms10062763

56. $\mathrm{Yu} \mathrm{H}$, Braun $\mathrm{P}$, Yildirim MA, et al. High-Quality Binary Protein Interaction Map of the Yeast Interactome Network. Science (80- ). 2008;322(5898):104-110. doi:10.1126/science.1158684

57. Uetz P, Giot L, Cagney G, et al. A comprehensive analysis of protein-protein interactions inSaccharomyces cerevisiae. Nature. 2000;403(6770):623-627. doi:10.1038/35001009

58. Ito T, Chiba T, Ozawa R, Yoshida M, Hattori M, Sakaki Y. A comprehensive twohybrid analysis to explore the yeast protein interactome. Proc Natl Acad Sci U SA. 2001;98(8):4569-4574. doi:10.1073/pnas.061034498

59. Fromont-Racine M, Mayes AE, Brunet-Simon A, et al. Genome-Wide Protein Interaction Screens Reveal Functional Networks Involving Sm-Like Proteins. Yeast. 2000;1(2):95-110. doi:10.1002/1097-0061(20000630)17:2<95::aidyea16>3.0.co;2-h

60. Hirst M, Ho C, Sabourin L, Rudnicki M, Penn L, Sadowski I. A two-hybrid system for transactivator bait proteins. Proc Natl Acad Sci. 2001;98(15):8726-8731. doi:10.1073/PNAS.141413598

61. Urech DM, Lichtlen P, Barberis A. Cell growth selection system to detect extracellular and transmembrane protein interactions. Biochim Biophys Acta - 
Gen Subj. 2003. doi:10.1016/S0304-4165(03)00133-8

62. Mallick J, Jansen G, Wu C, Whiteway M. SRYTH: A new yeast two-hybrid method. In: Methods in Molecular Biology. Vol 1356. ; 2016:31-41. doi:10.1007/978-14939-3052-4_3

63. Babu M, Krogan NJ, Awrey DE, Emili A, Greenblatt JF. Systematic characterization of the protein interaction network and protein complexes in saccharomyces cerevisiae using tandem affinity purification and mass spectrometry. Methods Mol Biol. 2009;548:187-207. doi:10.1007/978-1-59745-540-4_11

64. Ho Y, Gruhler A, Heilbut A, et al. Systematic identification of protein complexes in Saccharomyces cerevisiae by mass spectrometry. Nature. 2002. doi:10.1038/415180a

65. Piovesan D, Giollo M, Ferrari C, Tosatto SCE. Protein function prediction using guilty by association from interaction networks. Amino Acids. 2015;47(12):25832592. doi:10.1007/s00726-015-2049-3

66. Pitre S, Hooshyar M, Schoenrock A, et al. Short Co-occurring Polypeptide Regions Can Predict Global Protein Interaction Maps. Sci Rep. 2012;2:1-10. doi:10.1038/srep00239

67. Ciccia A, Elledge SJ. The DNA damage response: making it safe to play with knives. Mol Cell. 2010;40(2):179-204. doi:10.1016/j.molcel.2010.09.019 
68. Hoeijmakers JHJ. DNA Damage, Aging, and Cancer. N Engl J Med. 2009;361(15):1475-1485. doi:10.1056/NEJMra0804615

69. Lindahl T, Barnes DE. Repair of endogenous DNA damage. Cold Spring Harb Symp Quant Biol. 2000;65:127-133. doi:10.1101/SQB.2000.65.127

70. Saleh-Gohari N, Bryant H, biology NS-... and cellular, 2005 undefined. Spontaneous homologous recombination is induced by collapsed replication forks that are caused by endogenous DNA single-strand breaks. Am Soc Microbiol. https://mcb.asm.org/content/25/16/7158.short. Accessed February 19, 2019.

71. Cadet J, Wagner JR. DNA base damage by reactive oxygen species, oxidizing agents, and UV radiation. Cold Spring Harb Perspect Biol. 2013;5(2):a012559. doi:10.1101/cshperspect.a012559

72. Jiricny J. The multifaceted mismatch-repair system. Nat Rev Mol Cell Biol. 2006;7(5):335-346. doi:10.1038/nrm1907

73. Nakamura J, Walker VE, Upton PB, Chiang SY, Kow YW, Swenberg JA. Highly sensitive apurinic/apyrimidinic site assay can detect spontaneous and chemically induced depurination under physiological conditions. Cancer Res. 1998;58(2):222225. http://www.ncbi.nlm.nih.gov/pubmed/9443396. Accessed September 16, 2019.

74. Lindahl T, Nyberg B. Rate of depurination of native deoxyribonucleic acid. Biochemistry. 1972;11(19):3610-3618. doi:10.1021/bi00769a018 
75. Talpaert-Borlè M. Formation, detection and repair of AP sites. Mutat Res -

Fundam Mol Mech Mutagen. 1987;181(1):45-56. doi:10.1016/0027-

5107(87)90286-7

76. Guillet M, Boiteux S. Endogenous DNA abasic sites cause cell death in the absence of Apn1, Apn2 and Rad1/Rad10 in Saccharomyces cerevisiae. EMBO J.

2002;21(11):2833-2841. doi:10.1093/emboj/21.11.2833

77. Dianov GL, Sleeth KM, Dianova II, Allinson SL. Repair of abasic sites in DNA. Mutat Res Mol Mech Mutagen. 2003;531(1-2):157-163.

doi:10.1016/J.MRFMMM.2003.09.003

78. Alizadeh E, Orlando TM, Sanche L. Biomolecular Damage Induced by lonizing Radiation: The Direct and Indirect Effects of Low-Energy Electrons on DNA. Annu Rev Phys Chem. 2015;66(1):379-398. doi:10.1146/annurev-physchem-040513103605

79. Rastogi RP, Richa, Kumar A, Tyagi MB, Sinha RP. Molecular mechanisms of ultraviolet radiation-induced DNA damage and repair. J Nucleic Acids. 2010;2010:592980. doi:10.4061/2010/592980

80. Yokoyama H, Mizutani R. Structural biology of DNA (6-4) photoproducts formed by ultraviolet radiation and interactions with their binding proteins. Int J Mol Sci. 2014;15(11):20321-20338. doi:10.3390/ijms151120321

81. Weng $\mathrm{M}$, Zheng $\mathrm{Y}$, Jasti $\mathrm{VP}$, et al. Repair of mitomycin $\mathrm{C}$ mono- and interstrand 
cross-linked DNA adducts by UvrABC: a new model. Nucleic Acids Res. 2010;38(20):6976-6984. doi:10.1093/nar/gkq576

82. Sugiyama M, Kumagai T. Molecular and structural biology of bleomycin and its resistance determinants. J Biosci Bioeng. 2002;93(2):105-116. doi:10.1016/S13891723(02)80001-9

83. Koç A, Wheeler LJ, Mathews CK, Merrill GF. Hydroxyurea Arrests DNA Replication by a Mechanism that Preserves Basal dNTP Pools. J Biol Chem. 2004;279(1):223230. doi:10.1074/jbc.M303952200

84. Malkova A, Haber JE. Mutations Arising During Repair of Chromosome Breaks. Annu Rev Genet. 2012;46(1):455-473. doi:10.1146/annurev-genet-110711155547

85. Lieber MR. The mechanism of human nonhomologous DNA End joining. J Biol Chem. 2008. doi:10.1074/jbc.R700039200

86. Deriano L, Roth DB. Modernizing the Nonhomologous End-Joining Repertoire: Alternative and Classical NHEJ Share the Stage. Annu Rev Genet. 2013. doi:10.1146/annurev-genet-110711-155540

87. Aggarwal M, Brosh RM. Functional analyses of human DNA repair proteins important for aging and genomic stability using yeast genetics. DNA Repair (Amst). 2012. doi:10.1016/j.dnarep.2012.01.013 
88. Jiang B, Glover JNM, Weinfeld M. Neurological disorders associated with DNA strand-break processing enzymes. Mech Ageing Dev. 2017;161(Pt A):130-140. doi:10.1016/j.mad.2016.07.009

89. Recio MJ, Dominguez-Pinilla N, Perrig MS, et al. Extreme phenotypes with identical mutations: Two patients with same non-sense NHEJ1 homozygous mutation. Front Immunol. 2019;10(JAN). doi:10.3389/fimmu.2018.02959

90. Chiruvella KK, Liang Z, Wilson TE. Repair of double-strand breaks by end joining. Cold Spring Harb Perspect Biol. 2013. doi:10.1101/cshperspect.a012757

91. Kowalczykowski SC. An Overview of the Molecular Mechanisms of Recombinational DNA Repair. Cold Spring Harb Perspect Biol. 2015;7(11). doi:10.1101/cshperspect.a016410

92. Lieber MR. The Mechanism of Double-Strand DNA Break Repair by the Nonhomologous DNA End-Joining Pathway. Annu Rev Biochem. 2010;79(1):181211. doi:10.1146/annurev.biochem.052308.093131

93. Liang B, Qiu J, Ratnakumar K, Laurent BC. RSC Functions as an Early DoubleStrand-Break Sensor in the Cell's Response to DNA Damage. Curr Biol. 2007;17(16):1432-1437. doi:10.1016/j.cub.2007.07.035

94. Bradbury JM, Jackson SP. The complex matter of DNA double-strand break detection. Biochem Soc Trans. 2003;31(1):40-44. doi:10.1042/bst0310040 
95. Zou L, Elledge SJ. Sensing DNA damage through ATRIP recognition of RPA-ssDNA complexes. Science (80- ). 2003. doi:10.1126/science.1083430

96. Bandhu A, Kang J, Fukunaga K, Goto G, Sugimoto K. Ddc2 Mediates Mec1 Activation through a Ddc1- or Dpb11-Independent Mechanism. Toczyski DP, ed. PLoS Genet. 2014;10(2):e1004136. doi:10.1371/journal.pgen.1004136

97. Iwasaki D, Hayashihara K, Shima H, et al. The MRX Complex Ensures NHEJ Fidelity through Multiple Pathways Including Xrs2-FHA-Dependent Tel1 Activation. JinksRobertson S, ed. PLOS Genet. 2016;12(3):e1005942.

doi:10.1371/journal.pgen.1005942

98. Van Attikum H, Fritsch O, Gasser SM. Distinct roles for SWR1 and INO80 chromatin remodeling complexes at chromosomal double-strand breaks. EMBO J. 2007;26(18):4113-4125. doi:10.1038/sj.emboj.7601835

99. Downs JA, Allard S, Jobin-Robitaille O, et al. Binding of chromatin-modifying activities to phosphorylated histone $\mathrm{H} 2 \mathrm{~A}$ at DNA damage sites. Mol Cell. 2004;16(6):979-990. doi:10.1016/j.molcel.2004.12.003

100. Price BD, D'Andrea AD. Chromatin remodeling at DNA double-strand breaks. Cell. 2013;152(6):1344-1354. doi:10.1016/j.cell.2013.02.011

101. Morrison AJ, Shen X. Chromatin remodelling beyond transcription: The INO80 and SWR1 complexes. Nat Rev Mol Cell Biol. 2009;10(6):373-384. doi:10.1038/nrm2693 
102. Horigome C, Oma Y, Konishi T, et al. SWR1 and INO80 chromatin remodelers contribute to dna double-strand break perinuclear anchorage site choice. $\mathrm{Mol}$ Cell. 2014;55(4):626-639. doi:10.1016/j.molcel.2014.06.027

103. Xu Y, Ayrapetov MK, Xu C, Gursoy-Yuzugullu O, Hu Y, Price BD. Histone H2A.Z Controls a Critical Chromatin Remodeling Step Required for DNA Double-Strand Break Repair. Mol Cell. 2012;48(5):723-733. doi:10.1016/j.molcel.2012.09.026

104. Chambers AL, Brownlee PM, Durley SC, Beacham T, Kent NA, Downs JA. The two different isoforms of the RSC chromatin remodeling complex play distinct roles in DNA damage responses. PLoS One. 2012;7(2). doi:10.1371/journal.pone.0032016

105. Lazzaro F, Sapountzi V, Granata M, et al. Histone methyltransferase Dot1 and Rad9 inhibit single-stranded DNA accumulation at DSBs and uncapped telomeres. EMBO J. 2008. doi:10.1038/emboj.2008.81

106. Chapman JR, Taylor MRG, Boulton SJ. Playing the End Game: DNA Double-Strand Break Repair Pathway Choice. Mol Cell. 2012;47(4):497-510. doi:10.1016/j.molcel.2012.07.029

107. Shrivastav M, De Haro LP, Nickoloff JA. Regulation of DNA double-strand break repair pathway choice. Cell Res. 2008;18(1):134-147. doi:10.1038/cr.2007.111

108. Symington LS, Gautier J. Double-Strand Break End Resection and Repair Pathway Choice. Annu Rev Genet. 2011. doi:10.1146/annurev-genet-110410-132435 
109. Cannavo E, Johnson D, Andres SN, et al. Regulatory control of DNA end resection by Sae2 phosphorylation. Nat Commun. 2018. doi:10.1038/s41467-018-06417-5

110. Chen X, Niu H, Chung WH, et al. Cell cycle regulation of DNA double-strand break end resection by Cdk1-dependent Dna2 phosphorylation. Nat Struct Mol Biol. 2011. doi:10.1038/nsmb.2105

111. Chen X, Niu H, Yu Y, et al. Enrichment of Cdk1-cyclins at DNA double-strand breaks stimulates Fun30 phosphorylation and DNA end resection. Nucleic Acids Res. 2016;44(6):2742-2753. doi:10.1093/nar/gkv1544

112. Chen H, Donnianni RA, Handa N, et al. Sae2 promotes DNA damage resistance by removing the Mre11-Rad50-Xrs2 complex from DNA and attenuating Rad53 signaling. Proc Natl Acad Sci U S A. 2015;112(15):E1880-E1887. doi:10.1073/pnas.1503331112

113. Godin SK, Sullivan MR, Bernstein KA. Novel insights into RAD51 activity and regulation during homologous recombination and DNA replication. Biochem Cell Biol. 2016. doi:10.1139/bcb-2016-0012

114. Krogh BO, Symington LS. Recombination Proteins in Yeast. Annu Rev Genet. 2004;38(1):233-271. doi:10.1146/annurev.genet.38.072902.091500

115. Jasin M, Rothstein R. Repair of strand breaks by homologous recombination. Cold Spring Harb Perspect Biol. 2013;5(11):a012740. doi:10.1101/cshperspect.a012740 
116. Krasner DS, Daley JM, Sung P, Niu H. Interplay between Ku and replication protein A in the restriction of Exo1-mediated DNA break end resection. J Biol Chem. 2015. doi:10.1074/jbc.M115.660191

117. Wu D, Topper LM, Wilson TE. Recruitment and dissociation of nonhomologous end joining proteins at a DNA double-strand break in Saccharomyces cerevisiae. Genetics. 2008;178(3):1237-1249. doi:10.1534/genetics.107.083535

118. Emerson $\mathrm{CH}$, Bertuch AA. Consider the workhorse: Nonhomologous end-joining in budding yeast ${ }^{1}$. Biochem Cell Biol. 2016;94(5). doi:10.1139/bcb-2016-0001

119. Zhang Y, Hefferin ML, Chen L, et al. Role of Dnl4-Lif1 in nonhomologous endjoining repair complex assembly and suppression of homologous recombination. Nat Struct Mol Biol. 2007. doi:10.1038/nsmb1261

120. Hopfner KP, Karcher A, Craig L, Woo TT, Carney JP, Tainer JA. Structural biochemistry and interaction architecture of the DNA double-strand break repair Mre11 nuclease and Rad50-ATPase. Cell. 2001. doi:10.1016/S00928674(01)00335-X

121. Chen L, Trujillo K, Ramos W, Sung P, Tomkinson AE. Promotion of Dnl4-Catalyzed DNA end-joining by the Rad50/Mre11/Xrs2 and Hdf1/Hdf2 complexes. Mol Cell. 2001. doi:10.1016/S1097-2765(01)00388-4

122. Valencia M, Bentele M, Vaze MB, et al. NEJ1 controls non-homologous end joining in Saccharomyces cerevisiae. Nature. 2001. doi:10.1038/414666a 
123. Chen X, Tomkinson AE. Yeast Nej1 is a key participant in the initial end binding and final ligation steps of nonhomologous end joining. J Biol Chem.

2011;286(6):4931-4940. doi:10.1074/jbc.M110.195024

124. Bahmed K, Nitiss KC, Nitiss JL. Yeast Tdp1 regulates the fidelity of nonhomologous end joining. Proc Natl Acad Sci. 2010;107(9):4057-4062.

doi:10.1073/pnas.0909917107

125. Tseng HM, Tomkinson AE. Processing and joining of DNA ends coordinated by interactions among Dnl4/Lif1, Pol4, and FEN-1. J Biol Chem. 2004;279(46):4758047588. doi:10.1074/jbc.M404492200

126. Tseng SF, Gabriel A, Teng SC. Proofreading activity of DNA polymerase Pol2 mediates 3 '-end processing during nonhomologous end joining in yeast. PLoS Genet. 2008. doi:10.1371/journal.pgen.1000060

127. Yang H, Matsumoto Y, Trujillo KM, Lees-Miller SP, Osley MA, Tomkinson AE. Role of the yeast DNA repair protein Nej1 in end processing during the repair of DNA double strand breaks by non-homologous end joining. DNA Repair (Amst). 2015. doi:10.1016/j.dnarep.2015.04.003

128. Bebenek K, Garcia-Biaz M, Patishall SM, Kunkel TA. Biochemical properties of Saccharomyces cerevisiae DNA polymerase IV. J Biol Chem. 2005. doi:10.1074/jbc.M501981200

129. Ruiz JF, Pardo B, Sastre-Moreno G, Aguilera A, Blanco L. Yeast Pol4 Promotes 
Tel1-Regulated Chromosomal Translocations. PLoS Genet. 2013.

doi:10.1371/journal.pgen.1003656

130. Mahaney BL, Lees-Miller SP, Cobb JA. The C-terminus of Nej1 is critical for nuclear localization and non-homologous end-joining. DNA Repair (Amst). 2014. doi:10.1016/j.dnarep.2013.12.002

131. Jessulat M, Malty RH, Nguyen-Tran D-H, et al. Spindle Checkpoint Factors Bub1 and Bub2 Promote DNA Double-Strand Break Repair by Nonhomologous End Joining. Mol Cell Biol. 2015;35(14):2448-2463. doi:10.1128/MCB.00007-15

132. Yu L, Volkert MR. Differential Requirement for SUB1 in Chromosomal and Plasmid Double-Strand DNA Break Repair. PLoS One. 2013;8(3). doi:10.1371/journal.pone.0058015

133. Yu L, Ma H, Ji X, Volkert MR. The Sub1 nuclear protein protects DNA from oxidative damage. Mol Cell Biochem. 2016;412(1-2):165-171. doi:10.1007/s11010-015-2621-x

134. Kang S g., Saven JG. Computational protein design: structure, function and combinatorial diversity. Curr Opin Chem Biol. 2007;11(3):329-334. doi:10.1016/j.cbpa.2007.05.006

135. Pantazes R, Grisewood M, Maranas C. Recent advances in computational protein design. Curr Opin Struct Biol. 2011;21(4):467-472. doi:doi: 10.1016/j.sbi.2011.04.005 
136. Saven JG. Computational protein design: Advances in the design and redesign of biomolecular nanostructures. Curr Opin Colloid Interface Sci. 2010;15(1-2):13-17. doi:10.1016/j.cocis.2009.06.002

137. Yu K, Liu C, Kim B-G, Lee D-Y. Synthetic fusion protein design and applications. Biotechnol Adv. 2014;33(1):155-164. doi:10.1016/j.biotechadv.2014.11.005

138. Colombo M, Mizzotti C, Masiero S, Kater MM, Pesaresi P. Peptide aptamers: The versatile role of specific protein function inhibitors in plant biotechnology. J Integr Plant Biol. 2015;57(11):892-901. doi:10.1111/jipb.12368

139. Goldflam M, Ullman CG. Recent Advances Toward the Discovery of Drug-Like Peptides De novo. Front Chem. 2015;3:69. doi:10.3389/fchem.2015.00069

140. Benjamin Stranges $P$, Kuhlman B. A comparison of successful and failed protein interface designs highlights the challenges of designing buried hydrogen bonds. Protein Sci. 2013;22(1):74-82. doi:10.1002/pro.2187

141. Karanicolas J, Kuhlman B. Computational design of affinity and specificity at protein-protein interfaces. Curr Opin Struct Biol. 2009;19(4):458-463. doi:10.1016/j.sbi.2009.07.005

142. Mikut R, Ruden S, Reischl M, Breitling F, Volkmer R, Hilpert K. Improving short antimicrobial peptides despite elusive rules for activity. Biochim Biophys Acta Biomembr. 2016;1858(5):1024-1033. doi:10.1016/j.bbamem.2015.12.013 
143. Schreiber G, Fleishman SJ. Computational design of protein-protein interactions. Curr Opin Struct Biol. 2013;23(6):903-910. doi:10.1016/j.sbi.2013.08.003

144. Chica RA, Doucet N, Pelletier JN. Semi-rational approaches to engineering enzyme activity: Combining the benefits of directed evolution and rational design. Curr Opin Biotechnol. 2005;16(4):378-384. doi:10.1016/j.copbio.2005.06.004

145. Woolfson DN, Bartlett GJ, Burton AJ, et al. De novo protein design: How do we expand into the universe of possible protein structures? Curr Opin Struct Biol. 2015;33:16-26. doi:10.1016/j.sbi.2015.05.009

146. Cherkasov A, Hilpert $\mathrm{K}$, Jenssen $\mathrm{H}$, et al. Use of Artificial Intelligence in the Design of Small Peptide Antibiotics Effective against a Broad Spectrum of Highly Antibiotic-Resistant Superbugs. ACS Chem Biol. 2009;4(1):65-74. doi:10.1021/cb800240j

147. Devlin JJ, Panganiban LC, Devlin PE. Random peptide libraries: A source of specific protein binding molecules. Science (80- ). 1990;249(4967):404-406. doi:10.1126/science.2143033

148. Li Z, Yang Y, Zhan J, Dai L, Zhou Y. Energy Functions in De Novo Protein Design: Current Challenges and Future Prospects. Annu Rev Biophys. 2013;42(1):315-335. doi:10.1146/annurev-biophys-083012-130315

149. Luisi PL, Chiarabelli C, Stano P. From never born proteins to minimal living cells: Two projects in synthetic biology. Orig Life Evol Biosph. 2006;36(5-6):605-616. 
doi:10.1007/s11084-006-9033-6

150. Craik DJ, Fairlie DP, Liras S, Price D. The Future of Peptide-based Drugs. Chem Biol Drug Des. 2013;81(1):136-147. doi:10.1111/cbdd.12055

151. Otvos L, Wade JD. Current challenges in peptide-based drug discovery. Front Chem. 2014;2(62):1-4. doi:10.3389/fchem.2014.00062

152. Fosgerau K, Hoffmann T. Peptide therapeutics: Current status and future directions. Drug Discov Today. 2015;20(1):122-128. doi:10.1016/j.drudis.2014.10.003

153. Kaspar AA, Reichert JM. Future directions for peptide therapeutics development. Drug Discov Today. 2013;18(17-18):807-817. doi:10.1016/j.drudis.2013.05.011

154. Tinberg CE, Khare SD, Dou J, et al. Computational design of ligand-binding proteins with high affinity and selectivity. Nature. 2013;501(7466):212-216. doi:10.1038/nature12443

155. Wilson CJ. Rational protein design: Developing next-generation biological therapeutics and nanobiotechnological tools. Wiley Interdiscip Rev Nanomedicine Nanobiotechnology. 2015;7(3):330-341. doi:10.1002/wnan.1310

156. Lao BB, Drew K, Guarracino DA, et al. Rational design of topographical helix mimics as potent inhibitors of protein-protein interactions. J Am Chem Soc. 2014;136(22):7877-7888. doi:10.1021/ja502310r 
157. Huang $\mathrm{Y}$, Jiang $\mathrm{Y}$, Wang $\mathrm{H}$, et al. Curb challenges of the "Trojan Horse" approach: Smart strategies in achieving effective yet safe cell-penetrating peptide-based drug delivery. Adv Drug Deliv Rev. 2013;65(10):1299-1315. doi:10.1016/j.addr.2012.11.007

158. Takeuchi T, Popiel HA, Futaki S, Wada K, Nagai Y. Peptide-based therapeutic approaches for treatment of the polyglutamine diseases. Curr Med Chem. 2014;21(23):2575-2582. doi:10.2174/0929867321666140217124038

159. Chevalier A, Silva D-A, Rocklin GJ, et al. Massively parallel de novo protein design for targeted therapeutics. Nature. 2017;550(7674):74. doi:10.1038/nature23912

160. Viart B, Dias-Lopes C, Kozlova E, et al. EPI-peptide designer: A tool for designing peptide ligand libraries based on epitope-paratope interactions. Bioinformatics. 2016;32(10):1462-1470. doi:10.1093/bioinformatics/btw014

161. Ostrem JML, Shokat KM. Direct small-molecule inhibitors of KRAS: from structural insights to mechanism-based design. Nat Rev Drug Discov. 2016;15(11):771-785. doi:10.1038/nrd.2016.139

162. S.M. L, B.A. K. Anchored design of protein-protein interfaces. PLoS One. $2011 ; 6(6)$.

163. Pierce BG, Wiehe K, Hwang H, Kim BH, Vreven T, Weng Z. ZDOCK server: Interactive docking prediction of protein-protein complexes and symmetric multimers. Bioinformatics. 2014;30(12):1771-1773. 
doi:10.1093/bioinformatics/btu097

164. Fisher MA, Mckinley KL, Bradley LH, Viola SR, Hecht MH. De Novo Designed Proteins from a Library of Artificial Sequences Function in Escherichia Coli and Enable Cell Growth. PLoS One. 2011;6(1):15364.

doi:10.1371/journal.pone.0015364

165. Grigoryan G, Reinke AW, Keating AE. Design of protein-interaction specificity gives selective bZIP-binding peptides. Nature. 2009;458(7240):859-864. doi:10.1038/nature07885

166. Negron C, Keating AE. Multistate protein design using CLEVER and CLASSY. In: Methods in Enzymology. Vol 523. ; 2013:171-190. doi:10.1016/B978-0-12$394292-0.00008-4$

167. Davey JA, Chica RA. Multistate approaches in computational protein design. Protein Sci. 2012;21(9):1241-1252. doi:10.1002/pro.2128

168. Neduva V, Russell RB. Peptides mediating interaction networks: new leads at last. Curr Opin Biotechnol. 2006;17(5):465-471. doi:10.1016/j.copbio.2006.08.002

169. Pitre S, North C, Alamgir M, et al. Global investigation of protein-protein interactions in yeast Saccharomyces cerevisiae using re-occurring short polypeptide sequences. Nucleic Acids Res. 2008;36(13):4286-4294. doi:10.1093/nar/gkn390 
170. Yungki P. Critical assessment of sequence-based protein-protein interaction prediction methods that do not require homologous protein sequences. $B M C$ Bioinformatics. 2009;10:419. doi:10.1186/1471-2105-10-419

171. Kohalmi S, Nowak J, Crosby W. The Yeast Two-Hybrid System. (Hansen E, Harper G, eds.). London: Taylor and Francis Ltd.; 2002.

172. Gelperin DM, White MA, Wilkinson ML, et al. Biochemical and genetic analysis of the yeast proteome with a movable ORF collection. Genes Dev. 2005;19(23):28162826. doi:10.1101/gad.1362105

173. Clonetech Laboratories Inc. Clonetech - Yeast Protocols Handbook.; 2009. www.clonetech.com.

174. Jia CYH, Nie J, Wu C, Li C, Li SS-C. Novel Src Homology 3 Domain-binding Motifs Identified from Proteomic Screen of a Pro-rich Region. Mol Cell Proteomics. 2005;4(8):1155-1166. doi:10.1074/mcp.M500108-MCP200

175. Schoenrock A, Burnside D, Moteshareie $H$, et al. Engineering inhibitory proteins with InSiPS: The In-Silico Protein Synthesizer. Proc Int Conf High Perform Comput Networking, Storage Anal - SC '15. 2015:1-11.

http://dl.acm.org/citation.cfm?doid=2807591.2807630.

176. Hanway D, Chin JK, Xia G, Oshiro G, Winzeler EA, Romesberg FE. Previously uncharacterized genes in the UV- and MMS-induced DNA damage response in yeast. Proc Natl Acad Sci U S A. 2002;99(16):10605-10610. 
doi:10.1073/pnas.152264899

177. Brown JA, Sherlock G, Myers CL, et al. Global analysis of gene function in yeast by quantitative phenotypic profiling. Mol Syst Biol. 2006;2:2006 0001. doi:10.1038/msb4100043

178. Zhang Q, Petrey D, Deng L, Qiang L, Shi Y, Thu C. Structure-based prediction of protein-protein interactions on a genome-wide scale. Nature. 2013;495(7421):556-560. http://www.nature.com/nature/journal/v490/n7421/abs/nature11503.html. Accessed April 18, 2017.

179. Huang P-S, Boyken SE, Baker D. The coming of age of de novo protein design. Nature. 2016;537(7620):320-327. doi:10.1038/nature19946

180. Thomson AR, Wood CW, Burton AJ, et al. Computational design of watersoluble -helical barrels. Science (80- ). 2014;346(6208):485-488. doi:10.1126/science.1257452

181. Huang P-S, Oberdorfer G, Xu C, et al. High thermodynamic stability of parametrically designed helical bundles. Science (80- ). 2014;346(6208):481-485. doi:10.1126/science.1257481

182. King NP, Sheffler W, Sawaya MR, et al. Computational Design of Self-Assembling Protein Nanomaterials with Atomic Level Accuracy. Science (80- ). 2012;336(6085):1171-1174. doi:10.1126/science.1219364 
183. Boyken SE, Chen Z, Groves B, et al. De novo design of protein homo-oligomers with modular hydrogen-bond network-mediated specificity. Science (80- ). 2016;352(6286):680-687. doi:10.1126/science.aad8865

184. Hanley-Bowdoin L, Lopez-Ochoa L. Peptide aptamers that bind to the rep proteins of ssDNA viruses. US Pat 9,102,705. 2015.

https://www.google.com/patents/US9102705. Accessed April 18, 2017.

185. Rafferty J, Nagaraj H, P. McCloskey A, et al. Peptide Therapeutics and the Pharmaceutical Industry: Barriers Encountered Translating from the Laboratory to Patients. Curr Med Chem. 2016;23(37):4231-4259. doi:10.2174/0929867323666160909155222

186. Biyani M, Kawai K, Kitamura K, et al. PEP-on-DEP: A competitive peptide-based disposable electrochemical aptasensor for renin diagnostics. Biosens Bioelectron. 2016;84:120-125. doi:10.1016/j.bios.2015.12.078

187. Yu X, Yang Y-P, Dikici E, Deo SK, Daunert S. Beyond Antibodies as Binding Partners: The Role of Antibody Mimetics in Bioanalysis. Annu Rev Anal Chem. 2017;10(1):293-320. doi:10.1146/annurev-anchem-061516-045205

188. Mehta A, Haber JE. Sources of DNA double-strand breaks and models of recombinational DNA repair. Cold Spring Harb Perspect Biol. 2014;6(9):a016428. doi:10.1101/cshperspect.a016428

189. Clikeman JA, Khalsa J, Barton SL, Nickoloff JA. Homologous recombinational repair 
of double-strand breaks in yeast is enhanced by MAT heterozygosity through yKU-dependent and -independent mechanisms. Genetics. 2001;157(2):579-589.

190. Zhu Z, Chung W-H, Shim EY, Lee SE, Ira G. Sgs1 Helicase and Two Nucleases Dna2 and Exo1 Resect DNA Double-Strand Break Ends. Cell. 2008;134(6):981-994. doi:10.1016/J.CELL.2008.08.037

191. Richard P, Ogami K, Chen Y, et al. NRDE-2, the human homolog of fission yeast Nrl1, prevents DNA damage accumulation in human cells. RNA Biol. 2018;15(7):868-876. doi:10.1080/15476286.2018.1467180

192. Gao S, Honey S, Futcher B, Grollman AP. The non-homologous end-joining pathway of S. cerevisiae works effectively in G1-phase cells, and religates cognate ends correctly and non-randomly. DNA Repair (Amst). 2016;42:1-10. doi:10.1016/j.dnarep.2016.03.013

193. Jain S, Sugawara N, Mehta A, Ryu T, Haber JE. Sgs1 and Mph1 helicases enforce the recombination execution checkpoint during DNA double-strand break repair in saccharomyces cerevisiae. Genetics. 2016. doi:10.1534/genetics.115.184317

194. Krejci L, Altmannova V, Spirek M, Zhao X. Homologous recombination and its regulation. Nucleic Acids Res. 2012;40(13):5795-5818. doi:10.1093/nar/gks270

195. Wu X, Wang Z. Relationships between yeast Rad27 and Apn1 in response to apurinic/apyrimidinic (AP) sites in DNA. Nucleic Acids Res. 1999. doi:10.1093/nar/27.4.956 
196. Daley JM, Niu H, Miller AS, Sung P. Biochemical mechanism of DSB end resection and its regulation. DNA Repair (Amst). 2015. doi:10.1016/j.dnarep.2015.04.015

197. Mantiero D, Clerici M, Lucchini G, Longhese MP. Dual role for Saccharomyces cerevisiae Tel1 in the checkpoint response to double-strand breaks. EMBO Rep. 2007;8(4):380-387. doi:10.1038/sj.embor.7400911

198. Rutter J. Essay: Amersham Biosciences and Science Prize. PAS domains and metabolic status signaling. Science. 2002;298(5598):1567-1568. doi:10.1126/science. 1080001

199. Crespo JL, Hall MN. Elucidating TOR Signaling and Rapamycin Action: Lessons from Saccharomyces cerevisiae. Microbiol Mol Biol Rev. 2003. doi:10.1128/mmbr.66.4.579-591.2002

200. Grose JH, Smith TL, Sabic H, Rutter J. Yeast PAS kinase coordinates glucose partitioning in response to metabolic and cell integrity signaling. EMBO J. 2007. doi:10.1038/sj.emboj.7601914

201. Smith TL, Rutter J. Regulation of glucose partitioning by PAS kinase and Ugp1 phosphorylation. Mol Cell. 2007;26(4):491-499. doi:10.1016/j.molcel.2007.03.025

202. Huang M, Xu Q, Mitsui K, Xu Z. PSK1 regulates expression of SOD1 involved in oxidative stress tolerance in yeast. FEMS Microbiol Lett. 2014;350(2):154-160. doi:10.1111/1574-6968.12329 
203. Rutter J, Probst BL, McKnight SL. Coordinate regulation of sugar flux and translation by PAS kinase. Cell. 2002;111(1):17-28. doi:10.1016/S00928674(02)00974-1

204. Rowe LA, Degtyareva N, Doetsch PW. DNA Damage-induced Reactive Oxygen Species (ROS) Stress Response in Saccharomyces cerevisiae. Free Radic Biol Med. 2008;45(8):1167. doi:10.1016/J.FREERADBIOMED.2008.07.018

205. Winzeler EA, Shoemaker DD, Astromoff A, et al. Functional characterization of the S. cerevisiae genome by gene deletion and parallel analysis. Science. 1999;285(5429):901-906. doi:10.1126/science.285.5429.901

206. Sopko R, Huang D, Preston N, et al. Mapping Pathways and Phenotypes by Systematic Gene Overexpression. Mol Cell. 2006;21(3):319-330. doi:10.1016/J.MOLCEL.2005.12.011

207. Omidi K, Hooshyar M, Jessulat M, et al. Phosphatase complex Pph3/Psy2 is involved in regulation of efficient non-homologous end-joining pathway in the yeast Saccharomyces cerevisiae. PLoS One. 2014;9(1). doi:10.1371/journal.pone.0087248

208. Jessulat M, Alamgir M, Salsali H, Greenblatt J, Xu J, Golshani A. Interacting proteins Rtt109 and Vps75 affect the efficiency of non-homologous end-joining in Saccharomyces cerevisiae. Arch Biochem Biophys. 2008;469(2):157-164. doi:10.1016/j.abb.2007.11.001 
209. Tong AHY, Boone C. Synthetic genetic array analysis in Saccharomyces cerevisiae. Methods Mol Biol. 2006;313(1):171-192. doi:10.1016/S0076-6879(10)70007-0

210. Kuzmin E, Sharifpoor, Sara; Baryshnikova, Anastasia; Costanzo M, Myers CL., AndrewsJ.; B, Boone C. Synthetic Genetic Array Analysis for Global Mapping of Genetic Networks in Yeast. Yeast Genet Methods Protoc. 2014;1205:303-328. doi:10.1007/978-1-4939-1363-3

211. Ogiwara H, Ui A, Enomoto T, Seki M. Role of Elg1 protein in double strand break repair. Nucleic Acids Res. 2007;35(2):353-362. doi:10.1093/nar/gkl1027

212. Eide L, Bjørås M, Pirovano M, Alseth I, Berdal KG, Seeberg E. Base excision of oxidative purine and pyrimidine DNA damage in Saccharomyces cerevisiae by a DNA glycosylase with sequence similarity to endonuclease III from Escherichia coli. Proc Natl Acad Sci U S A. 1996;93(20):10735-10740. doi:10.1073/pnas.93.20.10735

213. van Leeuwen J, Pons C, Boone C, Andrews BJ. Mechanisms of suppression: The wiring of genetic resilience. Bioessays. 2017;39(7). doi:10.1002/bies.201700042

214. Driscoll R, Hudson A, Jackson SP. Yeast Rtt109 promotes genome stability by acetylating histone H3 on lysine 56. Science. 2007;315(5812):649-652. doi:10.1126/science.1135862

215. Baryshnikova A, Costanzo M, Kim Y, et al. Quantitative analysis of fitness and genetic interactions in yeast on a genome scale. Nat Methods. 2010;7(12):1017- 
1024. doi:10.1038/nmeth.1534

216. Koh JLY, Ding H, Costanzo M, et al. DRYGIN: A database of quantitative genetic interaction networks in yeast. Nucleic Acids Res. 2009;38(SUPPL.1). doi:10.1093/nar/gkp820

217. Boone $\mathrm{C}$, Bussey $\mathrm{H}$, Andrews BJ. Exploring genetic interactions and networks with yeast. Nat Rev Genet. 2007;8(6):437-449. doi:10.1038/nrg2085

218. Duch A, Canal B, Barroso SI, et al. Multiple signaling kinases target Mrc1 to prevent genomic instability triggered by transcription-replication conflicts. Nat Commun. 2018;9(1). doi:10.1038/s41467-017-02756-x

219. Tsolou A, Lydall D. Mrc1 protects uncapped budding yeast telomeres from exonuclease EXO1. DNA Repair (Amst). 2007;6(11):1607-1617. doi:10.1016/j.dnarep.2007.05.010

220. Schipler A, Iliakis G. DNA double-strand-break complexity levels and their possible contributions to the probability for error-prone processing and repair pathway choice. Nucleic Acids Res. 2013;41(16):7589-7605. doi:10.1093/nar/gkt556

221. Aparicio T, Baer R, Gautier J. DNA double-strand break repair pathway choice and cancer. DNA Repair (Amst). 2014;19:169-175. doi:10.1016/j.dnarep.2014.03.014

222. Chang HHY, Pannunzio NR, Adachi N, Lieber MR. Non-homologous DNA end joining and alternative pathways to double-strand break repair. Nat Rev Mol Cell 
Biol. 2017;18(8):495-506. doi:10.1038/nrm.2017.48

223. Hefferin ML, Tomkinson AE. Mechanism of DNA double-strand break repair by non-homologous end joining. DNA Repair (Amst). 2005;4(6):639-648. doi:10.1016/j.dnarep.2004.12.005

224. Davis AJ, Chen DJ. DNA double strand break repair via non-homologous endjoining. Trans/ Cancer Res. 2013;2(3):130-143. doi:10.3978/j.issn.2218676X.2013.04.02

225. Sinha M, Peterson CL. Chromatin dynamics during repair of chromosomal DNA double-strand breaks. Epigenomics. 2009;1(2):371-385. doi:10.2217/epi.09.22

226. Shim EY, Hong SJ, Oum J-H, Yanez Y, Zhang Y, Lee SE. RSC Mobilizes Nucleosomes To Improve Accessibility of Repair Machinery to the Damaged Chromatin. Mol Cell Biol. 2007;27(5):1602-1613. doi:10.1128/MCB.01956-06

227. Zhang $\mathrm{Y}$, Smith $\mathrm{CL}$, Saha A, et al. DNA translocation and loop formation mechanism of chromatin remodeling by SWI/SNF and RSC. Mol Cell. 2006;24(4):559-568. doi:10.1016/j.molcel.2006.10.025

228. Szerlong H, Saha A, Cairns BR. The nuclear actin-related proteins Arp7 and Arp9: A dimeric module that cooperates with architectural proteins for chromatin remodeling. EMBO J. 2003;22(12):3175-3187. doi:10.1093/emboj/cdg296

229. Tosi A, Haas C, Herzog F, et al. XStructure and subunit topology of the INO80 
chromatin remodeler and its nucleosome complex. Cell. 2013;154(6).

doi:10.1016/j.cell.2013.08.016

230. Yoshida T, Shimada K, Oma Y, et al. Actin-Related Protein Arp6 Influences H2A.ZDependent and -Independent Gene Expression and Links Ribosomal Protein Genes to Nuclear Pores. Bickmore WA, ed. PLoS Genet. 2010;6(4):e1000910. doi:10.1371/journal.pgen.1000910

231. Wu WH, Alami S, Luk E, et al. Swc2 is a widely conserved H2AZ-binding module essential for ATP-dependent histone exchange. Nat Struct Mol Biol. 2005;12(12):1064-1071. doi:10.1038/nsmb1023

232. Erdemir T, Bilican B, Cagatay T, Goding CR, Yavuzer U. Saccharomyces cerevisiae C1D is implicated in both non-homologous DNA end joining and homologous recombination. Mol Microbiol. 2002;46(4):947-957. doi:10.1046/j.13652958.2002.03224.x

233. Omidi K, Jessulat M, Hooshyar M, et al. Uncharacterized ORF HUR1 influences the efficiency of non-homologous end-joining repair in Saccharomyces cerevisiae. Gene. 2018;639:128-136. doi:10.1016/j.gene.2017.10.003

234. Boulton SJ, Jackson SP. Saccharomyces cerevisiae Ku70 potentiates illegitimate DNA double-strand break repair and serves as a barrier to error-prone DNA repair pathways. EMBO J. 1996;15(18):5093-5103. doi:10.1002/j.14602075.1996.tb00890.x 
235. Higgins NP, Vologodskii A V. Topological Behavior of Plasmid DNA. Microbiol Spectr. 2015;3(2). doi:10.1128/microbiolspec.PLAS-0036-2014

236. Lee SE, Moore JK, Holmes A, Umezu K, Kolodner RD, Haber JE. Saccharomyces Ku70, Mre11/Rad50, and RPA proteins regulate adaptation to G2/M arrest after DNA damage. Cell. 1998;94(3):399-409. doi:10.1016/\$0092-8674(00)81482-8

237. Cagney G, Alvaro D, Reid RJD, Thorpe PH, Rothstein R, Krogan NJ. Functional genomics of the yeast DNA-damage response. Genome Biol. 2006;7(9). doi:10.1186/gb-2006-7-9-233

238. Chiruvella KK, Renard BM, Birkeland SR, Sunder S, Liang Z, Wilson TE. Yeast DNA ligase IV mutations reveal a nonhomologous end joining function of BRCT1 distinct from XRCC4/Lif1 binding. DNA Repair (Amst). 2014;24:37-45. doi:10.1016/j.dnarep.2014.10.003

239. Bétermier M, Bertrand P, Lopez BS. Is Non-Homologous End-Joining Really an Inherently Error-Prone Process? PLoS Genet. 2014;10(1). doi:10.1371/journal.pgen.1004086

240. Alamgir M, Erukova V, Jessulat M, Azizi A, Golshani A. Chemical-genetic profile analysis of five inhibitory compounds in yeast. BMC Chem Biol. 2010;10. doi:10.1186/1472-6769-10-6

241. Buser R, Kellner V, Melnik A, et al. The Replisome-Coupled E3 Ubiquitin Ligase Rtt101Mms22 Counteracts Mrc1 Function to Tolerate Genotoxic Stress. Campbell 
JL, ed. PLoS Genet. 2016;12(2):e1005843. doi:10.1371/journal.pgen.1005843

242. Onge RPS, Mani R, Oh J, et al. Systematic pathway analysis using high-resolution fitness profiling of combinatorial gene deletions. Nat Genet. 2007;39(2):199-206. doi:10.1038/ng1948

243. Kawashima S, Ogiwara H, Tada S, et al. The INO80 complex is required for damage-induced recombination. Biochem Biophys Res Commun. 2007;355(3):835-841. doi:10.1016/J.BBRC.2007.02.036

244. Bird AW, Yu DY, Pray-Grant MG, et al. Acetylation of histone H4 by Esa1 is required for DNA double-strand break repair. Nature. 2002;419(6905):411-415. doi:10.1038/nature01035

245. Tsukuda T, Fleming AB, Nickoloff JA, Osley MA. Chromatin remodelling at a DNA double-strand break site in Saccharomyces cerevisiae. Nature. 2005;438(7066):379-383. doi:10.1038/nature04148

246. Chambers AL, Downs JA. The RSC and INO80 chromatin-remodeling complexes in DNA double-strand break repair. Prog Mol Biol Trans/ Sci. 2012;110:229-261. doi:10.1016/B978-0-12-387665-2.00009-2

247. Li J, Summerlin M, Nitiss KC, Nitiss JL, Hanakahi LA. TDP1 is required for efficient non-homologous end joining in human cells. DNA Repair (Amst). 2017;60:40-49. doi:10.1016/j.dnarep.2017.10.003 
248. Zhiyong M, Michael B, Andrei S, Vera G. DNA repair by nonhomologous end joining and homologous recombination during cell cycle in human cells. Cell Cycle. 2009;7(18):2902-2906.

249. Ceccaldi R, Rondinelli B, Andrea ADD. Repair pathway choices and consequences at the DSB. Trends Cell Biol. 2016;26(1):52-64. doi:10.1016/j.tcb.2015.07.009

250. Mathiasen DP, Lisby M. Cell cycle regulation of homologous recombination in Saccharomyces cerevisiae. FEMS Microbiol Rev. 2014. doi:10.1111/15746976.12066

251. Somesh BP, Reid J, Liu W-F, et al. Multiple mechanisms confining RNA polymerase II ubiquitylation to polymerases undergoing transcriptional arrest. Cell. 2005;121(6):913-923. doi:10.1016/j.cell.2005.04.010

252. Daraba A, Gali VK, Halmai M, Haracska L, Unk I. Def1 Promotes the Degradation of Pol3 for Polymerase Exchange to Occur During DNA-Damage-Induced Mutagenesis in Saccharomyces cerevisiae. PLoS Biol. 2014. doi:10.1371/journal.pbio.1001771

253. Arnoult N, Correia A, Ma J, et al. Regulation of DNA repair pathway choice in S and G2 phases by the NHEJ inhibitor CYREN. 2017. doi:10.1038/nature24023

254. Yu X, Gabriel A. Ku-dependent and Ku-independent end-joining pathways lead to chromosomal rearrangements during double-strand break repair in Saccharomyces cerevisiae. Genetics. 2003;163(3):843-856. 
http://www.ncbi.nlm.nih.gov/pubmed/12663527. Accessed July 18, 2019.

255. Sinha S, Li F, Villarreal D, et al. Microhomology-mediated end joining induces hypermutagenesis at breakpoint junctions. PLoS Genet. 2017. doi:10.1371/journal.pgen.1006714

256. McVey M, Lee SE. MMEJ repair of double-strand breaks (director's cut): deleted sequences and alternative endings. Trends Genet. 2008. doi:10.1016/j.tig.2008.08.007

257. Berger B, Peng J, Singh M. Computational solutions for omics data. Nat Rev Genet. 2013. doi:10.1038/nrg3433

258. Zhou C, Elia AEH, Naylor ML, et al. Profiling DNA damage-induced phosphorylation in budding yeast reveals diverse signaling networks. Proc Natl Acad Sci. 2016. doi:10.1073/pnas.1602827113

259. Jazayeri A, Jackson SP. Screening the yeast genome for new DNA-repair genes. Genome Biol. 2002;3(4):REVIEWS1009. doi:10.1186/gb-2002-3-4-reviews1009

260. Hendry JA, Tan G, Ou J, Boone C, Brown GW. Leveraging DNA Damage Response Signaling to Identify Yeast Genes Controlling Genome Stability. G3\&amp;\#58; Genes/Genomes/Genetics. 2015. doi:10.1534/g3.115.016576

261. Panday A, Xiao L, Gupta A, Grove A. Control of DNA end resection by yeast Hmo1p affects efficiency of DNA end-joining. DNA Repair (Amst). 2017. 
doi:10.1016/j.dnarep.2017.03.002

262. Murray JE, Van Der Burg M, ljspeert $\mathrm{H}$, et al. Mutations in the NHEJ component XRCC4 cause primordial dwarfism. Am J Hum Genet. 2015;96(3):412-424. doi:10.1016/j.ajhg.2015.01.013

263. Ferguson LR, Chen $\mathrm{H}$, Collins $A R$, et al. Genomic instability in human cancer: Molecular insights and opportunities for therapeutic attack and prevention through diet and nutrition. Semin Cancer Biol. 2015;35 Suppl(Suppl):S5-S24. doi:10.1016/j.semcancer.2015.03.005

264. Srivastava M, Raghavan SC. DNA double-strand break repair inhibitors as cancer therapeutics. Chem Biol. 2015. doi:10.1016/j.chembiol.2014.11.013

265. Pandey M, Gopalakrishnan V, Swarup H, et al. Water-soluble version of SCR7pyrazine inhibits DNA repair and abrogates tumor cell proliferation. J Radiat Cancer Res. 2019. doi:10.4103/jrcr.jrcr_24_18

266. White RR, Vijg J. Do DNA Double-Strand Breaks Drive Aging? Mol Cell. 2016. doi:10.1016/j.molcel.2016.08.004

267. Guo T, Feng Y-L, Xiao J-J, et al. Harnessing accurate non-homologous end joining for efficient precise deletion in CRISPR/Cas9-mediated genome editing. Genome Biol. 2018;19(1):170. doi:10.1186/s13059-018-1518-x

268. Wang G, Zhao N, Berkhout B, Das AT. CRISPR-Cas9 can inhibit HIV-1 replication 
but NHEJ repair facilitates virus escape. Mol Ther. 2016;24(3):522-526.

doi:10.1038/mt.2016.24

\section{Appendix}

\section{Appendix 8.1: Additional data for chapter 2}

BlastP analysis of anti-target proteins with closest yeast homolog shown, related to Table 1. Closest yeast homolog of anti-Psk1 (A), anti-Pin4 (B) and anti-Rmd1 (C) are shown. 


\section{A Anti-Psk1}

- Mmp1p [Saccharomyces cerevisiae CEN.PK113-7D]

$\begin{array}{llllll}\text { Score } & \text { Expect } & \text { Method } & \text { Identities } & \text { Positives } & \text { Gaps } \\ 36.2 \text { bits(82) } & 0.015 & \text { Compositional matrix adjust. } & 14 / 27(52 \%) & 18 / 27(66 \%) & 0 / 27(0 \%) \\ \text { Anti PSk1 } & 65 & \begin{array}{l}\text { MAQCAPEEACQYPVRRYGLHATNCIE } \\ + \text { QC E +CQYPV SY LHA+ I+ }\end{array} & \\ \text { MMP1 } & 120 & \text { VVQCGAESCQYPVGSYLHASRFID } & 146 & \end{array}$

\section{B Anti-Pin4}

- Esl1p [Saccharomyces cerevisiae S288c]

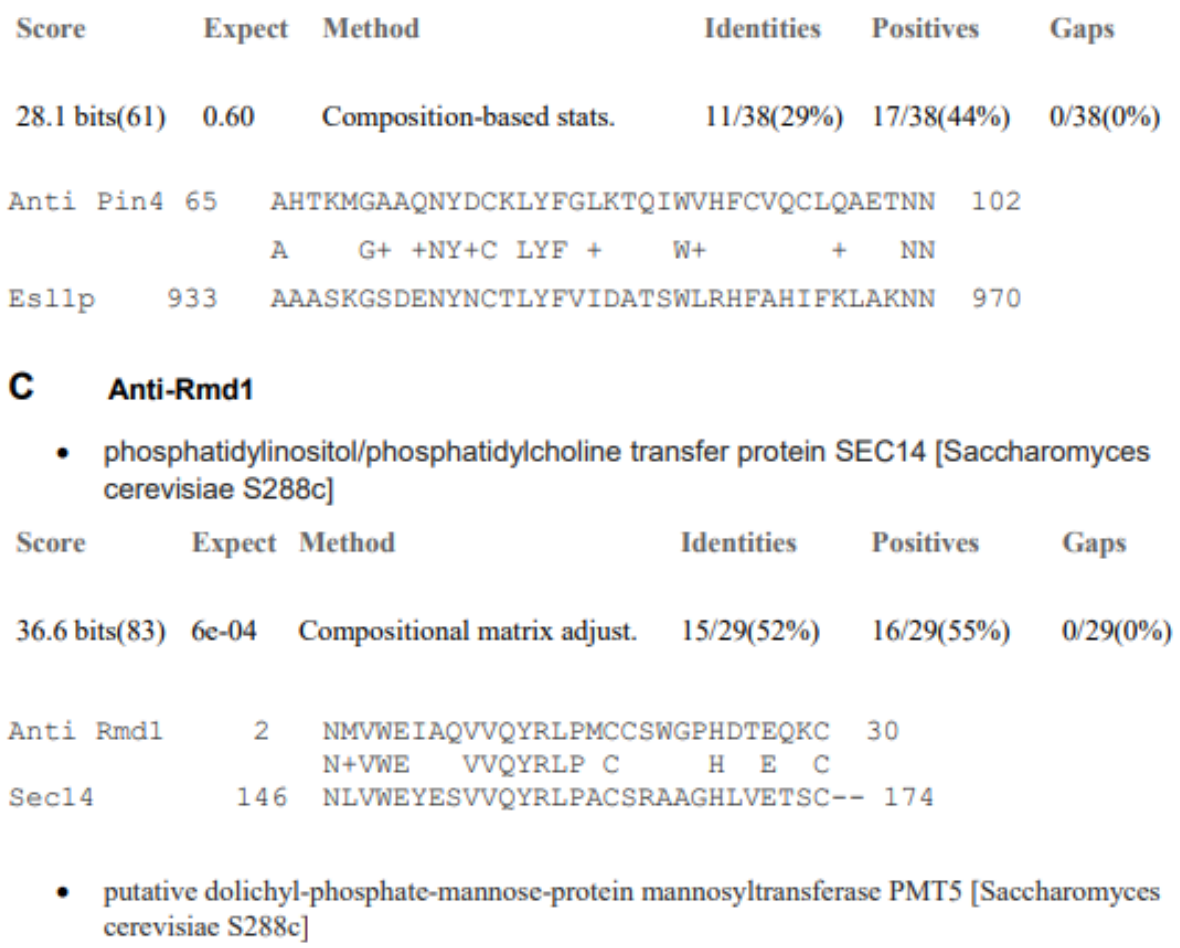

\section{InSiPS Output for all 18 candidate sequences}




\begin{tabular}{llrrrr}
\hline $\begin{array}{l}\text { Target } \\
\text { name }\end{array}$ & $\begin{array}{l}\text { Systematic } \\
\text { gene name }\end{array}$ & \multicolumn{1}{c}{ Fitness } & \multicolumn{1}{l}{$\begin{array}{l}\text { Target } \\
\text { score }\end{array}$} & \multicolumn{1}{l}{$\begin{array}{l}\text { Max non- } \\
\text { target score }\end{array}$} & \multicolumn{1}{l}{$\begin{array}{l}\text { Average non-target } \\
\text { Score }\end{array}$} \\
\hline REI1 & YBR267W & 0.437109 & 0.66573458 & 0.34341903 & 0.03132127 \\
CHK1 & YBR274W & 0.570826 & 0.70807537 & 0.19383371 & 0.019953153 \\
BDH1 & YAL060W & 0.290459 & 0.54265767 & 0.4647474 & 0.236912524 \\
AIM2 & YAL049C & 0.276894 & 0.5333423 & 0.48083291 & 0.139402474 \\
HSM3 & YBR272C & 0.358195 & 0.59402891 & 0.39700788 & 0.125126232 \\
ACS1 & YAL054C & 0.45144 & 0.64881096 & 0.3042048 & 0.015965355 \\
RPL19B & YBL027W & 0.329424 & 0.54180512 & 0.39198845 & 0.076419325 \\
PIN4 & YBL051C & 0.313386 & 0.55347628 & 0.43378601 & 0.144189497 \\
PSK1 & YAL017W & 0.541232 & 0.71515927 & 0.24320134 & 0.041448462 \\
RMD1 & YDL001W & 0.399594 & 0.67404023 & 0.4071663 & 0.088788771 \\
PSK2 & YOL054W & 0.433372 & 0.65443715 & 0.33779394 & 0.025854975 \\
HEK2 & YBL032W & 0.66108 & 0.84123403 & 0.21415452 & 0.018977927 \\
CCR4 & YAL021C & 0.464692 & 0.67289423 & 0.30941335 & 0.059046125 \\
CDC24 & YAL041W & 0.364046 & 0.62862367 & 0.4208845 & 0.114832926 \\
FUN11 & YAL036C & 0.301512 & 0.58082879 & 0.48089416 & 0.079736457 \\
YPT10 & YBR264C & 0.283617 & 0.54491094 & 0.47951721 & 0.265006925 \\
EFM2 & YBR271W & 0.22569 & 0.4165458 & 0.45818566 & 0.291757241 \\
PRD1 & YCL057W & 0.24819 & 0.45788308 & 0.45796182 & 0.315611118 \\
\hline
\end{tabular}

\section{Sequences of anti-target proteins against all 18 original targets}




\begin{tabular}{|c|c|}
\hline $\begin{array}{l}\text { Target } \\
\text { Protein }\end{array}$ & Sequence anti-target protein \\
\hline REl1 & $\begin{array}{l}\text { HHHHHHVMKPAEFAVQAIYKLAPVARRMHCYVAWTDSQNTIISRNAINGVIHADAHDCGVSNPNFGD } \\
\text { SARFGNGQVCIVCNEQMKLLTKHPWNVVQHRNMGCPMKTEMCERMPGYMEPCFHQFNPASMPDD } \\
\text { SDDWRDAQCEPAGKINGL }\end{array}$ \\
\hline CHK1 & $\begin{array}{l}\text { HHHHHHAHFIHGSPLAIEAIGAALAYGCKFMFIHAPLYLCYSDWRVTGMDFHDMKEPAWAHVSAGVPY } \\
\text { SAPAPCYHTYSIMMVGYLAGKHIFANREPWPWYVPSRTHAGQRVFPTDAPIWEDDQRERMGAAQQ } \\
\text { SKWIGRRPDKMSGKAF }\end{array}$ \\
\hline $\mathrm{BDH} 1$ & $\begin{array}{l}\text { HHHHHHQNQQRACQPMPQHQQQHHAADQLQARYRWQLEPWHEWAWCAACARGYYETHVVCITCF } \\
\text { CIGMAKPHISYTERHERPSKCAAWSRDALTAKFARTAKASKCACNLYMHCPLYRNCPQAQTCPIYRCD } \\
\text { ELGAVKTHFCAEAWETSW }\end{array}$ \\
\hline AlM2 & $\begin{array}{l}\text { HHHHHHSATQADSTAHESTKFHNSKNADQPHTCYFPMMCHGAYFEGWPPCCKWYLQHNEHPGHYR } \\
\text { THAHIDHCTSNETRKKKHHQHHIKKYRQMHTDGIRCSWRNKDESKTGPVTPVRLKNAHSKSCYGFCH } \\
\text { FNNQMQPCSNYKRPSSGN }\end{array}$ \\
\hline HSM3 & $\begin{array}{l}\text { HHHHHHQQAGMQSQQQQQEQLCKEEPQTKAGPEYESNHAFAQQQVNMCAHGKCAAFWQTKNDWI } \\
\text { QMFDDPTTRCSPCHWAADPAEVQEEAVAADIKIWHCDKQCQCCSRMSDGIPHRIVCCFYTDDSRYAK } \\
\text { FKSVYDGFGFTSMMIVPCS }\end{array}$ \\
\hline ACS1 & $\begin{array}{l}\text { HHHHHHIFIWISDKVSWMNCRFAGDKQNAHTYSCMHFQEPMSEMWLKLLMYTARFANTEPRGVYTH } \\
\text { EARPVWTNIKDYVFNAAICPAWREKGGNNAANSMENHYVGACAVESNECIKPHCVPALWFYCKFCHQ } \\
\text { FTEPHMHPSAYILAKGT }\end{array}$ \\
\hline RPL19B & $\begin{array}{l}\text { HHHHHHRCPPHSRKGQLLFRHAFADREQQGRNQWPYQNRNWKARYEACPKKEMQVTVKLLWCEM } \\
\text { NMAWHCQQAIRGRHHIISHNQLWHDEEEFGIPCAMEVNQLRIHFNWLFSLYVPFGGLMPPHLAFQNR } \\
\text { MHCLEKEGTKFIPAMEYFA }\end{array}$ \\
\hline PIN4 & $\begin{array}{l}\text { HHHHHHTMACYQWRQMHERQHHHRENKTVAAVEMCHCLMTWAYHKARWDCRECGQTGVPFHQW } \\
\text { QHHQQQARQHSQKHRAALYREDMMFFHFYNHPNHLVHQVTQMTQYWAHFIPRSHPWEPATCQSNIL } \\
\text { VCCKNTCYTMAHTVRTTDAEW }\end{array}$ \\
\hline PSK1 & $\begin{array}{l}\text { HHHHHHEQVHRQVHDKERAQAAVGTWLFALCDLCSYGGWFCCCFCRKRVRILVFAFKHHRICRREW } \\
\text { WRWCTKKMNIHMIPTQVVMVMIEPHIMALYMGCQTGFIECDFYMGRFRSQAHFVHMFFAQMVWQAK } \\
\text { CTQQDWYSRINRKKNDHL }\end{array}$ \\
\hline RMD1 & $\begin{array}{l}\text { HHHHHHHCECDQPVHAIAYQLYVLVEFCDHGAMEKYMMTRWHAALREVLGEICTDWGGDYTQAHAQ } \\
\text { HAEAEAECMMHGIAAHPYFIIQWPTFHDQEQTYNIDDTGAGACCKNHAAEHTCKDEPEWPKNFRWG } \\
\text { QFAGPIHCCQGYADTAQI }\end{array}$ \\
\hline PSK2 & $\begin{array}{l}\text { HHHHHHATQCDPQYFQQDAPPNQGELCKWQIHVYFRFQAYWERPHHAEETHAAWRRWWVQNKTM } \\
\text { KVVLPKMHRGVGKSGEHRLMMWALECQYVMTARWFAKFGGTKAGMIFIMKRNYKLRCHPCFEIWAT } \\
\text { AKNRMPWNFPGQGGSDSVTV }\end{array}$ \\
\hline HEK2 & $\begin{array}{l}\text { HHHHHHSVKANCSYFWAKYNYILTAWWATNEAERHTRRAEWKDFYHWGLIKAPVSVSINPECTTANE } \\
\text { EYFPWWFLYKNRAGQYPTGPPAMAPHAKSPQTMQSKQAEIGMMHPNVFAREWHHYAPPQNEMFMS } \\
\text { ARFLMQGKTPCQYGGRIR }\end{array}$ \\
\hline CCR4 & $\begin{array}{l}\text { HHHHHHFYCTHLQDRWAMIGWGCLPPHNCTGFAENEDEFPLAISLKNKYRKVKFCFLLYCLCTCPIEY } \\
\text { AFTGHEQIAARGQQRSHQTQYYKPGMHVGWRKSPTKFFLYYKQPGRLCQGMTCVMVMQEQHQENL } \\
\text { DWQQKEKHRKASSQQYD }\end{array}$ \\
\hline CDC24 & $\begin{array}{l}\text { HHHHHHSMARCFESYMDNVMRPDRIWHELWYAGVNDMWAAFQCCPWCTWFQCEVHYLYVITCALK } \\
\text { VARCWEAVDHYWPFVTAAWAQQLAVPFMQEQQHLQTHDQEDDLMHQKLIKICWLKNAQHPGAFPAL } \\
\text { AECFCYWKPWVNTLSVAL }\end{array}$ \\
\hline FUN11 & $\begin{array}{l}\text { HHHHHHSHAPYHKQASGDRHPSIRFWANMQLFLFCESYQKAACSAWCFPLVCTYNEAMNAWVRVP } \\
\text { EIFLHRPWYPNILATRDPCNYYTNFLGSSRLLVNCGRRQNWWWEEEGAERCHPMMSAHLVDTHRPI } \\
\text { YGQSGQVHHCSDDNDNG }\end{array}$ \\
\hline YPT10 & $\begin{array}{l}\text { HHHHHHFLMARAAFNYWYMGYPPIFITIQAVIQEENGDQHFQQQEHGQPGEQTAQMQQHQQPEQQH } \\
\text { MKTEMAACPSDGLGAACPTWFHEGTGCPRCKFPARGQIFAKCHRTWMQWDMFVLQWAYTLEFWQ } \\
\text { CSGEHDMCLFFRNICHAVAY }\end{array}$ \\
\hline EFM2 & $\begin{array}{l}\text { HHHHHHIPAQCKLWSTLTAKQCFNCGQTNGTRCYRCGQFCHKIRDCPEARDCYHCGNGALVFCYWK } \\
\text { NNCHERAFRYACPWLPTADVGAPWKCPDGGSQCEHNCDAAWLTWAVQSIQTHNQPKQGPQQQEQ } \\
\text { RNQNERQQSHVKQTTGQKEY }\end{array}$ \\
\hline PRD1 & $\begin{array}{l}\text { HHHHHHAYDDYMYHDHFHNNHEHPHQLMCKLRDYAHYLTWCERQGQAQCLCDARVDVRQRMEYC } \\
\text { PFFEGYREQCKFGPHCTQSACSYRPPTHQCKCNCACQGCPEAQCCTCHSYSQDGGGSTQQQEPPH } \\
\text { HQPAQWQQQDPNTQVDNAPYE }\end{array}$ \\
\hline
\end{tabular}




\section{Primers used for $\mathrm{Y} 2 \mathrm{H}$ cloning}

F-primer: Psk1 5’ AGC ACC $\downarrow$ TCG AGC CCC TAC ATC GGT GCT TCC AAC CTC TCA GAA CAT

R-primer: Psk1 5’ ACT GAG CGG CCG CCA TCA TCA AAT AAC CAA CCA TTT GTC GTT ATT

F-primer: Pin4 5’ AGC ACC TCG AGC GAG ACC AGT TCT TTT GAG AAT GCT CCT CCT GCA

R-primer: Pin4 5’ ACT GAG CGG CCG CCA TTA TTA CCA TAG ATT CTT CTT GTT TTG GTT

F-primer: Anti-Pin4 5’ AGC AGC AGC AGC AGC AGC AGC AGC AGC ACC TCG AGC ATT TTC ATC TAC GGT GAT AGA TTA TTG GAT CAA

R-primer: Anti-Pin4 5' ACT AGC GGC CGC TTA TTA ATG ATG GTG ATG GTG GTG ATG ATG GTG ATG ATG ATG GGC

F-primer: Anti-Psk1 5’ AGC AGC AGC AGC AGC AGC ACC $\downarrow$ TCG AGC TCT GAT AAT GAA CAC TTG CAT AAG TGC CAA

R-primer: Anti-Psk1 5’ ACT AGC $\downarrow$ GGC CGC TTA TTA ATG ATG GTG ATG GTG GTG ATG ATG GTG ATG ATG ATG CTG 


\section{Anti-pin4 protein sensitizes cells to arsenite exposure}

Drug sensitivity growth curve of the anti-Pin4 protein causes sensitivity to arsenite

(0.1M) similar to $\Delta$ pin4, related to Figure 2 . A single large culture $(40 \mathrm{~mL})$ of each condition is represented but multiple repeats demonstrated the same trend.

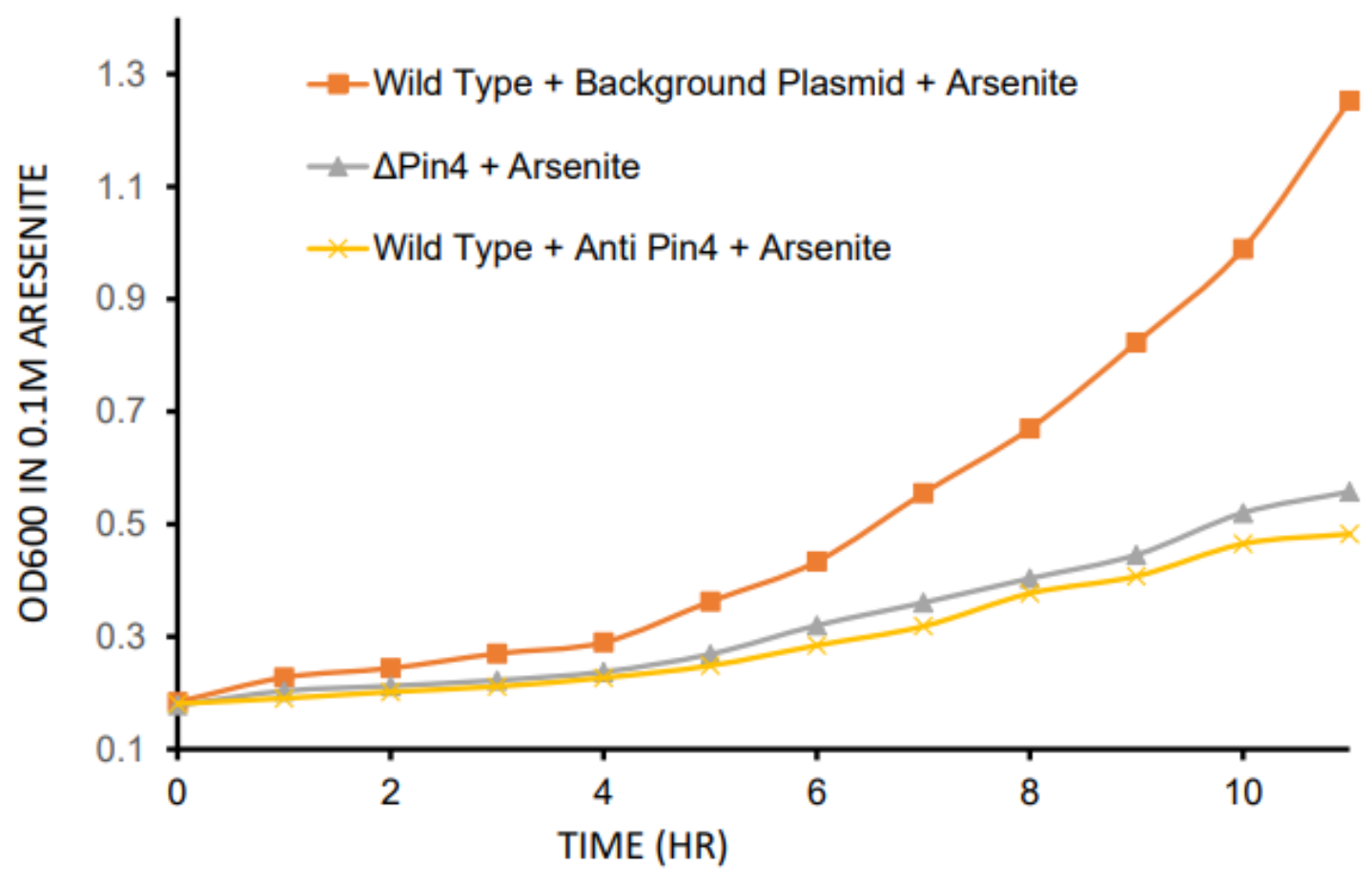




\section{Appendix 8.2: Additional Data for Chapter 3}

Table 8.1 Genetic compensation analysis of the overexpression of PSK1 in various mutant strains of interest. Constructs were exposed to conditions of UV light exposure (30 sec), $0.04 \% \mathrm{v} / \mathrm{v} \mathrm{MMS}$, or $70 \mathrm{mM}$ hydroxyurea. Cultures were grown to saturation and then serially diluted and spot-tested on YPGal or YPGal + Drug (or UV). Visually analysis of three repeats assessed growth relative to WT from 1-4.

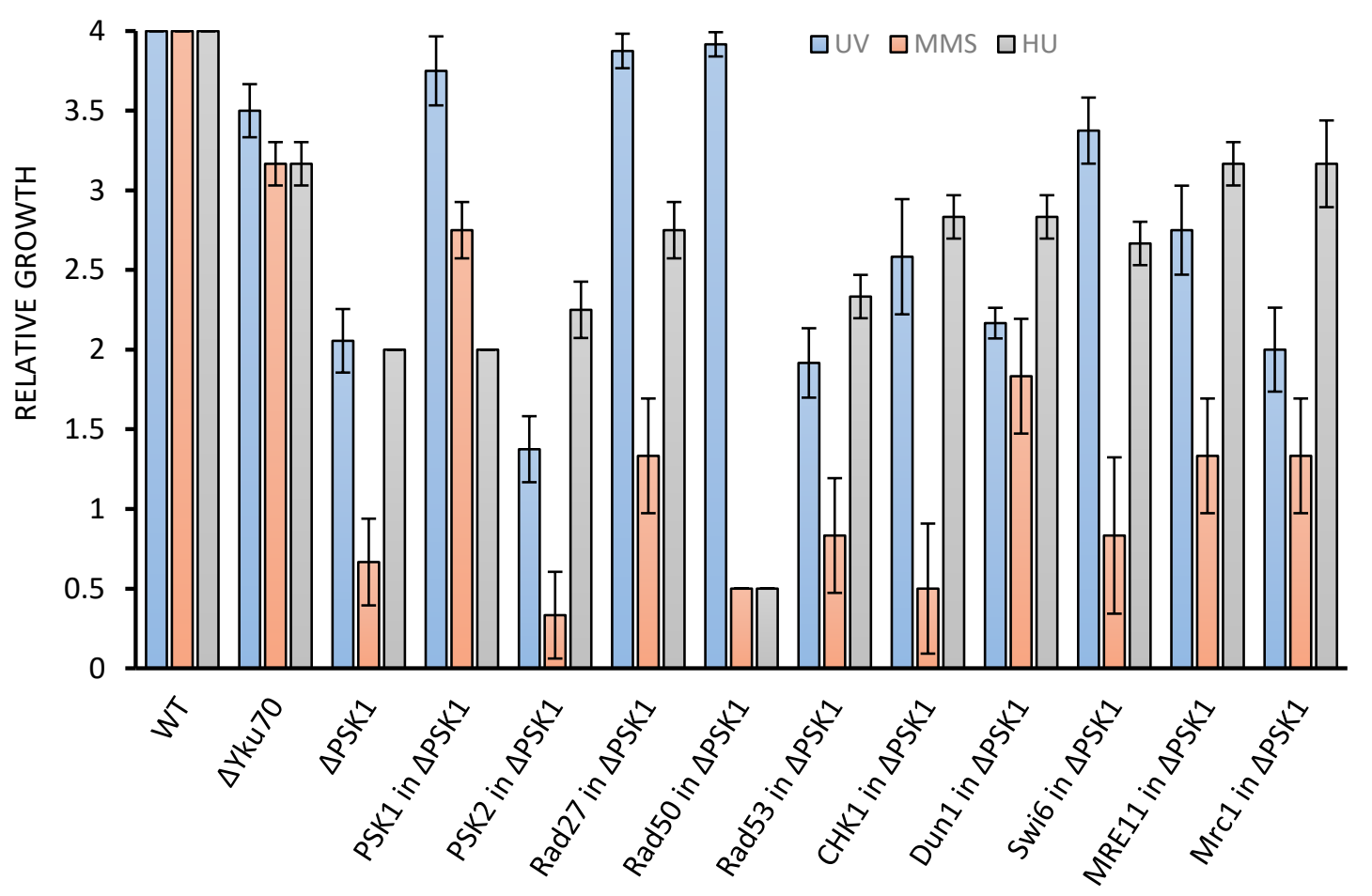




\section{Appendix 8.3: Selected Publications}

In Silico Engineering of Synthetic Binding Proteins from Random Amino Acid

\section{Sequences.}

Burnside D, Schoenrock A, Moteshareie H, Hooshyar M, Basra P, Hajikarimlou M, Dick K, Barnes B, Kazmirchuk T, Jessulat M, Pitre S, Samanfar B, Babu M, Green JR, Wong A, Dehne F, Biggar KK, Golshani A.

iScience. 2019 Jan 25;11:375-387. doi: 10.1016/j.isci.2018

iScience

CellPress

\section{Article}

In Silico Engineering of Synthetic Binding Proteins from Random Amino Acid Sequences

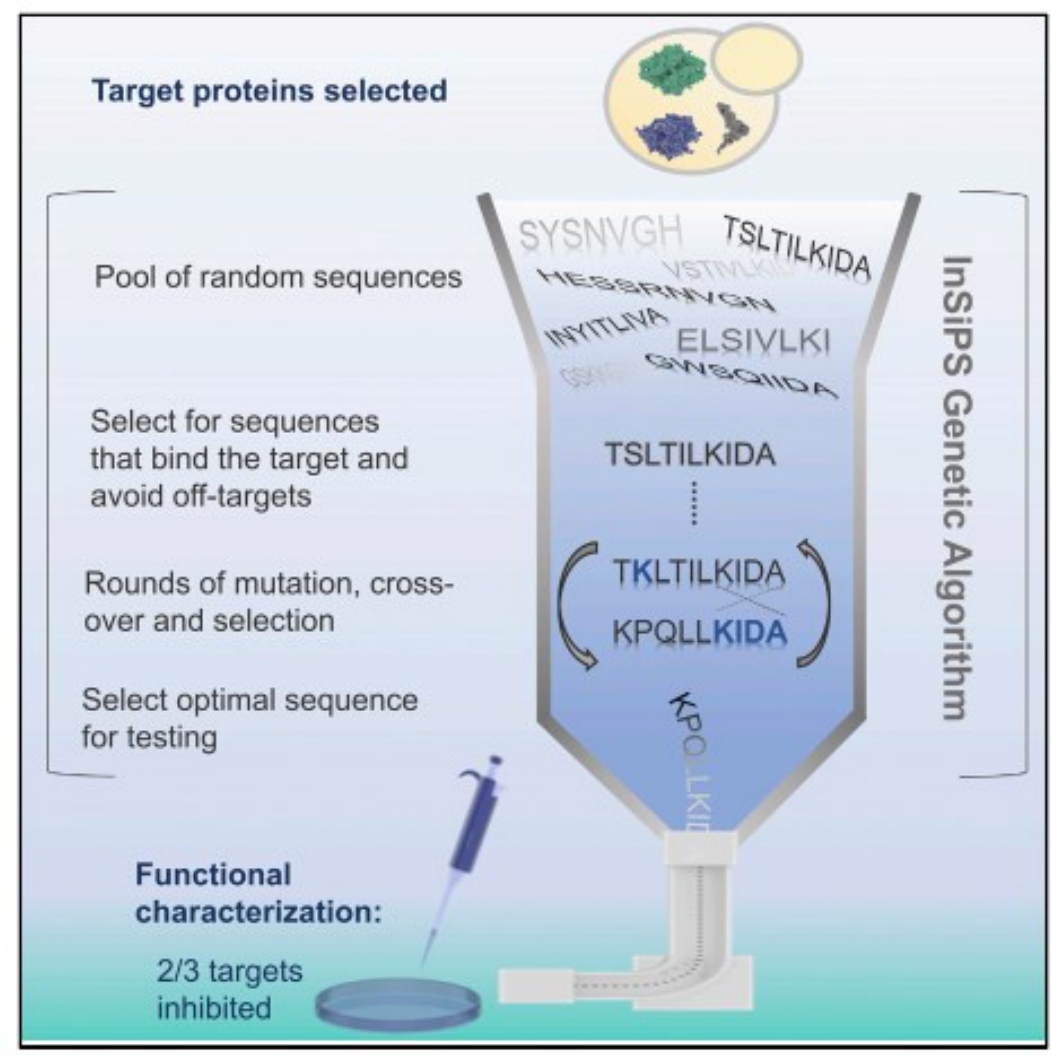

Daniel Burnside,

Andrew

Schoenrock,

Houman

Moteshareie, ....

Frank Dehne, Kyle

K. Biggar, Ashkan

Golshani

ashian-golshaniacarletances

HIGHLGHTS

InSiPS engineers synthetic

binding proteins ( $\left(\mathrm{SP}_{\mathrm{s}}\right)$

using primary protein

sequence

SBPs are designed to a

bind a target protein and

avoid "offtetarger"

interactions

Binding and functional

inhibition of two of three

target proteins in yeast is

demonstrated

Our new approsch offers

advantages over

alternative tools that rely on 3D models 


\title{
Evolution of protein-protein interaction networks in yeast.
}

\author{
Schoenrock A, Burnside D, Moteshareie H, Pitre S, Hooshyar M, Green JR, Golshani A, \\ Dehne $F$, Wong $A$.
}

PLoS One. 2017 Mar 1;12(3):e0171920. doi: 10.1371/journal.pone.0171920

\section{:PLOS | one}

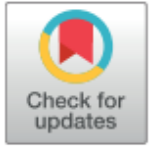

\section{fopen access}

Citation: Schoenrock A, Burnside D, Moteshareie H, Pitre S, Hooshyar M, Green JR, et al. (2017) Evolution of protein-protein interaction networks in yeast. PLOS ONE 12(3): e0171920. doi:10.1371/ journal.pone.0171920

Editor: Franca Fraternali, King's College London, UNITED KINGDOM

Received: May 9, 2016

Accepted: January 28, 2017

Published: March 1, 2017

\section{RESEARCH ARTICLE}

\section{Evolution of protein-protein interaction networks in yeast}

Andrew Schoenrock', Daniel Burnside ${ }^{2}$, Houman Moteshareie ${ }^{2}$, Sylvain Pitre ${ }^{1}$, Mohsen Hooshyar ${ }^{2}$, James R. Green ${ }^{3}$, Ashkan Golshani ${ }^{2}$, Frank Dehne', Alex Wong ${ }^{2 *}$

1 School of Computer Science, Carleton University, Ottawa, Canada, 2 Department of Biology, Carleton University, Ottawa, Canada, 3 Department of Systems and Computer Engineering, Carleton University, Ottawa, Canada

* alex.wong@carleton.ca

\begin{abstract}
Interest in the evolution of protein-protein and genetic interaction networks has been rising in recent years, but the lack of large-scale high quality comparative datasets has acted as a barrier. Here, we carried out a comparative analysis of computationally predicted proteinprotein interaction (PPI) networks from five closely related yeast species. We used the Protein-protein Interaction Prediction Engine (PIPE), which uses a database of known interactions to make sequence-based PPI predictions, to generate high quality predicted interactomes. Simulated proteomes and corresponding PPI networks were used to provide null expectations for the extent and nature of PPI network evolution. We found strong evidence for conservation of PPIs, with lower than expected levels of change in PPIs for about a quarter of the proteome. Furthermore, we found that changes in predicted PPI networks are poorly predicted by sequence divergence. Our analyses identified a number of functional classes experiencing fewer PPI changes than expected, suggestive of purifying selection on PPIs. Our results demonstrate the added benefit of considering predicted PPI networks when studying the evolution of closely related organisms.
\end{abstract}


Use of chemical genomics to investigate the mechanism of action for inhibitory bioactive natural compounds.

Burnside, D, Moteshareie, H, Marquez, I.G., Hooshyar, M, Samanfar, B, Shostak, K, Omidi, K, Peery, H.E., Smith, M.L. and Golshani, A.

Bioactive Natural Products. 2015. Wiley-VCH, 9-31

Use of Chemical Genomics to Investigate the Mechanism of Action for Inhibitory Bioactive Natural Compounds ${ }^{1}$

Daniel Burnside, Houman Moteshareie, Imelda G. Marquez, Mohsen Hooshyar,

Bahram Samanfar, Kristina Shostak, Katoyoun Omidi, Harry E. Peery, Myron L. Smith, and

Ashkan Golshani

\section{WILEY-VCH}

Edited by Goutam Brahmachari

\section{Bioactive \\ Natural Products}

Chemistry and Biology

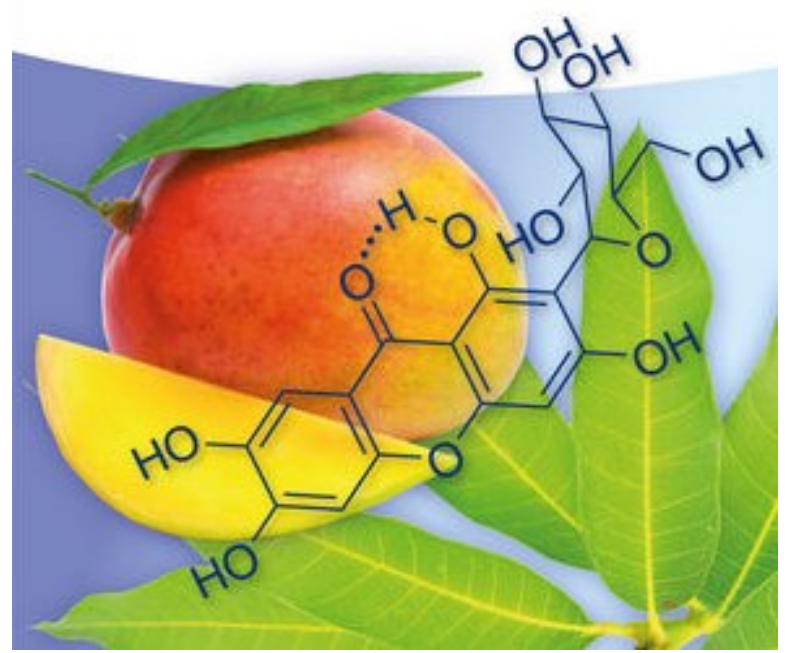




\section{Designing anti-Zika virus peptides derived from predicted human-Zika virus protein- protein interactions.}

Kazmirchuk T, Dick K, Burnside D, Barnes B, Moteshareie H, Hajikarimlou M, Omidi K, Ahmed D, Low A, Lettl C, Hooshyar M, Schoenrock A, Pitre S, Babu M, Cassol E, Samanfar B, Wong A, Dehne F, Green JR, Golshani A.

Comput Biol Chem. 2017 Dec;71:180-187. doi: 10.1016/j.compbiolchem.2017.10.011.

\begin{tabular}{|c|c|c|}
\hline$\frac{1}{2}$ & $\begin{array}{l}\text { Contents lists available at ScienceDirect } \\
\text { Computational Biology and Chemistry }\end{array}$ & 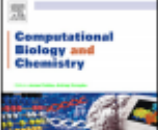 \\
\hline$\overline{\text { FI SFVIFR }}$ & journal homepage: www.elsevier.com/locate/compbiolchem & $-1-=$ \\
\hline
\end{tabular}

Research article

Designing anti-Zika virus peptides derived from predicted human-Zika virus protein-protein interactions

Tom Kazmirchuk ${ }^{\mathrm{a}}$, Kevin Dick ${ }^{\mathrm{b}}$, Daniel. J. Burnside ${ }^{\mathrm{a}}$, Brad Barnes ${ }^{\mathrm{b}}$,

Houman Moteshareie ${ }^{\mathrm{a}}$, Maryam Hajikarimlou ${ }^{\mathrm{a}}$, Katayoun Omidia ${ }^{\mathrm{a}}$, Duale Ahmed ${ }^{\mathrm{a}, \mathrm{c}}$,

Andrew Low ${ }^{\mathrm{a}}$, Clara Lettl ${ }^{\mathrm{a}}$, Mohsen Hooshyar ${ }^{\mathrm{a}}$, Andrew Schoenrock ${ }^{\mathrm{d}}$, Sylvain Pitre ${ }^{\mathrm{d}}$,

Mohan Babu ${ }^{e}$, Edana Cassol ${ }^{c}$, Bahram Samanfarf, Alex Wong ${ }^{a}$, Frank Dehne ${ }^{c}$,

James. R. Green ${ }^{\mathrm{b}}$, Ashkan Golshani ${ }^{\mathrm{a}, *}$

"Department of Biology and Institute of Biochemistry, and Ottawa Institute of Systems Biology, Carleton University, Ottawa, Ontaria. K1S 5B6, Canado

'Department of Systems and Computer Engineering, Carleton University, Ottawa, Ontaria. K1S 5B6, Canada

'Department of Health Science, Carleton University, Ottawa, Ontaria. K1S 5B6, Canodo

' School of Computer Science, Carleton University, Ottawa, Ontario. K1S 5B6, Canada

- Department of Biochemistry, University of Regina, Regina, Saskatchewan SAS OA2, Conada

'Agriculture and Agri-Food Canada, Ottawa Research and Development Centre (ORDC), Ottawa, Ontario. K1A OC6, Canada

A RTICLE INFO

Article history:

Received 18 August 2017

Received in revised form 3 October 2017

Accepted 27 October 2017

Available online 2 November 2017

Keywords:

Synthetic peptide design

Synthetic peptice d

Anti-Zika virus peptides

In silico drug design

Protein-protein interaction prediction

Host-virus interactions
A B S T R A C T

The production of anti-Zika virus (ZIKV) therapeutics has become increasingly important as the propagation of the devastating virus continues largely unchecked. Notably, a causal relationship between ZIKV infection and neurodevelopmental abnormalities has been widely reported, yet a specific mechanism underlying impaired neurological development has not been identified. Here, we report on the design of several synthetic competitive inhibitory peptides against key pathogenic ZIKV proteins through the prediction of protein-protein interactions (PPIs). Often, PPIs between host and viral proteins are crucial for infection and pathogenesis, making them attractive targets for therapeutics. Using two complementary sequence-based PPI prediction tools, we first produced a comprehensive map of predicted human-ZIKV PPIs (involving 209 human protein candidates). We then designed several peptides intended to disrupt the corresponding host-pathogen interactions thereby acting as anti-ZIKV therapeutics. The data generated in this study constitute a foundational resource to aid in the multidisciplinary effort to combat ZIKV infection, including the design of additional synthetic proteins.

2017 Published by Elsevier Ltd. 
Manganese-induced cellular disturbance in the baker's yeast, Saccharomyces cerevisiae with putative implications in neuronal dysfunction.

Hernández RB, Moteshareie H, Burnside D, McKay B, Golshani A.

Sci Rep. 2019 Apr 25;9(1):6563. doi: 10.1038/s41598-019-42907-2.

\section{SCIENTIFIC REPORTS}

Article | OPEN | Published: 25 April 2019

Manganese-induced cellular disturbance in the baker's yeast, Saccharomyces cerevisiae with putative implications in neuronal dysfunction

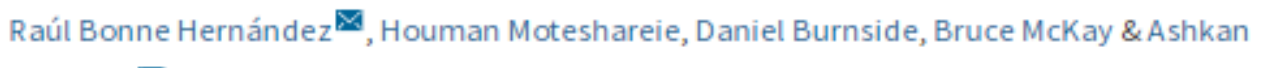
Golshani

Scientific Reports 9, Article number: 6563 (2019) | Download Citation $\underline{\Perp}$ 
Uncharacterized ORF HUR1 influences the efficiency of non-homologous end-joining repair in Saccharomyces cerevisiae.

Omidi K, Jessulat M, Hooshyar M, Burnside D, Schoenrock A, Kazmirchuk T, Hajikarimlou M, Daniel M, Moteshareie H, Bhojoo U, Sanders M, Ramotar D, Dehne F, Samanfar B, Babu M, Golshani A.

Gene. 2018 Jan 10;639:128-136. doi: 10.1016/j.gene.2017.10.003.

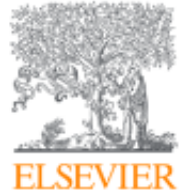

Gene

Volume 639, 10 January 2018, Pages 128-136

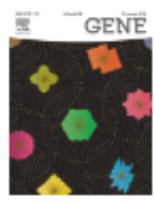

Research paper

Uncharacterized ORF HUR1 influences the efficiency of non-homologous end-joining repair in Saccharomyces cerevisiae

Katayoun Omidi a, b, Matthew Jessulat c, Mohsen Hooshyar ${ }^{a, b}$, Daniel Burnside ${ }^{a, b}$, Andrew Schoenrock ${ }^{d}$, Tom Kazmirchuk a, b, Maryam Hajikarimlou a, b, Mary Daniel a, b, Houman Moteshareie a, b, Urvi Bhojoo a, b, Megan Sanders ${ }^{3,}$, , Dindial Ramotar ${ }^{e}$, Frank Dehne ${ }^{d}$, Bahram Samanfar ${ }^{f}$, Mohan Babu c, Ashkan Golshani a, b $2 \otimes$ 


\section{Engineering inhibitory proteins with InSiPS: The in-silico protein synthesizer}

Schoenrock, A, Burnside, D, Moteshareie M, Wong A, Golshani A, Dehne, F.

Proceedings of the International Conference for High Performance Computing, Networking, Storage and Analysis. 2015. p. 25. ACM.

\section{Engineering Inhibitory Proteins with InSiPS: The In-Silico Protein Synthesizer *}

\author{
Andrew Schoenrock \\ School of Computer Science \\ Carleton University \\ Ottawa, Canada \\ aschoenr@scs.carleton.ca \\ Alex Wong \\ Department of Biology \\ Carleton University \\ Ottawa, Canada \\ alex.wong@carleton.ca
}

\author{
Daniel Burnside \\ Department of Biology \\ Carleton University \\ Ottawa, Canada \\ daniel.burnside@carleton.ca \\ Ashkan Golshani \\ Department of Biology \\ Carleton University \\ Ottawa, Canada \\ ashkan.golshani@carleton.ca
}

\author{
Houman Moteshareie \\ Department of Biology \\ Carleton University \\ Ottawa, Canada \\ houman.moteshareie@carleton.ca \\ Frank Dehne \\ School of Computer Science \\ Carleton University \\ Ottawa, Canada \\ frank@dehne.net
}

\begin{abstract}
Engineered proteins are synthetic novel proteins (not found in nature) that are designed to fulfill a predetermined biological function. Such proteins can be used as molecular markers, inhibitory agents, or drugs. For example, a synthetic protein could bind to a critical protein of a pathogen, thereby inhibiting the function of the target protein and potentially reducing the impact of the pathogen. In this pas per we present the In-Silico Protein Synthesizer (InSiPS), a massively parallel computational tool for the IBM Blue Gene/Q thas is aimed at designing inhibitory proteins. More precisely, InSiPS designs proteins that are predicted to interact with a given target protein (and may inhibit the target's cellular functions) while lesving non-target proteins unaffected (to minimize side-effects). As proof-of-concepts, two InSiPS designed proteins have been synthesized in the lab and their inhibitory properties have been experimentally verified through wet-lab experimentation.
\end{abstract}

\section{Keywords}

Applications, Bioinformatics and Computational Biology, Computational Medicine and Bicengineering, Synthetic Biology, Protein Design

\section{INTRODUCTION}

Protein engineering refers to an expansive field of research that strives to design functionally optimized proteins. The modification of existing proteins to improve or arnend endogenous function [4], and de novo protein design, which generates novel proteins optimized for specific function(s) [17]. Protein engineering can be considered the reverse problern of protein function prediction. In function prediction, one is given a protein serquence and tasked with determining the protein function, for example by approximating its three dimensional structure (protein folding) and determining the protein's interactions with other proteins (protein docking). In protein engineering, we are given a desired function of a yet unknown protein, and the task is to generate a secquence for a protein with such properties.

The function of a protein $A$ is mediated by its interactions with other proteins, ie. set of target proteins with which $A$ intersets and the set of non-target proteins with which $A$ does not interact [14]. Designed proteins with a given set of target and non-target proteins can be used as regulatory proteins that enhance or inhibit the function of their target protein(s). Desagning proteins that can bind a target protein in a regulatory capacity can also socomplish the gosl of modifying the structure of the target protein. Of partic ular interest is the design of inhibitory proteins which bind to a single target protein with high specificity (i.e. avoiding non-target proteins), thereby preventing the target from performing its normal biological function $[1,5]$. For example, a designed inhibitory protein could attach itself to a critical protein of a pathogen, thereby inhibiting the function of that target protein and potentially reducing the impact of 
Phosphatase complex Pph3/Psy2 is involved in regulation of efficient non-homologous end-joining pathway in the yeast Saccharomyces cerevisiae.

Omidi K, Hooshyar M, Jessulat M, Samanfar B, Sanders M, Burnside D, Pitre S, Schoenrock A, Xu J, Babu M, Golshani A.

PLoS One. 2014 Jan 31;9(1):e87248. doi: 10.1371/journal.pone.0087248.

\section{Phosphatase Complex Pph3/Psy2 Is Involved in Regulation of Efficient Non-Homologous End-Joining Pathway in the Yeast Saccharomyces cerevisiae}

\footnotetext{
Katayoun Omidi ${ }^{1,2}$, Mohsen Hooshyar ${ }^{1,2}$, Matthew Jessulat ${ }^{1,3}$, Bahram Samanfar ${ }^{1,2}$, Megan Sanders ${ }^{1,2}$, Daniel Burnside ${ }^{1,2}$, Sylvain Pitre ${ }^{4}$, Andrew Schoenrock ${ }^{4}$, Jianhua X ${ }^{5}$, Mohan Babu ${ }^{3}$, Ashkan Golshani ${ }^{1,2 *}$

1 Department of Biology, Carleton University, Ottawa, Ontario, Canada, 2 Ottawa institute of Systems Biology, Carleton University, Ottawa, Ontario, Canada, 3 Department of Biochemistry, Research and Innovation Centre, University of Regina, Regina, Saskatchewan, Canada, 4 Department of Computer Science, Carleton University, Ottawa, Ontario, Canada, $\mathbf{5}$ College of Pharmaceutical Sciences, Zhejian University, Hangzhou, Zhejiang, China
}

\section{Abstract}

One of the main mechanisms for double stranded DNA break (DSB) repair is through the non-homologous end-joining (NHE) pathway. Using plasmid and chromosomal repair assays, we showed that deletion mutant strains for interacting proteins Pph3p and Psy2p had reduced efficiencies in NHE. We further observed that this activity of Pph3p and Psy2p appeared linked to cell cycle Rad53p and Chk1p checkpoint proteins. Pph3/Psy2 is a phosphatase complex, which regulates recovery from the Rad53p DNA damage checkpoint. Overexpression of Chk1p checkpoint protein in a parallel pathway to Rad53p compensated for the deletion of PPH3 or PSY2 in a chromosomal repair assay. Double mutant strains $4 p p h 3 / 4 c h k 1$ and $4 p$ sy2/4chk1 showed additional reductions in the efficiency of plasmid repair, compared to both single deletions which is in agreement with the activity of Pph3p and Psy2p in a parallel pathway to Chk1p. Genetic interaction analyses also supported a role for Pph3p and Psy2p in DNA damage repair, the NHEJ pathway, as well as cell cycle progression. Collectively, we report that the activity of Pph3p and Psy2p further connects NHEJ repair to cell cycle progression. 


\section{Spindle Checkpoint Factors Bub1 and Bub2 Promote DNA Double-Strand Break Repair by Nonhomologous End Joining.}

Jessulat M, Malty RH, Nguyen-Tran DH, Deineko V, Aoki H, Vlasblom J, Omidi K, Jin K, Minic Z, Hooshyar M, Burnside D, Samanfar B, Phanse S, Freywald T, Prasad B, Zhang Z, Vizeacoumar F, Krogan NJ, Freywald A, Golshani A, Babu M.

Mol Cell Biol. 2015 Jul;35(14):2448-63. doi: 10.1128/MCB.00007-15.

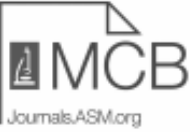

\section{Spindle Checkpoint Factors Bub1 and Bub2 Promote DNA Double- Strand Break Repair by Nonhomologous End Joining}

\footnotetext{
Matthew Jessulat, ${ }^{\mathrm{a}, \mathrm{b}}$ Ramy H. Malty, ${ }^{\mathrm{a}}$ Diem-Hang Nguyen-Tran, ${ }^{\mathrm{a}}$ Viktor Deineko, ${ }^{\mathrm{a}}$ Hiroyuki Aoki, James Vlasblom, ${ }^{\mathrm{a}}$ Katayoun Omidi, Ke Jin, ${ }^{\mathrm{a}, \mathrm{c}}$ Zoran Minic, ${ }^{\mathrm{a}}$ Mohsen Hooshyar, ${ }^{\mathrm{b}}$ Daniel Burnside, ${ }^{\mathrm{b}}$ Bahram Samanfar, ${ }^{\mathrm{b}}$ Sadhna Phanse, ${ }^{\mathrm{a}}$ Tanya Freywald, ${ }^{\mathrm{d}}$ Bhanu Prasad, Zhaolei Zhang, ${ }^{\circ}$ Franco Vizeacoumar, ${ }^{\text {d }}$ Nevan J. Krogan, ${ }^{,}$Andrew Freywald, ${ }^{d}$ Ashkan Golshani, ${ }^{b}$ Mohan Babu ${ }^{\mathrm{a}}$

Department of Biochemistry, Research and Innovation Centre, University of Regina, Regina, Saskatchewan, Canada"; Department of Biology and Ottawa Institute of Systems Biology, Carleton University, Ottawa, Ontario, Canadab; Banting and Best Department of Medical Research, Donnelly Centre, University of Toronto, Toronto, Ontario, Canadac; Cancer Research Unit, Saskatchewan Cancer Agency, Saskatoon, Saskatchewan, Canadad; Department of Medicine, Regina Qu'Appelle Health Region, Regina, Saskatchewan, Canada"; Department of Cellular and Molecular Pharmacology, University of California, San Francisco, San Francisco, California, USA'

The nonhomologous end-joining (NHEJ) pathway is essential for the preservation of genome integrity, as it efficiently repairs DNA double-strand breaks (DSBs). Previous biochemical and genetic investigations have indicated that, despite the importance of this pathway, the entire complement of genes regulating NHEJ remains unknown. To address this, we employed a plasmidbased NHEJ DNA repair screen in budding yeast (Saccharomyces cerevisiae) using 369 putative nonessential DNA repair-related components as queries. Among the newly identified genes associated with NHEJ deficiency upon disruption are two spindle assembly checkpoint kinases, Bubl and Bub2. Both observation of resulting phenotypes and chromatin immunoprecipitation demonstrated that Bub1 and -2, either alone or in combination with cell cycle regulators, are recruited near the DSB, where phosphorylated Rad53 or H2A accumulates. Large-scale proteomic analysis of Bub kinases phosphorylated in response to DNA damage identified previously unknown kinase substrates on Tell S/T-Q sites. Moreover, Bubl NHEJ function appears to be conserved in mammalian cells. 53BP1, which influences DSB repair by NHEJ, colocalizes with human BUB1 and is recruited to the break sites. Thus, while Bub is not a core component of NHEJ machinery, our data support its dual role in mitotic exit and promotion of NHEJ repair in yeast and mammals.
} 


\section{Heavy metal sensitivities of gene deletion strains for ITT1 and RPS1A connect their activities to the expression of URE2, a key gene involved in metal detoxification in yeast.}

Moteshareie H, Hajikarimlou M, Mulet Indrayanti A, Burnside D, Paula Dias A, Lettl C, Ahmed D, Omidi K, Kazmirchuk T, Puchacz N, Zare N, Takallou S, Naing T, Hernández RB, Willmore WG, Babu M, McKay B, Samanfar B, Holcik M, Golshani A.

PLoS One. 2018 Sep 19;13(9):e0198704. doi: 10.1371/journal.pone.0198704

\section{PLOS | ONE}

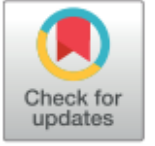

Gopenaccess

Citation: Moteshareie H, Hajikarimlou M, Mulet Indrayanti A, Burnside D, Paula Dias A, Lettl C, et al. (2018) Heavy metal sensitivities of gene deletion strains for $/ T T 1$ and RPS1A connect their activities to the expression of URE2, a key gene involved in metal detoxification in yeast. PLOS ONE 13(9): e0198704. https://doi.ora/10.1371/iournal. pone. 0198704
RESEARCH ARTICLE

Heavy metal sensitivities of gene deletion strains for ITT1 and RPS1A connect their activities to the expression of URE2, a key gene involved in metal detoxification in yeast

Houman Moteshareie ${ }^{1,2}$, Maryam Hajikarimlou $\odot^{1}$, Alex Mulet Indrayanti' ${ }^{2}$, Daniel Burnside ${ }^{1}$, Ana Paula Dias ${ }^{1}{ }^{1}$, Clara Lettl ${ }^{1}$, Duale Ahmed ${ }^{1,3}$, Katayoun Omidi ${ }^{1}$ Tom Kazmirchuk ${ }^{1}$, Nathalie Puchacz ${ }^{1}$, Narges Zare ${ }^{1}$, Sarah Takallou', Thet Naing ${ }^{3}$, Raúl Bonne Hernández $z^{1,4}$, William G. Willmore ${ }^{2}$, Mohan Babu ${ }^{5}$, Bruce McKay ${ }_{\odot}{ }^{1}$, Bahram Samanfar ${ }^{1,6}$, Martin Holcik $\odot^{3}$, Ashkan Golshani $\odot^{1,2 *}$

1 Department of Biology and Ottawa Institute of Systems Biology, Carleton University, Ottawa, Ontario, Canada, 2 Institute of Biochemistry, Carleton University, Ottawa, Ontario, Canada, 3 Department of Health Sciences, Carleton University, Ottawa, Ontario, Canada, 4 University Federal de Săo Paulo Departamento de Química, Laboratorio de Bioinorgânica e Toxicologia Ambiental - LABITA, Săo Paulo, Brazil,

5 Department of Biochemistry, Research and Innovation Centre, University of Regina, Regina, Canada, 6 Agriculture and Agri-Food Canada, Ottawa Research and Development Centre (ORDC), Ottawa, Ontario, Canada

\section{* Ashkan.Golshani@ carleton.ca}

\section{Abstract}

Heavy metal and metalloid contaminations are among the most concerning types of pollutant in the environment. Consequently, it is important to investigate the molecular mechanisms of cellular responses and detoxification pathways for these compounds in living organisms. To date, a number of genes have been linked to the detoxification process. The expression of these qenes can be controlled at both transcriptional and translational levels. 
Global landscape of cell envelope protein complexes in Escherichia coli.

Babu M, Bundalovic-Torma C, Calmettes C, Phanse S, Zhang Q, Jiang Y, Minic Z, Kim S, Mehla J, Gagarinova A, Rodionova I, Kumar A, Guo H, Kagan O, Pogoutse O, Aoki H, Deineko V, Caufield JH, Holtzapple E, Zhang Z, Vastermark A, Pandya Y, Lai CC, EI Bakkouri M, Hooda Y, Shah M, Burnside D, Hooshyar M, Vlasblom J, Rajagopala SV, Golshani A, Wuchty S, F Greenblatt J, Saier M, Uetz P, F Moraes T, Parkinson J, Emili A. Nature Biotechnology. 2018 Jan;36(1):103-112. doi: 10.1038/nbt.4024.

\section{nature biotechnology}

Resource | Published: 27 Novem ber 2017

\section{Global landscape of cell envelope protein complexes in Escherichia coli}

Mohan Babu ${ }^{4}$, Cedoljub Bundalovic-Torma [...] Andrew Emili

Nature Biotechnology 36, 103-112(2018) | Download Citation $\underline{\downarrow}$ 